\title{
Recoil-Ion and Electron Momentum Spectroscopy: Reaction-Microscopes
}

\author{
J. Ullrich ${ }^{1}$, R. Moshammer ${ }^{1}$, A. Dorn ${ }^{1}$, R. Dörner ${ }^{2}$, L. Ph. H. Schmidt ${ }^{2}$, H. Schmidt-Böcking ${ }^{2}$ \\ ${ }^{1}$ Max-Planck-Institut für Kernphysik, Saupfercheckweg 1, D-67119 Heidelberg, Germany \\ ${ }^{2}$ Institut für Kernphysik, Universität Frankfurt, August Euler Str. 6, D-60486, Germany
}

\begin{abstract}
Recoil-ion and electron momentum spectroscopy is a rapidly developing technique that allows one to measure the vector momenta of several ions and electrons resulting from atomic or molecular fragmentation. In a unique combination, large solid angles close to $4 \pi$ and superior momentum resolutions around a few percent of an atomic unit (a.u.) are typically reached in state-of-the art machines, so-called Reaction Microscopes. Evolving from recoilion and COLd Target Recoil-Ion Momentum Spectroscopy" (COLTRIMS), Reaction Microccopes - the "bubble chambers of atomic physics" - mark the decisive step forward to investigate many-particle quantum-dynamics occurring when atomic and molecular systems or even surfaces and solids are exposed to time-dependent external electromagnetic fields.

The present review concentrates on just these latest technical developments and on at least four new classes of fragmentation experiments that have emerged within about the last five years. First, multi-dimensional images in momentum space brought unprecedented information on the dynamics of single-photon induced fragmentation of fixed-in-space molecules and on their structure. Second, a break-through in the investigation of highintensity short-pulse laser induced fragmentation of atoms and molecules has been achieved by using Reaction Microccopes. Third, for electron and ion-impact, the investigation of twoelectron reactions has matured to a state such that first fully differential cross sections (FDCS) are reported. Forth, comprehensive sets of FDCS for single ionisation of atoms by ion-impact, the most basic atomic fragmentation reaction, brought new insight, a couple of surprises and unexpected challenges to theory at $\mathrm{keV}$ to $\mathrm{GeV}$ collision energies. In addition, a brief summary on the kinematics is provided at the beginning. Finally, the rich future potential of the method is shortly envisaged.
\end{abstract}




\section{Introduction}

\section{Kinematics: A Brief Summary}

2.1 General Considerations

2.2 Fast Ion-Atom Collisions

2.3 Collisions with Photons

\section{Imaging Techniques}

3.1 Reaction Microscopes

3.1.1 Imaging of Ions

3.1.2 Imaging of Electrons

3.2 Target-Preparation

3.2.1 Supersonic Gas-Jets

3.2.2 Magneto-Optical-Traps (MOTRIMS)

3.3 New Developments

\section{Results}

4.1 Photons

4.1.1 Double Ionisation of Atoms: A Brief Summary

4.1.2 Photo Ionisation of Fixed-in-Space Molecules

4.2 Single and Multiple Ionisation in Intense Laser Fields

4.2.1 Single Ionisation: Recollision in the Tunnelling Regime

4.2.2 Double Ionisation: Non-Sequential and Sequential

4.2.3 Correlated Motion of the Electrons in the Sequential and Non-Sequential Regimes

4.2.4 Final State Coulomb Repulsion and Sub-Threshold Recollision

4.2.5 Transverse Momentum Exchange

4.3 Electron Impact Ionisation

4.3.1 Single and Double Ionisation

4.3.2 Ionisation plus Excitation

4.3.3 Laser Assisted (e,2e)

4.4 Ion Collisions

4.4.1 Electron Capture: Dynamics and Spectroscopy

4.4.2 Projectile Ionisation: A Novel Approach to (e,2e)-Experiments on Ions?

4.4.3 Ionisation by Slow Projectiles: Saddle Point Electrons

4.4.4 Ionisation by Fast Projectiles: Attosecond Pulses 4.4.4.1 Sinlge ionisation at small and large perturbation 4.4.4.2 Double ionisation at small and large perturbation 4.4.4.3 Multiple ionisation at large perturbation

4.4.5 A Short Summary

\section{A View into the Future}




\section{Introduction}

The present review tries to give an experimentally biased overview on the present state of understanding and research of a tremendously fast developing field, namely the investigation of the quantum-dynamics of fragmenting atoms and molecules. In striking contrast to the profound theoretical knowledge based on very precise experimental data that has been achieved in the investigation of the static structure of atoms and molecules, even the most simple and, thus, fundamental dynamical problems still pose severe challenges to theory:

Only three years ago, it has been reported that single ionisation of the hydrogen atom by electron impact, the most basic fragmentation reaction, has been solved in a mathematically consistent way (Rescigno et al (1999) and references therein, Bray (2002)). The methods employed, a large scale partial wave expansion, made use of massively parallel supercomputers. Until now however, it has not been demonstrated to be practicable for ion encounters or electron impact at lower or even higher energies. More recently, threedimensional imaging of the electron emission for single ionisation of helium by fast bare ionic projectiles brought to light severe discrepancies with existing theoretical descriptions in the perturbative (Schulz et al 2003) as well as in the non-perturbative regime (Moshammer et al 2001). At low collision velocities, where "saddle point electrons" were once predicted to be emitted by Olson $(1983,1986)$, rich structures in the impact parameter dependent electron momentum distributions (Dörner et al 1996; Abdallah et al 1997, 1998; Edgü-Fry E et al 2002; Afaneh et al 2002) are still not quantitatively explained by theory (Macek and Ovchinnikov 1998; Sidky et al 2000, Sidky and Lin 2001).

Until now, the more "complicated" complete disintegration of a helium atom, the simplest many-electron system where correlation has to be taken into account (see e.g. McGuire (1995,1997), Ford and Reading (1988, 1990), Bronk et al (1998)), has been successfully described theoretically on the level of FDCS only for fragmentation by single photons (for a recent review see e.g. Briggs and Schmidt (2000)) or a fast electron impact (Kheifets et al 1999, Dorn et al 2001, 2002, 2002a). Helium double ionisation in the non-perturbative regime induced by intense femtosecond laser fields or by strong, ion-generated attosecond pulses seems to be far from being solved theoretically. Multiple ionisation finally, poses insurmountable problems to quantum theory on the level of fully differential cross sections and available data have to be compared to predictions of classical many-particle calculations (Schulz et al 2000). 
In the recent past, essentially since less than a decade ago, the field was revolutionised from the experimental point of view by the invention of advanced, innovative many-particle momentum imaging and projection techniques based on large area position- and time-sensitive multihit electron and ion detectors. The integration of target preparation, projection techniques and detector development (Martin et al 1981, Sobottka and Williams 1988, Jagutzki et al 1998) lead to todays Reaction Microccopes - the "bubble chambers of atomic and molecular physics" - developed by Moshammer et al $(1994,1996)$ and Ullrich et al (1995). They enable to measure the vector momenta of several fragments (ions, electrons, molecular ions) with unprecedented large solid angles, often reaching hundred percent of $4 \pi$, at extreme precision: Energy resolutions below $1 \mathrm{meV}$ are achieved for slow electrons while ion momenta are routinely recorded at the $1 \mu \mathrm{eV}$ level, corresponding to a temperature of a few milliKelvin (for the detection of low-energy electrons $(<5 \mathrm{eV})$ in coincidence with recoil-ions see also Kravis et al (1996), Dörner et al (1996a,b) and Abdallah et al (1998)). Additional technical progress in the projectile beam preparation, namely the availability of nanosecond pulsed electron or ion beams as well as intense pulsed photon beams from $3^{\text {rd }}$ generation light sources or kilohertz, ultra-fast strong laser systems, accelerated the data-taking efficiency decisively. Now, not only "kinematically complete" measurements have become feasible but moreover, fully differential cross sections can be projected out of huge data sets.

In parallel, despite of general problems, substantial progress has been achieved in the theoretical treatment of fragmenting Coulomb systems, driven by conceptual innovations as well as by the dramatic growth of computational capabilities in recent years. For example, the exterior complex scaling method mentioned above, even if not easily to be generalized, nevertheless did solve the fundamental three-particle Coulomb problem in excellent agreement with experimental results. Moreover, convergent close coupling calculations as well as hyperspherical R-matrix methods combined with semi-classical outgoing waves are nowadays able to reliably predict fully differential fragmentation patterns for photo double ionisation of helium. Meanwhile, within the last three years, the close coupling technique has been successfully applied to describe double ionisation by charged particle impact at high velocities and first successful attempts have been undertaken to implement higher-order contributions at lower energies. In addition, S-matrix approaches to describe the interaction of strong laser fields with atoms, numerical grid methods to directly integrate the Schrödinger equation, hidden crossing techniques for ion impact at low collision energies, time-dependent density func- 
tional theory to approach "true" many-electron problems and many more were successfully developed or applied in the recent past (see e.g. Ullrich and Shevelko (2003)).

Historically, Reaction Microccopes have emerged from "Recoil-Ion Momentum Spectroscopy" (RIMS) and COLTRIMS, continuously developed since the first recoil-ion momentum measurements by Ullrich and Schmidt-Böcking in Frankfurt (Ullrich 1987, Ullrich and Schmidt-Böcking 1987, Ullrich et al 1988, 1988a). Few groups world wide, the one at Kansas State University with Cocke and Ali, of Grandin and Cassimi at the GANIL in Caen, the group at the University of Frankfurt with Schmidt-Böcking, Dörner, Mergel, Schmidt, Jagutzki and others, those of Ullrich, Moshammer and Dorn at GSI, Freiburg University and now at the Max-Planck-Institute in Heidelberg made decisive contributions over 15 years to arrive at the present state of sophistication. The historical development as well as the wealth of results obtained with RIMS, the earlier recoil-ion momentum spectrometers and COLTRIMS, the high-resolution $4 \pi$-detection of the recoil ion alone (sometimes COLTRIMS is also used as a synonym for simultaneous ion and electron momentum spectroscopy) were summarized in detail in several previous reviews on the field (Ullrich 1994, Ullrich et al 1997, Dörner et al 2000).

Therefore, and in the light of explosion-like progress within the last five years, the present review exclusively reports on the most recent experimental results and technical developments which are not or rarely covered in the previous reviews. After a brief summary of the kinematics in Chapter 2, the latest technical developments are described in Chapter 3. In Chapter 4 single photon, intense laser, electron and fast ion impact induced fragmentation processes are reported within four Sections. Compared to early work with single photons at synchrotrons using Reaction Microccopes, research has been strongly evolving towards molecular physics, exploring the fragmentation dynamics of fixed-in-space molecules which is described in Section 4.1. Since the last review we have witnessed the first successful recoilion momentum measurement on intense laser induced break-up reactions of atoms performed by Moshammer et al (2000) and Weber et al (2000). Due to the rapid development in laser technology producing shorter and shorter pulses down to two optical cycles, achieving phase stabilization within the pulse envelope, producing attosecond higher harmonic photon pulses etc., this topic progresses extremely fast. Reaction Microccopes start to play a key-role in the field and the whole Section 4.2 is devoted to it. Furthermore, electron impact induced twoelectron processes "just under way" as reported by Dörner et al (2000) have seen a breakthrough since then with a set of kinematically complete $(\mathrm{e}, 3 \mathrm{e})$ or ionisation-plus-excitation measurements and first successful attempts to investigate laser-assisted (e,2e) reactions, de- 
scribed in Section 4.3. Finally as reviewed in Section 4.4, even in the traditional field of recoil-ion momentum spectroscopy, i.e. in ion-atom collision physics, tremendous progress has been achieved within the last five years: Three-dimensional imaging of the electron emission in single ionisation at very low (quasi-molecular regime) and quite large velocities (perturbative regime) as well as for fast highly-charged ion impact (non-perturbative regime) revealed a couple of surprises testing theory in regimes that are not accessible for electron impact. Electron capture measurements have been performed in a storage ring for the first time, identifying higher-order Thomas mechanisms (Schmidt et al 2002), as well as at an Electron Beam ion Trap (EBIT) obtaining spectroscopic resolution for highly excited ionic states. A fully differential measurement for He double ionisation was reported and compared to (e,3e) results and projectile ionisation was explored for the first time in a kinematically complete experiment. Finally, in Chapter 5 we will shortly envisage the rich future for the physics to be explored with Reaction Microccopes.

\section{Kinematics: A Brief Summary}

In this Chapter a short summary on the non-relativistic kinematics of atomic fragmentation processes, induced by electron, ion, photon or laser-pulse impact will be provided with some emphasis given to the role of the recoiling target ion and the information which is contained in its recoil-momentum. The main purpose is, to help the reader understanding the results presented in Chapter 4 without having to search for equations in other articles. Derivations of the equations, relativistic kinematics, and a wealth of examples as well as molecular fragmentation kinematics can be found in the former reviews on the topic (Ullrich et al 1994, Ullrich et al 1997, Dörner et al 2000) and in a recent book (Ullrich and Shevelko 2003). Throughout this review atomic units (a.u.) are used, where the electron mass $m_{e}$ and charge $e$ as well as Planck's constant $\hbar=h / 2 \pi$ are set to unity $\left(m_{e} \equiv e \equiv \hbar \equiv 1\right)$ and the velocity of light $c=137$.

\subsection{General Considerations}

The general scheme of an impact induced fragmentation reaction is sketched in Figure 1: A projectile with an incoming momentum $\vec{P}_{P}^{i}=\left(P_{P x}^{i}, P_{P y}^{i}, P_{P z}^{i}\right)=\left(0,0, P_{P \|}^{i}\right)$ (laboratory frame) is scattered to a final state $\vec{P}_{P}^{f}=\left(P_{P x}^{f}, P_{P y}^{f}, P_{P z}^{f}\right)$ (for photon absorption $\vec{P}_{P}^{f}=0$ ) transferring a momentum $\vec{q}=-\Delta \vec{P}_{P}=\vec{P}_{P}^{i}-\vec{P}_{P}^{f}$ to the target, where $\Delta \vec{P}_{P}$ is the momentum change of the 
projectile. All charged particle induced reactions are symmetric with respect the projectile propagation direction along the z-axis (azimuthal symmetry) making it convenient to introduce the transverse and longitudinal momenta $\left(P_{\perp}, P_{\|}\right)=\left(\sqrt{\left(P_{x}+P_{y}\right)^{2}}, P_{z}\right)$ as well as the momentum transfer $\vec{q}=\left(q_{\perp}, q_{\|}\right)=\left(q_{\perp}, \Delta E_{p} / v_{p}\right)$, respectively $\left(\Delta E_{p}, v_{p}\right.$ : energy change and velocity of the projectile; $v_{p}=c$ for photon impact).

A possible result of the energy and momentum transfer is the fragmentation of the target atom into $n^{T}$ electrons with momenta $\vec{P}_{e i}^{f}$ ( $n^{T}$-fold ionisation) and one recoiling (excited) target ion with momentum $\vec{P}_{R}^{f}=\left(P_{R x}^{f}, P_{R y}^{f}, P_{R z}^{f}\right)=\left(P_{R \perp}^{f}, P_{R \|}^{f}\right)$. As in the Figure, the index "fo" for "final" is often omitted later on for the target fragment momenta, since we have no measurable initial ones that would have to be distinguished. The total momentum of the target in the initial state $\vec{P}_{R}^{i}+\sum \vec{P}_{e i}^{i}=\vec{P}_{\text {atom }}^{i}$ is zero or has a well-defined value in the ideal case and cooling techniques are applied (see Chapter 3) to approach this limit. For ion impact as is sketched in Figure 2, one often defines an impact parameter $b$ and, in addition, the transfer of $n^{C}$ electrons from the target to the projectile (electron capture) as well as projectile ionisation (electron loss) becomes possible, where $n^{P}$ electrons are released from an ionic projectile. Certainly, all these reaction channels might occur simultaneously within the same collision. As a result, there are $N$ fragments in the final state with $N=n^{T}+1$ for photon, $N=n^{T}+2$ for electron and $N=n^{T}+n^{P}+2$ for ion impact and a total of $n=n^{T}+n^{P}$ electrons released to the continuum. Due to momentum and energy conservation

$$
\Delta E_{p}=E_{P}^{i}-E_{P}^{f}=Q+E_{R}^{f}+\sum_{i=1}^{n} E_{e i}^{f}
$$

the collision kinematics is fully determined if $3 N-3$ linear independent (scalar) momentum components are measured in kinematically complete experiments (neglecting the spin). Then, fully differential cross sections (FDCS) can be extracted. In equation (1), $\Delta E_{p}$ is the energy change of the projectile, $E_{R}^{f}$ and $E_{e i}^{f}$ are the final energies of the recoiling target ion and of the $\mathrm{i}^{\text {th }}$ emitted electron, respectively and $Q=\sum E_{e i_{-} b}^{f}-E_{e i_{-} b}^{i}$ is the inelasticity of the reaction, i.e. the change in the total internal energies of the projectile and of the target $\left(E_{e i_{-} b}^{i, f}<0\right.$ : binding energy of the $i^{\text {th }}$ electron in the initial and final state, respectively).

Whereas the kinematical equations usually cannot be further simplified for electron induced reactions, considerable approximations can be made for fast ion collisions as well as for pho- 
ton absorption and Compton scattering, such that physical insight is directly obtained from the recoil-ion or electron momentum distributions, as will be illustrated below. 


\subsection{Fast Ion-Atom Collisions}

For the overwhelming part of all atomic reactions in ion-atom collisions only little momentum, energy and mass compared to the initial momentum $\left(\vec{P}_{P}^{i}\right)$, energy $\left(E_{P}^{i}\right)$ and mass $\left(M_{P}^{i}\right)$ of the incoming projectile is transferred during the encounter. This is true even for small projectile masses (protons and in many cases even for electrons) as well as for comparably violent collisions, where the target atom is multiply ionised in an encounter with a highly charged ion. Under these conditions the longitudinal and transverse momentum balances are decoupled, contain different information on the collision and can be calculated separately on the basis of energy and momentum conservation.

Since the recoil-ion momentum is always measured in the here-described experiments and since it contains a wealth of information, yielding physical insight even for many-electron processes if the electrons are not detected, momentum balances are traditionally expressed within the recoil-ion perspective. Transversally one obtains (non-relativistic):

$$
P_{R \perp}^{f}=-\left(P_{P \perp}^{f}+\sum_{i=1}^{n} P_{e i \perp}^{f}\right)=q_{\perp}-\sum_{i=1}^{n} P_{e i \perp}^{f} .
$$

For pure electron capture (see e.g. Ali et al (1992), Mergel et al (1995), Fischer et al (2002)) or for reactions where $\sum_{i=1}^{n} P_{e i \perp}^{f}$ is small compared to the heavy particle momenta (i.e. at small $b$ or $\left.E_{P}^{i}\right) P_{R \perp}^{f} \approx-P_{P \perp}^{f}$ and the recoil-ion momentum measurement alone yields direct information on the projectile deflection $\vartheta_{P} \approx P_{P \perp}^{f} / P_{P}^{i}$ (see e.g. Ullrich et al (1988a, 1989), Gensmantel et al (1992), Dörner et al (1996a)). If Reaction Microccopes are used, the projectile scattering can be investigated in ionisation or transfer ionisation reactions as well, obtaining detailed information on the many-particle transverse momentum balance. Unprecedented micro or even nano-radian $\vartheta_{P}$-resolution is achieved (see e.g. Moshammer et al (1994, 1996a, 2001), Fischer et al (2003b)).

Expressing the total longitudinal recoil-ion momentum in terms of three different contributions, namely electron capture, loss and ionisation one obtains:

$$
\begin{aligned}
& P_{R \|}^{f}=P_{R \|}^{c a p}+P_{R \|}^{\text {loss }}+P_{R \|}^{i o n} \quad \text { with } \\
& P_{R \|}^{c a p}=\frac{Q}{v_{P}}-n^{C} \cdot \frac{v_{P}}{2} \\
& P_{R \|}^{\text {loss }}=\frac{Q}{v_{P}}+\sum_{i=1}^{n^{P}} \frac{\left(E_{e i}^{f}\right)_{P F}}{v_{P}}
\end{aligned}
$$




$$
P_{R \|}^{i o n}=\frac{Q}{v_{P}}+-\sum_{i=1}^{n^{T}} P_{e i \|}^{f}+\sum_{i=1}^{n^{T}} \frac{E_{e i}^{f}}{v_{P}} .
$$

Energies of electrons released from the projectile (5) are measured in the projectile frame (PF). Thus, apart from the many-particle momentum balance along the beam propagation, the longitudinal recoil-ion momentum contains information on the inelasticity of the reaction. Recently, this has been exploited to obtain structural information about the binding energies of high-lying singly or doubly excited electronic sates by studying single electron capture from $\mathrm{He}$ in collisions with $\mathrm{Ne}^{7+}$ at $v_{P}=0.355$ a.u. reaching a few meV energy resolution as illustrated in Figure 3 (Fischer et al (2002) and references therein for earlier measurements).

\subsection{Collisions with Photons}

A photon with the energy $E_{\gamma}$, absorbed by an atom essentially deposits all its energy into the target electron shell. If the photon momentum $P_{\gamma}=E_{\gamma} / c$ is small compared to the momenta of the atomic fragments, which is true as long as the total inelastic energy transfer is small compared to the sum-energy of emitted photo-electrons, then one obtains for single ionisation:

$$
\begin{aligned}
& \left|\vec{P}_{R}^{f}\right|^{2}=\left(P_{R x}^{f}\right)^{2}+\left(P_{R y}^{f}\right)^{2}+\left(P_{R z}^{f}\right)^{2}=R \\
& \left|\vec{P}_{e}^{f}\right|^{2}=\left(P_{e x}^{f}\right)^{2}+\left(P_{e y}^{f}\right)^{2}+\left(P_{e z}^{f}\right)^{2}=R .
\end{aligned}
$$

In this approximation, electron and recoil-ion emerge into opposite directions compensating their momenta with their momentum vectors ending on a sphere with radius

$$
R=2 \frac{m_{e} M_{R}}{m_{e}+m_{R}}\left(E_{\gamma}-Q\right) .
$$

The recoil-ion energy is $m_{e} / M_{R}$ times smaller than the electron energy and, thus, negligible in most cases. Taking the photon momentum into account results in a shift of the centre of the sphere by $P_{\gamma}=E_{\gamma} / c$ along the photon propagation direction for the recoil ion and of $\left(m_{e} / M_{R}\right) \cdot\left(E_{\gamma} / c\right)$ for the electron, respectively. Moreover, the radii of the spheres shrink.

In Figure 4, momentum distributions in the (x,y)-plane are shown for single ionisation of helium by the absorption of one photon (a), for multi-photon above-threshold ionisation of argon (b), for tunnelling ionisation of $\mathrm{Ne}$ in an intense photon pulse (c) and for Compton scattering (d). The circular shape is clearly visible in the recoil-ion momentum distribution for the absorption of an $80 \mathrm{eV}$ photon by He (Figure 4a), with different inner circles due to the simul- 
taneous excitation of the remaining $\mathrm{He}^{+}$electron to various n-levels. For the outer circle where the $\mathrm{He}^{+}$ions are in their ground state, one observes a clean dipolar intensity distribution with its maximum along the horizontal polarization direction. For multi-photon absorption (Figure 4b) several rings are found in the electron momentum distribution, separated by the recoil-momentum of one individual laser photon. Close to zero momentum the circular structure is washed out due to the distortion of the atomic levels as well as of the continuum by the strong laser field. Moreover, only small parts of the circles are actually populated with a distinct maximum along the horizontal polarization direction and a rapid decrease towards larger angles, indicating that considerable momentum can be transferred if few photons are involved. If the field becomes very strong $\left(10^{15} \mathrm{~W} / \mathrm{cm}^{2}\right)$, the circular structure disappears completely (Figure 4c). Now, the recoil-ion momentum pattern is strongly extended along the polarization axis. The distribution obtained along the $\mathrm{x}$ - and $\mathrm{y}$-directions can be well understood within a classical field perspective in terms of tunnelling of the active electron through the barrier formed by the modification of the atomic potential in the oscillating laser field (for details see Section 4.2).

For Compton scattering finally, which dominates the photo absorption cross section at large $E_{\gamma}$, the recoil-ion essentially acts as a spectator (inner peak in Figure 4d). Energy and momentum conservation is fulfilled by the photon and the electron alone, leaving the ion just with the momentum of the removed electron, i.e. with the bound-state momentum distribution, namely the „Compton profile“ (see e.g. Samson et al (1994), Spielberger et al (1995,1996), Krässig et al (1999)).

\section{Imaging Techniques}

The rapid and still ongoing development of recoil-ion momentum spectroscopy during the last ten years can undoubtedly be viewed as an experimental break-through for the investigation of any kind of atomic reactions. Whenever atoms or simple molecules interact with electrons, ions or photons and charged target fragments emerge the concept of high resolution recoil-ion measurements resulted in additional and complementary information compared to the traditional methods. State of the art high resolution recoil-ion momentum spectrometers evolved through numerous technical developments like e.g. the implementation of cold supersonic gas-jet targets, the use of well defined electric extraction fields for recoil ions as well as for electrons and the rapid progress in charged particle detection techniques. Among them the use of supersonic jets to produce well localized and internally cold targets (COLd Target Recoil Ion Momentum Spectroscopy, COLTRIMS) can be viewed as the most important ingredient. 
They allowed recoil-ion momentum resolutions far below 1a.u. (atomic unit) which would be impossible with room temperature targets (the momentum spread of room temperature helium is about 3.7 a.u.). Another decisive development was the invention of completely novel and extremely efficient electron imaging concepts. In combination with COLTRIMS these so called "Reaction-Microscopes" enabled the projection of recoil ions and electrons in coincidence and opened up a whole area for kinematically complete atomic reaction studies. Combined with advanced multi-particle detection techniques kinematically complete studies of atomic collision reactions with up to five outgoing particles became feasible (triple ionisation of $\mathrm{Ne}$ in collisions with heavy ions, Schulz et al 2000). Here, we briefly discuss the working principle of these modern spectrometers leaving out a detailed historical overview (for this the reader is referred to Cocke et al (1991), Ullrich et al (1994, 1997), Dörner et al (2000)).

\subsection{Reaction-Microscopes}

The general design of a Reaction-Microscope is shown schematically in Figure 5. A well collimated beam of cold atoms or molecules, usually provided by a cooled supersonic gas jet, is crossed with a projectile beam of any kind. The cross-over defines the effective target volume which is placed in a region where a weak electrostatic field is applied for extraction of charged target fragments. On either side of this acceleration region a field free drift path and a large area position sensitive detector is located to register the recoiling target ions and electrons, respectively. The drift path lengths should be adapted to the acceleration lengths to avoid that different starting positions (potentials) in the acceleration field result in different flight times (time-focusing condition). Electrons and ions are detected after post acceleration by channel plate detectors equipped with structured anodes for position decoding.

From most atomic reactions the recoil ions emerge with kinetic energies of some $\mathrm{meV}$ or below and extraction fields of a few $\mathrm{V} / \mathrm{cm}$ are generally sufficiently high to achieve a $4 \pi$ collection efficiency (with typical spectrometer sizes). However, emitted electrons have usually considerably larger kinetic energies making it much harder to collect them. Most of them simply miss the detector. In order to achieve a high acceptance together with a good resolution for both, electrons and recoil ions in coincidence, a weak homogenous magnetic field (generated by a pair of large Helmholtz coils) is superimposed along the spectrometer axis effectively confining the electron motion in space. Then, with basically two parameters, the magnetic field strength and the extraction voltage, the resolution and the acceptance becomes adjustable over a large range in the electron and ion branch individually. It is essentially this 
zooming option which makes Reaction-Microscopes extremely versatile and which considerably extended the range of applications for coincident electron-ion imaging.

\subsubsection{Imaging of Ions}

Recoil ions produced in the target zone are accelerated by the electric extraction field onto the position sensitive detector. Then, from the obtained position and time-of-flight (TOF) information of each detected ion, the trajectory can be reconstructed and the initial momentum vector can be calculated unambiguously. The TOF has to be measured with respect to a trigger signal which uniquely defines the time of interaction of a projectile with a single target atom. To do so either a pulsed beam of projectiles (synchrotron-radiation, pulsed lasers, bunched electron or ion-beams) has to be used or single projectiles of a continuous beam have to be detected with a time sensitive detector e.g. after the collision. The TOF spectrum contains two important informations. First, different ion species can be distinguished because they appear as well separated peaks in the TOF spectrum due to the dependence of the flight time on the mass to charge ratio. Second, the shape of these individual peaks contain information about the initial ion momentum. To illustrate this we assume that, for a given spectrometer, ions of a certain mass and charge but with zero initial momentum appear after a flight time $t_{0}$ at the detector. Then, for those ions which emerge with an initial velocity vector pointing toward the detector the measured time of flight will be shorter than $t_{0}$ and vice versa. For a spectrometer with an homogenous extraction field it is possible to assign to each channel in the TOF spectrum the corresponding recoil-ion momentum parallel to the spectrometer axis of $\left(P_{R \|}\right.$ in $a . u ., q U$ in $\mathrm{eV}, a$ in $\mathrm{cm}$ and $t, t_{0}$ in $n s)$

$$
P_{R \|}=8.042 \cdot 10^{-3} \cdot \frac{q U}{a} \cdot\left(t_{o}-t\right)
$$

where $t$ is the actual time of flight and $a$ is the distance over which the ion of charge $q$ is accelerated to an energy of $q U$. This relation is independent on the particle mass and it is valid for ions and electrons as long as their initial kinetic energy is small compared to $q U$. Usually this is very well fulfilled for recoil ions from atomic reactions but not for those resulting from molecular fragmentation. Moreover, only the knowledge about the electric field strength $U / a$ at the source point is required to calculate the ion parallel momentum and no further information about the overall geometry of the spectrometer is needed. Thus, with a time resolution of $1 n s$ in the TOF measurement under otherwise ideal conditions a momen- 
tum resolution below 0.01 a.u. is achievable when a field of $1 \mathrm{~V} / \mathrm{cm}$ is used for extraction. An example of a TOF spectrum is shown in Figure 6 for ionisation of Ar atoms with strong $25 f s$ laser pulses at an intensity of several $10^{13} \mathrm{~W} / \mathrm{cm}^{2}$. The laser polarization direction was chosen to be parallel to the spectrometer axis. The inset of Figure 6 shows the magnified $\mathrm{Ar}^{1+}$ peak with distinct individual peaks corresponding to the absorption of a certain number of $1.5 \mathrm{eV}$ photons above the ionisation threshold. Basically, the recoil ion compensates the momentum of the emitted electron whose kinetic energy is given by the total number of absorbed photons minus the ionisation potential. In this case, structures in the ion momentum distribution (obtained after conversion of the TOF spectrum using (10)) in the order of 0.1 a.u. are clearly resolved. This corresponds to a kinetic energy of the $\mathrm{Ar}^{1+}$ ions of less than $2 \mu \mathrm{eV}$.

The momentum components perpendicular to the spectrometer axis can be determined from the position of impact on the ion detector. For a point like source of ions and for well defined acceleration (homogenous electric field) and ion drift regions the transverse ion momentum vector is given by ( $P_{R \perp}$ in a.u., $m$ in amu, $q U$ in $e V$, distances in $\mathrm{cm}$ )

$$
\vec{P}_{R \perp}=11.6 \cdot \frac{\vec{r}}{(2 a+d)} \cdot \sqrt{q U \cdot m}
$$

where $r$ is the radial displacement on the detector with respect to the position where ions with zero initial momentum hit the detector. The distances $a$ and $d$ denote the acceleration and drift length, respectively. Again, this approximation is very well justified as long as the width $\Delta t$ of the ion TOF-peak is much smaller than the mean flight-time, or in other words, as long as the kinetic energy gain due to the extraction is significantly larger than the initial recoil-ion energy. The achievable momentum resolution depends on the position resolution of the detector and on the extension of the target zone. In many realistic situations the latter is dominating. For a time-focussing spectrometer with an extraction voltage of $10 \mathrm{~V}$ applied over $10 \mathrm{~cm}$ a target extension of $1 \mathrm{~mm}$ causes a transverse momentum uncertainty of $\Delta P_{R \perp}=0.18$ a.u. for He-ions. However, the achievable resolution can be increased significantly either by lowering the extraction voltage or by increasing the spectrometer size as long as the resulting reduction in the transverse momentum acceptance is no matter of concern.

In order to eliminate the influence of the target extension on the transverse momentum resolution spectrometers with so called position-focussing have been developed (see e.g. Dörner et al 1998, Abdallah et al 1998). They focus ions starting at different positions onto a single spot on the detector while the displacement on the detector is still proportional to the initial mo- 
mentum. To achieve position focussing a weak electrostatic lens is implemented into the acceleration region, preferentially as close as possible to the reaction-zone. Thus, the resolution is no longer limited by the source extension but by the imaging properties of the spectrometer. This concept has been used mainly to study electron capture reactions in ion-atom collisions as well as for most photon imact studies where a high resolution is decisive.

\subsubsection{Imaging of Electrons}

The conceptually most obvious scheme for coincident imaging of electrons and ions is to place a second position sensitive detector opposite to the ion detector. In fact, this concept has been used to study low-energy electron emission in ion-atom collisions (Dörner et al 1996a, Edgü-Fry et al 2002) and photoionisation close to threshold (Dörner et al 1996b). For many applications, however, the accepted maximum electron energy in the transverse direction is too small (for early work on the two dimensional imaging of photoelectrons alone, using large extraction fields see Helm et al 1993).

To circumvent this limitation and to increase the electron energy acceptance maintaining at the same time the full resolution in the recoil-ion branch a solenoidal magnetic field is superimposed parallel to the electric field (Moshammer at al 1996). It acts over the whole flight-path and forces electrons onto spiral trajectories from the reaction volume to the detector. Again, the position and TOF information allows an unambiguous reconstruction of the initial electron momentum vector. The longitudinal motion is not changed and the same considerations as those for the recoils can be applied to reconstruct the longitudinal momentum from the electron TOF, however, because of their high kinetic energies, no approximate equations should be used. In the transverse direction (perpendicular to the field axis), the electrons travel along a circle with radius $R=P_{e \perp} /(q B)$ where the time $T$ for one turn is given by the inverse of the cyclotron frequency $\omega=(q B) / m_{e}=2 \pi / T$ ( $B:$ magnetic field strength, $m_{e}$ and $q$ : electron mass and charge, respectively). In useful units these equations are

$$
\omega=9.65 \cdot 10^{-6} \cdot(q B) / m_{e} \quad \text { and } \quad R=12.39 \cdot P_{e \perp} /(q B)
$$

with $\omega$ in $n s^{-1}, q$ and $P_{e \perp}$ in a.u., $B$ in Gauss, $m$ in amu. With a magnetic field of only 10 Gauss all trajectories of electrons with energies up to $100 \mathrm{eV}$ are confined to a cylinder with a radius of $3.3 \mathrm{~cm}$, independent of the electric field strength and independent of the spectrometer geometry. Hence, a detector with $8 \mathrm{~cm}$ diameter is sufficient to achieve a $4 \pi$ collec- 
tion efficiency for electrons with energies of more than $100 \mathrm{eV}$. Under these conditions the revolution time for electrons is $35 \mathrm{~ns}$, i.e. in the range or even smaller than typical electron flight-times. The cyclotron time for ions on the other hand of more than $250 \mu \mathrm{s}$ for He under the above conditions is usually much longer than their flight-times, indicating that the ion trajectories are only weakly affected by the magnetic field. It basically results in a slight rotation of the ion image on the recoil-detector, which is easily compensated by a corresponding back-rotation of the whole ion position distribution. In conclusion, by changing the magnetic field strength the accepted maximum transverse momentum of electrons is adjustable without affecting the recoil-ion imaging.

To calculate the electron transverse momentum vector both informations, position and TOF, are required. To illustrate this, we consider the projection of an electron trajectory onto the electron detector surface, i.e. onto a plane perpendicular to the magnetic field axis (Figure 7). An electron, which is emitted at the origin (point $A$ ) with a transverse momentum $P_{e \perp}$ under a certain angle $\varphi$ with respect to the positive x-axis travels on a spiral trajectory, corresponding to a circle (radius $R$ ) in the transverse plane, before it hits the detector at the point $B$ with a certain displacement $r$ from the center. The radius $R$ is a direct measure of $P_{e \perp}$ (12) while the arc-angle $\omega t$ depends only on the electron TOF. From simple geometrical considerations (Figure 7) one gets

$$
R=\frac{r}{2|\sin (\omega t / 2)|}
$$

Thus, the magnitude of the transverse momentum $P_{e \perp}$ can be calculated from the position of detection, $(r, \varphi)$ in cylindrical coordinates, and the electron TOF $t$ using (12) and (13). With the emission angle $\varphi$, which is given by

$$
\varphi=\vartheta-\omega t / 2
$$

the transverse momentum vector is determined.

What happens if the electron performs more than one complete turn? In this case $\omega t$ has to be replaced by $\omega t-N \cdot 2 \pi$ where $N$ (number of full turns) is the next lowest integer of the ratio $(\omega t) /(2 \pi)$. Still, the assignment of measured quantities and initial momentum is unique, as long as the denominator in (13) is larger than zero. The only prerequisite for reconstruction is the knowledge of the magnetic field strength and direction, respectively. 
Whenever electrons perform exactly $N$ complete turns they hit the detector at the origin (point $A$ in Figure 7) independent of their initial transverse momentum or, in other words, all electrons with flight times equal to a multiple integer of $T$ (the inverse cyclotron frequency) are focussed onto the same spot on the detector and no momentum information is obtained. These specific cases appear as nodal points when the radial displacement on the electron detector is plotted versus the electron time-of-flight (Figure 8). The nodes deliver important and valuable information. First they serve as a good control of the experimental conditions and, more important, they allow a very precise and intrinsic determination of the magnetic field via a measurement of the inverse cyclotron frequency. It is given by the time-distance of two nodes. Whenever important information about the physical process is masked by these nodes, which might happen, the magnetic field can be changed slightly resulting in a corresponding time-shift of the nodal points.

The transverse momentum resolution is given by $\Delta P_{e \perp}=P_{e \perp} \cdot \Delta r / r$, where $\Delta r$ is the effective position uncertainty resulting from both, the detector resolution and the target size. With $\Delta r=1 \mathrm{~mm}$ the transverse momentum resolution is $\Delta P_{e \perp}=0.025 \mathrm{a} . u$. for electrons which hit the detector at $r=4 \mathrm{~cm}$ with $P_{e \perp}=1$ a.u.. This value should be regarded as a lower limit because a finite time resolution, which enters into the determination of the sin-term in (13), as well as magnetic field distortions contribute in addition.

\subsection{Target-Preparation}

A prerequisite for high resolution momentum spectroscopy is a cold atomic or molecular gastarget because typical recoil-ion momenta are of the order or even smaller than the thermal momentum spread at room temperature. In addition, a localized and small target size is needed for particle imaging. Basically two concepts are applied in COLTRIM spectroscopy and Reaction-Microscopes.

\subsubsection{Supersonic Gas-Jets}

With supersonic gas-jet devices well localized and cold atomic or molecular beams are produced. A gas at high pressure $(1-20 \mathrm{~atm})$ is pressed through a small nozzle $(10-100 \mu \mathrm{m})$ into vacuum and thereby accelerated to supersonic speed at the expense of internal motion resulting in an effective cooling. Starting from room temperature the gas is cooled down to temperatures of a only a few Kelvin or even below (for more detailed information see Miller 
1988). One or several stages with differential pumping are used to handle the enormous gasload and to maintain a good vacuum in the reaction chamber. To extract a geometrically well defined atomic beam one or several small "skimmer" apertures are placed behind the nozzle and between the different pumping stages.

For most applications a dense and narrow atomic beam is required which is then crossed with a projectile beam in the interaction chamber. A small interaction volume, which is given by the overlap between the projectile and target-beam, can be obtained with narrow gas beams resulting in a high momentum resolution for particle imaging. Without too much effort particle densities in order of $10^{11}$ to $10^{12} \mathrm{~cm}^{-3}$ at a distance of $10 \mathrm{~cm}$ away from the nozzle are reachable with supersonic gas-jets.

Though the target gas is cooled by a factor of 1000 , or even more if pre-cooling of the gas is used, the residual momentum spread certainly sets a lower limit in the achievable ion momentum resolution. In the direction parallel to the gas-jet velocity the intrinsic jettemperature dictates the momentum spread. It is about 0.1 a.u. for expansion of $\mathrm{He}$ at room temperature. The perpendicular momentum spread is much smaller, it is determined by the jet velocity and the divergence of the atomic beam.

Over the last five years supersonic gas-jets became the standard devices to produce cold and localized atomic or molecular targets in connection with recoil-ion momentum spectroscopy (COLTRIMS). With this method a large variety of different gases is accessible ranging from helium or other noble gases up to any type of molecular gases. Cooling of the gas before expansion is useful for helium or molecular hydrogen to reduce the internal gas-jet temperature and to reach the optimum momentum resolution but it can lead to the formation of clusters or liquid droplets if heavy gases are used. One should also be aware that the internal momentum spread increases with the mass $\mathrm{m}$ of the gas atoms $\Delta P_{j e t} \propto \sqrt{m}$ even if the same final jet temperature is obtained. Thus, the generation of a gas jet with a low intrinsic momentum spread of $\Delta P_{R}<0.5$ a.u. along the jet-direction is definitely hard to achieve for gases heavier than Argon.

\subsubsection{Magneto Optical Traps (MOTRIMS)}

A significant further reduction of the target temperature is achievable when laser-cooled atoms trapped in a magneto-optical-trap (MOT) are used as a target. In the first pioneering experiment Wolf and Helm (2000) measured recoil-ion energies of photoionised Rb atoms extracted from a MOT with very high resolution. Basically the same method has been used very 
recently (van der Poel et al 2001, Turkstra et al 2001, Flechard et al 2001) to study single electron capture reactions in $\mathrm{keV}$ ion collisions with atoms trapped in a MOT. In these first experiments an unprecedented resolution of $\Delta P_{R}=0.03$ a.u. in the recoil-ion momentum (Flechard et al 2001) has been achieved by taking benefit from the sub-mK intrinsic temperature of the target. Certainly, this value is not the ultimate resolution set by the gas cloud temperature of typically $100 \mu \mathrm{K}$. It would correspond to a momentum resolution of $\Delta P_{R}=0.003$ a.u. for lithium and $\Delta P_{R}=0.01$ a.u. for rubidium. With typical densities in the range of several $10^{10} \mathrm{~cm}^{-3}$ and radii of $1 \mathrm{~mm}$ or below the atomic clouds trapped in MOT's are ideally suited as targets for recoil momentum spectroscopy (MOTRIMS). Another important aspect is that the palette of possible targets is considerably widened. All alkali and earth alkaline atoms are easily trapped in a MOT, but they are hardly produced and cooled in a supersonic gas-jet. These atoms are in some situations of particular interest because they represent single active electron targets and, therefore, have many features in common with atomic hydrogen, the simplest atomic target which is not directly accessible to COLTRIMS because efficient cooling methods are not at hand. Finally, atoms in traps can be easily prepared in excited states and even aligned with respect to the laboratory frame.

\subsection{New Developments}

In this section a few technical developments related to recoil-ion and electron momentum spectroscopy, which are still ongoing and which might play a role in future applications, are listed and briefly discussed. Though this list is quite incomplete it stands for a still continuing evolution of many-particle imaging devices to investigate atomic and molecular reactions.

The limited number of target species accessible to COLTRIMS is, at least to some extend, a constraint. This is mainly because both conditions, high target density and extremely low temperature, are hard to combine if one thinks on targets like highly excited atoms, metastable helium, atomic hydrogen, vibrationally cold molecules or others. Very recently, the Frankfurt group succeeded in producing a supersonic jet of dense and internally cold metastable Heatoms (Jahnke 2003). A high pressure discharge burning between the entrance and exit surface of a $50 \mu \mathrm{m}$ nozzle collisionally excites He while the following adiabatic expansion into the vacuum reduces the internal temperature. After a subsequent Stern-Gerlach magnet the beam can be used for experiments with oriented and cold metastable helium atoms.

If complex targets are used, for example molecules or clusters, the number of particles ejected from a break-up reaction can be quite large. The desire to detect all of them increases the de- 
mands on the detectors concerning their multi-hit capability. Delay-line anodes for position encoding have been developed which fulfill the requirement of almost negligible dead-time (Jagutzki et al 1998; 2002; 2002a,b). On the other hand, with on-going development of integrated electronic circuits concerning speed and compactness, large area pixel anodes with ultra-fast readout of individual pixels are in the realm of possibility. Presently, work is in progress to develop such an anode where one thousand or more pixels are individually read out using highly integrated electronics mounted on-board directly at the anode.

The flexibility of imaging spectrometers can be increased further if the traditional concept of using only static electric and magnetic fields for projection and guiding of charged particles is skipped. Obviously, the application of pulsed or otherwise time-dependent fields can help to adapt the spectrometer performance to specific problems. To study for example molecular fragmentation maintaining at the same time a high resolution for the emitted electrons a pulsed extraction field can be used. The initially small field is ramped up considerably on a sub- $\mu$ s time-scale whenever an electron hits the detector. Because the ions do not travel over a large distance during this short time they experience a large acceleration ensuring $4 \pi$ detection efficiency for them. This method has been successfully applied for the first time to investigate double photoionisation of molecular hydrogen (Dörner et al 1998a). An electric field between two grids placed at an appropriate position in the ion or electron flight-path can serve as a switchable potential barrier. By gating the applied voltage in synchronization with an external time reference unwanted parts of the TOF spectrum, which would overload the detector, can be masked out. This method has been applied to investigate electron transfer reactions in fast proton helium collisions (Schmidt et al 2002). A similar appraoch has been used for high-resolution electron capture measurements. There the recoil-ions have been extracted exactly parallel to the incoming beam direction. A pulsed electric field acting perpendicular to the ion trajectory within the drift path kicked the recoil-ions onto the detector, which was mounted beneath the projectile beam axis (Fischer et al 2002). In general, the possibilities opened up with pulsed electric fields are by far not yet exhausted.

In order to boost the resolution for high-energy electron imaging $(>100 \mathrm{eV})$ Weber et al (2003) succeeded in combining a retarding electric field with the solenoidal magnetic guiding field. Maintaining the $4 \pi$ solid angle, they reached a resolution of $<1 \mathrm{eV}$ at $300 \mathrm{eV}$ electron energy.

Recently, the imaging concept of the Reaction Microscope has been extended to the detection of electrons emitted from surfaces (Hattass et al 2003, patented by Moshammer and Ullrich 1998). For ions emerging from solids, this technique has been developed early by Mosham- 
mer et al 1990 (Matthäus et al (1991, 1993)) and was recently used by Schmidt-Böcking et al 2003 (see also Jalowy et al (2002; 2002a,b; 2003; 2003a)). 


\section{Results}

\subsection{Photons}

Since 1994 Reaction Microscopes have been successfully used to investigate the interaction of single photons with atoms and molecules at synchrotron radiation sources around the world. The main topics addressed in these studies were:

1. Separation of photoabsorption and Compton scattering in single and double ionisation of helium (Spielberger et al 1995; 1996; 1999; Krässig et al 1999) and the precision measurement of the ratio of double to single ionisation of helium by photoabsorption (Dörner et al 1996b).

2. Kinematically complete studies of double ionisation of helium by photoabsorption.

3. The geometry of small photoexcited molecules (Ueda et al 2003; Muramatsu et al 2002).

4. Photo- and Auger-electron angular distributions from small fixed-in-space molecules. We will briefly review the work on topics 2 and 4 in Subsections 4.1.1 and 4.1.2. For topics 1 and 3 we refer the reader to the original literature.

\subsubsection{Double Ionisation of Atoms: A Brief Summary}

Following the pioneering kinematically complete experiment on double photoionisation of helium by Schwarzkopf et al (1993) today experimental data have been reported for energies from 0.1eV (Huetz and Mazeau 2000) to $450 \mathrm{eV}$ above threshold (Knapp et al 2002) for linear as well as circular polarized light. This work together with the impressive theoretical progress in this field has recently been reviewed by Briggs and Schmidt (2000). We highlight here mainly the contributions of recoil-ion momentum spectroscopy to this field.

The three particles in the final state after photo double ionisation are kinematically completely determined by five linear independent momentum components. They can be measured either by detecting two electrons in coincidence or one electron and the recoiling ion momentum. The key advantage of the coincident imaging of ion and electron momenta is the $4 \pi$ solid angle achieved for all energy sharings between the electrons. This coverage of the total final state phase space has many benefits: 
1. An overview can be gained showing which regions of phase space are most important for the process of double ionisation, i.e. which contribute most to the total cross section.

2. The data can be transformed to any set of coordinates. Thus, not only the traditional angles and energies of the two electrons (or momenta $\vec{k}_{1} \equiv \mathbf{k}_{\mathbf{1}}, \vec{k}_{2} \equiv \mathbf{k}_{\mathbf{2}}$ ) can be chosen, but also collective coordinates like Jacobi coordinates $\left(\vec{k}_{+}=\vec{k}_{1}+\vec{k}_{2} \equiv \mathbf{k}_{+}\right.$and $\left.\vec{k}_{-}=\frac{1}{2}\left(\vec{k}_{1}-\vec{k}_{2}\right) \equiv \mathbf{k}_{-}\right)$or hyperspherical coordinates. In such momentum space images the characteristics of the photo double ionisation process become directly visible (Dörner et al 1996b).

3. The data can be reliably normalized on absolute scale (for details see Dörner et al 1998; Bräuning et al 1998), which is very difficult otherwise (Schwarzkopf and Schmidt 1995). Such absolute data provide a particular sensitive test for theory (Pont et al 1996; Lucey et al 1998; Kheifets and Bray 1998).

To discuss the main physical effects and the main features in the cross section, we start from partially differential data providing an overview progressing to fully differential results.

Figure 9 shows the momentum distributions of one of the electrons and of the doubly charged ion at photon energies of $1 \mathrm{eV}, 20 \mathrm{eV}$ and $100 \mathrm{eV}$ above threshold, respectively. The striking difference between the electronic and ionic distributions reflects part of the mechanisms leading to photo double ionisation. The photon acts upon a charge dipole in the atom. This dipole might be thought of as consisting of the positive ion on one pole and either the center of charge of the electron pair or one of the electrons on the other pole. In either case, the first step of the absorption of the photon will imprint the dipolar characteristics of the linear polarized photon on the distribution of the fragments of a charge dipole. The experiments indicate that the momentum distribution of the nucleus shows a memory of this absorption of the photon. At low excess energies this pattern is completely washed out in the electronic momentum distribution by the electron-electron interaction which is indispensable for double ionisation. For $100 \mathrm{eV}$ excess energy (and even more pronounced at 450 eV (Knapp et al 2002) the fast electron also shows a dipolar emission pattern (Figure 9h). It is very illustrative to switch from single electron coordinates $\vec{k}_{1,2}$ to the Jakobi coordinates $\vec{k}_{+,-}$, corresponding to a change to a molecular perspective of the He atom (Feagin and Briggs 1986). This is most useful if the saddle region of the potential surface governs the final state of the reaction, which is expected close to threshold (Wannier 1953). The two electrons then create a two-center saddle 
potential in which the nucleus is located. The coordinate $\vec{k}_{-}$is along the line connecting the two electrons and $-\vec{k}_{+}$is the momentum vector of the ionic core. At $1 \mathrm{eV}$ and $20 \mathrm{eV}$ there is a clear propensity for an escape of the nucleus perpendicular to $\vec{k}_{-}$. This can be understood by a Wannier type analysis, which predicts, that double ionisation near threshold can only be reached if ionic and electron motion are perpendicular, all other geometries lead to single ionisation (Wannier 1953; Huetz et al 1991; Feagin 1995; 1996; Kazanski and Ostrovsky $1995 ; 1993 ; 1994)$. In the molecular picture the electronic separation $\vec{R}$ is interpreted as a molecular axis and the projection $m$ of the total angular momentum on this axis is taken as an approximate quantum number. This propensity for $m=1$ breaks down at $100 \mathrm{eV}$. Without this propensity, the main motivation for analyzing the process in Jacobi coordinates is lost. The evolution of the three-body system is no longer governed by the saddle region of the potential. We now investigate the internal structure of the two-electron continuum wave in $\vec{k}_{1}, \vec{k}_{2}$ coordinates (Figure 10). Neglecting the (small) photon momentum, the vector momenta of the ion and both electrons have to be in one plane. Figure 10(a) shows the electron momentum distribution in this plane for linear polarized light. The data are integrated over all orientations of the polarization axis with respect to this plane; the $\mathrm{x}$-axis is chosen to be the direction of one electron. The structure of the observed momentum distribution is dominated by two physical effects. First the electron-electron repulsion leads to almost no intensity for both electrons in the same half plane. Second, the ${ }^{1} \mathrm{P}^{\mathrm{o}}$ symmetry leads to a node in the square of the wave function at the point $\vec{k}_{1}=-\vec{k}_{2}$ (Huetz et al 1991; Schwarzkopf et al 1993; Maulbetsch and Briggs 1995; Malegat et al 1997). The corresponding data for left and right circular polarized light are shown in Figure $10(\mathrm{~b}, \mathrm{c})$. They show a strong circular dichroism, i.e. a dependence on the chirality of the light. This might be surprising since the helium atom is perfectly spherical symmetric. Berakdar and Klar (1992) first pointed out that for circular dichroism to occur it is sufficient that the direction of light propagation and the momentum vectors of the electrons span a tripod of defined handedness. This is the case if the two electrons and the light direction are non coplanar and the two electrons have unequal energy (see Berakdar et al (1993); Berakdar (1998); Berakdar (1999); Berakdar and Klar (2001) for a detailed discussion and experimental results (Viefhaus et al 1996; Kheifets and Bray 1998; Mergel et al 1998; Kheifets et al 1999a; Soejima et al 1999; Achler et al 2001; Collins et al 2002).

Finally, fully differential cross section can be investigated. The main features seen here at low to moderate excess energies are the interplay between selection rules resulting from the ${ }^{1} \mathrm{P}^{\mathrm{o}}$ 
symmetry and the electron repulsion. A detailed discussion goes beyond the scope of the present review; we refer the reader to (Briggs and Schmidt 2000). In general, very good agreement is found between the experimental data even on absolute scale and the most advanced theoretical approaches (Kheifets and Bray 1998; Kheifets and Bray 1998a; Kheifets and Bray 2000; Malegat et al 2000; Malegat et al 2002; Selles et al 2002; Pont and Shakeshaft 1995; Pont and Shakeshaft 1996a; Colgan et al 2001; Colgan and Pindzola 2002). At very high excess energies of $450 \mathrm{eV}$ finally (Knapp et al 2002), one electron is found to leave fast, carrying away most of the photon energy and angular momentum. In the angular distributions of the second, slow electron clear traces of the mechanism which led to its ejection can be found (see also Teng and Shakeshaft (1994); Keller (2000)). Electrons emitted via the shake-off mechanism are expected to be isotropic or slightly backward directed with respect to the primary electron, while electrons knocked out in a binary collision (TS1-mechanism) will yield $90^{\circ}$ between the two electrons. At $529 \mathrm{eV}$ photon energy the electron angular distributions show a dominance of the shake-off mechanism for secondary electrons which have very low energy $(2 \mathrm{eV})$ and display clear evidence that an inelastic electron-electron scattering is necessary to produce secondary electrons of $30 \mathrm{eV}$ (Knapp et al 2002) (see Figure 11).

\subsubsection{Photoionisation of Fixed-in-Space Molecules}

The combination of photons of narrow bandwidth and well controlled polarization from synchrotron radiation sources with electron and ion imaging provides an extremely versatile and powerful tool for molecular physics. The photon allows complete control of the angular momentum and the total energy of the molecular states populated. The kinematically complete measurement of the emitted electron(s) and fragment ions allows one to measure the response of the molecule to the energy and angular momentum deposition. Such processes of photoexcitation or ionisation of molecules can often be split in two independent sub-processes (Born-Oppenheimer Approximation): First, a fast electronic transition occurs, followed by the slow process of dissociation of the molecular ion. The ion momenta resulting from dissociation are usually in the $\mathrm{eV}$-regime, i.e. at least two orders of magnitude higher than in atomic ionisation processes. Of particular interest are dissociation processes which are fast compared to typical rotation times of the molecule. In this case the molecular orientation at the instant of the electronic transition can be determined a posteriori by measuring the direction of the fragments (axial recoil approximation; Zare (1972)). 
Electron angular distributions for molecules fixed in space by detection of the molecular fragments have been first performed by Shigemasa and coworkers with traditional analyzers for electrons and ions (Shigemasa et al 1995) (see also Shigemasa et al (1998); Watanabe et al (1997); Cherepkov et al (2000); Cherepkov et al (2000a); Ito et al (2000); Ito et al (2000a); Motoki et al (2002) for later experiments by this group). One other group has combined timeof-flight electron spectrometers (Golovin et al 1997; Heiser et al 1997; Gessner et al 2002) with imaging of the molecular fragments.

We will restrict the further discussion on experiments where coincident imaging of electrons and ionic fragments has been used for valence or innershell ionisation. A variety of molecules has been studied, including CO (Landers et al 2001; Weber et al 2001a; Jahnke et al 2002; Hikosaka and Eland 2000), $N_{2}$ (Jahnke et al 2002; Hikosaka and Eland 2000a), NO (Gessner et al 2002), $\mathrm{CO}_{2}$ (Saito et al 2003), $\mathrm{O}_{2}$ (Heiser et al 1997; Lafosse et al 2002; Lafosse et al 2001), $\mathrm{H}_{2}$ (Dörner et al 1998a; Hikosaka and Eland 2002), $\mathrm{CH}_{3} \mathrm{Cl}$ (Hikosaka et al 2001), $\mathrm{CH}_{3} F$ (Hikosaka et al 2001) $\mathrm{C}_{2} \mathrm{H}_{2,4,6}$ (Osipov et al 2003).

From the energy correlation between photoelectrons, Auger electrons and sum of the kinetic energy of the fragments (kinetic energy release KER) mostly the ionisation or excitation channels (Hikosaka and Eland 2002; Lafosse et al 2000; Lafosse et al 2001) or the Auger decay pathes (Weber et al 2003) can be disentangled. One example where $N O$ is ionised by absorption of a $23.64 \mathrm{eV}$ photon is shown in Figure 12. The diagonal lines correspond to different excited final states of the $N^{+}$fragment. The three islands show different reaction pathes which are shown in the correlation diagram in Figure 12b.

For a well controlled reaction pathway the angular distribution of the electrons can be studied. Contrary to the case of atomic photo ionisation, photoelectron angular distributions from molecules show a very rich structure (Dill 1976; Dehmer and Dill 1976). Examples are shown in Figure 13. What are the physical mechanisms producing this structure? From the angular momentum perspective these distributions show that high angular momenta are present in the electronic wave function. The one unit of angular momentum of the photon is absorbed by the molecule creating an entangled state of the continuum electron and the molecular ion. The angular momentum part of the continuum electron and the molecular ion wavefunction are mirror images of each other. The electron leaves the rotational molecular ion wave packet behind which is the coherent superposition of many angular momentum states (Choi et al 1994) and, vice versa, the continuum electron wave function is a superposition of many angular momentum components. This angular momentum exchange between electron and molecular ion results from a multiple scattering of the electron wave on its way out of the molecular 
potential. If one discusses this process not in an angular momentum basis but in a plane wave perspective (corresponding to the asymptotic detected linear momenta) one might say that the many narrow minima and maxima in the angular distribution are the result of multiple interferences. There are many semi-classical pathes on which the electron can get from its origin e.g. at the carbon center to the final detector direction. All these different pathes have to be added coherently. For example, the direct wave from the carbon has to be added to a wave scattered at the oxygen. The phase shift between the different pathes is determined by the details of the molecular potential. As a consequence, the narrow interference minima and maxima are a very sensitive probe of the potentials used. In reality this problem becomes more interesting by the fact that this potential is time dependent since the molecular wave function reacts to the creation of a hole by the photon. For innershell ionisation for example, very good results are obtained by assuming a frozen potential with a charge of $1 / 2 \mathrm{~K}$-hole instead of the full charge.

The angular distributions also depend strongly on the direction of the photon polarization axis with respect to the molecular axis, since this determines the projection of the photon angular momentum onto this axis (see Figure 13 A-E). By combining linearly and circularly polarized light, the amplitudes and phases of the different angular momentum partial waves can be measured (Gessner et al 2002; Jahnke et al 2002; Motoki et al 2002). In addition, the electron wavelength can be scanned by scanning the photon energy. By this technique diffraction pattern of an electron wave launched from a well defined position inside a molecule can be measured, the molecule can be "illuminated from within" (Landers et al 2001). Once the multiple scattering problem is sufficiently understood theoretically one can use the sensitivity of the electron diffraction pattern on the molecular geometry and potential to test not the scattering calculation but the potential itself. In solid state physics at least the nearest neighbor geometry is often explored in this way by XPD ("X-Ray-Photoelectron Diffraction).

One might ask, if for innershell ionisation the two steps of photoelectron emission and the subsequent Auger electron emission can be treated independently (two-step model) (Dill et al 1980; Kuznetsov and Cherepkov 1996; Guillemin et al 2001). The validity of the two-step model has recently been confirmed in a study of the decay channel and molecular frame angular distribution of the Auger electron from Carbon K-shell photoionised $C O$ (Weber et al 2003). The Auger electron angular distribution in the molecular frame is shown in Figure 14, for two different Auger decay channels. A clear fingerprint of the symmetry of the transition is seen in the angular distribution. A $\Pi$ transition leads to emission preferentially perpendicular to the molecular axis while for a $\Sigma$ transition a strongly peaked emission along the mo- 
lecular axis is found. The K-hole is located at the carbon; the Auger electrons, however, show a striking narrow emission in the direction of the neighboring oxygen atom. Such an emission into the direction of the neighboring atom is known from photoelectron diffraction as 'forward focussing' (Poon and Tong 1984). The screened Coulomb potential next to the source of a photoelectron wave can act as a lens which collects a large amount of the electron flux into the forward direction. A close inspection of this pattern (Figure 14c) shows an oscillatory structure. This is due to a diffraction of the Auger electron wave in the two-center potential as has been reported in the theoretical study of Zähringer et al (1992).

In the field of photoionisation of molecules only first steps have been done sofar and many exciting challenges lie still ahead. One open problem is electron correlation in direct double ionisation of fixed in space $H_{2}$ (Kossmann et al 1989; Reddish et al 1997; Dörner et al 1998a; Reddish and Feagin 1998; Feagin 1998; Walter and Briggs 1999; Walter and Briggs 2000). In this case not only the internuclear axis but also the internuclear distance at the instant of photoabsorption can be measured via the ion fragment momenta. This would, for the first time, allow controlling the initial state in a double ionisation process (Weber 2003). Another very promising route is to take such experiments to the time domain by using pump-probe techniques (Davies et al 2000). Among the open and controversially discussed questions is also the localization or delocalization of vacancies in homonuclear molecules (Pavlychev et al 1998) as well as multiple electron processes manifested in the satellite lines (De Fanis et al 2002).

\subsection{Single and Multiple Ionisation in Intense Laser Fields}

The interaction of intense coherent light with atoms, molecules, clusters or solids has attracted increasing attention in the recent past triggered by the tremendous technical progress in the realization of such radiation. Pulse times below $100 \mathrm{fs}$ and intensities exceeding $10^{16} \mathrm{~W} / \mathrm{cm}^{2}$ are routinely realized nowadays (see e.g. Brabec and Krausz (2000)) using Ti:Sa lasers $(800 \mathrm{~nm}, \hbar \omega=1.5 \mathrm{eV})$. Single electron emission, the intensity dependence of ion rates, multiple ionisation yields, dissociation of molecules or clusters, harmonic generation etc. have been explored in detail over many years essentially using single particle detection techniques (see e.g. DiMauro and Agostini (1995)). A profound theoretical understanding of non-linear multi-photon processes has emerged for reactions that either involve only one electron or for processes where electrons can be considered to act independently within a so-called "single active electron" (SAE) description (see e.g. Protopapas et al (1997)). 
Many-electron dynamics, i.e. the femtosecond time-dependent correlated motion of several electrons and ions in the field, however, which determine for instance pathways to multiple ionisation or the dissociation of molecules and clusters, cannot be treated by ab initio theories up to now. Even helium double ionisation, the most simple two-electron reaction, has remained subject of numerous controversial debates over the last decade (see e.g. Lambropoulos et al (1998)). Experimentally, a break-through has been achieved about three years ago with the first successful implementation of Reaction Microccopes (see Chapter 3) producing data with unprecedented resolution and completeness for many-electron processes.

The present Section tries to summarize new results on the fragmentation of atoms obtained using these next-generation methods (for a recent review on the field see also Dörner et al (2002)), giving only few references on molecular dissociation work. After the introduction, recent data on single ionisation in the tunnelling regime will be described in Subsection 4.2.2 followed by a selection of illustrative results on double ionisation in Subsection 4.2.3 giving reference to available theoretical predictions. Subsections 4.2.4 and 4.2.5 deal with the details of the correlated electronic motion.

\subsubsection{Single Ionisation: Recollision in the Tunnelling Regime}

Single electron emission with eV up to $\mathrm{MeV}$ electron energies as a result of the interaction of intense laser pulses with atoms has been explored in great detail in the past covering a large range of laser intensities. At low intensity, for typical Keldysh (Keldysh 1964) parameters $\gamma=\left(I_{P} / 2 U_{P}\right)^{1 / 2} \geq 1\left(I_{P}\right.$ : ionisation potential; $U_{P}=I /(2 \omega)^{2}$ : ponderomotive potential; $I, \omega$ : laser intensity and frequency), the "quantum-nature" of the field causes a rich structure in the photoelectron spectra due to a variety of processes that might occur, like "above threshold ionisation" (ATI), "channel opening" or "closing", "resonant enhanced ionisation", coherent scattering etc.. At high intensities instead, for $\gamma<1$ where the field might be treated classically, the spectra become increasingly smooth and unstructured. This has been explained early by quasi-static tunnelling (Keldysh 1964; Faisal 1973; Corkum et al 1989; Delone and Krainov 1998) or by treating above threshold ionisation quantum mechanically (the so-called Keldysh-Faisal-Reiss, KFR-model Keldysh (1964); Faisal (1973); Reiss (1980, 1987)). In tunnelling theory, the active electron tunnels through the barrier in a first step and is then accelerated, gaining drift energy in the oscillating field $\vec{E}(t)=\vec{E}_{0} \sin (\omega t)$ in a second step. The drift momentum $P_{d r i f t}\left(t_{0}\right)=(q / \omega) E_{0} \cos \left(\omega t_{0}\right)$ is a smooth function of the tunnelling phase $\varphi=\omega t_{0}$ and is zero for tunnelling at the maximum of the field. The maximum drift energy of 
$2 U_{P}$ is reached for tunnelling at a phase where the field is zero. Immediately, interest concentrated nearly exclusively on large electron energies, beyond $2 U_{P}$, where significant deviations from simple tunnelling as well as KFR-models were recognized and explained by "rescattering" of the oscillating electron at its parent ion (see e.g. Delone and Krainov (1998); Walker et al (1996); Sheehy et al (1998)) causing acceleration up to a maximum energy of $10 U_{P}$.

Recently, the low-energy part of the spectrum has been explored for the first time for single ionisation of $\mathrm{Ne}$ in the tunnelling regime $\left(25 \mathrm{fs}, 1 \cdot 10^{15} \mathrm{~W} / \mathrm{cm}^{2}, 800 \mathrm{~nm}\right.$, linear polarization) at $\gamma=0.39$ by mapping the complete final state momentum space with high resolution in coincidence with $\mathrm{Ne}^{1+}$ ions (Moshammer et al 2003a). As shown in Figure 15, smooth distribution without any indication of an ATI peak structure is observed along the longitudinal $\left(P_{\|}\right)$as well as transverse $\left(P_{\perp}\right)$ momentum directions for the electron (Figure 15a) as well as for the ion (Figure 15b), as expected. Surprisingly however, a distinct minimum at zero electron momentum was found in striking contradiction to the well accepted tunnelling theory. Since tunnelling is most likely at the maximum of the field $\varphi=\pi / 2$ and since those electrons receive zero drift momentum, a Gaussian distribution function is predicted with a maximum of the electron momentum distribution at $P_{e \|}=0$, independent of $P_{e \perp}$.

Quantitatively this is illustrated in Figure 16, where the tunnelling theory result (Delone and Krainov 1991) for $0.7 \cdot 10^{15} \mathrm{~W} / \mathrm{cm}^{2}$ is shown (thin line) along with the experimental data from Figure 15 projected onto the longitudinal axis for all transverse momenta. Also indicated (thick line) is the result of recent semi-classical calculations (Chen and Nam 2002) for an intensity of $0.7 \cdot 10^{15} \mathrm{~W} / \mathrm{cm}^{2}$ and a helium target, clearly showing not only a minimum at $P_{e \|}=0$ but also deviations from the Gaussian shape in qualitative agreement with the experimental data. Here, in order to model single ionisation, electrons are set into the continuum with a velocity distribution obtained from the wave function of the tunnelled electron. Then, the set of trajectories is propagated according to Newton's classical equations in the combined field of the laser and of the Coulomb potential of the residual singly charged helium ion. The latter is also modelled classically assuming the electron to be in the $\mathrm{He}^{+}$ ground state represented by a micro-canonical distribution. Thus, all mutual interactions between all particles are accounted for during the whole laser pulse in all three dimensions.

Within this model both, the minimum at zero momentum as well as the deviations from the Gaussian-shaped tunnelling-theory prediction result from the interaction of the tunnelled elec- 
tron with its parent ion, i.e. are a consequence of rescattering. Electrons tunnelling in the maximum of the field experience multiple recurrences to the parent $\mathrm{He}^{+}$ion with low velocities and, accordingly, large elastic scattering cross sections. Thus, they are effectively redistributed to larger transverse momenta depleting at the same time the intensity at $P_{e \perp}$ close to $P_{e \|}=0$. Switching off the interaction with the $\mathrm{He}^{+}$ion, which can either be represented classically as described above or simply by a screened potential, causes both characteristic features to disappear and the tunnelling-theory result is recovered. Whereas the width of the momentum distribution significantly depends on the intensity, the minimum however is observed for all power densities investigated, ranging from $0.3-1 \cdot 10^{15} \mathrm{~W} / \mathrm{cm}^{2}$.

Thus, high-resolution momentum imaging surprisingly revealed new insight into single ionisation in the tunnelling regime at low electron energies, a process that has been considered to be well understood before. Whereas effects from re-collision have been clearly observed and are commonly accepted to be of decisive importance at high electron energies beyond $2 U_{P}$, recollision has never been discussed in the context of extremely small electron momenta at essentially zero electron energy. Recent purely classical, and, thus, "over-thebarrier" calculations on strong field single ionisation (Feeler and Olson 2000) showed a maximum at zero momentum leading to the conclusion that the dip in the semi-classical theory seems to be closely connected with the quantum nature of the first "tunnelling" step.

\subsubsection{Double Ionisation: Non-Sequential and Sequential}

In Figure 17 momentum distributions parallel $\left(P_{\|}\right)$and perpendicular $\left(P_{\perp}\right)$ to the laser polarization axis are shown for $\mathrm{Ne}^{(1-2)+}$ ions created in intense, linear polarized fields in the sequential and non-sequential regime, respectively at $25 f_{s}$ pulse length (Moshammer et al 2000). Similar data have been published for He by Weber et al (2000) and Ar by Weber et al (2000a) and Feuerstein et al (2001). Note, in disagreement with Figures 15,16 (Moshammer et al 2003a), the momentum distribution for single ionisation displays a maximum at zero momentum. This is due to the limited recoil-ion momentum resolution achieved in these early measurements being not sufficient to observe the dip.

As for single ionisation in the more recent experiments, a pronounced double peak structure along the polarization direction was found for double and triple ionisation in an intensity regime (see (2) in Figure 17) where double ionisation is dominated by non-sequential (NS) mechanisms (Larochelle et al 1998). This structure automatically ruled out "shake-off" (Fittinghoff et al 1992) or "collective tunnelling" (Eichmann et al 2000) as dominant NS double 
ionisation mechanisms terminating a ten years discussion. At the same time, the peaks were found to be compatible with the "antenna" (Kuchiev 1987) or the "recollision" mechanism (Corkum 1993) as was first shown within classical considerations (Feuerstein et al 2000; Moshammer et al 2000; Weber et al 2000). Subsequently, a variety of theoretical predictions (see the detailed discussion and references in Dörner et al (2002)) based on S-matrix theory, on the numerical solution of the time dependent Schrödinger equation (TDSE) as well as on the classical or semi-classical approximations essentially established the double peakstructure and recollision as the dominant NS double ionisation mechanism (see also earlier measurements with circularly polarized light by Fittinghoff et al (1994) and Dietrich et al (1994)). In the sequential regime a broader distribution is observed with a single peak at zero momentum along the field axis (see (3) in Figure 17).

Interestingly, the ability of the experiments to distinguish between different ionisation mechanisms relies on the fact that the ion momenta can, under certain conditions, provide information on the phase $\varphi=\omega t_{0}$ of the time-dependent laser field $\vec{E}(t)=\vec{E}_{0} \sin (\omega t+\varphi)$ where (multiple) ionisation took place, i.e. at what phase the ion was born. This becomes obvious within the classical treatment (Feuerstein et al 2000; Moshammer et al 2000; Weber et al 2000; Chen et al 2000) but is also inherent to all quantum calculations listed above. As mentioned before, a charged particle created with charge $q$ and zero initial momentum at a time $t_{0}$ in a pulse that is long compared to the oscillation period ( $\tau \omega>>1 ; \tau$ : pulse duration), gains a final drift momentum parallel to the laser field that only depends on the phase. At a laser frequency of $\omega=0.05 \mathrm{a} . u$. and a field amplitude $E(t)=0.18 \mathrm{a} \cdot u \cdot\left(1 \cdot 10^{15} \mathrm{~W} / \mathrm{cm}^{2}\right) \mathrm{a}$ typical momentum resolution for singly charged ions of 0.1 a.u. thus, would translate in a phase resolution of about $3 \%$ of a full optical cycle i.e. to a time resolution of 80 as .

The "tracing" of the phase by measuring the ion drift momentum is disturbed by any momentum transferred to the ion when it is created by a certain process like "tunnelling", multiphoton absorption, "rescattering" or excitation during rescattering plus subsequent tunnelling ionisation of the excited electron. This leads to a reduced, nevertheless still sufficient phase resolution for the neon target, as is impressively demonstrated for NS double ionisation: "Rescattering", where double ionisation is due to an ionising collision between the first electron being accelerated in the laser field and thrown back on its parent ion, mainly occurs at a phase where the electric field is close to zero i.e. at $\omega t_{0}=n \cdot \pi(\mathrm{n}=1,2, \ldots)$ resulting in a large drift momentum of the doubly charged ion. In contrast, zero average drift momentum is expected to dominate for the other two NS double ionisation mechanisms that have been pro- 
posed, "shake-off" and "collective tunnelling", since both most likely occur at maximum field strength, i.e. at phases $\omega t_{0}=(2 n+1) \cdot \pi / 2(\mathrm{n}=0,1,2, \ldots)$. The various mechanisms can only be distinguished in an unambiguous way by an ion momentum measurement alone, if the momentum transfer during creation of the ion by the one or other mechanism is less than the differences in ion drift momenta resulting from the average phase differences where the processes occur.

\subsubsection{Correlated Motion of Electrons in the Sequential and Non-Sequential Regimes}

Subsequent experimental and theoretical work started to explore the correlated motion of the emitted electrons. In Figure 18, for example, the correlated momenta of two emitted electrons are shown along the field direction $\left(k_{e z i}=P_{e i \|}\right)$ for double ionisation of Ar in the nonsequential $\left(3.8 \cdot 10^{14} \mathrm{~W} / \mathrm{cm}^{2}\right.$, upper pannel) as well as in the sequential regime $\left(1.5 \cdot 10^{15} \mathrm{~W} / \mathrm{cm}^{2}\right.$, lower pannel), respectively, first published by Weber et al (2000b). Whereas the electrons were found to behave independently for sequential ionisation, peaking at zero drift momentum each (Figure 18b), a strongly correlated behavior is visible in the NS regime with a pronounced maximum for both electrons being emitted into the same hemisphere with very similar momenta (Figure 18a). The authors explained the location of this maximum in a simple rescattering scenario where the returning electron excites the remaining ion which then is immediately field ionised.

Pure collisional ionisation during rescattering, essentially without the assistance by the laser field, seems to be responsible for Ne double ionisation as illustrated in Figure 19a. As discussed by Moshammer et al (2003), for example, recollision happens at a well defined energy $E^{\text {recoll }}$ and phase $\omega t_{1}$ for a certain tunnelling phase of the first electron $\omega t_{0}$. After

recollision, each electron obtains a well defined and equal drift momentum of $P_{e i \|}^{d r i f t}\left(\omega t_{0}\right)$. In addition, they can share the excess energy $E^{e x}=E^{\text {recoll }}-I_{P}\left(I_{P}\right.$ : ionisation potential). Neglecting the transverse energies of the electrons, energy conservation $P_{e\|\|}^{2}+P_{e 2 \|}^{2}=2 E^{e x}$ yields a circle with radius $\left(2 E^{e x}\right)^{1 / 2}$ around the points $\left( \pm P_{e 1 \|}^{d r i f t}\left(\omega t_{1}\right), \pm P_{e 2 \|}^{d r i f t}\left(\omega t_{1}\right)\right)$ as maximum possible drift momentum combination. Considering all phases, the full curved lines in Figure 19a for $\mathrm{Ne}$ were calculated to represent the classical boundaries, defined by momentum and energy conservation during rescattering, inside which all correlated events must occur. 
While the comparison with the experimental data impressively shows that nearly all events are within these boundaries for the Ne target, the correlation pattern itself, however, with many electrons of quite similar longitudinal momenta, is not reproduced by calculations based on field-free electron-impact (e,2e) ionisation dynamics. Similar as in field-free collisions, unequal energy sharing between both emitted electrons has been calculated to be the most likely situation at not too low intensities, resulting in unequal final momenta for NS double ionisation of $\mathrm{Ne}$ at $1 \cdot 10^{15} \mathrm{~W} / \mathrm{cm}^{2}$ (Goreslavskii and Popruzhenko 2001). Several other calculations, using a hard core form factor for the electron collision, solving the onedimensional TDSE with correlated electrons, using an S-Matrix approach (Becker and Faisal 2000, 2002) or semi-classical methods (Chen et al 2000) seem to be not in accordance with the present experimental results. However, most theoretical work was devoted to helium whereas, until now, no such experimental data have been reported. Hence, final conclusions have to await further clarification. Nevertheless, recent calculations within the S-Matrix approach by Becker and Faisal (2002) considering the shake-off amplidtude separately resulted in two electrons emerging with very small momenta, i.e. strongly peaked at the origin in Figures 18,19 and, therefore, support the above argument.

For both argon measurements shown in Figure 18a (Weber et al 2000b) and Figure 19b (Feuerstein et al 2001), the classically allowed direct ionisation regime without any assistance of the field shrinks significantly due to the lower intensity. At $2.5 \cdot 10^{14} \mathrm{~W} / \mathrm{cm}^{2}$ (Figure 19b) it is completely restricted inside the full lines i.e. to the quadrants with equal emission direction of both electrons with equal signs of both momenta. A large yield of electrons being emitted into opposite hemispheres has been interpreted as excitation during recollision of the still bound electron which then might be field-ionised in one of the subsequent maxima of the oscillating field (Feuerstein et al 2001). Note that direct field ionisation "at the instant" of recollision leads to emission into the same hemisphere (Weber et al 2000b). In contrast to $\mathrm{Ne}$, the argon $3 p$ to $3 \mathrm{~d}$ excitation cross sections are large making such a scenario quite likely as will be discussed in more detail in the next Subsection. Observed momenta were within the classical kinematical limits (broken lines) for this process which tends to "fill the valley" inbetween the two "recollision maxima" in the ion-momentum distribution (projection onto the diagonal from the lower left to the upper right in Figure 19, see Feuerstein et al (2001)).

In summary, even if the overall structure of novel experimental data obtained using Reaction Microccopes strongly support that rescattering is the dominant NS double ionisation mechanism at least for the neon target, the correlated motion of both electrons in the field is far from being consistently understood theoretically. 


\subsubsection{Final State Coulomb Repulsion and Sub-Threshold Recollision at Low Intensity}

Preferred emission of two electrons with nearly identical momenta along the field direction as observed in the experiment for neon targets seems to contradict electron-repulsion in the final state. Quantum mechanical calculations solving the one-dimensional TDSE on a grid with fully correlated electrons in the helium atom (Figure 20 left) yielded a minimum for equal $P_{e i \|}$ (Lein et al 2000). Here, however, the Coulomb repulsion is certainly overestimated due to the one-dimensionality of the model. Experimentally, one can artificially reduce the dimensions in the final state by the requirement, that the final transverse momentum of one of the electrons - and thus, in an (e,2e) scenario also of the second one - is small. Then, as illustrated in Figure 20 (right), the maximum along the diagonal with equal $P_{e i \|}$ was found to be significantly depleted (Weckenbrock et al 2001; Moshammer et al 2002). The electrons are restricted in their transverse motion and, consequently, repel each other more strongly longitudinally.

Very recently, recoil-ion momentum distributions have been measured for $\mathrm{Ar}^{2+}$ and $\mathrm{Ne}^{2+}$ ions at very low intensities extending down to maximum recollision energies $E^{\text {recoll }}=3.17 \cdot U_{P}$ well below the ionisation potential $I_{P}$ of ground-state $\mathrm{Ar}^{+}$or $\mathrm{Ne}^{+}$ions in a field-free environment (Eremina et al 2003). In Figure 21, they are shown for various ratios of recollision energies to ionisation potentials $E^{\text {recoll }} / I_{P}$ ranging from values larger than one, where classical field-free recollision impact ionisation is possible, to $E^{\text {recoll }} / I_{P}$ as low as 0.5 , where the energy of the re-colliding electron is well below threshold. The smooth transition between both regimes observed in the ion rates has always been an argument against a simple recollision scenario to explain NS double ionisation. The observed momentum distributions for $\mathrm{Ar}, \mathrm{Ne}$ and $\mathrm{He}$ at equal $E^{\text {recoll }} / I_{P}$ strongly differ from each other indicating first, that different mechanisms are active below and above threshold for the various species. Whereas the valley between the maxima is more and more filled for Ar finally displaying one single maximum at zero drift momentum for $E^{\text {recoll }} / I_{P}=0.5$, a clear double peak structure remains for Ne even at $E^{\text {recoll }} / I_{P}=0.7$. At the same time there is only one single peak for the helium target directly at threshold evolving only slowly into a quite weak double hump structure at relatively high intensities of $E^{\text {recoll }} / I_{P}=2.3$.

While for argon the transition can be understood in terms of the increased importance of excitation during recollision followed by field ionisation at lower intensities along the lines dis- 
cussed above, smoothly "filling the valley", the pronounced sub-threshold double-hump structure for neon double ionisation was explained as increased importance of recollision at phases of the field, where it is not zero (Eremina et al 2003). Here, the effective ionisation potential of $\mathrm{Ne}^{1+}$ is lowered to such an extent that the re-colliding electron can still ionise the $\mathrm{Ne}^{+}$ion assisted by the field. In such a scenario, more and more but smoothly, different but welldefined and narrow recollision phase intervals become important leading to well defined but lower recoil-ion momenta and, thus, to a pronounced double-peak structure.

Helium (left row in Figure 21) finally, behaves very much like argon with a single peak at zero longitudinal momentum even above the recollision threshold making the whole scenario quite confusing on first glance. It has been shown recently (Bastos de Jesus et al 2003), however, that the different behavior of the various rare gas targets can be consistently explained in a quantitative way by taking into account the ratio of electron impact excitation to ionisation cross sections for the singly charged ions. It turns out, that neon plays a special role in that this ratio is exceptionally small compared to the other elements. Accordingly, excitation during recollision followed by field ionisation of the excited electron is a small channel compared to direct electron impact ionisation during rescattering and, thus, the double hump structure prevails to intensities even below the field-free recollision threshold. The quantitative interpretation given by Bastos de Jesus et al (2003) was based on estimates of the above ratio on the basis of empirical models used in plasma physics taking into account all recollision energies and relative intensities, calculated from the tunnelling phases for the electron weighted with the tunnelling probability, and assuming that the excitation/fieldionisation channel leads to a maximum of the recoil-ion momentum distributions at zero.

Nevertheless, more experimental results for helium, which is the only system where fully quantum-mechanical calculations might be expected within the near future (Parker et al 2001) are urgently needed.

\subsubsection{Transverse Momentum Exchange}

The electron and ion momenta in the direction of the laser polarization which have been discussed so far are mainly a result of the accleration in the field. This is what allows one to use them to gain inside in the time evolution of the ionisation process. The momentum components of all particles perpendicular to the field in contrast are solely a result of the few body momentum exchange. Therefore, the subtleties of the momentum exchange in the recollision event are in the perpendicular plane, not masked by the large momentum transfer from the field. Figure 22 shows the momentum correlation between the two electrons in the plane per- 
pendicular to the polarization (Weckenbrock et al 2003). The first electron is emitted upwards along the arrow; the momentum of the second electron is shown. Both electrons are found to be emitted slightly back to back. The momentum distribution of the doubly charged ion is very similar and also slightly opposite to one electron (not shown). At first glance one might argue that such behavior is simply a result of a binary momentum exchange between the resscattered and the second electron to be kicked out. Astonishingly, S-Martix calculations proof this simple mechanism wrong and suggest that the back-to-back emission in the perpendicular plane is not a result of the binary momentum exchange in the recollision event but is created after the second electron is set free by the long range final state interaction. Figure $22 \mathrm{~b}$ shows the full S-Matrix calculation including binary momentum exchange and final state interaction while in Figure 22c the final state interaction is neglected but the recollision momentum exchange is still active. The same calculation however does not reproduce the observed ion momenta (not shown, see Weckenbrock et al 2003). At present, the study of the transverse momentum exchange highlights once more how far from final conclusions the dynamics of nonsequential double ionisation really is.

\subsection{Electron Impact Ionisation}

While for ion impact kinematically complete experiments for single ionisation could be realized only with the advent of recoil ion momentum spectroscopy, for electron impact first so-called (e,2e) experiments were performed already in the late sixties by Ehrhardt et al (1969) and others. These and the then following numerous (e,2e) experiments contributed substantially to our understanding of the dynamics of the three-body Coulomb problem. On the other side due to the small acceptance angle this technique reaches its limits for multiple coincidence experiments as they are required for the study of double ionisation, excitationionisation and molecular fragmentation. This is also the case for experiments requiring short pulsed projectile beams as e.g. the study of electron collisions which take place in strong light fields. The light intensities required for such laser-assisted collisions $\left(I>10^{12} \mathrm{~W} / \mathrm{cm}^{2}\right)$ can be realized only by pulsed laser beams.

Therefore, despite the huge amount of data which has been collected using conventional electron spectroscopy the application of the Reaction Microscope enables a variety of new and unprecedented experiments for electron collision processes. This is due to the large phase space acceptance of the spectrometer, the strongly relaxed beam time restrictions for simple electron sources compared to synchrotron or ion beam facilities and the pulsed-mode opera- 
tion of the projectile beam which allows synchronization with other beams as e.g. short laser pulses. In the following a few examples which have been realized recently or are under way are presented.

\subsubsection{Single and Double Ionisation}

The first measurements using recoil ion momentum spectroscopy for electron impact single and double ionisation were performed by Jagutzki et al (1996). The electrons in the final state were not detected. From the good agreement of the recoil-ion momentum distribution with experimental and theoretical electron momentum distributions for single ionisation the authors concluded, that collisions with small momentum transfer dominate. For double ionisation the recoil ion momentum distribution was observed to be much broader. This was attributed in first instance to the broader two-electron Compton profile. The authors pointed out that kinematically complete measurements for double ionisation (so-called (e,3e) experiments) would become feasible if two final state electrons would be observed in coincidence with the recoiling ion. Such experiments were realized in the following using a Reaction Microscope for the additional detection of one or two slowly ejected electrons for single or double ionisation, respectively (Dorn et al 1999).

Using the combined recoil-ion and electron momentum spectrometer which is briefly described in Section 3.1 for electron projectile beams is not straight forward. In contrast to essentially all other projectiles used in connection with Reaction Microccopes (ions, synchrotron and laser photons) electrons are strongly deflected by the magnetic field which serves to guide the secondary electrons to the detector. The configuration with the projectile beam collinear to the magnetic field lines which would be optimal for guiding the projectile electron beam into the target is not practicable since in this case the primary electrons would impinge on the electron detector after passing the target. Despite the small beam current which is in the order of $60 \mathrm{pA}$, this would correspond to a few hundred $\mathrm{MHz}$ count rate, a value far beyond the dynamical range of the detector. Therefore, a different operation mode was used where the primary beam emitted on the apparatus axis is deflected off the axis and going trough a full cyclotron revolution before returning to the axis in the target volume (Dorn et al 2002). After another half of a complete revolution it has the maximum deviation from the spectrometer axis and therefore it passes by the electron detector and is dumped in a Faraday cup. While this arrangement enables experiments for electron impact using the Reaction Microscope, the projectile velocity determines the required magnetic field strength. Thus, the highest energy of the ejected electrons which is accepted over the full solid angle is restricted. For 
$E_{0}=2000 \mathrm{eV}$, the required field of $B=12$ Gauss results in full efficiency for electrons up to $25 \mathrm{eV}$ only. Nevertheless, with the restriction that free zooming of the fragment particle imaging properties is not possible, kinematically complete experiments for electron impact can be performed. In future this limitation will be removed by using an electron detector with a central bore which allows one to reach the ideal geometry for the projectile beam trajectory which will be on axis of the magnetic field $\vec{B}$ and therefore $\vec{B}$ and the projectile energy $E_{0}$ will be decoupled.

The large phase space acceptance of the apparatus is demonstrated in Figure 23 for single ionisation. In accordance with most $(\mathrm{e}, 2 \mathrm{e})$ experiments performed with conventional electron spectrometry only events with coplanar geometry are displayed for which the ejected electron moves in the scattering plane defined by the incoming and scattered projectile. The cross section is plotted as function of the electron emission angle $\Theta_{b}$ (x-axis) and, simultaneously, as function of the momentum transfer $|\vec{q}|$ (y-axis). This representation nicely reveals the behavior of the so-called binary and the recoil peak, namely their magnitude and angular position in dependence on the momentum transfer. The binary peak around $\Theta_{b} \approx 60^{\circ}$ is generally attributed to a binary knock-out collision where the target electron is emitted along $\vec{q}$, while for the recoil peak the ejected electron undergoes an additional scattering process in the ionic potential and is emitted into the backwards direction $-\vec{q}$ around $240^{\circ}$. The kinematical shift of the cross section maxima to smaller $\Theta_{b}$ for decreasing $|\vec{q}|$ is visible as well as the known behavior, namely that the recoil peak decreases much faster in intensity compared to the binary peak for increasing momentum transfer. Although the data shown represent only a small fraction of the full final state phase space covered experimentally it would constitute a large number of experimental runs for a conventional set-up. Furthermore these do not allow covering the full angular range of $\Theta_{b}$ from $0^{\circ}$ to $360^{\circ}$. In Figure 24 the momentum distribution of the ejected electron is presented for fixed momentum transfer of $|\vec{q}|=0.6$ a.u. revealing a strong binary emission peak along $\vec{q}$ and a weaker recoil peak in the opposite direction. The momentum distribution around the tip of the arrow indicating the size and direction of the momentum transfer represents essentially the bound electrons Compton profile.

The logical next step which is of prime importance for the understanding of the correlated electron dynamics is the kinematically complete investigation of electron impact double ionisation. The first (e,3e) experiment was performed by Lahmam Bennani et al (1989) for the 
argon target. First experiments for the fundamental helium target were performed essentially simultaneously with conventional electron spectrometry for $E_{0}=5500 \mathrm{eV}$ by Taouil et al (1998) and with the Reaction Microscope by Dorn et al (1999) for $E_{0}=3000 \mathrm{eV}$. While the former, conventional experiment was restricted to a single projectile scattering angle close to the optical limit $(|\vec{q}|=0.24$ a.u. $)$, to a particular energy of the ejected electrons $\mathrm{e}_{\mathrm{b}}$ and $\mathrm{e}_{\mathrm{c}}$ ( $E_{b}=E_{c}=10 \mathrm{eV}$ ) and to coplanar scattering geometry, the latter experiment allowed a global view of the final state momentum space. While the statistics of these first measurements did not allow definite statements for the fully, fivefold differential cross section (FDCS) new insight into the DI collision could be obtained for partially integrated cross sections. Examining the angular correlation of the ejected electron pair, both experiments could confirm close similarities to photo double ionisation for small momentum transfer with the most probable relative emission angle of $135^{\circ}$. For larger $|\vec{q}|$, smaller relative angles of the ejected electron pair were observed by Dorn et al (1999) which was interpreted as a signature of a first order binary collision process. In subsequent experiments for $E_{0}=2000 \mathrm{eV}(v=12 a . u$. $)$ FDCS could be obtained which allowed a detailed examination of the cross section and quantitative tests of theoretical calculations. (Dorn et al 2001, 2002). For $v=12$ a.u. the projectile generally can be treated as a small perturbation. On the other hand the ionised electrons most probably are slow and, therefore, they strongly interact with each other and the residual ion. The examination of the FDCS pattern reveals that the subtle details of this correlated emission strongly depend on the amount of momentum $|\vec{q}|$ transferred by the projectile to the target. In the optical limit, namely for small projectile scattering angle and therefore small $|\vec{q}|$, the cross section pattern is dominated by dipole selection rules and the strong Coulomb repulsion of the electrons. This is illustrated in Figure 25a where the FDCS is plotted for $|\vec{q}|=0.5$ a.u. . Events are chosen where the ionised electrons have energies $E_{b}$ and $E_{c}$ of $5 \mathrm{eV}$ each and move in the projectile scattering plane. The FDCS is plotted versus the emission angles $\Theta_{b}$ and $\Theta_{c}$ of the ejected electrons with respect to the incoming projectile beam forward direction. The solid circular lines in the diagrams indicate the angular range accessible by the apparatus. For angular combinations outside the circles the arrival time difference of the electrons on the detector is less than the detector dead time of $15 \mathrm{~ns}$ and, therefore, the second electron is not registered. The cross section shows a pronounced structure with four strong peaks indicating that particular angular combinations $\left(\Theta_{b}, \Theta_{c}\right)$ are strongly favoured. The agreement with photo- 
double ionisation data shown in Figure $25 \mathrm{~b}$ is very good if the light polarization axis $\vec{E}$ is aligned along the corresponding momentum transfer direction $\vec{q}$. Peak (A) in Figure 25a corresponds to emission as sketched in Figure 25b with the sum momentum of the ionised electrons going along $\vec{q}$. Respectively, peak (B) corresponds to emission with the sum momentum of the electrons being reversed. For equal energy sharing the cross section is symmetric with respect to the main diagonal of the diagram since both electrons are indistinguishable. Dipole selection rules suppress electron emission along the dashed and dotted lines. In addition, electron-electron repulsion gives rise to the broad cross section minimum around $\Theta_{b}=\Theta_{c}$. Deviations of the electron impact data from the strict symmetries visible in Figure $25 \mathrm{~b}$ for photo double ionisation are due to the finite momentum transfer $|\vec{q}|$ which can lead to differences in shape and intensity of peak (A) and (B). The shift of peak (B) to the top right part of the diagram is due to contributions of higher order collisions.

While various successful theoretical models exist to describe photo double ionisation, which has been attributed partly to the strong restrictions imposed by selection rules (Briggs and Schmidt 2000), theory is in a far less developed shape for electron impact double ionisation. In Figure 25e theoretical results obtained with the convergent close coupling (CCC) approach are shown (Dorn et al 2001). While this method treats the interaction of the two slowly ejected electrons non-perturbatively, the projectile-target interaction is described in first order by applying the first Born approximation. Comparing with the experimental cross section the agreement is reasonably good in shape and in the relative peak heights. Clearly, the finite momentum transfer gives rise to higher multipole contributions to the cross section which can be identified as finite cross section for angular combinations where the dipole selection rules would strictly enforce a zero value, namely at the intersection points of the dashed and dotted lines. Disagreement between experiment and theory is present for the node for back-to-back emission (dashed lines) which differently from theory is partially filled in the experimental data. A further disagreement is the shift of the peaks marked (B) to larger angles for both emitted electrons. As mentioned above this can be explained by higher order projectile-target interactions which are not within the scope of a first Born treatment.

The fragmentation pattern is strongly modified if collisions with larger momentum transfers are investigated where dipole selection rules do not act (Dorn et al 2002). For $|\vec{q}|=2 a . u$. and low energies of $E_{b}=E_{c}=5 \mathrm{eV}$ the repulsion of the ejected electrons is dominant and gives rise to a Wannier-like back-to-back emission. For larger energies of $E_{b}=E_{c}=20 \mathrm{eV}$ a cross 
section peak is observed for conditions where the Bethe ridge condition is fulfilled: the full momentum transfer $\vec{q}$ is carried by the ejected electron pair while the residual ion has only little momentum. This peak was attributed to be mainly due to the two-step 1 (TS1) ionisation mechanism where the electron ionised in a first binary collision with the projectile collides on the way out of the atom with the second electron. This picture is also consistent with the observed most probable relative ejection angle close to $90^{\circ}$.

For a quantitative comparision with theory a particular cut of the experimental data is shown in Figure 26 where the emission angle of one ejected electron $\left(E_{b}=25 \mathrm{eV}\right)$ is fixed to $\Theta_{c}=0^{\circ}$ and the emission angle of the second electron $\left(E_{c}=5 \mathrm{eV}\right)$ is scanned (Dorn et al 2002a). The calculations shown, the Convergent Close Coupling theory (CCC, solid line) and the calculation using the analytical $3 \mathrm{C}$ or BBK wave function (dashed line) differ strongly from each other and only the CCC calculation is in fairly well agreement with the experiment. This demonstrates that the $(\mathrm{e}, 3 \mathrm{e})$ reaction in particular in the large momentum transfer regime where no dipole selection rules act is a critical test for theory.

From the above discussion the expectation is confirmed that fast collisions can be understood essentially in terms of a first order projectile-target interaction with only minor contributions from higher-order processes. This is expected to change for reduced projectile velocity and therefore increased perturbation. Indeed, results for $500 \mathrm{eV}$ electron impact ( $v=6$ a.u.) show strongly modified cross sections in particular for low momentum transfer (Dorn et al 2003). In Figure 27 an example is shown for $|\vec{q}|=0.6$ a.u. and the same energies of the ejected electrons as for Figure $23\left(E_{b, c}=5 \mathrm{eV}\right)$. There is no resemblance with the photo ionisation pattern of Figure $25 \mathrm{~b}$ any more and the symmetry with respect to the momentum transfer direction present for fast collisions is completely lost. This indicates that second order collisions or the so-called two-step 2 (TS2) mechanism is important. Therefore, any calculation describing the collision in first order must fail. First attempts to reproduce these data with a CCC calculation including the second Born amplitude are encouraging (Dorn et al 2003). On the other hand it is to be expected that in general a perturbation expansion of the exact transition amplitude fails if the higher order contributions become of comparable magnitude as the first order contribution. In future investigations of $(e, 3 e)$ reactions in the non-perturbative regime are envisaged where all three final state electrons have comparable velocity. Then, the full complexity of the four body dynamics is expected to show up.

\subsubsection{Ionisation-Excitation}


Simultaneous excitation of one target electron and ionisation of a second electron in many aspects resembles the double ionisation process. In both reactions electron correlation and higher order projectile-target interaction play a dominant role. On the other hand, for excitation-ionisation the final state contains only two continuum electrons, compared to three for double ionisation. Therefore, the theoretical treatment is much simpler since in particular for very asymmetric energy sharing reactions, calculations can neglect the long range final state interaction of the continuum electrons and restrict to modelling the short range collision dynamics in a more accurate way. On the experimental side, up to now there is no "perfect" $(e, \gamma 2 e)$ experiment (Balashov and Bodrenko 1999) which would imply to determine the ionisation process kinematically complete and to measure simultaneously the excitation amplitudes and their relative phases for the excited ionic state. For the helium target such an experiment could be performed for the $\mathrm{He}^{*+}(\mathrm{np})$ final state if both continuum electrons and the photon emitted in the decay are detected. In addition, the measurement of the polarization parameters or of the angular distribution of the photons is required. So far such a triple coincidence detection was not feasible and experiments were restricted to record only two final state particles, either two electrons (Avaldi et al 1998; Dupré et al 1992) or one electron and the photon (Hayes and Williams 1996; Dogan and Crowe 2002), integrating over the observables of the particle which had not been observed.

Recently a triple coincidence $(e, \gamma 2 e)$ experiment has been performed, using a Reaction Microscope that was additionally equipped with two large area MCP detectors for the detection of VUV photons (Dorn et al 2002a). The following reaction was investigated:

$$
e^{-}\left(E_{0}=500 e V\right)+H e\left(1 s^{2}{ }^{1} S\right) \rightarrow 2 e^{-}+H e^{+^{*}}\left(n p{ }^{2} P\right) \rightarrow 2 e^{-}+H e^{+}\left(1 s^{2} S\right)+\gamma(\lambda \leq 30.4 n m)
$$

Experimentally, a slowly ejected electron, the recoiling singly charged ion and the fluorescence photon emitted by the residual ion were detected in a triple coincidence. The experimental resolution for the Q-value of the reaction did not allow resolving the different excited np-states. Thus, despite the fact that the $\mathrm{He}^{+}(2 p)$ state gives the main contribution with more than $80 \%$ relative probability, the experiment so far is not fully resolved what the final state is concerned. For reduced projectile energies around $100 \mathrm{eV}$ which are envisaged for the future, the Q-value resolution will allow to separate the $2 \mathrm{p}$ state from higher states.

In Figure 28 transversal momentum distributions for electrons and ions are shown for the $(e, \gamma 2 e)$ reaction and compared with corresponding distributions for $(\mathrm{e}, 2 \mathrm{e})$ and $(\mathrm{e}, 3 \mathrm{e})$ processes, respectively. All three experiments have been performed for $E_{0}=500 \mathrm{eV}$ and with identical values for the extraction fields at the Reaction Microscope. 
While the observed recoil-ion momentum distribution for $(e, \gamma 2 e)$ is very similar to the one for (e,3e), which one might have expected, significant differences occur in the electron momentum distribution which is closer to the one for $(e, 2 e)$. This clearly indicates, that different dynamic mechanisms dominate for $(\mathrm{e}, 3 \mathrm{e})$ compared to $(e, \gamma 2 e)$ which is quite surprising. Presently the data are further analyzed in order to extract higher differential cross sections which might possibly clarify the reasons for the observed differences.

\subsubsection{Laser Assisted Collisions}

Laser assisted collisions between atoms and charged particles are interesting from different points of view: First, as discussed in Section 4.2, these processes play a central role in the double ionisation reaction induced by ultra-short fs-laser pulses in the non-sequential intensity regime. Here, an electron ionised in the laser pulse is accelerated in the oscillating electric field and re-collides with the parent ion giving rise to the emission of a second target electron. It is expected that laser assisted (e,2e) experiments where the collision parameters are under full control give detailed insight into this process. Second, it might be possible to control the interaction pathways in ion-atom collisions, i.e., to enhance the population of favoured final states and to suppress the production of undesired ones by a suitable choice of the laser parameters (Kirchner 2002). This might be a first step to control molecular reactions. Third, there are a number of theoretical studies aiming at modeling the laser assisted ionisation process in different approximations (see e.g. Khalil et al 1997). Phenomena which have to be considered are the dressing of the initial and final target states as well as the interaction of the charged continuum particles with each other and the laser field. The electromagnetic field can act as a reservoir of energy and its polarization vector introduces a new axis of symmetry. As a result, the $(e, 2 e)$ cross sections for laser assisted collisions differ strongly in shape and magnitude from the field free cross sections as predicted in several theoretical papers on this topic (Khalil et al 1997, Makhoute et al 1999, 2002). On the other hand little is known about the validity and the accuracy of these treatments simply due to the lack of experimental data. Experiments with the aim to realize such investigations are presently under way using a Reaction Microscope. In this set-up a pulsed Nd:Yag laser beam $(\lambda=1064 \mathrm{~nm}$, pulse duration $\tau_{l}=7 \mathrm{~ns}, I \approx 10^{12} \mathrm{~W} / \mathrm{cm}^{2}$ ) has been synchronized in time and collinearly superimposed in space with the pulsed projectile electron beam $\left(E_{0}=1000 \mathrm{eV}, \tau_{e}=1.5 \mathrm{~ns}\right)$ at the position of a target gas jet. By alternating measurements with and without the laser beam kinematically complete data can be obtained for the laser assisted process as well as for the field free case. 
As an example in Figure 29 the longitudinal momentum distribution of the residual ions is shown for electron impact ionisation of helium. Compared to the field-free situation without the laser field the cross section for laser assisted collisions is increased significantly at small

momentum transfers for $\left|P_{\|}\right|<0.4 a . u$. On the other hand for $\left|P_{\|}\right|>0.4 a . u$. the cross section is unchanged. This can be understood in terms of a two-step process where the helium atom is excited in a first step by electron impact to $\mathrm{He}^{*}(1 \mathrm{~s} \mathrm{nl})$ states and the excited state is subsequently ionised by the laser radiation with a photon energy of $1.1 \mathrm{eV}$, a process which has been discussed and seems to strongly contribute in double ionisation of argon and helium in strong laser fields (see Section 4.2). For this photon energy, ionisation by a single photon is possible for $n=4$ and higher principal quantum numbers. Since the longitudinal ion momentum distribution essentially reflects the Compton profile of the bound electron, the narrow momentum distribution is consistent with photo ionisation of excited states. The transversal momentum distribution on the other hand is observed to be broad due to the momentum transfer of the scattered projectile.

This two-step process might open the novel possibility to investigate even excitation processes in a kinematically complete way using the Reaction Microscope by controlled ionisation of the excited state after its creation. For the highest intensities reached so far of $4 \cdot 10^{12} \mathrm{~W} / \mathrm{cm}^{2}$ the relative increase of the ionisation probability with the laser beam on is about a factor of two higher than expected for the discussed two-step model. This can be due to high-intensity phenomena which are the object of interest of this investigation. More detailed results are expected if ejected electron momentum distributions are analyzed in addition to the ion momentum distributions.

\subsection{Ion Collisions}

Originally, recoil-ion momentum spectroscopy and finally Reaction-Microscopes have been developed with the intention to investigate the dynamics of ion-atom collisions. Therefore, an enormous amount of experimental data exists using ions as projectiles. From the kinematical point of view the most simple reactions are bound to bound one-electron transitions from the target to the projectile (single electron capture) because in this case only two particles are in the final continuum sate. The first high resolution capture measurements, where the finally populated electronic states of the projectile could be distinguished by means of a recoil-ion longitudinal momentum measurement, succeeded in the mid nineties (Mergel et al 1995, Wu et al 1995, Cassimi et al 1996) using a cold supersonic jet as a target (COLTRIMS). Since 
then the technique has been improved considerably with respect to both, the quality of the target and the spectrometer performance (see also Dörner et al 2000). As a result, capture measurements with unsurpassed recoil-ion momentum resolutions have been reported using atoms trapped in a MOT as a target (Flechard et al 2001, Turkstra et al 2001, van der Poel et al 2001). Recently, results for electron capture accompanied with excitation of a bound electron of either the target (Weber et al 2001) or the projectile (Kamber et al 2000, Fischer et al 2002) have been published. With highly charged projectiles at low collision velocities (below $10 \mathrm{keV} / \mathrm{u}$ ) the simultaneous capture of several electrons by the projectile contributes considerably to the total cross section. Double and triple electron capture from the target into bound (excited) states of the projectile were studied (for recent results see e.g. Flechard et al 2001a, Zhang et al 2001, Abdallah et al 1998a) or even in coincidence with Auger-electrons, which are emitted from the highly excited projectile after the collision (Hasan et al 1999).

At high velocities (above $100 \mathrm{keV} / \mathrm{u}$ ) electron capture is often associated with the simultaneous ionisation of another target electron (transfer ionisation). Here, the application of COLTRIMS enabled the disentanglement of different reaction pathways (Mergel et al 1997, Schmidt et al 2002). A selection of some of the most recent results and developments concerning electron capture reactions will be presented in the next Subsection 4.4.1. We will then report on the latest data on projectile ionisation in Subsection 4.4.2, another subject where measurements with Reaction-Microscopes made significant contributions to our understanding of the reaction dynamics. Though one-electron transitions, i.e. those where the projectile is ionised or excited only, are not accessible because no target ion is produced. Experiments on the ionisation of both, the projectile and the target, however, allowed to separate different reaction mechanisms which are not to distinguish by means of other methods (Dörner et al 1994, Wu et al 1994, Kollmus et al 2002).

Finally, emphasis is given to studies of single and multiple target ionisation using ReactionMicroscopes. Over the last ten years many data have been published covering a large range of projectile energies and charge states, from $\mathrm{keV} / \mathrm{u}$ protons and low charged ions (Dörner et al 1996a, Abdallah et al 1998, see also Dörner et al 2000) via MeV/u velocities (Jardin et al 1996, Unverzagt et al 1995, Schulz et al 1999, Weber et al 2001) up to GeV/u bare uranium (Moshammer et al 1997). In several experiments the kinematically complete information has been obtained for single (Weber et al 2000c, Moshammer et al 2001), double (Perumal et al 2002, Fischer et al 2003) and even triple ionisation (Schulz et al 2000) of the target atom. Fully differential cross sections for ionisation by ion impact are at hand for the very first time (Schulz et al 2003), which serve as ultimate tests of theories. Some of the most recent results 
in the two regimes, i.e. ionisation at low and at high velocities, respectively, where ionisation proceeds via completely different reaction mechanisms, will be reviewed in the last two Subsections of section 4.4 .

\subsubsection{Electron capture: Dynamics and Spectroscopy}

In capture reactions, i.e. when the electron is bound in the initial target as well as in the final projectile state, the $Q$-value of the reaction is accessible by the measurement of the longitudinal momentum $\mathrm{P}_{\mathrm{R} \|}$ of the recoiling target ion (see Section 2.2). The discrete values of electronic binding energies in both projectile and target lead to discrete values for $P_{R \|}$ corresponding exactly to the longitudinal momentum change of the projectile. Thus, the measurement of $P_{R \|}$ is equivalent to projectile energy gain or loss spectroscopy. However, the recoil-ion momentum determination is almost completely independent on the quality and on the absolute velocity of the incoming beam. As a result, COLTRIMS allows energy gain measurements with typical resolutions in the order of $\Delta E_{P} / E_{P} \approx 10^{-5}$ even at $\mathrm{MeV} / \mathrm{u}$ impact velocities, whereas already the preparation of ion beams with correspondingly small momentum spread is hard to achieve. A further advantage is that projectile scattering angles in the $\mu \mathrm{rad}$ regime or even below become accessible by the determination of the recoil-ion transverse momentum without almost no limitations posed by the divergence of the incoming projectile beam. Thus, in contrast to traditional experimental methods such as electron and photon spectroscopy, COLTRIMS allows the measurement of state selective capture cross sections differential in the projectile scattering angle. The obtained data contain valuable information about the collision dynamics and the level energies of populated states.

At projectile velocities $\mathrm{v}_{P}$ which are small compared to the target electron velocity $\left(\mathrm{v}_{P}<1\right.$ a.u. $)$ it is well established that single electron capture takes place at large internuclear distances at localized crossings of the Coulomb potential curves in the incident and exit channels. This energy matching condition leads to a narrow range of finally populated projectile states. In contrast, at very high projectile velocities $\left(\mathrm{v}_{P}>>1\right.$ a.u. $)$ the matching of the electron momentum in the initial and final state determines the characteristics of the capture process which dominantly leads to transfer into low lying projectile states. Abdallah et al (1998a) used COLTRIMS to investigate the change in the energy range of populated states during the transition from low to high velocities for single and double electron capture in $\mathrm{Ar}^{16+}$ on $\mathrm{He}$ collisions at various velocities between 0.3 to $1.5 \mathrm{a} . \mathrm{u}$. With a recoil momentum resolution of 
0.2 a.u. the finally populated projectile states could be resolved with respect to their principal quantum number $n$. Figure 30 shows density plots of the longitudinal versus transverse recoil momenta for single electron capture at projectile velocities of 0.3 and 1.5 a.u., respectively. The $P_{R \|}$ values are already converted into $Q$-values using equation (4). They found, that with increasing velocity the reaction window (the range of populated states) spreads. Moreover, and in contrast to expectations based on simple models, an increasing population of higher $n$ states has been observed as the projectile velocity is raised. The authors speculated that this behavior is partly due to the increasing angular momentum transfer in collision at larger $\mathrm{v}_{P}$ enabling the population of states with both, high $n$ and angular momentum $l$, which have a high statistical weight. The projectile scattering angle for a given $Q$-value was found to be centered on the "half Coulomb angle" $\theta_{C}=Q /\left(2 E_{P}\right)$ for the lowest velocity ( $E_{P}$ : projectile energy). The angle $\theta_{C}$ separates regions of capture on the way in, leading to small scattering angles, from those on the way out giving rise to larger deflections. Thus, this indicates an about equal probability for transfer on the way in and out. However, at 1.5 a.u. velocity the projectile angular distributions are shifted to significantly larger angles than $\theta_{C}$ (Figure 30).

With an improved apparatus Fischer et al (2002) demonstrated that COLTRIMS can be used to obtain spectroscopic information about energy levels in highly charged ions which are not directly accessible by other methods. They measured state resolved differential cross sections for single electron capture from $\mathrm{He}$ in collisions with $3.15 \mathrm{keV} / \mathrm{u} \mathrm{Ne}^{7+}$ ions extracted from an EBIT (Electron Beam Ion Trap). In contrast to previous measurements and in order to obtain a very high momentum resolution the recoil ions were extracted in the longitudinal direction (i.e. along the ion beam) and deflected with a pulsed kicker onto the recoil detector placed beneath the incoming projectile beam. The finally obtained recoil momentum resolution of 0.07 a.u., which was limited only by the residual thermal spread of the gas-jet target, resulted in a $Q$-value resolution of $0.7 \mathrm{eV}$, which is sufficiently high to obtain spectroscopic information about the principal quantum number, subshell level and spin state of the captured electron. The recoil-ion $P_{R \|}$ distribution converted into $Q$-values and level energies of $\mathrm{Ne}^{6+}$ ions following single electron capture from $\mathrm{He}$ at $\mathrm{v}_{P}=0.35$ a.u. is shown in Figure 31 . The spectrum consists of three groups of lines corresponding to the $2 s 4 l$, the $2 s 3 l$ and the doubly excited states $2 p 3 l$ of Be-like Ne-ions. A line-fitting procedure has been applied after calibration of the $Q$-value axis resulting in accurate values for in total 22 level energies in the range from $121 \mathrm{eV}$ and $166 \mathrm{eV}$ with an absolute precision between $3 \mathrm{meV}$ and $100 \mathrm{meV}$. The experimen- 
tal level energies are in excellent agreement with calculated ones, except for the $2 p 3 p{ }^{1,3} S$ and the $2 p 3 s{ }^{1} P$ states, where the theoretical values deviate among each other and from the measured result by more than $0.5 \mathrm{eV}$.

During the last two years a new generation of spectrometers has been developed taking benefit from laser cooling techniques for the target preparation mainly because of two reasons. First, to extend the range of atomic targets accessible with COLTRIMS and, second, to further increase the recoil momentum resolution. Target atoms trapped and cooled in a MOT have been used to study ion-atom collisions at keV energies. Turkstra et al (2001) applied this MOTRIMS technique to study multi-electron capture processes in collisions of 3 $\mathrm{keV} / \mathrm{u} \mathrm{O}^{6+}$ with $\mathrm{Na}$ atoms. Van der Poel et al (2001) measured angle differential electron transfer cross sections in collisions of $\mathrm{keV} \mathrm{Li}^{+}$ions with laser cooled $\mathrm{Na}$ atoms. As shown in Figure 32 they obtained Fraunhofer-type diffraction patterns for the scattered projectile angular distributions in agreement with semi-classical impact parameter models. Lee et al (2002) reported on state selective capture measurements for $6 \mathrm{keV} \mathrm{Cs}^{+}$colliding with rubidium in $5 s$ and $5 p$ states, respectively, with a so far unprecedented recoil resolution of 0.03 a.u. (or a recoil velocity resolution well below $1 \mathrm{~m} / \mathrm{s}$ ). For the future, it can be anticipated that MOTRIMS will become an important tool, complementary to traditional methods, for precision spectroscopy of highly charged ions.

Reactions where two electrons are transferred from the target to the projectile contribute significantly to the total capture cross section at low projectile velocities. The ratio of double to single electron capture is about $20 \%$ for $\mathrm{Ne}^{10+}-\mathrm{He}$ collisions at impact energies between 50 and $150 \mathrm{keV}$ (Flechard et al 2001a). Usually, the capture of two electrons by slow highly charged ions leads to the population of doubly excited states in the projectile. They can decay either radiatively resulting in true double capture (TDC) or through autoionisation processes leading to a projectile which has kept only one electron and a doubly charged recoil ion (transfer ionisation (TI) or autoionising double capture). Flechard et al (2001a) measured the corresponding $Q$-value distributions for double capture from $\mathrm{He}$ in collisions with $\mathrm{Ne}^{10+}$ at impact energies of 50 and $150 \mathrm{keV}$, respectively (Figure 33) to gain information about the underlying capture mechanisms, which are still under discussion. The two electrons can be transferred either sequentially through two single capture channels or in one step through a correlated two-electron transition as proposed by Stolterfoht et al (1986) and others. Based on the analysis of state selective angular distributions and the good agreement with semiclassical coupled-channel calculations Flechard et al (2001a) concluded, that double capture in $\mathrm{Ne}^{10+}-\mathrm{He}$ collisions proceeds dominantly in two steps by independent electron interactions 
and not by processes involving the electron-electron interaction. The importance of two-step processes in populating doubly excited states has already been mentioned earlier by Abdallah et al (1998a) for $\mathrm{Ar}^{16+}-\mathrm{He}$ collisions.

The autoionising channel in multiple-electron capture collisions of slow ions with manyelectron atoms has been studied further by means of coincident Auger-electron COLTRIM spectroscopy by Hasan et al (1999). Here, the recoil momentum delivers information about the collision dynamics and the binding energies (i.e. the $Q$-value) of populated states while the coincident Auger electron spectra contain information about the subsequent relaxation pathways of highly excited projectile states. For slow $\mathrm{N}^{7+}(28 \mathrm{keV})$ on $\mathrm{Ar}$ impact it was found that double electron capture is accompanied by simultaneous excitation of the target atom with high probability (between 40 to $60 \%$ ) in overall agreement with the classical overbarrier model (see e.g. Niehaus 1986).

Recently, ion-molecule collisions and the dynamics of molecular fragmentation has been studied (Adoui et al 1999, Adoui et al 2001, Frémont et al 2002, Wolff et al 2002). At low velocities one or several electrons are captured by the highly-charged projectile and then, in a second step, fragmentation of the molecular ion occurs which is imaged using recoil-ion momentum spectroscopy. For the fragmentation of $\mathrm{CO}^{2+}$ as a consequence of electron capture reactions with $\mathrm{keV} \mathrm{O}^{7+}$ projectiles Tarisien et al (2000) were able to distinguish different fragmentation channels by means of measured kinetic energies released during the Coulomb dissociation. For molecules aligned along the beam direction they found kinetic energy releases shifted systematically to larger values which, in agreement with theoretical predictions (Wood and Olson 1999), is due to the interaction between the molecular fragments and the outgoing projectile. Fragmentation of $\mathrm{D}_{2}$ following double electron capture in collisions with $\mathrm{Xe}^{26+}$ projectiles at low velocities $(0.2-9.5 \mathrm{keV} / \mathrm{u})$ has been studied in a kinematically complete experiment by DuBois et al (2000) and Ali et al (2001). At the lowest velocity the projectile remains in the vicinity of the molecule during fragmentation leading to a transfer of internal energy from the molecule to the projectile. The fragments were found to be less energetic in the center-of-mass system of the molecule. Hence, the separation of the reaction into two steps, electron capture and subsequent fragmentation, breaks down for very slow collisions. The experimental results are in good agreement with five-body classical trajectory Monte Carlo calculations (Wood and Olson 1999, Feeler et al 1999).

The electron capture cross sections are rapidly decreasing as the projectile velocity is increased (for $\mathrm{v}_{P} \gg>1$ a.u.) while the capture mechanisms differ significantly from those at low velocities. The first kinematically complete experiment on electron capture accompanied with 
ionisation of another target electron (transfer ionisation TI) in $0.5-1.4 \mathrm{MeV}$ proton on $\mathrm{He}$ collisions has been performed by Mergel et al (1997), where different mechanisms contributing to TI could be separated. In this case three particles are in the final continuum state (corresponding to nine momentum components) demanding the determination of five momentum components to obtain the complete information. This has been achieved by measuring the recoil-ion momentum vector in coincidence with the transverse scattering of the projectile. Two reaction mechanisms could be distinguished according to their different kinematical signatures. Kinematical capture, where one electron is captured as a result of velocity matching between initial state and projectile velocity, leads to a backward emission of the recoil ion because it has to compensate the electron forward momentum. The second target electron is ionised independently in a second step due to e.g. shake-off. In contrast, in electron capture based on the e-e Thomas mechanism (Thomas 1927) the recoil-ion acts as a spectator and remains basically at rest. There, one electron is knocked by the projectile to an angle of $45^{\circ}$ and then, in a second step, it may bounce off the other target electron into the forward direction with a velocity equal to that of the projectile such that it can be captured easily. In this case the other electron is emitted perpendicular to the beam with a velocity $\mathrm{v}_{\mathrm{p}}$. The e-e Thomas mechanism leads to a scattering angle of $0.55 \mathrm{mrad}$ for the proton. Figure 34 shows the longitudinal momentum distribution of $\mathrm{He}^{2+}$ ions for different proton energies and scattering angles. The left vertical lines show the expected values of $P_{R \|}$ for kinematical capture at the given projectile energies. The full curves are results of a calculation where the two-electron transition is treated as two independent one-electron transitions for transfer and ionisation. Thus, the e-e Thomas mechanism is not taken into account in this independent electron approximation. The appearance of an additional structure in the recoil-ion momentum distribution centered around $P_{R \|}=0$ for $0.55 \mathrm{mrad}$ scattering angles has been interpreted as a clear signature for capture due to the e-e Thomas mechanism.

In a later publication Mergel et al (2001) and Schmidt-Böcking et al (2002; 2003; 2003a) analyzed the electron emission characteristics for the same collision system at small scattering angles. In this case, transfer ionisation is expected to be well described as a two-step process. One electron is captured due to kinematical capture and the second electron is ionised due to either a shake-off process or due to ionisation by an independent encounter with the proton. The slow ionised electron is expected to be emitted either isotropically or preferentially in the transverse direction. However, most of the electrons were found to be emitted into the backward direction in a narrow cone around the $\mathrm{H}^{0}$ scattering plane. This is surprising, because for two independent scattering events the transverse momentum transfer would be randomly ori- 
ented with respect to the scattering plane. Moreover, the measured transverse momenta of both, the electron and the recoil ion, never coincided with the locations expected for a twostep process. From these observations the authors concluded that the correlated electron momenta in the initial He ground state play a crucial role. The capture process picks out, by velocity matching, components of the initial state wave function for which one electron has a large forward directed momentum equal to $\mathrm{v}_{P}$. In a correlated initial state, however, a large forward momentum of one electron might correspond to a high backward momentum of the second electron, which appears in the continuum after ionisation.

Recently, Schmidt et al (2002) succeeded to apply COLTRIMS for the study of electron capture and transfer ionisation in $2.5-4.5 \mathrm{MeV}$ p-He collisions. Using an intense beam of the ion storage and cooler ring CRYRING and a novel switching technique for the recoil spectrometer with an electrically gated recoil-ion drift path they were able to measure cross sections as small as $10^{-26} \mathrm{~cm}^{2}$. A close connection between TI and photoionisation has been found. The probability for electron emission in kinematical capture decreases with increasing velocity and approaches the photoionisation shake-off value of $1.63 \%$. In both cases (TI and photoionisation) very little momentum is transferred to the first removed electron, which leaves the target with a velocity determined by the projectile, while the second electron is ionised via shake-off. In the same experiment the high-velocity dependency of the e-e Thomas cross section has been measured which is found to be in agreement with the theoretically predicted $\mathrm{v}_{\mathrm{P}}^{-11}$ - scaling.

\subsubsection{Projectile-Ionisation: A Novel Approach to (e,2e)-Experiments on Ions}

The ionisation of a non-bare projectile in an ion-atom collision may proceed via an interaction of the projectile electron with an electron $(e, e)$ or the nucleus $(e, n)$ of the target as illustrated in Figure 35. Interest arises on the one hand since projectile ionisation represents the cleanest situation to study dynamical electron-electron correlation in a challenging, still fundamental four-body process involving two heavy and two light particles. On the other hand, a strict experimental separation of both contributions to projectile ionisation might pave the way to perform differential experiments on the ionisation of ions by electron impact, one of the most fundamental processes in atomic collision physics.

The importance of effective electron-ion collisions in energetic ion-atom collisions has been pointed out very early by Bates and Griffing $(1953,1954,1955)$. Driven by the motivation mentioned above, numerous theoretical (see e.g. McGuire et al (1981); Hippler et al (1987); 
Lee et al (1992); Fiol et al (2001)) as well as experimental studies have been reported in the literature. A first experimental identification of the (e,e) contribution by Montenegro et al (1992) exploited its threshold behavior by measuring the velocity dependence of total projectile ionisation cross sections. Further investigations concentrated to identify this contribution by the appearance of characteristic transition lines in high-resolution zero-degree electron spectra (Zouros 1996) or by its specific kinematical signatures (Montenegro et al 1993). Calculations by Montenegro and Meyerhof (1992) indicated that the (e,e) interaction dominates the cross section for projectile ionisation at large inter-nuclear distances $b$ since the nuclear potential of the target, that might cause ionisation of the projectile in an $(\mathrm{n}, \mathrm{e})$ interaction as well, is effectively screened by the target electrons as illustrated in Figure 35. In addition, the (e,e) contribution ("anti-screening") leaves the target nucleus as a spectator without any significant final momentum, whereas it noticeably recoils if the screened target nuclear potential takes over the active part in the (n,e) reaction at smaller $b$ ("screening").

Substantial progress was achieved about ten years ago using recoil-ion momentum spectroscopy. Measuring the target (recoil)-ion momentum distribution, two maxima in the doubly differential cross sections a function of recoil-ion momentum were identified by Dörner et al (1994) and Wu et al (1994). Their projectile velocity dependent relative locations were closely related to those expected from target-ion kinematics for each of the processes and essentially reproduced by n-electron classical trajectory Monte Carlo calculations (nCTMC) with two active electrons. Surprisingly, close to threshold, where the two maxima were observed, the calculations were only found to be in quantitative agreement with the experiments if an additional reaction channel, double target ionisation plus electron exchange, was taken into account.

Very recently, the first kinematically complete measurement has been reported on simultaneous single ionisation of the projectile and of the target in $\mathrm{C}^{2+}$ on He collisions at 3.6 $\mathrm{MeV} / \mathrm{u}$ by Kollmus et al (2002). Projectile ionisation was identified by separating the emerging $\mathrm{C}^{3+}$ ions in a magnet and detecting them with a fast scintillation counter. In a Reaction Microscope, the vector momenta of all other collision fragments have been detected in coincidence with the emerging $\mathrm{C}^{3+}$ ions covering the major part of the twelve-dimensional 4-particle final-state momentum space.

As is pointed out by Kollmus et al (2002) and illustrated in the lower part of Figure 35, (e,e) and $(n, e)$ contributions to projectile ionisation are expected to differ in the correlated dynamics of the active particles: Whereas the active target electron should dominantly compensate the major part of the momentum transfer $\vec{q}$ to the $\mathrm{C}^{2+}$ projectile in an (e,e) ionisation event, 
this role is taken by the target nucleus if the (n,e) interaction dominates. Since $\vec{q}=-\left(\vec{P}_{R}+\vec{P}_{e}\right)$ with $\vec{P}_{e}$ being the momentum of the emitted target electron, the criterion that $\left|\vec{P}_{R}\right| \geq\left|\vec{P}_{e}\right|$ or vice versa was used to identify the active role of one or the other emerging target fragment (the upper index " $\mathrm{f}$ " for final is omitted for convenience). As illustrated in the lower part of Figure 35 , the particular fragment taking over the active role in a certain collision is expected to be scattered predominantly opposite to the ionised projectile electron in the azimuthal plane.

In Figure 36 measured as well as calculated angles $\Phi(e, e)$ are plotted versus $\Phi(n, e)$ for all events (upper row), for events with $\left|\vec{P}_{e}\right| \geq\left|\vec{P}_{R}\right|$ where the (e,e)-process is be expected to dominate (middle row) and for $\left|\vec{P}_{e}\right| \leq\left|\vec{P}_{R}\right|$ (lower row) where the target nucleus might take over the active part. Even without any condition (left upper frame), a significant pattern was observed. Moreover, it was found that a major part of all events was divided into two clearly separated regimes where either $\Phi(e, e)$ or $\Phi(n, e)$ is close to $180^{\circ}$ for $\left|\vec{P}_{e}\right| \geq\left|\vec{P}_{R}\right|$ (left column, middle) or $\left|\vec{P}_{e}\right| \leq\left|\vec{P}_{R}\right|$ (left column, bottom), respectively. These two regimes can be uniquely related to collisions where either the $(e, e)$ or the $(n, e)$ process dominates the projectile ionisation. The experimental results (left column) were observed to be in excellent agreement with theoretical predictions of six-body CTMC calculations that include the two nuclei, the He electrons and the L-shell electrons on $\mathrm{C}^{2+}$ (middle column). Even details observed in the experimental data, like systematic variations of the mean value for $\Phi(n, e)$ and $\Phi(e, e)$ around $180^{\circ}$ or the $\Phi(n, e)$-dependent variation in intensity for the (e,e)-events were found to be reproduced by theory.

The latter features clearly demonstrate that three- or four-body interactions are still important. In order to investigate whether this influence can be reduced, another Monte Carlo calculation has been performed for more asymmetric initial conditions, namely for $3.6 \mathrm{MeV} / \mathrm{u} \mathrm{C}^{2+}$ on $\mathrm{H}$ collisions with the active target electron in an excited $n=2$ state (right row). Now, the importance of the (n,e)-reaction was found to be drastically reduced, the $\Phi(e, e)$ angular distribution is always exactly peaked at $180^{\circ}$ independent of $\Phi(n, e)$ and the recoiling-target ion is isotropically scattered with respect to the projectile electron. The latter two indicate that the recoil-ion now has perfectly taken over the role of a spectator (see also Olson et al (2003)). 
In a further step, the authors were able to demonstrate that the subset of events which fulfilled the condition $\left|\vec{P}_{e}\right| \geq\left|\vec{P}_{R}\right|$ closely reflected the dynamics expected for the ionisation of $\mathrm{C}^{2+}$ by 2 $\mathrm{keV}$ electron impact, i.e. for the kinematically inverted electron-ion collision system. This is illustrated in Figure 37, where the scaled momentum transfer $\left|\vec{q}^{*}\right|=q \cdot\left(2 \cdot I_{e_{-} b}\right)^{-1 / 2}$ is plotted versus the emission angle $\vartheta_{e}$ of the $\mathrm{C}^{2+}$ electron with respect to the momentum transfer direction for electron-impact ionisation of $\mathrm{C}^{2+}$ (upper part) and for $2 \mathrm{keV}$ electron on $\mathrm{He}$ collisions (lower part) in a co-planar geometry. The momentum transfer has been scaled to take into account the different ionisation potentials $I_{e_{-} b}$ in both systems. In both panels, the major part of the electrons is seen to be emitted along $\vec{q}$ in binary collisions with the projectile electron, forming the well-known "binary peak". Around $180^{\circ}$ and for small momentum transfers, another characteristic structure becomes visible, the so-called "recoilpeak". Here, the electron is found to be emitted into the $-\vec{q}$-direction due to its interaction with the recoiling target nucleus balancing both the momentum transfer and the ejected electron momentum (for electron impact see also Figure 23 in Section 4.3).

In summary, exploiting the full capabilities of Reaction Microccopes in a quadruple coincidence, it was finally demonstrated, nearly 50 years after its first prediction, that one is indeed able to clearly isolate all collision events where the (e,e) reaction dominantly contributed. It was further shown that these events essentially display all features usually observed in $(e, 2 e)$ electron-atom collision experiments paving the way to future $(e, 2 e)$ investigations for all ions over a large velocity regime in heavy-ion storage rings. 


\subsubsection{Ionisation by Slow Projectiles: Saddle Point Electrons}

At small projectile velocities, where ionisation can not be treated perturbatively, the production of free electrons has to be explained by other mechanisms. In classical-trajectoryMonte Carlo (CTMC) calculations (Olson et al 1997) for $\mathrm{p}+\mathrm{H}$ collisions Olson found electrons emitted in forward direction with nearly half of the projectile velocity. Olson (1983, 1986) and Olson et al (1987) assumed that these electrons are ones that are left stranded equidistant between the projectile and target nucleus ions and are balanced in place by the attractive Coulomb forces of both ions. In quantum mechanical calculations done by Winter and Lin (1984) the relevance of the saddle point (SP) of the two center Coulomb potential was demonstrated. These authors used a triple-center atomic-state method with one center located at the SP.

The velocity of the SP depends on both, the charges of the projectile as well as of the target nucleus. In the late $80 \mathrm{~s}$ several measurements at collision energies between 50 and $100 \mathrm{keV} / \mathrm{u}$ done at Rolla (Irby et al 1988, Gay et al 1990) and Bariloche (Bernardi et al 1989, 1990) searched for that dependency to demonstrate the existence of the SP mechanism. These measurements at only slightly different acceptance angles performed with dispersive electron spectrometers yielded conflicting results. Furthermore, it was controversial which one of the two differential cross sections, $d \sigma /(d v d \Omega)$ or $d \sigma / d v$, has to be used to observe a peak at the SP velocity (Meckbach et al 1991). Only the Rolla group claimed evidence for the SP mechanism from their experimental data.

Theoretical progress was made by investigating the electron trajectories in CTMC calculations during the collision (Bandarage and Parson 1990, Illescas et al 1998). For a more precise classification it was suggested to call only those electrons SP electrons who reach a positive electronic energy slowly, at large internuclear distances R. In contrast, the competing direct impact (DI) ionisation causes an abrupt transfer to the continuum. Sidky et al (2000) found that the electrons, which already have positive energy at small $\mathrm{R}$, are also preferentially emitted with the SP velocity and concluded that no experimental findings at all are qualified to substantiate the SP mechanism. To avoid all of this controversy we here use the term "saddle point electrons" not in the sence of the "SP process" but referring to the location in momentum space close to the SP.

In quantum mechanical calculations within the semi-classical approximation using adiabatic molecular eigenstates the ionisation has to be described by an infinite series of transitions between these states. At complex R the states of the same symmetry are connected by branch points. The Hidden Crossing theory (Solov'ev 1986, Pieksma and Ovchinnikov 1991) deter- 
mines the ionisation probability for $\mathrm{p}+\mathrm{H}$ collisions by integration in the plane of complex $\mathrm{R}$. Different pathways circumventing the branch points had been found. The so called S promotion occurs at small $\mathrm{R}$ and can be related to the DI ionisation, while the $\mathrm{T}$ series branch points involve increasing $\mathrm{R}$ and are associated with electrons located at the SP. The T series starts out with $\sigma$ as well as $\pi$ states. The $\pi$ states are populated by rotational coupling from the $\sigma$ initial states. Both molecular symmetries result in a broad maximum in the longitudinal electron momentum distribution centered at half of the projectile velocity. The $\pi$ states, however, cause a nodel-line for electron emission along the projectile axis which includes the SP (Pieksma and Ovchinnikov 1994, Ovchinnikov and Macek 1995).

Because of the nodel-line structure spectrometers with small acceptance angle are not qualified to explore $\pi$-state dominated SP emission. Therefore, Pieksma et al (1994) used a TOF-spectrometer with large acceptance angle to measure the longitudinal electron momentum distribution at 1-6 keV p $+\mathrm{H}$ collisions. They found agreement with the theoretical results (Pieksma and Ovchinnikov 1991) but did not resolve the transversal momentum. First two-dimensional momentum distributions of SP electrons were measured by Kravis et al (1996) and Abdallah et al (1997) by projecting the electrons and recoil ions with a high electric field onto position sensitive detectors. Due to the large extraction fields used in the experiment in order to efficiently collect electrons, the recoil-ion momentum and, thus, the nuclear scattering plane was not resolved and a $\pi$-state dominated structure could not be observed.

For slow collisions the momentum exchange between the projectile and target nucleus is typically much larger than the emitted electron momentum (see e.g. Dörner et al (1997)). Thus, the nuclear motion in the semi-classical approximation proceeds in a plane ("nuclear scattering plane"). As a result of the symmetry of the system any $\pi$ states populated from $\sigma$ initial states are lying in this plane. The first experiment which was able to determine the scattering plane by using COLTRIMS in addition with slow electron detection was reported by Dörner et al (1996a). For 5-15 keV p + He collision they observed the electron momentum distribution projected onto the scattering plane. Most electrons were found in two jets in forward direction with a minimum at the saddle point in between (see Figure 38a). This node on the SP is expected for $\pi$ states. The relative weight of the two jets changes with the collision energy. This was interpreted as a coherent superposition of $\pi$ and $\sigma$ contributions with a phase depending on the projectile velocity. Macek and Ovchinnikov (1998) pointed out that these experimental data can be used to extract information about the quasi-molecule formed during the collision, e.g. the potential energy curves of the states coupled to the continuum. 
Further experiments using COLTRIMS have been reported by Abdallah et al (1998, 1998b, 1999) using $\mathrm{He}^{+}, \mathrm{He}^{2+}$ and $\mathrm{Ne}^{+}$as projectile and $\mathrm{He}$ and $\mathrm{Ne}$ as targets. While the two-finger structure found for the $\mathrm{p}+\mathrm{He}$ system was also observed for $\mathrm{He}^{+}$and $\mathrm{He}^{2+}$ projectiles, in $\mathrm{Ne}^{+}+$ Ne collision two spiral arms were emanating from the target and the projectile "position" into opposite directions (see Figure $38 \mathrm{~b}$ ).

None of these experiments has measured the full electron momentum vector. The projection onto two-dimensional planes always contained the beam axis while the $\pi$ characteristic can be visualized best in the plane perpendicular to the beam axis. Such a distribution has been measured by Schmidt (2000). He studied the transfer ionisation in $\mathrm{He}^{2+}+\mathrm{He}$ collisions, where one of the target electrons is captured to the projectile. In this perspective the dipole pattern of the $\pi$ states can clearly be seen lying in the scattering plane (see Figure 38c). For transfer ionisation in $\mathrm{He}^{2+}+\mathrm{He}$ as well as $\mathrm{He}^{2+}+\mathrm{H}_{2}$ collisions Afaneh et al (2002) extended the acquired range of longitudinal electron velocity and observed a noticeable fraction of electrons moving about 1.2 times faster than the projectile velocity (see Figure $38 \mathrm{~d}$ ).

The tremendous experimental progress motivated further semi-classical calculations. Sidky and Lin $(1998,1999)$ calculated electron distributions for $\mathrm{p}+\mathrm{H}$ collisions using the twocenter momentum space discretization (TCMSD). In comparison with the experimental data for $\mathrm{p}+\mathrm{He}$ (Dörner et al 1996a) only a qualitative agreement was found. For $\mathrm{p}+\mathrm{He}$ collisions at higher impact energy up to $100 \mathrm{keV}$ Edgü-Fry et al (2002) compared experimental results to TCMSD calculations, which also treat only one active electron but at an adapted target potential. Only for the longitudinal electron velocity distributions reasonable agreement was obtained. For the $\mathrm{p}+\mathrm{H}$ system further theoretical work solving the time dependent electronic Schrödinger equation on a Cartesian grid in configuration space was reoerted by Schultz et al (2002). Chassid and Horbatsch (2002) used the Fourier collocation method, which switches between grids based on coordinate or momentum space representation during the time propagation. These most recent theoretical results on atomic hydrogen cannot be compared with experimental momentum space images because the COLTRIMS method requires an internally cold gas target, which is not available for atomic hydrogen.

While impressive theoretical progress was achieved in the treatment of electron transfer reactions in slow collision even for highly excited states of multi-electron systems (see e.g. Fritsch (1994)) the understanding of the ionisation processes at slow projectile velocities is far from being satisfying. All calculations for such collisions performed so far are effective oneelectron approximations treating either the $\mathrm{p}+\mathrm{H}$ collision system or simulate the He target by an effective potential. The fully differential experimental results available today for multi- 
electron systems have only been understood qualitatively and, therefore, provide a major challenge for the future.

\subsubsection{Ionisation by fast Projectiles: Attosecond Pulses}

This Section summarizes a wealth of new results on single and multiple ionisation of atoms by fast ionic projectiles. Due to the large number of very recent data, we concentrate to exclusively report on those that have been obtained after the last review. Readers interested in total cross sections, single or double differential data (Cocke and Olson 1991; Ullrich 1994), in electron emission cross sections (Stolterfoht et al 1994), in previous reviews (Dörner et al 2000; Ullrich et al 1994; 1997) or in a comparison with ionisation by strong laser pulses (Ullrich and Voitkiv 2002) have to be referred to the cited literature.

Subsection 4.4.4.1 concentrates on describing recent results obtained for single ionisation at small and large perturbations. In subsection 4.4.4.2, new double ionisation data are presented followed by a short discussion on multiple ionisation processes in section 4.4.4.3. 


\subsubsection{Single ionisation at small and large perturbations}

Single ionisation at small perturbations $Z_{P} / v_{P}<1\left(Z_{P}, v_{P}\right.$ : projectile charge and velocity, respectively) was explored in great detail over more than three decades for electron impact in kinematically complete investigations (see Section 4.3; for recent reviews see McCarthy and Weigold (1991); Lahmann-Bennani (1991); Coplan et al (1994)). Here, the first Bornapproximation (FBA) has been successfully used to theoretically describe the experimental data, mostly measured in "co-planar" geometry where the ionised target electron is detected in the scattering plane of the fast electron. In the FBA, all cross sections scale with $Z_{P}^{2}$. Hence, no differences are expected for positively charged ion impact, where kinematically complete experiments only became feasible since 1994 (Moshammer et al 1994) and fully differential cross sections (FDCS) have not been reported before 2001 (Schulz et al $(2001 ; 2002)$ for unpublished data see Weber 1998).

In Figure 39, the main characteristics of the three-particle momentum balance at small perturbations $\left(Z_{P} / v_{P}=0.06\right)$ are illustrated for single ionisation of $\mathrm{He}$ for $6 \mathrm{MeV}$ proton impact. Here, the final state momenta of the electron, the recoiling $\mathrm{He}^{1+}$ target ion, as well as the momentum change of the scattered projectile are shown (see also Moshammer et al (1997) for $1 \mathrm{GeV} / \mathrm{u} \mathrm{U}^{92+}$ impact). Exploiting azimuthal symmetry, all momenta are projected onto a plane defined by the incoming projectile momentum vector $\vec{P}_{P}^{i}=P_{P \|}$ and the momentum vector of the recoiling ion $\vec{P}_{R}=\left(-P_{R x}, P_{R \|}\right)$. The momentum change of the projectile in the longitudinal direction is small $q_{\|}=\Delta E_{P} / v_{P} \leq 0.4 a . u$. for typical electron energies $E_{e}<200 \mathrm{eV}\left(\Delta E_{P} \approx E_{e}+E_{e_{-} b}:\right.$ projectile energy loss). The experimental $\Delta P_{P \|}$-resolution in Figure 39 was about 0.1 a.u. Thus, essentially "no" momentum is transferred to the target in the longitudinal direction and the transverse momentum transfer is found to be mainly directed opposite to the recoiling target ion, indicating the impoartance of nuclear scattering.

Deeper insight into the collision dynamics has been obtained by investigating FDCSs as illustrated in Figure 40 for single ionisation of helium in collisions with $100 \mathrm{MeV} / \mathrm{u} \mathrm{C}^{6+}$ projectiles at $Z_{P} / v_{P}=0.1$ (Schulz et al 2001; 2002; 2003; Madison et al 2002). Here, the FBA is assumed to perfectly describe the data. Shown are experimental (Figure 40a) and theoretical (Figure 40b) complete three-dimensional (3D) emission patterns for target electrons with defined energy $\left(E_{e}<6.5 \mathrm{eV}\right)$ at a fixed momentum transfer $\vec{q}$ with $|\vec{q}|=0.75$ a.u. as a function of the azimuthal $\left(\varphi_{e}\right)$ and polar $\left(\vartheta_{e}\right)$ electron emission angles, respectively. The initial pro- 
jectile momentum $\vec{P}_{P}^{i}$ directed along the z-axis in Figure 39, now points upwards for better 3D illustration of the emission pattern.

The electron emission in 3D exhibits a characteristic double peak structure with two maxima. One is along the momentum transfer direction and the second opposite; the well-known "binary" and "recoil" peaks, respectively (for their interpretation see the detailed description in Subsection 4.3 and e.g. Stefani et al (1990), Whelan et al (1993)). The theoretical results, which include higher-order contributions in the interaction of the projectile with the target beyond the FBA (for details see Madison et al (2002)) were found to be nearly cylindrically symmetric around the $\vec{q}$-axis, a feature characteristic for any first order approach. The sharp 3D minimum at the origin indicates both, the absence of higher-order processes as well as the dominance of dipole transitions. Whereas the experimental data were observed to be in close agreement with the predictions in coplanar geometry, i.e. for a cut of the three-dimensional pattern along the plane defined by $\varphi_{e}=0^{\circ}$, dramatic deviations were observed out-of-plane. Here, the experimental FDCS do not shrink to zero close to the origin but exhibit a distinct structure with two maxima in their $\vartheta_{e}$-distribution (counted within the plane tilted by $\varphi_{e}$ ) at $\vartheta_{e}=90^{\circ}$ and $270^{\circ}$, respectively. In the plane perpendicular to the scattering plane and containing the initial momentum $\vec{P}_{P}^{i}$, i.e. for $\varphi_{e}=90^{\circ}$ the two maxima are of equal size, whereas theory predicts an angular independent, constant behavior.

Two potential reasons have been discussed in the literature for these surprising features in out-of-plane geometry at low perturbations, where the applicability of the FBA was taken for granted on the basis of electron impact FDCSs (nearly exclusively investigated in co-planar geometry). First, in a kind of two-step higher-order process the projectile might be deflected by interacting with the ionised target electron in one step (Schulz et al 2003). In another step, the projectile elastically scatters off the residual target ion resulting essentially in a rotation of the scattering plane around the azimuthal $\varphi_{e}$ angle. Obviously, the details of the projectile deflection then sensitively depend on the relative distance between the passing projectile with respect to the negative electron-charge distribution on the one hand and to the nucleus on the other hand, i.e. on the wave function. This leads to the second possible explanation put forward by Madison et al (2003), namely that the scattering wave function used in Schulz et al 2003 might not be good enough at small distances relative to the nucleus.

Nuclear scattering in single ionisation has been well known to strongly modify differential cross sections at large projectile deflection angles (see DeHaven et al (1998) for a recent ex- 
periment, discussions in Dörner et al (2000); Ullrich et al (1997) and references therein). Its influence at very small momentum transfers, however, came as a surprise demonstrating that three-dimensional imaging of the ejected electrons, i.e. taking 3D pictures of the final state wave-function square, reveals new insight into the fundamental dynamical three-body problem. Whether or not similar features do occur in electron collisions in out-of-plane geometry is not clear by now but experiments are underway. Further kinematically complete data have been recorded by Weber (1998) (Figures 31 and 32 in Dörner et al (2000)) for $0.5 \mathrm{MeV}$ proton on He collisions and FDCSs can be found in Weber (1998).

Relativistic effects for impact ionisation of light target atoms (see e.g. Voitkiv 1996; Voitkiv et al 1999) have never been explored in kinematically complete experiments, since traditional techniques rely on the direct determination of the projectile deflection angle and final energy which is not possible at relativistic velocities. With the advent of Reaction Microccopes such studies have become feasible for the first time, since here the momenta of the recoiling target ion $\vec{P}_{R}$ and of the emitted target electron $\vec{P}_{e}$ are measured instead, obtaining $\vec{q}$ from momentum conservation $\vec{q}=\vec{P}_{P}^{i}-\vec{P}_{P}^{f}=\left(\vec{P}_{R}^{f}+\vec{P}_{e}^{f}\right)$ which, hence, is accessible for any projectile velocity with the same resolution of presently about 0.1 a.u..

Relativistic effects have first been discussed within the context of "classical trajectory Monte Carlo calculations" (CTMC) (Wood et al 1997) in order to explain experimental data for 1 $\mathrm{GeV} / \mathrm{u} \mathrm{U}^{92+}$ on helium collisions at a relativistic factor $\gamma=\left(1-v_{P} / c\right)^{1 / 2}=2$ (Moshammer et al 1997). It was found that the post collision interaction (PCI), a higher-order effect that strongly influences the ionisation dynamics (see next paragraph), was considerably suppressed due to the relativistic compression of the projectile field along the propagation direction. More recently, relativistic effects and deviations from the dipole approximation (non-relativistic photon-limit) have been discussed for the same collision system (Voitkiv et al 2002; 2002a; 2003). In Figure 41 the doubly differential cross section for electron emission is plotted as a function of the longitudinal electron momentum, integrated over certain transverse electron momenta. Clear differences are observed between the dipoleapproximation (dotted line), the non-relativistic FBA (dashed line) and the relativistic first Born results (full line) being in significantly better agreement with the experiment.

The relationship between single ionisation by fast charged particles and photo-ionisation, that has been intensively discussed in the past (see e.g. Bethe (1930); Moshammer et al (1997); Stolterfoht et al (1999), Ullrich et al (2000); Dorn et al (2001); Ullrich and Voitkiv (2002) and references therein), has been theoretically explored recently for the first time in terms of 
FDCSs. The fully relativistic treatment (Voitkiv and Ullrich 2001) lead to the "important conceptual result, that collisions with minimum momentum transfer, which are often termed the optical limit and are regarded to be closest to photo-absorption (Rudd et al 1992), in fact can never be photon-like". Whereas the oscillator strength is the same, directions are just orthogonal with the dipole pattern oriented perpendicular to the photon propagation for photoabsorption and along the projectile direction for charged particle impact. Instead, as has been shown by Voitkiv and Ullrich (2001) the interaction with the charged-particle induced field indeed becomes photon-like, meaning that the absorption of so-called "transverse" virtual photons dominates, for small but not to small transverse momentum transfers $\vec{q}_{\|} / \gamma^{2}<<q_{\perp}<<\vec{q}_{\|}=\Delta E_{P} / v_{P}$.

Deviations from the FBA at strong perturbations $Z_{P} / v_{P} \gg 1$ have been extensively discussed in the literature for electron and ion impact (for ions see e.g. Stolterfoht et al (1994) and references therein), in the latter case mostly at large electron energies. With the advent of electron momentum spectroscopy, low-energy electrons became reliably measurable for the first time with meV resolution and higher-order effects were observed at very low energies as well in a pioneering experiment by Moshammer et al (1994) (see also references therein for previous measurements using conventional spectrometers).

As illustrated in Figure 42, single ionisation dynamics changes significantly with increasing perturbation for fast, highly-charged fast ion impact (for a detailed discussion see Moshammer et al (1994); Ullrich et al (1995); Moshammer et al (1997a); Schmidt et al (1998); Olson et al (1998)). Again this is a situation, namely large perturbations at high velocities, that is not accessible for electron impact where $\left|Z_{P}\right|=1$. While the general characteristics of the dynamics prevails, i.e. small momentum transfers by the projectile still dominate and a pronounced balancing of momenta between recoil-ion and emitted electron is observed, the final-state, however, obviously becomes more and more "deformed" with increasing perturbation strength. This effect, which was very well described by CDW approaches (see e.g. Crothers and McCann (1983); Fainstein et al (1996); Gulyás et al (2000); Fainstein et al (2001); full lines in the right-hand panels of the Figure: O'Rourke et al (1997)), has been interpreted as a "post-collision-interaction" (PCI), an interaction of the receding highly-charged projectile ion with the emitted low-energy target electrons and ions in the final state: Electrons are dragged behind the projectile whereas the recoil ion is pushed backwards with about the same force. Reversing the sign of the projectile charge should interchange the role of electrons and ions in the final state, an effect that has been investigated for antiproton impact (Khayyat et al 1999). 
Even at strong perturbation, only very little momentum is transferred to the target system as a whole into the longitudinal direction for swift collisions. Thus, the width of the momentum distribution along the projectile propagation has been interpreted as a quantity, being inherent to the target itself, namely to the momentum distribution in the ground state, the so-called Compton profile (Dörner et al 1995). The visibility of bound-state properties has been discussed by Moshammer et al (1999). In Figure 43 experimental longitudinal electron momentum distributions (DDCS) are shown for different cuts in $P_{e \perp}$ for single ionisation of argon (symbols) by $3.6 \mathrm{MeV} / \mathrm{u} \mathrm{Au}^{53+}$ impact along with theoretical CDW-EIS predictions (full line, Fainstein et al (1996), Moshammer et al (1999)) at a very strong perturbation $Z_{P} / v_{P}=4.4$. Structures were identified near $v_{e \|}= \pm 0.5 \mathrm{a} . u$. in theory within the error bars of the experimental data. In this first calculation, the enhancements were interpreted to be related to the nodal structure of the $3 \mathrm{p}_{0}$ state. Whereas this direct signature of the ground-state momentum distribution (inset) could not be verified in more recent calculations (Gulyás et al 2000) it was found, however, in agreement with Moshammer et al (1999) that different subshells lead to pronounced differences in the longitudinal electron momentum distribution and that all substates have to be considered in order to reproduce the experimental spectrum. Thus, the experiments provided strong evidence that the longitudinal electron momentum distributions reflect the properties of the respective bound-state wave functions.

Recently, multiply or even fully differential cross sections have been reported for strong perturbations as well (Schulz et al 2002; Moshammer et al 2001; Fischer et al 2003a) yielding dramatic disagreement with essentially all non-perturbative theoretical models at hand. Similar as for small perturbations, the proper treatment of the full three-body dynamics seems to represent a major challenge for theory (see also Olson and Fiol 2001). In Figure 44, DDCSs for electrons of different energies are plotted as a function of the projectile transverse momentum transfer $p_{\perp}=P_{P \perp}^{f}=\vartheta \cdot P_{P}^{i}$ or deflection angle $\vartheta$ for $100 \mathrm{MeV} / \mathrm{u} \mathrm{C} \mathrm{6}^{6+}$ (Figure 44a) and 3.6 $\mathrm{MeV} / \mathrm{u} \mathrm{Au}^{53+}$ (Figure 44b) on helium collisions i.e. for a perturbative $\left(Z_{P} / v_{P}=0.1\right)$ as well as strongly non perturbative $\left(Z_{P} / v_{P}=4.4\right)$ situation, respectively (Moshammer et al 2001). At moderate electron energies, $E_{e}>50 \mathrm{eV}$, essentially two different dynamical contributions to the spectra can be identified: First, a broad, unstructured shoulder (the only contribution for $E_{e}<50 \mathrm{eV}$ ) at momentum transfers by the projectile $q_{\perp}=-p_{\perp}$ smaller than the respective electron energy in a binary projectile-electron collision: $E_{e}<p_{\perp}^{2} / 2 m_{e}\left(m_{e}\right.$ : electron mass). These are electrons that mainly balance their momenta with the recoiling target ions, emitted in a dipole-like interaction with the projectile field without significant momentum transfer. 
Second, a pronounced shoulder or even a broad peak is observed at transverse projectile momentum transfers that match the respective electron energies $E_{e}=p_{\perp}^{2} / 2 m_{e}$, the so-called "binary encounter electrons" (BEE). Whereas the experimental results at small perturbation for fast $\mathrm{C}^{6+}$ impact were found to be reasonably well described by an FBA calculation (full line in Figure 44a), dramatic deviations between experiment and theory were observed at large perturbations. A standard CDW calculation (dashed line in Figure 44b) that does not include the interaction between the nuclei fails completely. Various improvements of theoretical approaches (Olson and Fiol 2001), now implementing the inter-nuclear interaction on different footings and, thus, trying to consider the full three-particle problem, tend to improve the situation (see for example full line in Figure 44b) but, in general, are in surprisingly poor agreement with the experiment. Classical calculations that are usually found to describe the many-body dynamics in strong ion-induced fields quite well don't find at all any binary electrons (Olson and Fiol 2001). As in the case for small perturbations, the reasons for these discrepancies are by no means clear at present.

This has been further elucidated recently by Schulz et al (2002), who investigated FDCSs for the same collision system, presented in Figure 45 for coplanar geometry. Electron emission energies are $E_{e}=17.5 \mathrm{eV}$ (top) and $E_{e}=55 \mathrm{eV}$ (bottom) at fixed momentum transfers of 0.65a.u. (Figure 45a), 1.0a.u. (b) and 1.5a.u. (c). At small $q$ (a), the binary peak predicted by theory, along the $\vec{q}$-direction for the FBA (dashed line) and shifted into the forward direction in CDW calculations (full line), is only hardly visible in the experiment, whereas a strong new peak emerges pointing exactly into the forward direction. Here, the absolute magnitude is underestimated by a factor of 36 to 200 by the standard CDW, where the interaction between the nuclei was not yet considered. Such calculations are just being developed and have not been published yet (Madison et al 2003; Fischer et al 2003a,b). Increasing the momentum transfer (Figures $45 \mathrm{~b}, \mathrm{c}$ ), the binary peak more and more dominates, yielding increasingly better agreement with theory.

In summary, the few here-mentioned topics addressing single ionisation by ion impact, namely comprehensive pictures in momentum space including out-of-plane geometries, relativistic effects at large velocities and strongly non-perturbative situations for highly charged ion impact at large velocities, demonstrate in a most impressing way the power of new projection techniques to elucidate many-particle dynamics in situations that have not been accessible with traditional methods. Surprisingly, even after 30 years of research, which, however, was strongly focussed on electron impact studies in co-planar geometry, comparison with 
state-of-the-art theoretical approaches bring to light that even the most simple dynamical situation, the effective three-body Coulomb problem, still seems to be very poorly understood in a general sense.

\subsubsection{Double ionisation at small and large perturbations}

Double ionisation by ion, photon and electron impact has been excessively discussed in the past (for a review see e.g. McGuire (1997)). For ion impact, mostly total cross sections were considered (for a review see Cocke and Olson (1991)) until recoil-ion momentum spectroscopy was developed and singly as well as multiply differential data were reported (for reviews see Dörner et al (2000); Ullrich et al (1994, 1997)). Starting with a pioneering experiment by Moshammer et al (1996), few kinematically complete experiments for ion as well as for electron impact were reported using Reaction Microccopes. Only recently experimentalists succeeded to decisively accelerate data taking, essentially by using nanosecond pulsed ion beams, such that highly or even fully differential cross sections could be projected from huge data sets (Fischer et al 2003). These comprehensive sets of FDCSs provide the ultimate benchmark for the comparison with theory and are exclusively discussed in this paragraph.

In a simplified, though often stressed illustrative picture based on a perturbation expansion, double ionisation by charged particle impact can either occur due to an independent interaction of the projectile field with both target electrons (termed "two-step-2", TS-2) or, due to a single interaction of the field with the atom, where the second electron is emitted as a result of the electron-electron correlation. In collisions with charged particles as for photoionisation the latter process usually is further subdivided in terms of many-body perturbation-theory diagrams: Two-step 1 (TS-1), a single interaction of the projectile with the target plus a second step, when the emerging first electron interacts with the second one, is distinguished from shake-off (SO) or ground-state (GS) correlation contributions (for diagrams see McGuire (1997)).

At small perturbations TS-1, SO and GS contributions dominate, the interaction of the projectile field with the target can be treated in first order and, consequently, FDCSs should be identical for fast ion and electron impact. Moreover, since the projectile only interacts once with the target atom transferring a momentum $\vec{q}$, this is the symmetry axis for all differential cross sections. Pioneering experimental as well as theoretical differential data have been reported for $100 \mathrm{MeV} / \mathrm{u} \mathrm{C}^{6+}$ impact, where dynamical mechanisms (Bapat et al 1999) as well as signa- 
tures of the correlated initial state (Bapat et al 2000; Keller et al 2000a) have been investigated and evidence for SO was provided.

Recently, first FDCSs have been published for $6 \mathrm{MeV}$ proton on He collisions (Fischer et al 2003). Seemingly well within the perturbative regime $\left(Z_{P} / v_{P}=0.06\right)$ for single ionisation at least, it has been known since long, however, that the ratio of double to single ionisation at this perturbation differs for projectiles with a different sign of the charge (for reviews see e.g. Ullrich et al (1993); McGuire et al (1995); McGuire (1997); Fischer et al (2003) and references therein). Whereas the ratio for positively charged particle impact is largely converged to its asymptotic value for $Z_{P} / v_{P} \leq 0.1$, it is about a factor of two larger for negatively charged projectiles at this perturbation and does not converge until $Z_{P} / v_{P}=0.02$.

Accordingly, as illustrated in Figure 46, the FDCSs for co-planar geometry, symmetric energy sharing of the two electrons $\left(E_{e 1}=E_{e 2}<25 e V\right)$ and $\mathrm{q}=0.5,1.1$ and 1.7, show clear differences for proton (upper row) and electron (lower row) impact, respectively. As general features, in both cases four "islands" were observed which can be identified to be the binary and the recoil peaks, respectively, mirror imaged at the diagonal $\vartheta_{e 1}=\vartheta_{e 2}$ since the electrons are indistinguishable. Dashed lines indicate kinematical situations which are exactly forbidden in the dipole approximation (Section 4.3). Emission along $\vartheta_{e 1}=\vartheta_{e 2}$ is prohibited as well, due to the Fermi nature of the electrons. As mentioned, for a first order projectile-target interaction, the patterns have to be symmetric with respect to $\vec{q}$, i.e. point symmetric in the two-dimensional plots with respect to the dot or bars indicated in the Figure, representing the finite q-ranges that had to be chosen in the experiment in order to increase statistics.

Two main features were observed by Fischer et al (2003): First, proton data are much more symmetric with respect to the momentum transfer direction indicating indeed, that they seem to be converged much earlier to the asymptotic limit even in terms of FDCSs. Higher order contributions, i.e. deviations from the $\vec{q}$-symmetry are clearly identified in the electron data mainly for the "recoil peak" (see Dorn et al 2001, 2003 and Section 4.3). Second, the proton impact data show a much less pronounced "recoil-peak" contribution. It was speculated (Fischer et al 2003), that at intermediate impact parameters the positively charged protons tend to pull the electrons away from their parent atom favouring a clean "binary situation" whereas the electron projectiles tend to push the electron into the atom, causing a stronger recoil-peak (additional interaction with the nucleus) and a more pronounced higher-order behavior. 
Clearly, such an interpretation has to await verification by quantum calculations delivering FDCSs, presently being underway (Dorn et al 2003; Kheifets 2003). Convergence studies of total cross sections (Bronk et al 1998) within the multi-cut "forced impact method" (Ford and Reading 1988; 1990) indicate that positively charged particles do show more "dynamical" or "intermediate" correlation than negatively charged ones, in accordance with the above considerations. Since the electrons are mainly pulled towards the proton projectile (enhanced "binary peak"), they tend to be closer to each other with increased intermediate correlation whereas a negatively charged projectile tends to dilute the two-electron density and, hence, reduces dynamical correlation effects.

At strong perturbations, double ionisation is dominated by the TS-2 and higher-order diagrams in the Born-series. In TS-2, for example, the ion-induced field independently acts on both target electrons giving rise to their ejection. As of now, despite of few attempts for electron impact (see e.g. Dorn et al (2003); Mkhanter and Dal Cappello (1998); Grin et al (2000)), no quantum mechanical FDCSs are at hand that consistently include second-order contributions, not to speak about higher terms in the perturbation expansion. Since the electron emission spectra alone, integrated over all projectile scattering angles, have been found to be unaffected by the interaction between the nuclei (Fainstein et al 1988) they might be described within the independent particle model (IPM) if correlation between electrons is neglected.

Following these lines, Moshammer et al (1997) were able to quantitatively describe single differential cross sections as a function of the energy of one "typical" electron (integrated over all energies of the second) for double ionisation of helium by $1 \mathrm{GeV} / \mathrm{u} \mathrm{U}^{92+}$ impact within the dipole approximation. More recently, Kirchner et al 2002a have developed a much more sophisticated IPM-model, were the impact parameter dependent effective single-particle ionisation probabilities $P_{i}(b)$ for certain sub-shells i are calculated within the CDW-EIS approach. Using some further approximations $\left(P_{i}(b) \equiv 1\right.$ for $P_{i}(b)>1$ or using the unitarity prescription by Sidorovich and Nikolaev (1983)), good agreement has been obtained between experimental and theoretical double differential cross sections (DDCS) for electron emission in neon double ionisation. It was concluded that correlation effects between the electrons, which are completely neglected in the IPM are of minor importance if only a small fraction of all target electrons is emitted i.e. for low final charge states of the target. For the helium target instead, the IPM was found to strongly overestimate the experimental DDCSs at all transverse electron energies, indicating that electron correlation cannot be neglected in this case. 
The full four-body dynamics has been investigated recently by Perumal et al (2002) for double ionisation of helium by $3.6 \mathrm{MeV} / \mathrm{u} \mathrm{Au}^{53+}$ projectiles. Considering the centre-of-mass motion of the two emitted electrons by adding their vector momenta, all dynamical features that have been observed for single ionisation were rediscovered, showing that the four-body problem could be reduced in good approximation to an effective three-particle problem.

As shown in Figure 47a,b, the recoil-ion was found to essentially compensate the sum momentum of both electrons in the longitudinal (a) as well as in the transverse (b) directions (the collision geometry is defined as in Figure 39), with a strong longitudinal backwardforward asymmetry due to PCI. Transversally (b), the projectile deflection was observed to be nearly symmetric around zero, indicating that it is neither dominated by the interaction with the target electrons nor with the nucleus. It has been discussed that these results are surprising on first glance, since the "two-step two" mechanism was commonly thought of as an independent, binary-like interaction of the projectile with both of the electrons where the projectile transverse momentum would have to be largely balanced by the sum momentum of the electrons. Instead, it was concluded from the data and from calculations (Kirchner et al 2002a), that typical impact parameters are such large that the target is "dissociated" in the strong projectile field, with similar but oppositely directed forces acting on both electrons and on the nucleus mainly transferring energy to the target in a dipole-like reaction with quite small $\vec{q}$.

Within such a scenario it is not too surprising that the transverse momenta of both individual electrons display a quite similar distribution as shown in Figure $47 \mathrm{~d}$ in contrast to the longitudinal ones (Figure 47c), where fast and slow electrons are distinguished by the experiment. This indicates first, that longitudinal and transverse momentum components are independent to a large extent and second, as has been suggested by Perumal et al (2002), that a certain realisation of the TS- 2 mechanism might dominate where the momentum transfer to both target electrons and to the nucleus are not independent in each single collision but indeed quite similar in magnitude but into opposite directions due to the large impact parameters involved. Then, both electrons would be effectively "displaced from their nucleus" resulting in a "Coulomb explosion" of the two electrons with their final state correlation then being intimately connected to their correlated initial state.

Indeed, distinct correlations between the electrons have been found in this experiment as well as in previous measurements for $\mathrm{He}$, Ne double as well as Ne triple ionisation (Moshammer et al 1996b). The latter have been discussed and partly explained by classical nCTMC calculations (n-body CTMC, Olson et al (1989)) to result from the final state interaction. Moreover, 
the experiments have triggered calculations within the Weizsäcker-Williams method of equivalent photons (Keller et al 1997) for double ionisation by $1 \mathrm{GeV} / \mathrm{u} \mathrm{U}^{92+}$ impact in the non-perturbative regime (see comments by Voitkiv and Ullrich (2001)). Here, the extremely short, time-dependent electromagnetic pulse created by the projectile is Fourier transformed into the frequency domain and quantized yielding a broad-band virtual photon pulse. Double ionisation in a TS-2 like mechanism then is described by the absorption of two virtual photons, one by each of the electrons and, accordingly, would sensitively depend on the correlated ground-state wave function. Indeed, different wave functions in the calculations resulted in considerably different correlated electron spectra.

Recently, electron correlations have been investigated by inspecting the so-called correlation function R (Schulz et al 2000a; Feuerstein et al 2001a; Gerchikov and Sheinermann 2001; Schulz et al 2001a; Gerchikov et al 2002). Here, the probability to find two electrons emitted in the same multiple ionisation event with a certain momentum difference is compared to the corresponding probability for two independent electrons emitted in two different collisions. It was demonstrated, that the correlation function is neither sensitive on the respective mechanism leading to double ionisation (first-order or TS-2) nor on the final-state postcollision interaction with the projectile, possibly making $\mathrm{R}$ an ideal tool to investigate groundstate properties of the correlated wave function. This was substantiated by Gerchikov and Sheinermann (2001) who analysed the correlation function $R_{C}$ for back-to-back emission of electrons with equal energy. It was found, in qualitative agreement with experiment, that the maximum in $R_{C}$ is very sensitive on the mean initial-state separation between the two electrons and, moreover, its shape strongly depends on the correlated initial-state used in the calculation (see Figure 48).

\subsubsection{Multiple ionisation at large perturbations}

Up to now, only one kinematically complete experiment on multiple ionisation has been performed for $3.6 \mathrm{MeV} / \mathrm{u} \mathrm{Au}^{53+}$ impact on neon (Schulz et al 2000). For a large number of total, single and doubly differential cross section measurements see the previous reviews (Dörner et al 2000; Ullrich et al 1994, 1997). In Figure 49 the momentum vectors of $\mathrm{Ne}^{3+}$ recoil ions are plotted along with the vector sum-momentum of all three emitted electrons. The collision plane is defined as in Figure 39. Not even an attempt has been made up to now to describe the complete five-particle dynamics in a strongly non-perturbative situation within a quantum-mechanical theory. However, classical nCTMC results are at hand for comparison, displayed in the right-hand panel of the Figure. 
Surprisingly, even for triple ionisation the authors found the typical features observed before for single and double electron emission in the non-perturbative regime, namely little netmomentum transfer at considerable energy deposition. As calculations indicate (Kirchner et al 2002a), even for triple ionisation the most likely impact parameters are larger than 3 a.u., well outside the target electron cloud again causing a kind of dissociation of the atom in the field. As before, a strong PCI effect was observed, dragging each of the electrons behind but, at the same time, pushing away the $\mathrm{Ne}^{3+}$ ions with similar momenta. Implying that all the electrons experience about the same PC force the authors tried to separate the influence of the PCI from the (correlated) relative motion of the three electrons by a transformation into the threeelectron centre-of-mass (CM) co-ordinate frame, where PCI should not be present at all under the given assumptions.

In Figure 50 the relative energies of the three electrons $\varepsilon_{e i}=E_{i}^{C M} / \sum E_{i}^{C M}$ in the $\mathrm{CM}$ system ( $E_{i}^{C M}:$ CM energy of the $\mathrm{i}^{\text {th }}$ electron) were presented in a modified Dalitz-plot (Dalitz 1953). This is an equilateral triangle where each triple ionisation event is represented by one point inside the triangle with its distance from each individual side being proportional to the relative energy of the corresponding electron as indicated in the Figure. Only events in the inscribed circle are allowed due to momentum conservation in the CM frame $\left(\sum \vec{P}_{e i}^{C M}=0\right)$. Electrons are numbered such that electron 1 is the one with the smallest angle relative to the projectile propagation direction in each triple ionisation event and electron 3 the one with the largest angle.

Obviously, the electron energies are not independent of each other and the many-electron continuum, explored for the first time experimentally, was found to be strongly correlated. There is an increased probability that electrons 1 and 3 have large energies compared to electron two. nCTMC calculations without inclusion of the electron-electron interaction beyond an effective potential in the initial state were not able to reproduce these structures (Figure 50b). Considering the final state interaction between the electrons in a similar way as by Moshammer et al (1996) led to structures in the Dalitz plot but essentially with the role of electron "two" and "three" exchanged. Introducing in addition a completely correlated, threeelectron (P-electrons neglecting the spin) classical initial state, where the individual electrons move on Kepler ellipses at equal distances relative to each other on the corners of an equilateral triangle in a plane, with the electron-electron interaction "switched on" during the entire collision, brought the theoretical results surprisingly close to the experimental data (Figure 50c). 
Differential energy loss spectra of the projectile were reported for the same collision system for single to six-fold ionisation (Schulz et al 1999) surpassing previously achieved resolutions (Schuch et al 1988; Schöne et al 1995) by an order of magnitude through monitoring all target reaction products up to triple ionisation. It was shown, that an "average electron model", where the energy distribution of a "typical" electron emitted in an $n$-fold ionisation event was $\mathrm{n}$ times convoluted with itself in order to simulate the total energy loss, was in excellent agreement with the directly measured data for up to triple ionisation. Thus, electron spectra measured in coincidence with n-times charged recoil ions were used to extract absolute energy loss distributions for up to six-fold ionisation and reasonable agreement with nCTMC calculations was observed. Finally, a technique was suggested, to determine the contribution of target excitation to the total energy loss.

\subsubsection{A Short Summary}

Essentially within the last five years, momentum spectroscopy of ions and electrons has boosted traditional ion-atom collision physics in a remarkable way. The Q-value resolution in electron capture reactions at low collision velocities has reached a precision in the energy level determination competing with the most accurate spectroscopic techniques and further improvements are expected. Electron capture at high velocities has been explored, partly in a storage ring, giving unique information on second order "Thomas" contributions. Electron impact ionisation of ions has been observed for the first time in fully differential measurements by exploiting inverse kinematics and an event-by-event identification of the (e-e) contribution to projectile ionisation in fast ion-atom collisions. Unprecedented impact-parameter dependent ultra-low energy electron spectra have been recorded in slow ion-atom collisions visualizing quasi-molecular orbitals formed during the collision and helping to clarify a long standing debate on "saddle-point electrons". Relativistic collisions with light targets were started to be explored and relativistic effects have been observed. 3D imaging of emitted electrons in kinematically complete experiments on single ionisation in the perturbative regime revealed surprises for the fundamental three-particle Coulomb system that have never been observed before in three decades of $(\mathrm{e}, 2 \mathrm{e})$ research for electron impact, challenging theory considerably. Non-perturbative situations at large velocities became accessible for swift highly-charged ion impact delivering benchmark data for the comparison with the most sophisticated non-perturbative theories. The long-discussed difference in the ratio of double to single ionisation cross sections by projectiles of different sign of the charge has been started to be studied in terms of fully differential cross sections. Triple ionisation was explored in kinematically complete measurements observing correlations in the three-electron continuum, 
the correlation function has been investigated for double as well as triple ionisation and energy loss measurements for multiply ionising ion-atom collisions were reported for up to sixfold ionisation on the basis of single collision experiments.

\section{A View into the Future}

Rapid progress in the next future can be expected in all the fields described in this review and it was partly envisaged at the end of each section. Expecially in laser physics, however, where only three groups world-wide have made use of Reaction Microscopes until now, a whole bunch of investigations might become feasiable: Among them are differential (see e.g. Hasegawa et al (2001); Yamanouchi (2002)) as well as kinematically complete measurements on molecules (a first one has been reported recently by Rottke et al (2002), see also Staudte et al (2002), Alnaser et al (2003)), on state-prepared molecular ions or experiments allowing to extract fully differential cross sections as routinely done for photon, electron or ion impact. Furthermore, investigations using extremely short laser pulses, with two or three optical cycles only (see e.g. Morgner et al 2001) and fixed phase within the envelope (Baltuska et al 2003) or with attosecond higher-harmonic pulses (Drescher et al 2001), will certainly be performed. Moreover, pulses will be actively shaped or sequences of pulses will be applied in the future (see e.g. Wollenhaupt et al (2002)) in order to coherently control the electron dynamics such that certain reactions in atoms, molecules or clusters will be either enhanced or suppressed.

A major step forward will be the advent of tuneable high-intensity short-pulse VUV or even x-ray self-amplifying (SASE) free-electron lasers (FEL). Such a machine has been demonstrated recently and first experiments on the fragmentation of clusters have been performed (Wabnitz et al 2002). At the TESLA-Test Facility in Hamburg $150 f_{s}$, $10^{17} \mathrm{~W} / \mathrm{cm}^{2}$ pulses will be available in 2004 at photon energies between $20 \mathrm{eV}$ and $200 \mathrm{eV}$, at a bandwidth of $10^{-4}, 70 \mathrm{kHz}$ repetition rate and, if demanded, synchronized with a conventional high-intensity $f_{S}$ Ti:Sa laser. Unique experiments will become feasible with this machine on the non-linear interaction of coherent high-energy light with ions, atoms, molecules, molecular ions, clusters and surfaces. Some of the schemes (U1lrich et al 2003a), making use of the optical laser phase synchronized with the FEL pulse, envisage to monitor the time-evolution of correlated atomic and molecular electronic processes on an attosecond time-scale applying methods that have been used to characterize attosecond pulses recently (Drescher et al 2002). 
Moreover, for ion impact first kinematically complete experiments on multiple ionisation at high energies, up to $500 \mathrm{MeV} / \mathrm{u}$, and for projectile charge states between about 30+ and 92+, i.e. at large perturbations $Z_{P} / v_{p}$, will soon be feasible in the experimental storage ring ESR of GSI in Darmstadt. Up to now, only one Recoil-Ion Momentum Spectrometer has been implemented into a storage ring, the Stockholm CRYRING. At GSI a Reaction Microscope has been developed and will become operational in 2003. Due to the strongly increased luminosity in the ring as compared to single pass experiments we expect considerable, ordersof-magnitude, increased event-rates, such that fully differential cross sections should become measurable for double, triple and even quadruple ionisation in attosecond fields.

With the adaptation of the concepts of Reaction Microscopes to the imaging of electrons (Hattas et al 2003, Moshammer and Ullrich 1998) and ions (Moshammer et al 1990, Jalowy et al 2002; 2002a,b; 2003; 2003a) emitted from surfaces this successful technique has just entered the field of solid state and surface physics. Here, a similar stormy progress as the one in atomic and molecular physics can be expected for the future. One promising route is to investigate the correlated emission of electrons from solids by single photon, laser or ion impact, analogous to the experiments described in this review for atomic targets.

The tools described here are ideally suited for the investigation of the orientation dependence and the reaction-pathways of slow molecule-atom collisions. First exploratory experiments (Wu et al 1998) already showed that this technology has the potential to provide substantial contributions to reaction stereochemistry.

Laser assisted collisions, that have been theoretically explored since a while (see Ehlotsky et al (1998) for a recent review and refences in Section 4.3) will certainly be an upcoming field after the first feasibility demonstration in electron-atom collisions (see Scetion 4.3) using a Reaction Microscope along with an intense, ns-pulsed YAG laser. Until now, such studies were only accessible experimentally for elastic and resonant scattering. Unexpectedly strong coupling of an even weak $\left(E_{0}=0.005\right.$ a.u. $)$, low-frequency $\left(\omega_{0}=0.004 a . u\right.$. $)$ electromagnetic radiation field to matter has been recently predicted in laser-assisted collisions considering a direct encounter of a fast $\left(v_{P}=12 a . u\right.$. $)$ proton with a target electron, so-called binary encounter electrons (BEE; Voitkiv and Ullrich 2001a, 2001b). Whereas the laser field used in the calculation was by far not strong enough to noticeably disturb the hydrogen target-atom ground-state alone, strong effects occur during the collision in the high-energy BEE emission: Thousands of laser-photons were observed to couple to the system, strongly modifying the energy and angular distribution of the BEE. Moreover, electron transfer reactions in slow ion- 
atom collisions were calculated to be considerably affected by a laser-field, possibly opening the door for ultra-fast control of electronic motion within one optical half-cycle (Kirchner 2002).

Another interesting situation arises, and might be realised with the PHELIX laser at GSI (PHELIX 2001), if both, attosecond ion-induced fields and femtosecond strong laser fields act together. The ion-induced pulse efficiently brings a large number of electrons into the continuum, placing them "simultaneously" with little energy into the oscillating field of the laser, which then accelerates this bunch of electrons very effectively in a coherent way heating them tremendously. Thus, one might envisage, that the most effective way to transfer energy to matter might be a concerted action between ion-induced and laser fields.

Acknowledgements: We gratefully acknowledge support from the Max-Planck-Society, from the Deutsche Forschungsgemeinschaft DFG within the Leibniz-Programm and several separate proposals, from the Bundesministerium für Forschung und Technologie BMFT, from the Deutscher Akademischer Austauschdienst DAAD and from GSI. Last not least, we would like to thank numerous students, who have contributed over the the years, many colleagues for stimulating discussions and exciting new experimental ideas. 


\section{Figure Captions}

Figure 1: Illustration of the momenta occurring in atomic fragmentation by projectile impact (see text). From Ullrich and Shevelko (2003).

Figure 2: Different reaction channels that can occur in an ion-atom collision (see text). From Ullrich and Shevelko (2003).

Figure 3: The $Q$-value or longitudinal recoil-ion momentum spectrum for $\mathrm{Ne}^{6+}$ measured for single capture in $\mathrm{Ne}^{7+}$ on $\mathrm{He}$ collisions at $v_{p}=0.335 a$.u. The resolution is 0.52 $\mathrm{eV}$. The scale of the energy levels, counted from the ground state of $\mathrm{Ne}^{6+}$ is also shown together with the identification of the $2 s 4 l^{1,3} \mathrm{~L}$ singlet and triplet states $(\mathrm{L}=$ S, P, D and F). Upper marks: Multi-configuration Hartree-Fock calculations (Buchet-Poulizak et al 2001). Lower marks with error bars: Experimental result by Fischer et al (2002).

Figure 4: Momentum distributions for single ionisation in collisions with photons propagating along the z-axis for linear polarization along the x-direction. (a): Single ionisation of $\mathrm{He}$ by absorption of single $80 \mathrm{eV}$ photons. In the Figure, the recoil ion momentum distributions are integrated over $P_{R z}^{f}=0 \pm 0.1$ a.u. and $p_{\text {rec }}=P_{R}^{f}$. From Dörner et al (1997). (b): Electron momentum distribution for multi-photon single ionisation of Ar by $25 \mathrm{fs}$ laser pulses at $780 \mathrm{~nm}$ and an intensity of a few $10^{13} \mathrm{~W} / \mathrm{cm}^{2}$. The momentum distributions represent a cut along the z-direction of $P_{R z}^{f}=0 \pm 0.1 a . u$. (c): Recoil-ion momentum distribution for tunnelling single ionisation of $\mathrm{Ne}$ interacting with $1 \cdot 10^{15} \mathrm{~W} / \mathrm{cm}^{2}, 25 \mathrm{fs}$ laser pulses at $780 \mathrm{~nm}$. All events along $\mathrm{z}$ are projected. From Moshammer et al (2000). (d): Recoil-ion momentum distribution created by $7 \mathrm{keV}$ linear polarized photon impact, outer rim: photo absorption, narrow peak: Compton scattering $\left(k_{z r e c}=P_{R x}^{f}, k_{y r e c}=P_{R y}^{f}\right)$ From Spielberger et al 1995.

Figure 5: Reaction Microscope. From Moshammer et al (2003b).

Figure 6: Time-of-flight spectrum for ionisation of Ar with $25 f_{s}$ laser pulses at an intensity of some $10^{13} \mathrm{~W} / \mathrm{cm}^{2}$. The inset shows a blow up of the $\mathrm{Ar}^{1+}$ TOF-peak (see text).

Figure 7: Projection of an electron trajectory onto a plane perpendicular to the magnetic field (view along the magnetic field lines). From Moshammer et al (2003b). 
Figure 8: The electron radial displacement $\mathrm{r}$ on the detector versus the electron time-of-flight for for single ionisation of $\mathrm{H}_{2}$ by $6 \mathrm{MeV}$ proton impact. The cyclotron revolution time corresponds to a magnetic field strength of 14 Gauss. The solid lines are the calculated displacements $r$ for electrons with kinetic energies between 10 and $50 \mathrm{eV}$ in steps of $10 \mathrm{eV}$. The point $(T O F=120 \mathrm{~ns}, r=0)$ represents the origin $\left(E_{e}=0\right)$ on the electron energy scale. From Moshammer et al (2003b).

Figure 9: Density plots of projections of the momentum distributions from double ionisation of He for three different energies. From left to right: Data sets for $1 \mathrm{eV}, 20 \mathrm{eV}$ and $100 \mathrm{eV}$ above threshold. The $\mathrm{z}$ and y components of the momentum are plotted on the horizontal and vertical axes, respectively. The polarization vector of the photon is in the $\mathrm{z}$ direction and the photon propagates in the $\mathrm{x}$ direction perpendicular to $\mathrm{y}$ and z. For $100 \mathrm{eV}$ above threshold only events with $-1 a . u .<k_{x}<1 a . u$. are projected onto the plane. (a),(d),(g): Momentum distributions of the $\mathrm{He}^{2+}$ ion $\left(k_{+}\right)$ for $1 \mathrm{eV}, 20 \mathrm{eV}$ and $100 \mathrm{eV}$ above threshold. (b),(e),(h): Electron momentum $\left(k_{1}\right)$ and (c),(f),(i) electron pair relative momentum $\left(k_{1}-k_{2}\right) / 2$. The circle locates the maximum possible momentum in each coordinate at the respective photon energy (from Dörner et al (1996); Bräuning et al (1997); Knapp et al (2002a)).

Figure 10: Photo double ionisation of $\mathrm{He}$ at $20 \mathrm{eV}$ above threshold by linear, left and right circular polarized light. Shown is the momentum distribution of electron "two" for fixed direction of electron "one" as indicated by the arrow. The plane of the Figure is the momentum plane of the three particles. The data of (a) are integrated over all orientations of the polarization axis with respect to this plane. The Figure samples the full cross sections, for all angular and energy distributions of the fragments. The outer circle corresponds to the maximum possible electron momentum, the inner one to the case of equal energy sharing. In (b) and (c) the light propagates into the plane of the Figure, the electrons are confined to the plane perpendicular to the light propagation (from Dörner et al (1998b) and Achler et al (2001)).

Figure 11: Fully differential cross section of the He photo double ionisation at $529 \mathrm{eV}$ photon energy. The primary photoelectron "one" emission direction is indicated by the arrow, the polarization is horizontal, the angular distribution of the complementary electron "two" with energy $E_{2}$ is given by the symbols. a) $447 \mathrm{eV}<E_{1}<450 \mathrm{eV}$, $0 \mathrm{eV}<E_{2}<3 \mathrm{eV}$. b) $410 \mathrm{eV}<E_{1}<430 \mathrm{eV}, 20 \mathrm{eV}<E_{2}<40 \mathrm{eV}$. a) shows the 
dominance of shake-off, the $90^{\circ}$ emission in b) indicates the importance of TS1 at this energy. The solid line shows the full CCC calculation, the dashed line is the shake-off only part of the CCC calculation (from Knapp et al 2002).

Figure 12: Left: Simplified potential energy diagram for $\mathrm{NO}^{+}$. Right: Electron and $\mathrm{N}^{+}$-ion kinetic energy correlation for photo ionisation of $\mathrm{NO}$ at $23.64 \mathrm{eV}$ photon energy. The three numbered island correspond to the three reaction pathways shown on the left panel (from Lafosse et al 2000).

Figure 13: Angular distribution of K-shell photoelectrons at $10 \mathrm{eV}$ electron energy. (A)-(E): carbon-K shell electrons from $C O$ molecule, linearly polarized light, the orientation of the molecule and the polarization vector is indicated in the Figure. The black dot is the carbon, the grey dot is the oxygen end of $C O$ (from Landers et al (2001)). The full line is a fit of spherical harmonics for $(F, G)$ left and right circular polarized light. $C O$ orientation along the rod as indicated. The direction of the photon is shown by the spiral. $(\mathrm{H})$ as $(\mathrm{F}, \mathrm{G})$ but for $\mathrm{N}_{2}$ (from Jahnke et al 2002).

Figure 14: Angular distribution of carbon-K-Auger electrons from $\mathrm{CO}^{+}$. (a) For a $\Pi$ and $\Delta$ transition $\left(C O^{+}\left({ }^{1} \Sigma^{+}\right) \rightarrow C O^{2+}\left({ }^{1} \Delta,{ }^{3} \Pi,{ }^{1} \Pi\right.\right.$ transition $)$. b) corresponds to a $\mathrm{CO}^{+}\left({ }^{1} \Sigma^{+}\right) \rightarrow \mathrm{CO}^{2+}\left({ }^{1} \Sigma\right)$ transition. c) same data as in b): $0^{\circ}$ corresponds to emission in direction of the carbon. The full lines in all Figures are fits of Legendre polynomials to guide the eye. Other lines in c) are results of a multiple scattering calculation for an $\mathrm{S}$ electron wave starting at the carbon center in $\mathrm{CO}^{2+}$. Dashed: both vacancies in the $\mathrm{CO}^{2+}$ at $\mathrm{C}(2 \mathrm{p})$. Dotted: One vacancy each at $\mathrm{C}(2 \mathrm{p})$ and $\mathrm{O}(2 \mathrm{p})$. The absolute height of the calculation is arbitrary (from Weber et al 2003).

Figure 15: Longitudinal and transverse momentum distribution of $\mathrm{Ne}^{1+}$ recoil ions (1a) and of electrons (1b) for single ionisation by $1 \cdot 10^{15} \mathrm{~W} / \mathrm{cm}^{2}, 25 \mathrm{f}_{S}$ laser pulses at $800 \mathrm{~nm}$. The z-scale is linear with the box sizes proportional to the number of events at a given momentum. From Moshammer et al (2003a).

Figure 16: Longitudinal momentum distribution of the emitted electron $\left(P_{e \|}\right)$ for the same conditions as in Figure 15. Thin line: Prediction of tunnelling theory (Delone and Krainov 1991) for $0.7 \cdot 10^{15} \mathrm{~W} / \mathrm{cm}^{2}$. Thick line: Prediction of semi-classical calculations at $0.7 \cdot 10^{15} \mathrm{~W} / \mathrm{cm}^{2}$ for He single ionisation (Chen and Nam 2002). From Moshammer et al (2003a). 
Figure 17: Ne double ionisation by $780 \mathrm{~nm}, 25 f_{S}$ fs laser pulses. Left: $\mathrm{Ne}^{1+}, \mathrm{Ne}^{2+}$ yields as a function of the laser intensity (from Larochelle et al (1998)). Solid lines: Independent event model. Right hand side: ion momentum distributions for different intensities (see text; from Dörner et al (2002)).

Figure 18: Correlated electron momenta along the polarization axis for double ionisation of Ar at a) $3.8 \cdot 10^{14} \mathrm{~W} / \mathrm{cm}^{2}$ (nonsequential regime) and b) $1.5 \cdot 10^{15} \mathrm{~W} / \mathrm{cm}^{2}$ (sequential regime). From Weber et al 2000b.

Figure 19: Correlated electron momenta along the polarization axis for double ionisation of $\mathrm{Ne}$ (a) at $1 \cdot 10^{15} \mathrm{~W} / \mathrm{cm}^{2}$. Full lines: Kinematical boundaries for classical rescattering. From Moshammer et al (2003). b): Same as a) for $\mathrm{Ar}^{2+}$ creation at $0.25 \cdot 10^{15} \mathrm{~W} / \mathrm{cm}^{2}$ (Feuerstein et al (2001)). Full line: Classical boundaries for recollision. Dotted line: Kinematical limit for excitation during recollision followed by field ionisation in one of the subsequent laser cycles.

Figure 20: Correlated momenta of electrons for $\mathrm{He}^{2+}$ (left, theory by Lein et al (2000)) and $\mathrm{Ar}^{2+}$ (right, experiment (Moshammer et al 2002) creation at indicated laser intensities. Experiment: the transverse momentum of electron 1 is larger than 0.5 a.u.

Figure 21: Ion momentum distributions along the laser polarization direction for He (Weber et al 2000), Ne (Moshammer et al 2000) and Ar (Eremina et al 2003). Respective intensities are indicated in each panel. Upper right number in the panels: Ratio of recollision energy to the ionisation potential of the singly charged ion without field.

Figure 22: Electron momentum distribution in the plane perpendicular to the polarization for double ionisation of Ar at $1.9 \cdot 10^{14} \mathrm{~W} / \mathrm{cm}^{2}$. The direction of the first electron is indicated by the arrow, the perpendicular momentum of the second electron is shown. The data are integrated over the momentum components in field direction. (a) experiment, (b) theoretical results including e-e momentum exchange in the recollision and e-e repulsion after the recollision. (c) theoretical results not including final state repulsion. From Weckenbrock et al 2003.

Figure 23: Triple differential cross section (TDCS) in coplanar scattering geometry for single ionisation of helium by $500 \mathrm{eV}$ electron impact as a function of the emission angle 
$\Theta_{b}$ and the momentum transfer $|\vec{q}|$ of the ionised electron $\left(E_{b}=5 \mathrm{eV}\right)$. White curves: direction of the momentum transfer $|\vec{q}|$ (left) and $-|\vec{q}|$ (right).

Figure 24: TDCS for $|\vec{q}|=0.6$ a.u. (direction of $\vec{q}$ indicated by an arrow) as a function of the longitudinal and transversal momentum of the ejected electron.

Figure 25: FDCS in coplanar scattering geometry as function of the ejected electron emission angles $\Theta_{b}$ and $\Theta_{c}$ relative to the primary beam forward direction. The ejected electron energies are $E_{b, c}=5 \pm 2 \mathrm{eV}$. a) Experimental cross section for $E_{0}=2 \mathrm{keV}$, $|\vec{q}|=0.5 \pm 0.2 a . u .$. b) The electron emission configurations for the cross section maxima (A) and (B) in a). c) Photo double ionisation cross section for $E_{b, c}=5 \mathrm{eV}$. d) The electron emission configurations for the cross section maxima (A) and (B) in c). e) CCC calculation for $|\vec{q}|=0.5$ a.u. . The direction of the momentum transfer $\vec{q}$ is marked by arrows. In both diagrams the angular range which is not affected by the experimental detector dead time is encircled by solid lines (see text). From Ullrich and Shevelko (2003).

Figure 26: FDCS for $E_{0}=2 \mathrm{keV},|\vec{q}|=2 a . u$. and fixed emission angle $\Theta_{c}=0^{\circ} \quad\left(E_{b}=5 \mathrm{eV}\right.$, $E_{c}=25 \mathrm{eV}$ ). Solid line: Convergent close coupling (CCC) calculation. Dashed line: Calculation using the $3 \mathrm{C}$ final state wave function. From Dorn et al (2002a).

Figure 27: FDCS for DI by $E_{0}=500 \mathrm{eV}$ electron impact and $|\vec{q}|=0.7 \pm 0.2 \mathrm{a} . u$. but otherwise unchanged conditions as for Figure 24a. The full square on the diagonal line indicates the momentum transfer direction. From Dorn et al (2003).

Figure 28: Transversal momentum distributions of the ionised electrons (a) and the recoiling ions (b) for single ionisation $(\mathrm{e}, 2 \mathrm{e})$, excitation-ionisation $(e, \gamma 2 e)$ and double ionisation $(\mathrm{e}, 3 \mathrm{e})$.

Figure 29: Cross section for electron impact ionisation of helium differential in the longitudinal momentum of the residual $\mathrm{He}^{+}$ions parallel to the incoming projectile beam for the laser beam on and off.

Figure 30: Transverse momentum transferred to the recoil ion versus $Q$-value for single electron capture in $\mathrm{Ar}^{16+}-\mathrm{He}$ collisions at different projectile velocities. The principal quantum numbers $n$ of populated states are marked as vertical lines. The transverse 
recoil momentum correspond to projectile scattering angles $\theta_{P}=P_{R \perp} / P_{0}$, with $P_{0}$ the initial projectile momentum. The dashed lines indicate $\theta_{P}=\theta_{C}$ (half Coloumb angle). From Abdallah et al (1998a).

Figure 31: $Q$-value spectrum and level energies relative to the ground state of $\mathrm{Ne}^{6+}$ following single electron capture in $\mathrm{Ne}^{7+}-\mathrm{He}$ collisions at $63 \mathrm{keV}$. From Fischer et al (2002).

Figure 32: Angular scattering pattern $\sigma(\theta)$ of Li-atoms emerging from electron transfer collisions at $6 \mathrm{keV}$ with cold $\mathrm{Na}$, deduced from the measured transverse recoil-ion momentum distribution. Fraunhofer-type rings in the angular distribution are clearly resolved (from Van der Poel et al 2001).

Figure 33: Recoil-ion longitudinal momentum distributions converted into $Q$-values for double electron capture from $\mathrm{He}$ in collision with $\mathrm{Ne}^{10+}$ at $50 \mathrm{keV}$ (upper part) and $150 \mathrm{keV}$ (lower part). The result for TI is plotted on the left and true double capture on the right side. The labels denote the quantum numbers ( $\left.n l, n^{\prime} l l^{\prime}\right)$ of populated doubly excited states. From Flechard et al (2001a).

Figure 34: Longitudinal momentum distributions of recoil ions at fixed scattering angles for simultaneous transfer and ionisation in proton He collisions (doubly differential cross section $\left.d \sigma /\left(d \theta_{P} d P_{R \|}\right)\right)$. The scattering angle $\theta_{P}$ is 0.15 (left column) and $0.55 \mathrm{mrad}$ (right column). Left vertical lines: expected $P_{R \|}$ for kinematical capture. Solid curves: results of an independent electron approximation. From Mergel et al (1997).

Figure 35: Schematic illustration of the kinematics for $(e, e)$ and $(n, e)$ contributions to projectile ionisation. From Kollmus et al (2002).

Figure 36: Azimuthal angle between projectile electron and target $\mathrm{He}^{1+}$ recoil-ion $\Phi(n, e)$ versus azimuthal angle between emitted target and projectile electrons $\Phi(e, e)$ for 3.6 MeV/u $\mathrm{C}^{2+}$ on $\mathrm{He}\left(1 \mathrm{~s}^{2}\right)$ collisions (left column: experiment; middle column: CTMC) and for $3.6 \mathrm{MeV} / \mathrm{u} \mathrm{C}^{2+}$ on $\mathrm{H}(2 \mathrm{~s})$ collisions (right column: CTMC). Z-scale is logarithmic with ten steps from the minimum to the maximum cross section in each column represented by different sizes of the symbols. Upper row: All events. Middle row: $\left|\vec{P}_{e}\right| \geq\left|\vec{P}_{R}\right|$. Lower row: $\left|\vec{P}_{e}\right| \leq\left|\vec{P}_{R}\right|$. From Kollmus et al (2002). 
Figure 37: Scaled momentum transfer $q^{*}$ (see text) versus angle between $\vec{q}$ and the emitted electron $\vartheta_{e}$ in co-planar geometry for $\mathrm{C}^{2+}$ ionisation (upper part) in inverse kinematics for simultaneous projectile and target ionisation with $\left|\vec{P}_{e}\right| \geq\left|\vec{P}_{R}\right|$ and He ionisation (lower part) by $2 \mathrm{keV}$ electron impact (see text). Z-scale is logarithmic with ten steps from the minimum to the maximum cross section in each column represented by different sizes of the symbols. From Kollmus et al (2002).

Figure 38: Electron velocity distributions in different planes normalized to the projectile velocity $v_{P}$. The Cartesian coordinate system is defined by the beam axis $(\mathrm{z})$, the transversal momentum transfer to the recoil ion (-y) and the direction perpendicular to the nuclear scattering plane $(\mathrm{x})$. a) Projection onto the scattering plane $(y, z)$, "topview" for the reaction $\mathrm{p}+\mathrm{He} \rightarrow \mathrm{p}+\mathrm{He}^{+}+\mathrm{e}$ at $v_{P}=0.63$ a.u. (Dörner et al 1996a). b) Topview projection for the reaction $\mathrm{Ne}^{+}+\mathrm{Ne} \rightarrow \mathrm{Ne}^{+}+\mathrm{Ne}^{+}+\mathrm{e}$ at $v_{P}=0.35$ a.u. (Abdallah et al 1998b). c) Projection of a slice with $0.3<v_{z} / v_{P}<0.7$ onto the plane perpendicular to the beam direction (recoil ion moving downward), "frontview" for the reaction $\mathrm{He}^{2+}+\mathrm{He} \rightarrow \mathrm{He}^{+}+\mathrm{He}^{2+}+\mathrm{e}$ at $v_{P}=0.84$ a.u. (Schmidt 2000). d) Topview projection for the reaction $\mathrm{He}^{2+}+\mathrm{H}^{2} \rightarrow \mathrm{He}^{+}+\mathrm{p}+\mathrm{p}$ +e (Afaneh et al 2002).

Figure 39: Dynamics for $6 \mathrm{MeV}$ proton on helium collisions. (a): Schematic illustration. $\vec{P}_{p}^{i}, \quad \vec{P}_{p}^{f}$ : Incoming and scattered ion momentum, respectively, with momentum transfer $\vec{q}=\vec{P}_{P}^{i}-\vec{P}_{P}^{f}$ and $q_{\min }=q_{\|}=\Delta E_{P} / v_{P}$ the minimum momentum transfer ( $\Delta E_{P}$ : projectile energy loss). (b) Experimental results: Two-dimensional finalstate momentum distributions for the recoiling $\mathrm{He}^{+}$target ion, the electron and momentum change of the projectile in singly ionising collisions (logarithmic yscale). The projectiles propagate along the z-direction; their field mainly acts along the $\mathrm{x}$-axis.

Figure 40: Fully differential cross section (FDCS) in arbitrary units for target electrons with defined energy $\left(E_{e}=6.5 \mathrm{eV}\right)$ at a fixed momentum transfer $\vec{q}$ with $|\vec{q}|=0.75$ a.u. as a function of the azimuthal $\left(\varphi_{e}\right)$ and polar $\left(\vartheta_{e}\right)$ electron emission angles, respectively (Schulz et al 2003). The initial projectile momentum $\vec{P}_{P}^{i}$ directed along the 
z-axis in Figure 39 now points upwards for better 3D illustration of the emission pattern. (a): Experiment. (b): Theory (see text).

Figure 41: Longitudinal momentum distributions for electrons emitted in singly ionising 1 $\mathrm{GeV} / \mathrm{u} \mathrm{U}^{92+}$ on He collision. Circles and squares: Experiment; solid and dotted curves: Relativistic first Born and dipole approximation, respectively; dashed curve: Non-relativistic FBA $(c \rightarrow \infty)$. Upper part: Emitted electrons with transverse momenta restricted to $p_{e \perp}<3.5$ a.u.; lower part: $p_{e \perp}<0.25 a . u$. (Voitkiv et al 2002).

Figure 42: Same as Figure 39 (left column) as well as singly differential cross sections for $\mathrm{He}^{+}$ions and electrons as a function of the longitudinal momentum (right column) for different projectile charges and impact energies (perturbation strengths as indicated in the Figure) in singly ionising collisions (logarithmic z-scale). The projectile propagates along the $P_{\|}$direction. Full lines: CDW-EIS results from O’Rourke et al (1997).

Figure 43: Doubly differential cross sections $D D C S=d^{2} /\left(d p_{\|} d p_{\perp} 2 \pi d p_{\perp}\right)$ for electron emission in singly ionising $3.6 \mathrm{MeV} / \mathrm{u}\left(v_{P}=12 a . u\right.$. $) \mathrm{Au}^{53+}$ on $\mathrm{Ar}$ collisions as a function of the longitudinal momentum $d p_{\|}$for fixed transverse momentum transfers $p_{\perp}$ as indicated in the Figure. Open circles: experiment. Full line: CDWEIS (see text). DDCS at different $p_{\perp}$ are multiplied by factors of 10 , respectively. Inset: Theoretical results for different subshells and sum of all contributions (Moshammer et al 1999).

Figure 44: Doubly differential cross sections $D D C S=d^{2} /\left(d q_{\perp} d E_{e}\right)$ as a function of the projectile transverse momentum transfer for specified electron energies for single ionisation of $\mathrm{He}$ by (a) $100 \mathrm{MeV} / \mathrm{u} \mathrm{C}^{6+}$ impact $\left(Z_{P} / v_{P}=0.1\right)$ and (b) $3.6 \mathrm{MeV} / \mathrm{u}$ $\mathrm{Au}^{53+}$ impact $\left(Z_{P} / v_{P}=4.4\right)$. (a) Solid line: FBA; dotted line: Convolution of DDCS for $E_{e}=10 \mathrm{eV}$ with the experimental resolution $\Delta q_{\perp}=0.2$ a.u. (b) Solid line: CDW-EIS with screened nucleus-nucleus interaction; dashed lines: Standard CDW-EIS (Moshammer et al 2001).

Figure 45: FDCS as a function of the polar angle for electrons of $E_{e}=17.5 \mathrm{eV}$ (top) and $E_{e}=55 \mathrm{eV}$ (bottom) emitted in $3.6 \mathrm{MeV} / \mathrm{u} \mathrm{Au}^{53+}$ on He singly ionising collisions in 
coplanar geometry for momentum transfers (left to right rows) of $0.65,1.0$, and 1.5 a.u. . Dashed curve: FBA; solid curve: CDW-EIS (Schulz et al 2002).

Figure 46: Angular distribution of the two emitted electrons $\left(\vartheta_{1}, \vartheta_{2}:\right.$ polar angle with respect to the forward beam direction) in co-planar geometry for proton (a-c) and electron (d-f) impact at a momentum transfer of 0.2 to 0.8 a.u. (a,d), 0.8 to 1.4 a.u. (b,e) and 1.4 to 2.0 a.u. (c,f), respectively for $E_{e 1}=E_{e 2}<25 e V$ symmetric energy sharing. Selected momentum transfer windows are indicated by the black dot or bars, respectively. Symmetry axis in the FBA for the centre of gravity of the momentum transfer: black line. Nodes for dipole transitions: dashed lines. Dotted lines encircle regimes with full momentum acceptance (Fischer et al 2003).

Figure 47: Longitudinal (a) and transverse (b) momentum distributions for the recoil-ions, the sum of both electrons along with the momentum change of the projectile. In Addition, longitudinal (c) as well as transverse (d) momentum distributions for electron "one" and "two" for double ionisation of He by $3.6 \mathrm{MeV} / \mathrm{u} \mathrm{Au}{ }^{53+}$ (Perumal et al 2002).

Figure 48: Correlation function $\mathrm{R}_{\mathrm{C}}$ for double ionisation of $\mathrm{He}$ (open circles) and $\mathrm{Ne}$ (full circles) in collisions with $3.6 \mathrm{MeV} / \mathrm{u} \mathrm{Au}^{53+}\left(v_{P}=12\right.$ a.u. $)$ as a function of the electron momentum difference $\Delta P=\left|\vec{P}_{e 1}-\vec{P}_{e 2}\right|$ for back-to-back emitted electrons. Solid line: Correlated 16-term wave function for the He ground state. Dashed line: 3-term multi-configuration Hartree-Fock wave function (Gerchikov et al 2002).

Figure 49: Two-dimensional final state momentum distributions for the $\mathrm{Ne}^{3+}$ recoil ions and the sum-momentum vector of all three emitted electrons for triple ionisation of $\mathrm{Ne}$ by $3.6 \mathrm{MeV} / \mathrm{u} \mathrm{Au}^{53+}$ impact. The collision plane is defined as in Figure 39. Left side: Experiment. Right side: nCTMC . Z-scale is logarithmic (Schulz et al 2000).

Figure 50: Dalitz-representation (see text) of the energy partitioning of three electrons emitted in triply ionising 3.6 MeV/u Au ${ }^{53+}$ on Ne collisions in the electron centre-of-mass (CM) co-ordinate system. $\varepsilon_{i}$ : energy of the $\mathrm{i}^{\text {th }}$ electron in the CM system. Electrons are numbered according to their emission angle with respect to the projectile direction (see text). Left: Experiment; middle: nCTMC without electronelectron interaction; right: nCTMC with fully correlated three-electron initial-state (Schulz et al 2000). 


\section{References}

Abdallah M, Kravis C D, Cocke C L, Wang Y, Rodriguez V D, Stöckli M 1997 Phys. Rev. A 562000

Abdallah M, Cocke C L, Wolff W, Wolf H, Kravis C D, Stöckli M, Kamber E 1998 Phys. Rev. Lett. 813627

Abdallah M, Wolff W, Wolff H E, Kamber E Y, Stöckli M and Cocke C L 1998a Phys. Rev. A 582911

Abdallah M, Wolff W, Wolff H E, Cocke C L, Stöckli M 1998b Phys. Rev. A. 583379

Abdallah M, Wolff W, Wolff H E, Cocke C L, Stöckli M 1999 J. Phys. B 324237

Achler M, Mergel V, Spielberger L, Azuma Y, Dörner R and Schmidt-Böcking H $2001 J$. Phys. B 34 L965

Adoui L, Caraby C, Cassimi A, Lelièvre D, Grandin J P and Dubois A 1999 J. Phys. B 32631

Adoui L, Tarisien M, Rangama J, Sobocinski P, Cassimi A, Chesnel J Y, Frémont F, Gervais B, Dubois A, Krishnamurthy M, Kumar S and Mathur D 2001 Physica Scripta T92 89

Afaneh F, Dörner R, Schmidt L, Weber Th, Stiebing K E, Jagutzki O, Schmidt-Böcking H 2002 J. Phys. B 35 L229

Ali R, Frohne V, Cocke C L, Stöckli M, Cheng S and Raphaelian L A 1992 Phys. Rev. Lett. 692491

Ali I, DuBois R D, Cocke C L, Hagmann S, Feeler C R and Olson R E 2001 Phys. Rev. A 64 022712

Alnaser A S, Osipov P, Benis E P, Wech A, Cocke C L, Tong X M, and Lin C D 2003 Phys. Rev. Lett. (submitted)

Avaldi L, Camilloni R, Multari R, Stefani G, Robaux O, Tweed R J and Vien G N 1998 J. Phys. B 312981

Balashov V V and Bodrenko I V 1999 J. Phys. B 32 L687, see corrigendum in J. Phys. B 2000331473

Baltuska A et al 2003 Nature 421611

Bandarage G, Parson R 1990 Phys. Rev. A 415878

Bapat B, Moshammer R, Keller S, Schmitt W, Cassimi A, Adoui L, Kollmus H, Dörner R, Weber Th, Khayyat K, Mann R, Grandin J P, and Ullrich J, 1999 J. Phys. B 321859

Bapat B, Keller S, Moshammer R, Mann R, Ullrich J 2000 J. Phys. B 331437

Bastos de Jesus V et al 2003 Phys. Rev. Lett. (submitted)

Bates D R and Griffing G 1953 Phys. Soc. London A 66961

Bates D R and Griffing G 1954 Phys. Soc. London A 67663

Bates D R and Griffing G 1955 Phys. Soc. London A 6890

Becker A and Faisal F H M 2000 Phys. Rev. Lett. 843546

Becker A and Faisal F H M 2002 Phys. Rev. Lett. 89193003

Bethe H 1930 Ann. Phys. Lpz. 5325

Berakdar J and Klar H 1992 Phys. Rev. Lett. 691175 
Berakdar J, Klar H, Huetz A and Selles P 1993 J. Phys. B 261463

Berakdar J 1998 J. Phys . B 313167

Berakdar J 1999 J. Phys. B 32 L25

Berakdar J and Klar H 2001 Phys. Rep. 340474

Bernardi G C, Suárez S, Fainstein P D, Garibotti C R, Meckbach W, Focke P 1989 Phys. Rev. A 406863

Bernardi G, Fainstein P, Garibotti C R, Suarez S 1990 J. Phys. B 23 L139

Brabec T and Krausz F, Rev. Mod. Phys. 200072545

Bray I 2002 Phys. Rev. Lett. 89 273201-1

Bräuning H P, Dörner R, Cocke C L, Prior M H, Krässig B, Bräuning-Demian A, Carnes K, Dreuil S, Mergel V, Richard P, Ullrich J and Schmidt-Böcking H 1997 J. Phys. B 30 L649

Bräuning $\mathrm{H} \mathrm{P}$, Dörner $\mathrm{R}$, Cocke $\mathrm{C}$ L, Prior $\mathrm{M} \mathrm{H}$, Krässig $\mathrm{B}$, Kheifets A, Bray I, Bräuning-Demian A, Carnes K, Dreuil S, Mergel V, Richard P, Ullrich J and Schmidt-Böcking H 1998 J. Phys. B 315149

Briggs J S and Schmidt V 2000 J. Phys. B 33 R1

Bronk T, Reading J F and Ford A L 1998 J. Phys. B 312477

Buchet-Poulizak M-C, Bogdanovich P O and Knystautas É 2001 J. Phys. B 34233

Chassid M, Horbatsch M 2002 Phys. Rev. A 6612714

Cassimi A, Duponchel S, Flechard X, Jardin P, Sortais P, Hennecart D and Olson R E 1996 Phys. Rev. Lett. 763697

Chen J et al Phys. Rev. 200063 011404R

Chen J and Nam C H 2002 Phys. Rev. A 66053415

Cherepkov N A, Raseev G, Adachi J, Hikosaka Y, Ito K, Motoki S, Sano M, Soejima K and Yagishita A 2000 J. Phys. 334213

Cherepkov N A, Semenov S K, Hikosaka Y, Ito K, Motoki S and Yagishita A 2000a Phys. Rev. Lett. 84250

Cocke C L and Olson R E 1991 Phys. Rep. 205155

Choi H C, Rao R M, Mihill A G, Kakar S, Poliakoff E D, Wang K and McKoy V 1994 Phys. Rev. Lett. 7244

Colgan J, Pindzola M S and Robicheaux F 2001 J. Phys. 34 L457

Colgan J and Pindzola M S 2002 Phys. Rev. A 65032729

Collins S A, Cvejanovic S, Dawson C, Reddish T, Seccombe D P, Huetz A, Malegat L, Selles P, Kazansky A K, Danjo A, Soejima K, Okuno K and Yagishita A 2002 Phys. Rev. A $\mathbf{6 5} 052717$

Coplan M A et al. 1994 Rev. Mod. Phys. 66985 and references therein

Corkum B P, Burnett N H and Brunel F 1989 Phys. Rev. Lett. 621259

Corkum P 1993 Phys. Rev. Lett. 711994

Crothers D S F and McCann J F 1983 J. Phys. B16 3229 
Dalitz R H 1953 Phil. Mag. 441068

Davies J A, Continetti R E, Chandler D W and Hayden C C 2000 Phys. Rev. Lett. 845983

De Fanis A, Saito N, Pavlychev A A, Ladonin D Yu, Machida M, Kubozuka K, Koyano I, Okada K, Ikejiri K, Cassimi A, Czasch A, Dörner R, Chiba H, Sato Y and Ueda K 2002 Phys. Rev. Lett. $\mathbf{8 8} 073002$

DeHaven W R, Dilley C, Landers A, Kamber EY and Cocke C L 1998 Phys. Rev. A 57292

Dehmer J L and Dill D 1976 J. Chem. Phys. 655327

Dill D 1976 J. Chem. Phys, 651130

Dill D, Swanson J R, Wallace S and Dehmer J L 1980 Phys. Rev. Lett. 451393

Delone N B and Krainov V P J. 1991 Opt. Soc. Am. B 81207

Delone N B and Krainov V P 1998 Phys. Usp. 41 (5) 469

Dietrich P et al 1994 Phys. Rev. A 50 R3585

DiMauro L and Agostini P 1995 Advances in Atomic and Molecular Physics (Academic Press), New York

Dogan M and Crowe A 2002 J. Phys. B 352773

Dorn A, Moshammer R, Schröter C S, Zouros T J M, Schmitt W, Kollmus H, Mann R and Ullrich J 1999 Phys. Rev. Lett. 822496.

Dorn A, Kheifets A, Schröter C D, Najjari B, Höhr C, Moshammer R and Ullrich J 2001 Phys. Rev. Lett. 863755

Dorn A., Kheifets A., Schröter C D, Najjari B, Höhr C, Moshammer R and Ullrich J 2002 Phys. Rev. A 652709

Dorn A, Sakhelashvili G, Höhr C, Kheifets A, Lower J, Najjari B, Schröter C D, Moshammer $\mathrm{R}$ and Ullrich $\mathrm{J}$ 2002a in Electron and Photon Impact Ionisation and Related Topics 2002: Proceedings of the International Conference on Electron and Photon Impact Ionisation and Related Topics, Metz, France, 18 to 20 July 2002 edtited by L. U. Ancarani, IOP Publishing

Dorn A, Kheifets A, Schröter C D, Höhr C, Sakhelashvili G, Moshammer R, Lower J and Ullrich J 2003 Phys. Rev. A. submitted

Dörner R, Mergel V, Ali R, Buck U, Cocke C L, Froschauer K, Jagutzki O, Lencinas L, Meyerhof W E, Nüttgens S, Olson R E, Schmidt-Böcking H, Spielberger L, Tökesi K, Ullrich J, Unverzagt M and Wu W 1994 Phys. Rev. Lett. 723166

Dörner R, Mergel V, Zhaoyuan L, Ullrich J, Spielberger L, Olson R.E, Schmidt-Böcking H 1995 J. Phys. B 28435

Dörner R, Vogt T, Mergel V, Khemliche H, Kravis S, Cocke C L, Ullrich J, Unverzagt M, Spielberger L, Damrau M, Jagutzki O, Ali I, Weaver B, Ullmann K, Hsu C C, Jung M, Kanter E P, Sonntag B, Prior M H, Rotenberg E, Denlinger J, Warwick T, Manson S T, and Schmidt-Böcking H 1996 Phys. Rev. Lett. 762654

Dörner R, Khemliche H, Prior M H, Cocke C L, Gary J A, Olson R E, Mergel V, Ullrich J and Schmidt-Böcking H 1996a Phys. Rev. Lett. 774520

Dörner R, Feagin J M, Cocke C L, Bräuning H, Jagutzki O, Jung M, Kanter E P, Khemliche H, Kravis S, Mergel V, Prior M H, Schmidt-Böcking H, Spielberger L, Ullrich J, Un- 
verzagt M and Vogt T 1996b Phys. Rev. Lett. 771024 see erratum in Phys. Rev. Lett. 1997782031

Dörner R, Mergel V, Spielberger L, Achler M, Khayyat Kh, Vogt T, Bräuning H, Jagutzki O, Weber T, Ullrich J, Moshammer R, Unverzagt M, Schmitt W, Khemliche H, Prior M H, Cocke C L, Feagin J, Olson R E and Schmidt-Böcking H 1997 Nucl. Instr. Meth. B 124 225

Dörner R, Bräuning H, Feagin J M, Mergel V, Jagutzki O, Spielberger L, Vogt T, Khemliche H, Prior M H, Ullrich J, Cocke C L and Schmidt-Böcking H 1998 Phys. Rev. A 571074

Dörner R, Bräuning H, Jagutzki O, Mergel V, Achler M, Moshammer R, Feagin J M, Osipov T, Bräuning-Demian A, Spielberger L, McGuire J H, Prior M H, Berrah N, Bozek J D, Cocke C L and Schmidt-Böcking H 1998a Phys. Rev. Lett 815776

Dörner R, Mergel V, Bräuning H, Achler M, Weber T, Khayyat Kh, Jagutzki O, Spielberger L, Ullrich J, Moshammer R, Azuma Y, Prior M H, Cocke C L and Schmidt-Böcking H 1998b Atomic processes in Plasmas, AIP conference proceedings 443 ed.: E. Oks, M. Pindzola.

Dörner R, Mergel V, Jagutzki O, Spielberger L, Ullrich J, Moshammer R and SchmidtBöcking H 2000 Physics Reports 33095

Dörner R, Mergel V, Jagutzki O, Spielberger L, Ullrich J, Moshammer R and SchmidtBöcking H 2002 Adv. in Atom., Mol., and Opt. Physics 48

Drescher M 2001 et al Science 2911923

Drescher M 2002 et al Nature 419803

DuBois R D, Ali I, Cocke C L, Feeler C R and Olson R E 2000 Phys. Rev. A 62060701

Dupré C, Lahmam-Bennani A, Duguet A, Mota-Furtado F, O’Mahony P F and Capello C D 1992 J. Phys. B 25259

Edgü-Fry E, Cocke C L, Sidky E, Lin C D and Abdallah M 2002 J. Phys. B 352603

Ehrhardt H., Schulz M., Tekaat T. and Willmann K 1969 Phys. Rev. Lett. 2289

Ehlotsky F, Jaron A and Kaminski J Z 1998 Phys. Rep. 2976

Eichmann U et al 2000 Phys. Rev. Lett. 843550

Eremina E, Rottke H, Sandner W, Dreischuh A, Lindner F, Grasbon F, Paulus G G, Walther H, Moshammer R, Feuerstein B and Ullrich J 2003 J. Phys. B (submitted)

Fainstein P D, Ponce V H and Rivarola R D 1988 J. Phys. B 21287

Fainstein P, Gulyás L, Martín F and Salin A 1996 Phys. Rev. A 533243

Fainstein P D, Moshammer R and Ullrich 2001 J Phys. Rev. A 63062720

Faisal F H M 1973 J. Phys. B 6 L89

Feagin J M and Briggs J S 1986 Phys. Rev. Lett. 57984

Feagin J M 1995 J. Phys. B 281495

Feagin J M 1996 J. Phys. B 291551

Feagin J M 1998 J. Phys. B 31 L729

Feeler C R and Olson R E J. Phys. B 331997 (2000) 
Feeler C R, Olson R E, DuBois R D, Schlathölter T, Hadjar O, Hoekstra R and Morgenstern R 1999 Phys. Rev. A 602112

Feuerstein B, Moshammer R and Ullrich J 2000 J. Phys. B 33 L823

Feuerstein B Moshammer R, Fischer D, Dorn A, Schröter C D, Deipenwisch J, Crespo LopezUrrutia J R, Höhr C, P. Neumayer, Ullrich J, Rottke H, Trump C, Wittmann M, Korn G and Sandner W 2001 Phys. Rev. Lett. 87043003

Feuerstein B, Schulz M, Moshammer R and Ullrich J 2001a Physica Scripta T92 447

Fiol J, Olson R E, Santos A C F, Sigaud G M and Montenegro E C 2001 J. Phys. B 34 L503

Fischer D, Feuerstein B, Dubois R D, Moshammer R, Crespo López-Urrutia J R, Draganic I, Lörch H, Perumal A N and Ullrich J 2002 J. Phys. B 351369

Fischer D, Moshammer R, Dorn A, Crespo López-Urrutia J R, Feuerstein B, Höhr C, Schröter C D, Hagmann S, Kollmus H, Mann R, Bapat B and Ullrich J 2003 Phys. Rev. Lett. accepted

Fischer D, Moshammer R, Schulz M, Voitkiv A, Madison D H, Foster M and Ullrich J 2003a J. Phys. B submitted

Fischer D, Voitkiv A B, Moshammer R and Ullrich J 2003 Phys. Rev. A submitted

Flechard X, Nguyen H, Wells E, Ben-Itzhak I and DePaola B D 2001 Phys. Rev. Lett. 87 123203

Flechard X, Harel C, Jouin H, Pons B, Adoui L, Fremont F, Cassimi A and Hennecart D 2001a J. Phys. B 342759

Fittinghoff D N et al 1992 Phys. Rev. Lett. 692642

Fittinghoff D N 1994 et al Phys. Rev. A 49, 2174

Ford A L and Reading J F 1988 J. Phys. B 21 L685

Ford A L and Reading J F 1990 J. Phys. B 232567

Frémont F, Guillaume L, Rangama J, Sobocinski P, Tarisien M, Adoui L, Cassimi A, Chesnel J Y, Fléchard X, Hennecart D and Husson X 2002 Int. J. Mol. Sci. 3115

Fritsch W 1994 J. Phys. B 273461

Gay T J, Gealy M W, Rudd M E 1990 J. Phys. B 23 L823

Gensmantel A, Ullrich J, Dörner R, Olson R E, Ullmann K, Forberich E, Lencinas S and Schmidt-Böcking H 1992 Phys. Rev. A 454572

Gerchikov L G and Sheinermann S A 2001 J. Phys. B 34647

Gerchikov L G, Sheinermann S A, Schulz M, Moshammer R and Ullrich J 2002 J. Phys. B 35 2783

Gessner O, Hikosaka Y, Zimmermann B, Hempelmann A, Lucchese R, Eland J, Guyon P and Becker U 2002 Phys. Rev. 88193002

Golovin A V, Heiser F, Quayle C J K, Morin P, Simon M, Gessner O, Guyon P M and Becker U 1997 Phys. Rev. Lett. 794554

Goreslavskii S and Popruzhenko S 2001 Optics Express 8395

Grin M, Dal Cappello C, Mkhanter R El and Rasch J 2000 J. Phys. B 33131 
Guillemin R, Shigemasa E, Le Guen K, Ceolin D, Miron C, Leclercq N, Morin P and M. Simon M 2001 Phys. Rev. Lett. 87203001

Gulyás L, Kirchner T, Shirai T and Horbatsch M 2000 Phys. Rev. A 62022702

Illescas C, Rabadán L, Riera A 1998 Phys. Rev. A 571809

Irby V D, Gay T J, Edwards J Wm, Hale E B, McKenzie M L, Olson R E 1988 Rev. A 37 3612

Hasan A A, Emmons E D, Hinojosa G and Ali R 1999 Phys. Rev. Lett. 834522

Hasegawa H, Hishikawa A and Yamanouchi K 2001 Chem. Phys. Lett. 34957

Hattass M 2003, private communication

Hayes P A and Williams J F 1996 Phys. Rev. Lett. 773098

Heiser F, Gessner O, Viefhaus J, Wieliczec K, Hentges R and Becker U 1997 Phys. Rev. Lett. 792435

Helm H, Bjerre N, Dyer M J, Huestis D L, and Saeed M 1993 Phys. Rev. Lett. 703221

Hikosaka Y and Eland J 2000 J. Phys. B 333137

Hikosaka Y and Eland J 2000a Chem. Phys. 204663

Hikosaka Y, Eland J, Watson T and Powis I 2001 J. Chem. Phys. 1154593

Hikosaka Y and Eland J 2002 Chem. Phys. 27753

Hippler R, Datz S, Miller P D, Pepmiller P L and Dittner P F 1987 Phys. Rev. A 35585

Huetz A, Selles P, Waymel D and Mazeau J 1991 J. Phys., B 241917

Huetz A and Mazeau J 2000 Phys. Rev. Lett. 85530

Ito K, Adachi J, Hall R, Motoki S, Shigemasa E, Soejima K and Yagishita A 2000 J. Phys. 33 572

Ito K, Adachi J, Hikosaka Y, Motoki S, Soejima K, Yagishita A, Raseev G and Cherepkov N A 2000a Phys. Rev. Lett. 8546

Jagutzki O, Spielberger L, Dörner R, Nüttgens S, Mergel V, Schmidt-Böcking H, Ullrich J, Olson R E and Buck U 1996 Z. Phys. D 365

Jagutzki O, Mergel V, Ullmann-Pfleger K, Spielberger L, Meyer U, Dörner R, SchmidtBöcking H in: Imaging Spectroscopy IV, Proceedings of International Symposium on Optical Science Engineering and Instrumentation, 1998 Proc. SPIE Vol 3438, pp 322333, Eds. M. R. Descour, S.S. Shen

Jagutzki O, Cerezo A, Czasch A, Dörner R, Hattaß M, Huang M, Mergel V, Spillmann U, Ullmann-Pfleger K, Weber T, Schmidt-Böcking H, Smith G D W 2002 IEEE Transact. on Nucl. Science, Vol. 49 No. 5, 2477

Jagutzki O, Lapington J S, Worth L B C, Spillmann U, Mergel V, Schmidt-Böcking H 2002a Nucl. Instr. Meth. in Phys. Res. A 477256

Jagutzki O, Mergel V, Ullmann-Pfleger K, Spielberger L, Spillmann U, Dörner R, SchmidtBöcking H 2002b Nucl. Instr. and Meth. in Phys. Res. A 477244

Jahnke T, Weber Th, Landers A L, Knapp A, Schössler S, Nickles J, Kammer S, Jagutzki O, Schmidt L, Czasch A, Osipov T, Arenholz E, Young A T, Diez Muino R, Rolles D, Garcia de Abajo F J, Fadley C S, Van Hove M A, Semenov S K, Cherepkov N A, 
Rösch J, Prior M H, Schmidt-Böcking H, Cocke C L and Dörner R 2002 Phys. Rev. Lett. 88073002

Jahnke T 2003 private communication

Jalowy T, Neugebauer R, Hattass M, Fiol J, Afaneh F, Pereira J A M, Collado V, da Silveira E F, Schmidt-Böcking H and Groeneveld K O 2002 Nucl. Instr. Meth. B 193762

Jalowy T, Neugebauer R, Groeneveld K O, Ponciano C R, Farenzena L S, da Silveira E F 2002a Int. J. Mass Spectrom. 219343

Jalowy T, Neugebauer R, Groeneveld K O, Ponciano C R, Farenzena L S, da Silveira E F 2002b Rev. Scientific Instrum. 733187.

Jalowy T, Neugebauer R, Farenzena L S, Collado V M, Schmidt-Böcking H, da Silveira E F, Groeneveld K O 2003 Proceedings of the $17^{\text {th }}$ CAARI Conference (November 12-16, 2002, Denton, USA) (to appear)

Jalowy T, Weber Th, Dörner R, Farenzena L S, Collado V M, Schmidt-Böcking H, da Silveira E F, Groeneveld K O 2003a Int. J. of Mass Spectrometry (submitted to)

Jardin P, Cassimi A, Grandin J P, Rothard H, Lemoigne J P, Hennecart D, Husson X and Lepontre A 1996 Nucl. Instrum. Method. B 10741

Kamber E Y, Abdallah M A, Cocke C L, Stöckli M, Wang J and Hansen J P 2000 J. Phys. B 33 L171

Kazanski A K and Ostrovsky V N 1993 Phys. Rev. A 48 R871

Kazanski A K and Ostrovsky V N 1994 J. Phys. B 27447

Kazanski A K and Ostrovsky V N 1995 Phys. Rev. A 513698

Keldysh L V 1964 Zh. Eksp. Teor. Fiz. 471945

Keller S, Lüdde H J and Dreizler R M 1997 Phys. Rev. A 554215

Keller S 2000 J. Phys. B 33 L513

Keller S, Bapat B, Moshammer R, Ullrich J, Dreizler R M 2000a J. Phys. B 331447

Khalil D, Maquet A, Taïeb R, Joachain C J and Makhoute A 1997 PRA 56, 4918

Khayyat Kh, Weber Th, Dörner R, Achler M, Mergel V, Spielberger L, Jagutzki O, Meyer U, Ullrich J, Moshammer R, Schmitt W, Knudsen H, Mikkelsen U, Aggerholm P, Uggerhoej E, Moeller S P, Rodríguez V D, O’Rourke S F C, Olson R E, Fainstein P D, McGuire J H and Schmidt-Böcking H 1999 J. Phys. B 32 L73

Kheifets A and Bray I 1998 Phys. Rev. Lett. 814588

Kheifets A and Bray I 1998a J. Phys. B 31 L447

Kheifets A S et al 1999 J. Phys. B 325047

Kheifets A, Bray I, Soejima K, Danjo A, Okuno K and Yagishita A 1999a J. Phys. B 32 L501

Kheifets A and Bray I 2000 Phys. Rev. A 62065402

Kheifets A 2003 private communication

Kirchner T 2002 Phys. Rev. Lett. 89093203

Kirchner T, Gulyás L, Schulz M, Moshammer R and Ullrich J 2002a Phys. Rev. A 65042727 
Knapp A, Kheifets A, Bray I, Weber Th, Landers A L, Schössler S, Jahnke T, Nickles J, Kammer S, Jagutzki O, Schmidt L, Osipov T, Rösch J, Prior M H, Schmidt-Böcking H, Cocke C L and Dörner R 2002 Phys. Rev. Lett. 89033004

Knapp A, Walter M, Weber Th, Landers A L, Schössler S, Jahnke T, Schöffler M, Nickles J, Kammer S, Jagutzki O, Schmidt L, Osipov T, Rösch J, Prior M H, Schmidt-Böcking H, Cocke C L, Feagin J and Dörner R 2002a J. Phys. B 35 L521

Kollmus H, Moshammer R, Olson R E, Hagmann S, Schulz M and Ullrich J 2002 Phys. Rev. Lett. 88 103202-1

Kossmann H, Schwarzkopf O, Kämmerling B and Schmidt 1989 V. Phys. Rev. Lett. 632040

Krässig B, Dunford R W, Gemmell D S, Hasegawa S, Kanter E P, Schmidt-Böcking H, Schmitt W, Southworth S H, Weber Th, and Young L 1999 Phys. Rev. Lett. 8353

Kravis S D, Abdallah M, Cocke C L, Lin C D, Stöckli M, Walch B, Wang Y, Olson R E, Rodriguez V D, Wu W, Pieksma M, Watanabe N 1996 Phys. Rev. A 541394

Kuchiev M Y 1987 Sov. Phys.-JET Lett. 45404

Kuznetsov V V and Cherepkov N A 1996 J. Electron Spectrosc. Relat. Phenom. 79437

Lahmam-Bennani A, Dupré C and Duguet A 1989 Phys. Rev. Lett. 631582

Lafosse A, Lebech M, Brenot J C, Guyon P M, Jagutzki O, Spielberger L, Vervloet M, Houver J C and Dowek D 2000 Phys. Rev. Lett. 845987

Lafosse A, Brenot J C, Hoejrup K, Guyon P M, Houver J C, Lebech M and Dowek D 2001 J. Chem. Phys. 1146605

Lafosse A, Brenot J C, Guyon P M, Houver J C, Golovin A V, Lebech M, Dowek D, Lin P and Lucchese R R 2002 J. Chem. Phys. 1178368

Lahmam-Bennani A 1991 J. Phys. B 242401

Lambropoulos P, Maragakis P and Zhang J 1998 Physics Reports 305203

Landers A L, Weber Th, Ali I, Cassimi A, Hattass M, Jagutzki O, Nauert A, Osipov T, Staudte A, Prior M H, Schmidt-Böcking H, Cocke C L and Dörner R 2001 Phys. Rev. Lett. 87013002

Larochelle S, Talebpour A and Chin S L 1998 J. Phys. B 31, 1201

Lee D H, Zouros T J M, Sanders J M, Richard P, Anthony J M, Wang Y D and McGuire J H 1992 Phys. Rev. A 461374

Lee T G, Nguyen H, Flechard X, DePaola B D and Lin C D 2002 Phys. Rev. A 66042701

Lein M, Gross E and Engel V 2000 Phys. Rev. Lett. 854707

Lucey S P, Rasch J, Wheelan C T and Walters H R J 1998 J. Phys. B 311237

Macek J and Ovchinnikov S Yu 1998 Phys. Rev. Lett. 802298

Madison D, Schulz M, Jones S, Foster M, Moshammer R and Ullrich J 2002 J. Phys. B 35 3297

Madison D H, Fischer D, Foster M, Schulz M, Moshammer R, Jones S and Ullrich J 2003 Phys. Rev. Lett. submitted

Makhoute A, Khalil D, Maquet A, Joachain C J, Taieb R 1999 J.Phys B 323255 
Makhoute A, Khalil D, Zitane M and Bouzidi M 2002 J.Phys B 35957

Malegat L, Selles P and Huetz A 1997 J. Phys. B 30251

Malegat L, Selles P and Kazansky A K 2000 Phys. Rev. Lett. 8521

Malegat L, Selles P and Kazansky A K 2002 Phys. Rev. A 65032711

Martin C, Jelinsky P, Lamton M, Malina R F and Anger H O 1981 Rev. Sci. Instrum. 521067

Matthäus R, Moshammer R, Wien K 1991 NATO ASI Series B 26955

Matthäus R, Moshammer R, von Hayn G, Wien K 1993 Int. J. Mass Spectr. and Ion. Proc. 12645

Maulbetsch F and Briggs J S 1995 J. Phys. B 28551

McCarthy I E and Weigold E 1991 Rep. Prog. Phys. 54789

McGuire J H, Stolterfoht N and P R Simony 1981 Phys. Rev. A 2497

McGuire J H, Berrah N, Bartlett R J, Samson J A R, Tanis J A, Cocke C L, and Schlachter A S 1995 J. Phys. B 28913

McGuire J H, Electron Correlation Dynamics in Atomic Collisions 1997 Cambridge monographs on Atomic, Molecular and Chemical Physics, Cambridge: Cambridge University Press

Meckbach W, Suárez S, Focke P, Bernardi G 1991 J. Phys. B 243763

Mergel V, Dörner R, Ullrich J, Jagutzki O, Lencinas S, Nüttgens S, Spielberger L, Unverzagt M, Cocke C L, Olson R E, Schulz M, Buck U, Zanger E, Theisinger W, Isser M, Geis S and Schmidt-Böcking H 1995 Phys. Rev. Lett. 742200

Mergel V, Dörner R, Achler M, Khayyat Kh, Lencinas S, Euler J, Jagutzki O, Nüttgens S, Unverzagt M, Spielberger L, Wu W, Ali R, Ullrich J, Cederquist H, Salin A, Wood C J, Olson R E, Belkic Dz, Cocke C L and Schmidt-Böcking H 1997 Phys. Rev. Lett. 79387

Mergel V, Achler M, Dörner R, Khayyat Kh, Kambara T, Awaya Y, Zoran V, Nyström B, Spielberger L, McGuire J H, Feagin J, Berakdar J, Azuma Y and Schmidt-Böcking H 1998 Phys. Rev. Lett. 805301

Mergel V, Dörner R, Khayyat Kh, Achler M, Weber T, Jagutzki O, Lüdde H J, Cocke C L and Schmidt-Böcking H 2001 Phys. Rev. Lett. 862257

Miller R 1998 in: Atomic and Molecular Beam Methods, Vol. 14, eds.: G. Scoles, D. Bassi, U. Buck, D. Laine, Oxford University Press, New York

Mkhanter R El and Dal Cappello 1998 C J. Phys. B 31301

Montenegro E C and W E Meyerhof 1992 Phys. Rev. A 465506

Montenegro E C, Melo W S, Meyerhof W E and dePinho A G 1992 Phys. Rev. Lett 693033

Montenegro E C, Belkacem A, Spooner D W, Meyerhof W E and Shah M B 1993 Phys. Rev. A 471045

Morgner U et al 2001 Phys. Rev. Lett. 865462

Moshammer R, Matthäus R, Wien K, Bolbach G 1990 Proc. of the $5^{\text {th }}$ Int. Conf. on "Ion Formation from Organic Solids (IFOS 5)" ed. Hedin A Wiley 1990 p 17

Moshammer R, Ullrich J, Unverzagt M, Schmitt W, Jardin P, Olson R E, Mann R, Dörner R, Mergel V, Buck U and Schmidt-Böcking H 1994 Phys. Rev. Lett. 733371 
Moshammer R, Unverzagt M, Schmitt W, Ullrich J and Schmidt-Böcking H 1996 Nucl. Instrum. and Method. B 108425

Moshammer R, Ullrich J, Unverzagt M, Schmitt W, Jardin P, Olson R E, Dörner R, Mergel V and Schmidt-Böcking H 1996a Nucl. Instr. Meth. B 10762

Moshammer R, Ullrich J, Kollmus H, Schmitt W, Unverzagt M, Jagutzki O, Mergel V, Schmidt-Böcking H, Mann R, Woods C J and Olson R E 1996b Phys. Rev. Lett. 77 1242

Moshammer R, Schmitt W, Ullrich J, Kollmus H, Cassimi A, Dörner R, Jagutzki O, Mann R, Olson R E, Prinz H T, Schmidt-Böcking H, Spielberger L 1997 Phys. Rev. Lett. 79 3621

Moshammer R, Ullrich J, Kollmus H, Schmitt W, Unverzagt M, Schmidt-Böcking H, Wood C J and Olson R E 1997a Phys. Rev. A 561351

Moshammer R and Ullrich J 1998 „A Method and Device for Surface Analysis“ German Patent Nr. 19803146.7

Moshammer R, Fainstein P D, Schulz M, Schmitt W, Kollmus H, Mann R, Hagmann S, Ullrich J 1999 Phys. Rev. Lett. 834721

Moshammer R, Feuerstein B, Schmitt W, Dorn A, Schröter C D, Ullrich J, Rottke H, Trump C, Wittmann M, Korn G, Hoffmann K and Sandner W 2000 Phys. Rev. Lett. 84447

Moshammer R, Perumal A, Schulz M, Rodríguez V D, Kollmus H, Mann R, Hagmann S and Ullrich J 2001 Phys. Rev. Lett. 87 223201-1

Moshammer R, Feuerstein B, Crespo Lopez-Urrutia J R, Deipenwisch J, Dorn A, Fischer D, Höhr C, P. Neumayer, Schröter C D, Ullrich J, Rottke H, Trump C, Wittmann M, Korn G and Sandner W 2002 Phys. Rev. A 65035401

Moshammer R, Ullrich J, Feuerstein B, Fischer D, Dorn A, Schröter C D, Crespo LopezUrrutia J R, Höhr C, Rottke H, Trump C, Wittmann M, Korn G, Hoffmann K and Sandner W 2003 Phys. Rev. A (in print)

Moshammer R, Ullrich J, Feuerstein B, Fischer D, Dorn A, Schröter C D, Crespo LópezUrrutia J R, Höhr C, Rottke H, Trump C, Wittmann M, Korn G and Sandner W 2003a Phys. Rev. Lett. (submitted)

Moshammer R, Kollmus H and Fischer D 2003b in: Many Particle Quantum Dynamics in Atomic Fragmentation eds.: Ullrich J and Shevelko S, Springer -Verlag Berlin (in press)

Motoki S, Adachi J, Ito K, Ishii K, Soejima K, Yagishita A, Semenov S K and Cherepkov N A 2002 Phys. Rev. Lett. 88063003

Muramatsu Y, Ueda K, Saito N, Chiba H, Lavolle M, Czasch A, Weber T, Jagutzki O, Schmidt-Böcking H, Moshammer R, Becker U, Kubozuka K and Koyano I 2002 Phys. Rev. Lett. 88133002

Niehaus A 1986 J. Phys. B 192925

Olson R E 1983 Phys. Rev A 271871

Olson R E 1986 Phys. Rev A 334397

Olson R E, Gay T J, Berry H G, Hale E B, Irby V D 1987 Phys. Rev. Lett. 5936

Olson R E, Ullrich J, Schmidt-Böcking H 1989 Phys. Rev. A 39, 5572 
Olson R E, Feeler CR, Wood C J, Cocke C L, Dörner R, Mergel V, Schmidt-Böcking H 1997 Nucl. Instr. Meth. B 124, 249

Olson R E, Wood C J, Schmidt-Böcking H, Moshammer R and Ullrich J 1998 Phys. Rev. A 58270

Olson R E and Fiol J 2001 J. Phys. B 34 L625

Olson R E, Fiol J, Moshammer R and Ullrich J 2003 J. Phys. B 36 L99

O’Rourke S F C, Moshammer R and Ullrich J 1997 J. Phys. B 305281

Osipov T, Cocke C L, Prior M H, Weber T, Jagutzki O, Schmidt L, Schmidt-Böcking H, Dörner R and Landers A 2003 Phys. Rev. Lett. accepted for publication

Ovchinnikov S Yu, Macek J H 1995 Phys. Ref Lett 752474

Parker J S et al 2001 J. Phys. B 34 L691

Pavlychev A A, Fominykh N G, Watanabe N, Soejima K, Shigemasa E and Yagishita A 1998 Phys. Rev. Lett. 813623

PHELIX 2001 http://www-aix.gsi.de/ phelix/

Pieksma M, Ovchinnikov S Yu 1991 J. Phys. B 242699

Pieksma M, Ovchinnikov S Yu 1994 J. Phys. B 274573

Pieksma M, Ovchinnikov S Y, van Eck J, Westerveld W B, Niehaus A 1994 Phys. Rev. Lett. 73, 46

Perumal A N, Moshammer R, Schulz M and Ullrich J 2002 J. Phys. B 352133

Pont M and Shakeshaft R 1995 Phys. Rev. A 51 R2676

Pont M, Shakeshaft R, Maulbetsch F and Briggs J S 1996 Phys. Rev. A 533671

Pont M and Shakeshaft R 1996a Phys. Rev. A 541448

Poon H C and Tong S Y 1984 Phys. Rev. B 306211

Protopapas M, Keitel C and Knight P 1997 Physics Reports 60389

Reddish T J, Wightman J P, MacDonald M A and Cvejanovic S 1997 Phys. Rev. Lett. 79 2438

Reddish T J and Feagin J 1998 J. Phys. B 322473

Reiss H R 1980 Phys. Rev. A 221786

Reiss H R 1987 J. Phys. B 20 L79

Rescigno T N et al 1999 Science 2862474

Rottke H 2002 et al Phys. Rev. Lett. 89 013001-1

Rudd M E, Kim Y-K, Madison D H and Gay T J 1992 Rev. Mod. Phys. 64441

Saito N, De Fanis A, Kubozuka K, Machida M, Takahashi M, Yoshida H, Suzuki I H, Cassimi A, Czasch A, Schmidt L, Dörner R, Wang K, Zimmermann B, McKoy V, Koyano I and Ueda K 2003 J. Phys. B 36 L24

Samson J A R, He Z X, Bartlett R J and Sagurton M 1994 Phys. Rev. Lett. 723329

Schmidt-Böcking H, Mergel V, Dörner R, Jagutzki O, Schmidt L, Weber T, Cocke C L, Lüdde H J, Weigold E, Popov Yu V, Cederquist H, Schmidt H T, Schuch R, Berakdar J 2002 in: AIP Conference Proceedings 604, ed: Madison D H and Schulz M page 120 
Schmidt-Böcking H, Dörner R, Ullrich J 2002a Euro Physics News 6210

Schmidt-Böcking H, Mergel V, Dörner R, Cocke C L, Jagutzki O, Schmidt L, Weber Th, Lüdde H J, Weigold E, Berakdar J, Cederquist H, Schmidt H T, Schuch R, Kheifets A S 2003 Europhys. Lett. 62477

Schmidt-Böcking H, Mergel V, Dörner R, Schmidt L, Weber T, Weigold E, Kheifets A S 2003a in: Many-Particle Quantum Dynamics in Atomic and Molecular Fragmentation, e.d. Ullrich J and Shevelko V P, Springer Verlag in print

Schmidt H T, Fardi A, Schuch R, Schwartz S H, Zettergren H, Cederquist H, Bagge L, Danared H, Källberg A, Jensen J, Rensfelt K-G, Mergel V, Schmidt L, SchmidtBöcking H, and Cocke C L 2002 Phys. Rev. Lett. 89 163201-1

Schmidt L 2000 PhD-thesis, Johann-Wolfgang-Goethe Universität Frankfurt

Schmidt W, Moshammer R, O’Rourke F S C, Kollmus H, Sarkadi L, Mann R, Hagmann S, Olson R E, Ullrich J 1998 Phys. Rev. Lett. 814337

Schöne H, Schuch R, Datz S, Schulz M, Dittner P F, Giese J P, Kessel Q C, Krause H F, Miller P D and Vane C R 1995 Phys. Rev. A 51324

Schwarzkopf O, Krässig B, Elmiger J and Schmidt V 1993 Phys. Rev. Lett. 703008

Schwarzkopf O and Schmidt V 1995 J. Phys. B 282847

Schuch R, Schöne H, Miller P D, Krause H F, Dittner P F, Datz S and Olson R E 1988 Phys. Rev. Lett. 60925

Schultz D R, Reinhold C O, Krstic P S, Strayer M R 2002 Phys Rev. A 6552722

Schulz M, Moshammer R, Schmitt W, Kollmus H, Mann R, Hagmann S, Olson R E and Ullrich J 1999 J. Phys. B 32 L557

Schulz M, Moshammer R, Schmitt W, Kollmus H, Mann R, Hagmann S, Olson R E and Ullrich J 2000 Phys. Rev. A 61022703

Schulz M, Moshammer R, Schmitt W, Kollmus H, Feuerstein B, Mann R, Hagmann S and Ullrich J 2000a Phys. Rev. Lett. 84863

Schulz M, Moshammer R, Madison D H, Olson R E, Marchalant P, Whelan C T, Walters H R J , Jones S, Foster M, Kollmus H, Cassimi A and Ullrich J 2001 J. Phys. B 34 L305

Schulz M, Moshammer R, Gerchikov L G, Sheinermann S A and Ullrich J 2001a J. Phys. B 34 L 795

Schulz M, Moshammer R, Perumal A N and Ullrich J 2002 J. Phys. B 35 L161

Schulz M, Moshammer R, Fischer D, Kollmus H, Madison D H, Jones S and Ullrich J 2003 Nature $\mathbf{4 2 2} 48$

Selles P, Malegat L and Kazansky A K 2002 Phys. Rev. A 65032711

Sheehy B et al 1998 Phys. Rev. A 583942

Shigemasa E, Adachi J, Oura M, and Yagishita A 1995 Phys. Rev. Lett. 74359

Shigemasa E, Adachi J, Soejima K, Watanabe N, Yagishita A and Cherepkov N A 1998 Phys. Rev. Lett. 801622

Sidky E Y, Lin C D 1998 J. Phys. B 312949

Sidky E Y, Lin C D 1999 Phys. Rev. A 60377

Sidky E Y, Illescas C, Lin C D 2000 Phys. Rev. Lett. 851634 
Sidky E Y and Lin C D 2001 Phys. Rev. A 65012711

Sidorovich V A and Nikolaev V S 1983 J. Phys. B 163243

Sobottka S E, Williams M B 1988 IEEE Trans. Nulc. Sci. 35348

Soejima K, Danjo A, Okuno K and Yagishita A 1999 Phys. Rev. Lett. 831546

Solov'ev E A 1986 Sov. Phys. JETP 63678

Spielberger L, Jagutzki O, Dörner R, Ullrich J, Meyer U, Mergel V, Unverzagt M, Damrau M, Vogt T, Ali I , Khayyat Kh, Bahr D, Schmidt H-G, Frahm R and Schmidt-Böcking H 1995 Phys. Rev. Lett. 744615

Spielberger L, Jagutzki O, Krässig B, Meyer U, Khayyat Kh, Mergel V, Tschentscher Th, Buslaps Th, Bräuning H, Dörner R, Vogt T, Achler M, Ullrich J, Gemmell D S and Schmidt-Böcking H 1996 Phys. Rev. Lett. 764685

Spielberger L, Bräuning H, Muthig A, Tang J Z, Wang J, Qui Y, Dörner R, Jagutzki O, Tschentscher Th, Honkimäki V, Mergel V, Achler M, Weber Th, Khayyat Kh, Burgdörfer J, McGuire J and Schmidt-Böcking H 1999 Phys. Rev. A 59371

Staudte A, Cocke C L, Prior M H, Belcacem A, Ray C, chong H W, Glover T E, Schoenlein R W and Saalmann U 2002 Phys. Rev. A 65 020703(R)

Stefani G, Avaldi L and Camilloni R 1990 J. Phys. B 23 L227

Stolterfoht N, Havener C C, Phaneuf R A, Swenson J K, Shafroth S M and Meyer F W 1986 Phys. Rev. Lett. 5774

Stolterfoht N, DuBois R D and Rivarola R D 1994 Electron Emission in Heavy Ion-Atom Collisions, Springer

Stolterfoht N, Chesnel J Y, Grether M, Tanis J A, Skogvall B, Frémont F, Lecler D, Hennecart D, Husson X, Grandin J P, Koncz Cs, Gulyás L, and Sulik B 1999 Phys. Rev. A 591262

Taouil I, Lahmam-Bennani A, Duguet A and Avaldi L 1998 Phys. Rev. Lett. 814600

Tarisien M, Adoui L, Frémont F, Lelièvre D, Guillaume L, Chesnel J Y, Zhang H, Dubois A, Mathur D, Sanjay Kumar, Krishnamurthy M and Cassimi A 2000 J. Phys. B 33 L11

Thomas L H, 1927 Proc. R. Soc. London A 114561

Teng Z J and Shakeshaft R 1994 Phys. Rev. A 493597

Turkstra J W, Hoekstra R, Knoop S, Meyer D, Morgenstern R and Olson R E 2001 Phys. Rev. Lett. 87123202

Ueda K, De Fanis A, Saito N, Machida M, Kubozuka K, Chiba H, Muramatu Y, Sato Y, Czasch A, Jagutzki O, Dörner R, Cassimi A, Kitajima M, Furuta T, Tanaka H, Sorensen S L, Okada K, Tanimoto S, Ikejiri K, Tamenori Y, Ohashi H and Koyano I 2003 Chem. Phys. 289135

Ullrich J 1987 PhD-thesis, Johann-Wolfgang-Goethe Universität Frankfurt

Ullrich J and Schmidt-Böcking H 1987 Phys. Lett. A 125193

Ullrich J, Horbatsch M, Dangendorf V, Kelbch S and Schmidt-Böcking H 1988 J. Phys. B 21 611

Ullrich J, Schmidt-Böcking H and Kelbch C 1988a Nucl. Instr. Meth. A 268216 
Ullrich J, Olson R E, Dörner R, Kelbch S, Berg H and Schmidt-Böcking H 1989 J. Phys. B 22 627

Ullrich J, Moshammer R, Berg H, Mann R, Tawara H, Dörner R, Euler J, Schmidt-Böcking H, Hagmann S, Cocke C L, Unverzagt M, Lencinas S and Mergel V 1993 Phys. Rev. Lett. 711697

Ullrich J, Dörner R, Mergel V, Jagutzki O, Spielberger L and Schmidt-Böcking H 1994 Comments At. Mol. Phys. 30285

Ullrich J 1994 Habilitationsschrift, Universität Frankfurt, GSI Report (1994)-08, ISSN 01714546

Ullrich J, Moshammer R, Unverzagt M, Schmitt W, Jardin P, Olson R E, Dörner R, Mergel V and Schmidt-Böcking H 1995 Nucl. Instr. Meth. B 98375

Ullrich J, Moshammer R, Dörner R, Jagutzki O, Mergel V, Schmidt-Böcking H, Spielberger L 1997 J. Phys. B 302917 Topical Review

Ullrich J, Bapat B, Dorn A, Keller S, Kollmus H, Mann R, Moshammer R, Olson R E, Schmitt W and Schulz M 2000 in X-Ray and Inner Shell Processes, edited by Dunford R W, Gambol D S, Kanter E P, Young L 403-417

Ullrich J and Voitkiv A 2002 in Strong Field Laser Physics edited by Brabec Th and Kapteyn $\mathrm{H}$ Springer (in press)

Ullrich J and Shevelko S 2003 Many Particle Quantum Dynamics in Atomic Fragmentation Springer-Verlag, Berlin (in press)

Ullrich J, Dörner R, Moshammer R, Rottke H, Sandner W 2003a Proceedings of the XVIII International Conference on Atomic Physics edited by Sadeghpour H R, Heller E J and Pritchard D E, World Scientific, 219

Unverzagt M, Moshammer R, Schmitt W, Olson R E, Jardin P, Mergel V, Ullrich J and Schmidt-Böcking H 1996 Phys. Rev. Lett. 761043

Van der Poel M, Nielsen C V, Gearba M A and Andersen N 2001 Phys. Rev. Lett. 87123201

Viefhaus J, Avaldi L, Snell G, Wiedenhöft M, Hentges R, Rüdel A, Schäfer F, Menke D, Heinzmann U, Engelns A, Berakdar J, Klar H and Becker U 1996 Phys. Rev. Lett. 77 3975

Voitkiv A B 1996 J. Phys. B 295433

Voitkiv A B, Grün N and Scheid W 1999 J. Phys. B 323929

Voitkiv A B and Ullrich J 2001 J. Phys. B 344513

Voitkiv A B and Ullrich J 2001a J. Phys. B 341673

Voitkiv A B and Ullrich J 2001b J. Phys. B 344383

Voitkiv A B, Najjari B, Moshammer R and Ullrich J 2002 Phys. Rev. A 65 032707-1

Voitkiv A B, Najjari B, Moshammer R and Ullrich J 2002a J. Phys. B 65032707

Voitkiv A B, Najjari B and Ullrich J 2003 Phys. Rev. A accepted

Wabnitz $\mathrm{H}$ et al 2002 Nature 420482

Walker B et al 1996 Phys. Rev. Lett. 775031

Walter M and Briggs J S 1999 J. Phys. B 322487

Walter M and Briggs J S 2000 Phys. Rev. Lett. 851630 
Wannier G H 1953 Phys. Rev. 90817

Watanabe N, Adachi J, Soejima K, Shigemasa E and Yagishita A 1997 Phys. Rev. Lett. 78 4910

Weber T 1998 Diploma Thesis, Universität Frankfurt, unpublished http://hsbpcl.ikf.physik. uni-frankfurt.de/publications/files/ThorstenWeber-1999 $2^{\text {nd }}$ Edition.pdf

Weber T, Giessen H, Weckenbrock M, Staudte A, Spielberger L, Jagutzki O, Mergel V, Urbasch G, Vollmer M and Dörner R et al 2000 Phys. Rev. Lett. 84443

Weber T, Weckenbrock M, Staudte A, Spielberger L, Jagutzki O, Mergel V, Urbasch G, Vollmer M, Giessen H and Dörner R 2000a J. Phys. B 33 L127

Weber T, Weckenbrock M, Staudte A, Spielberger L, Jagutzki O, Mergel V, Urbasch G, Vollmer M, Giessen H and Dörner R Nature 2000b 405658

Weber Th, Khayyat Kh, Dörner R, Mergel V, Jagutzki O, Schmidt L, Afaneh F, Gonzalez A, Cocke C L, Lander A L and Schmidt-Böcking H 2000c J. Phys. B 333331

Weber Th, Khayyat Kh, Dörner R, Rodríguez V D, Mergel V, Jagutzki O, Schmidt L, Müller K A, Afaneh A, Gonzalez A and Schmidt-Böcking H 2001 Phys. Rev. Lett. 86224

Weber Th, Jagutzki O, Hattass M, Staudte A, Nauert A, Schmidt L, Prior M H, Landers A L, Bräuning-Demian A, Bräuning H, Cocke C L, Osipov T, Ali I, Diez Muino R, Rolles D, Garcia de Abajo F J, Fadley C S, Van Hove M A, Cassimi A, Schmidt-Böcking H and Dörner R 2001a J. Phys. B 343669

Weber Th, Weckenbrock M, Staudte A, Hattass M, Spielberger L, Jagutzki O, Mergel V, Schmidt-Böcking H, Urbasch G, Giessen H, Bräuning H P, Cocke C L, Prior M H, Dörner R 2001b Optics Express 8368

Weber Th, Weckenbrock M, Balser M, Schmidt L, Jagutzki O, Arnold W, Hohn O, Arenholz E, Young T, Osipov T, Foucar L, De Fanis A, Diez Muino R, Schmidt-Böcking H, Cocke C L, Prior M H and Dörner R 2003 Phys. Rev. Lett. 90153003

Weber Th 2003 PhD-thesis, Johann-Wolfgang-Goethe Universität Frankfurt

Weckenbrock M, Hattass M, Czasch A, Jagutzki O, Schmidt 1, Weber T, Roskos H, Löffler H, Thomson M and Dörner R 2001 J. Phys. B 34 L449

Weckenbrock M, Becker A, Staudte A, Kammer S, Smolarski M, Bhardwaj V R, Rayner D, Villeneuve D M, Corkum P B, Dörner R 2003 Phys. Rev. Lett. (submitted)

Whelan C T, Allan R J, Walters H R J and Zhang X 1993 in $(e, 2 e)$ \& related processes edited by Whelan $\mathrm{C} T$ et al, Kluwer, Dordrecht 1-32

Winter T G, Lin C D 1984 Phys. Rev. A 293071

Wolf S and Helm H 2000 Phys. Rev. A 62043408

Wolff W, Ben-Itzhak I, Wolf H E, Cocke C L, Abdallah M A and Stöckli M 2002 Phys. Rev. A 65042710

Wollenhaupt M, Assion A, Liese D, Sarpe-Tudoran Ch, Baumert T, Zamith S, Bouchene M A, Girard B, Flettner A, Weichmann U and Gerber G 2002 Phys. Rev. Lett. 89 1730011

Wood C J, Olson R E, Schmitt W, Moshammer R and Ullrich J 1997 Phys. Rev. A 563746

Wood C J and Olson R E 1999 Phys. Rev. A 591317 
Wu W, Wong K L, Ali R, Chen C Y, Cocke C L, Frohne V, Giese J P, Raphaelian M, Walch B, Dörner R, Mergel V, Schmidt-Böcking H and Meyerhof W E 1994 Phys. Rev. Lett. 723170

Wu W, Wong K L, Cocke C L, Giese J P and Montenegro E C 1995 Phys. Rev. A 513718

Yamanouchi K 2002 Science 2951659

Zähringer K, Meyer H D and Cerderbaum L S 1992 Phys. Rev. A 465643

Zare R N 1972 Molecular Photochem. 41

Zhang H, Fléchard X, Cassimi A, Adoui L, Cremer G, Frémont F and Hennecart D 2001 Phys. Rev. A 64012715

Zouros T J M Comments 1996 At. Mol. Phys. 32, 291 


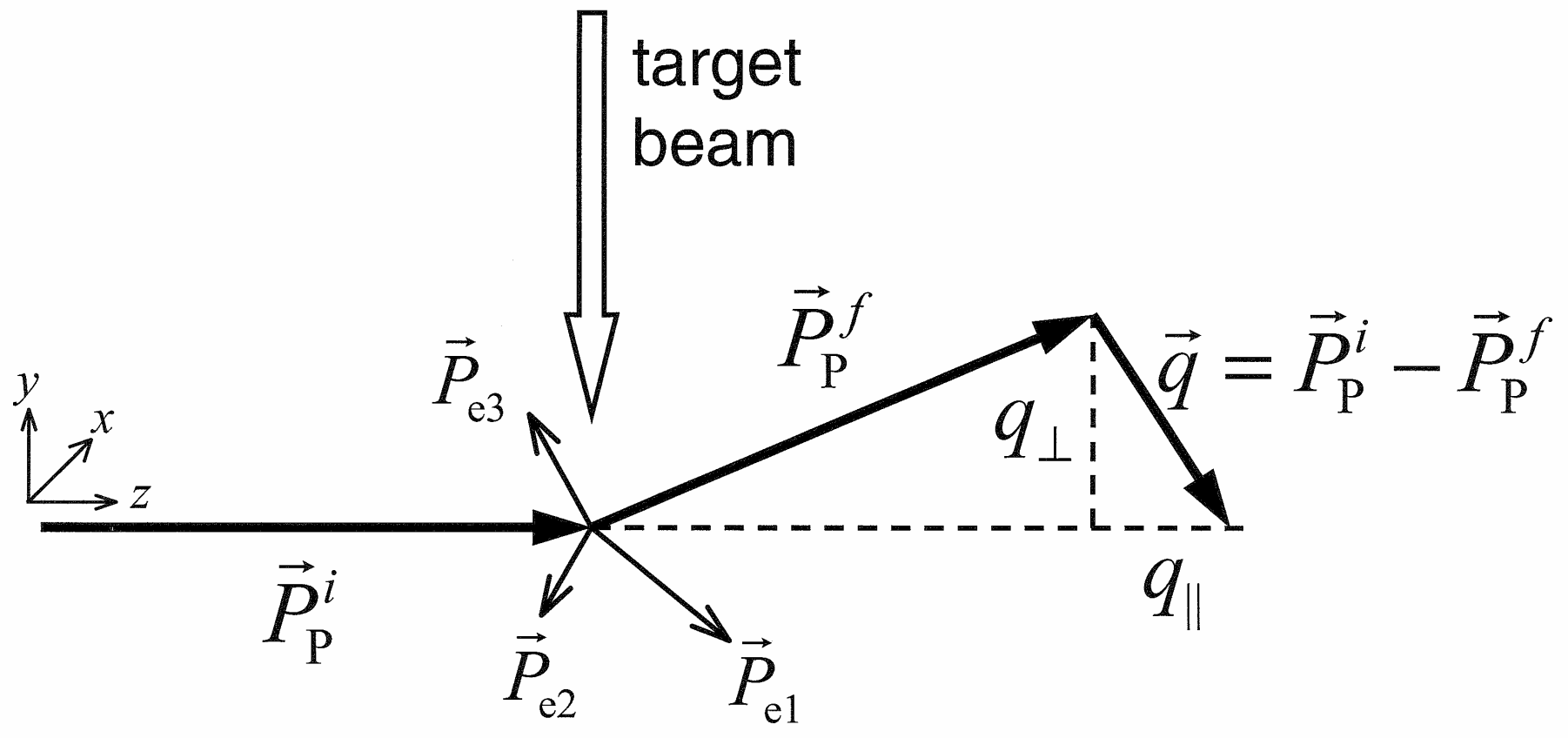

Figure 1 
Electron capture

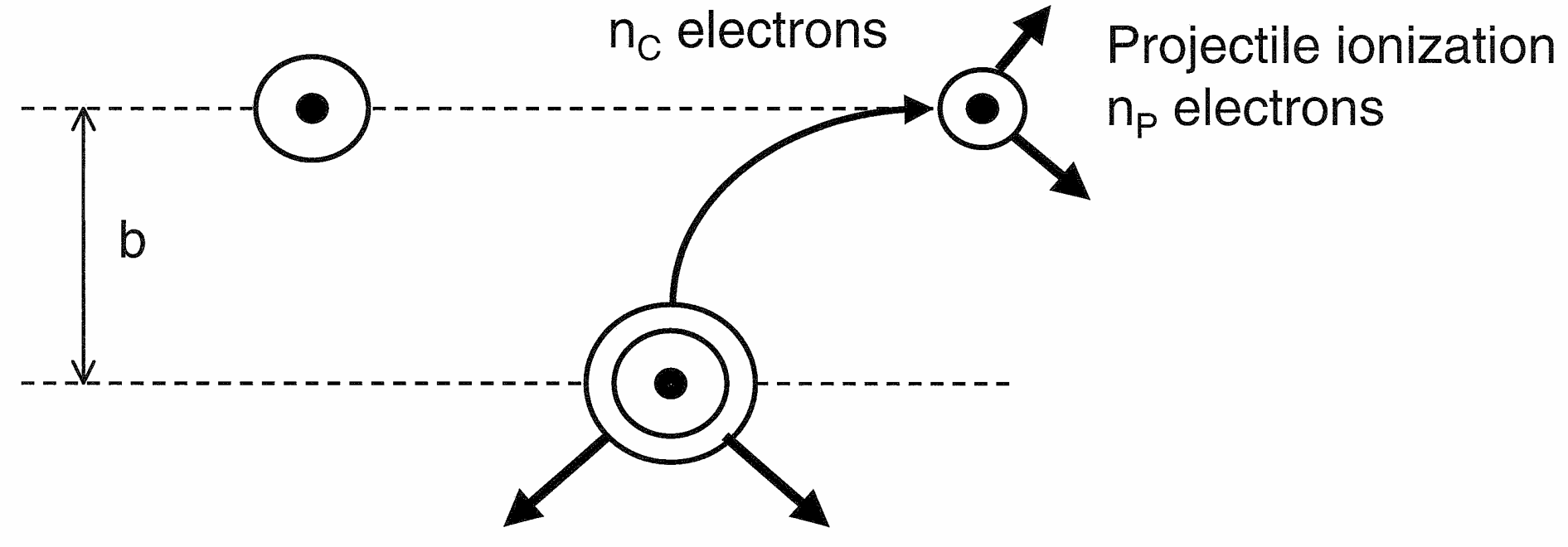

Target ionization

$\mathrm{n}_{\mathrm{T}}$ electrons

Figure 2 


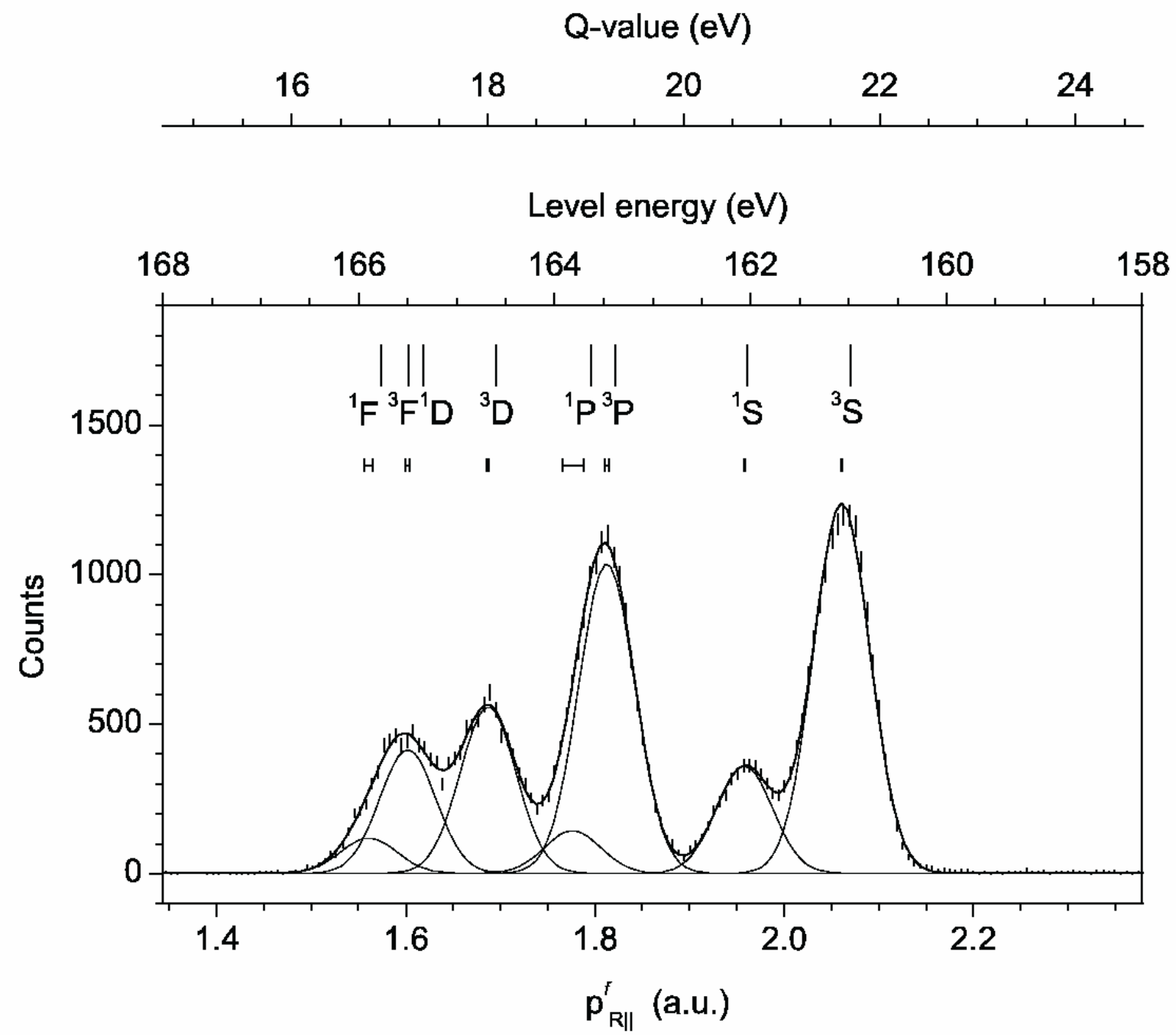

Figure 3 

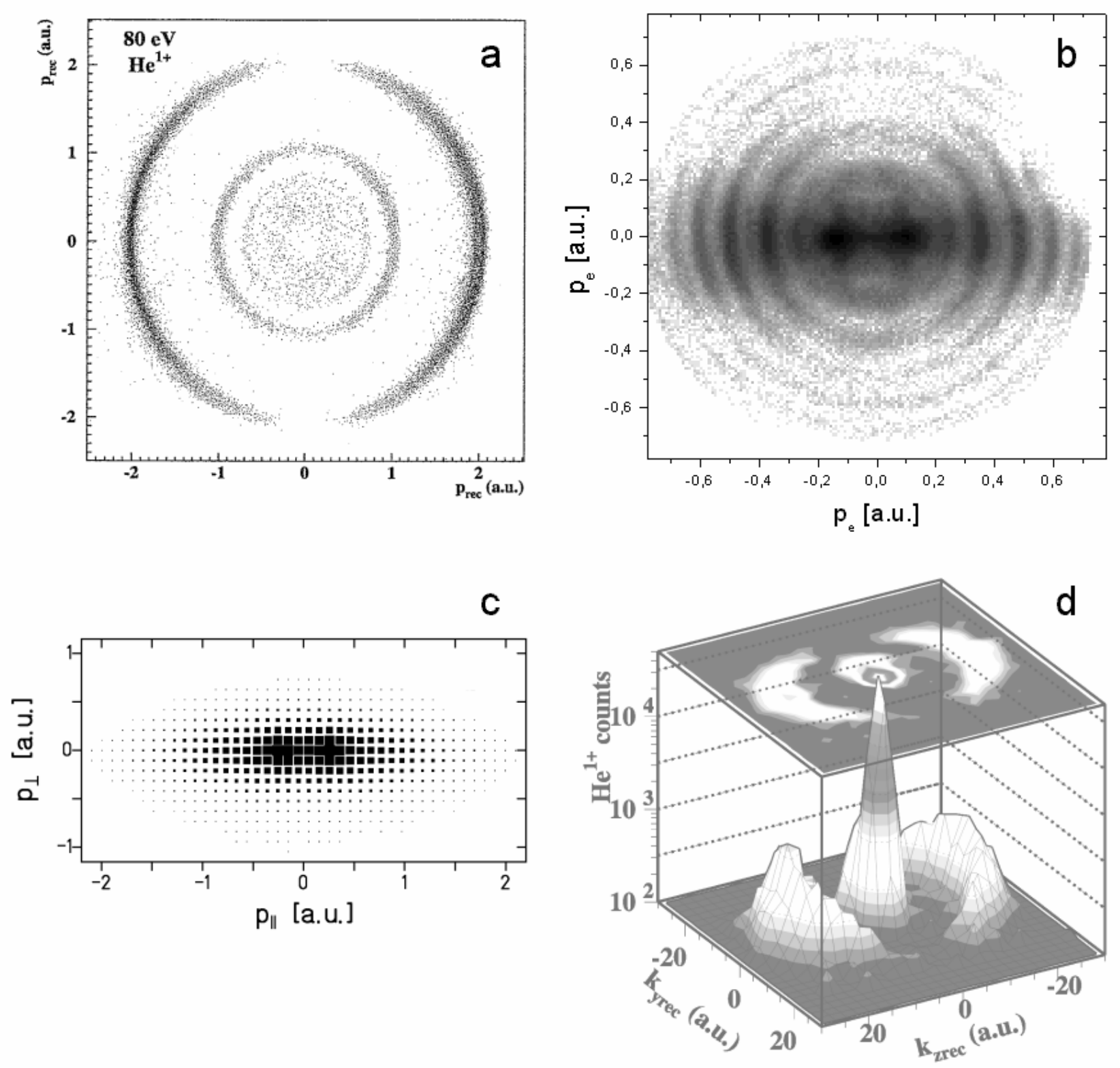

Figure 4 


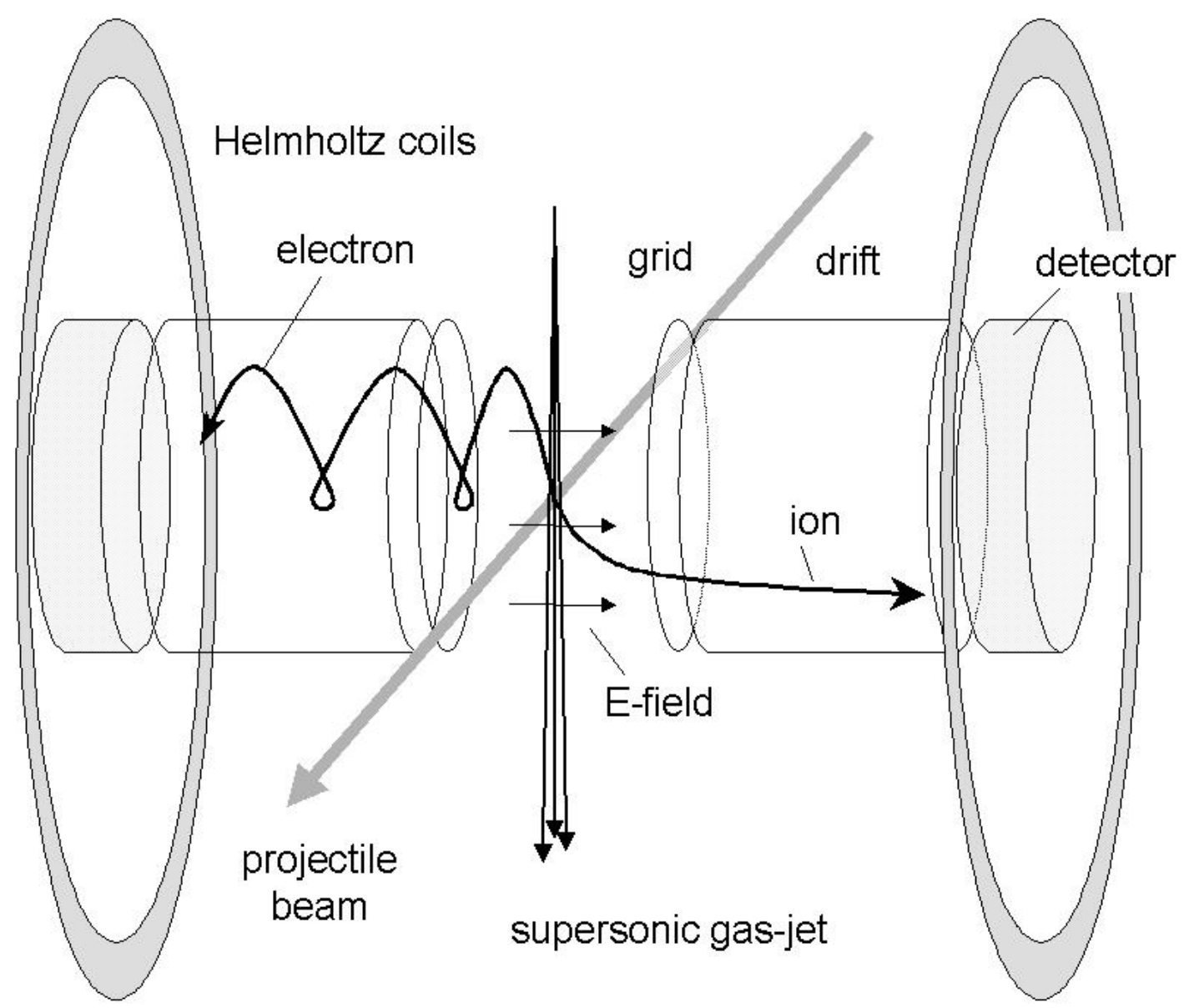

Figure 5 


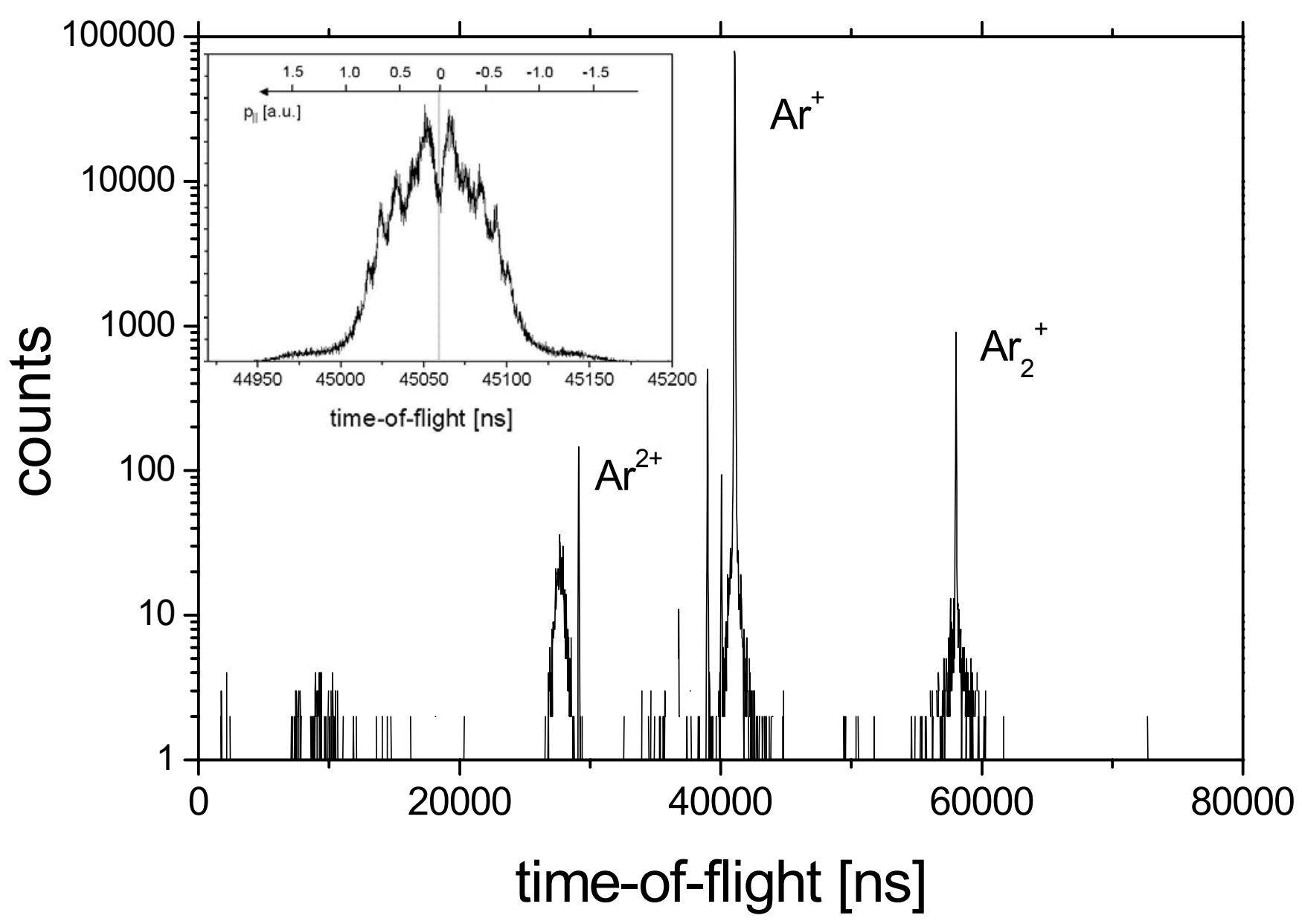

Figure 6 


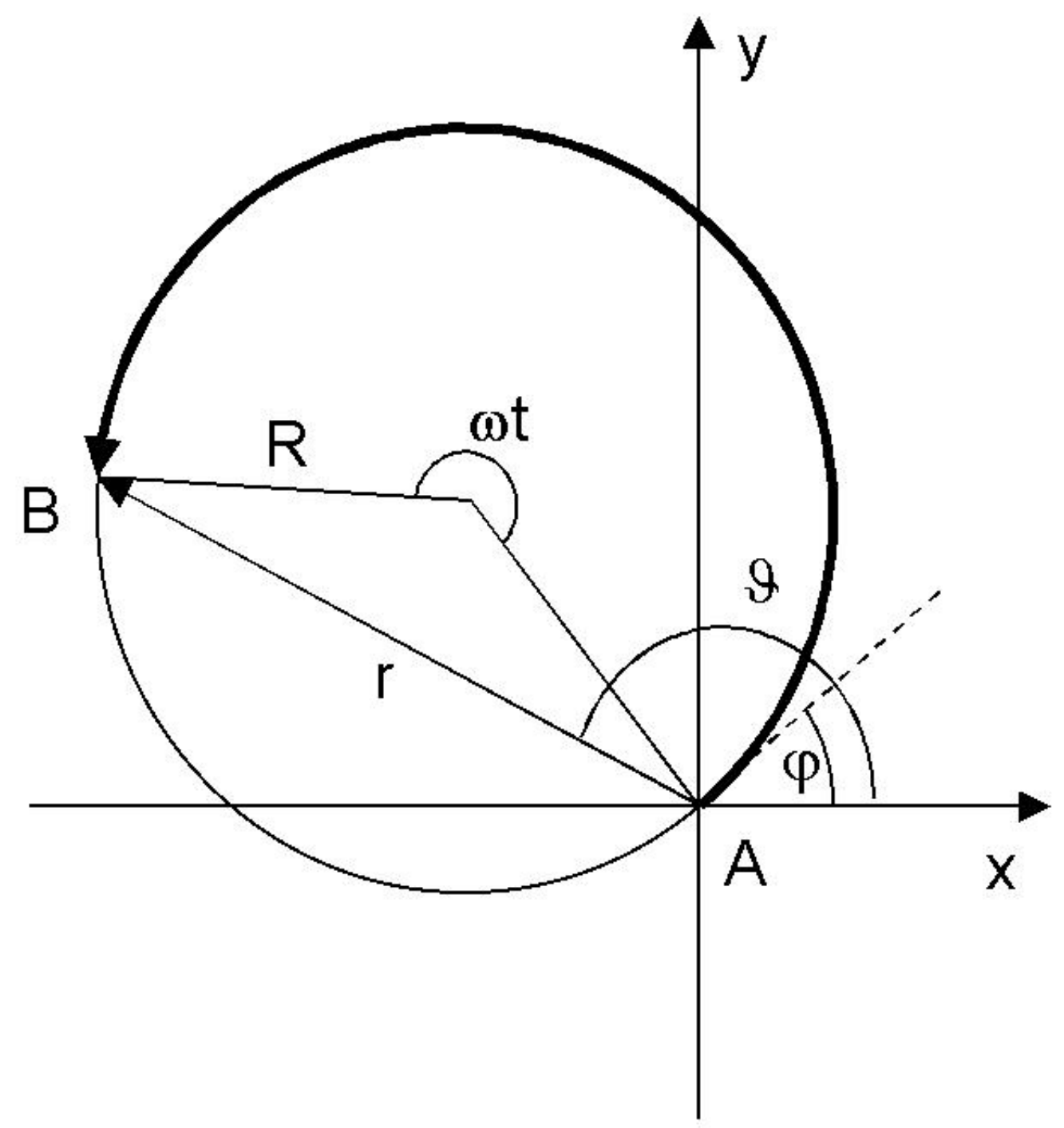

Figure 7 


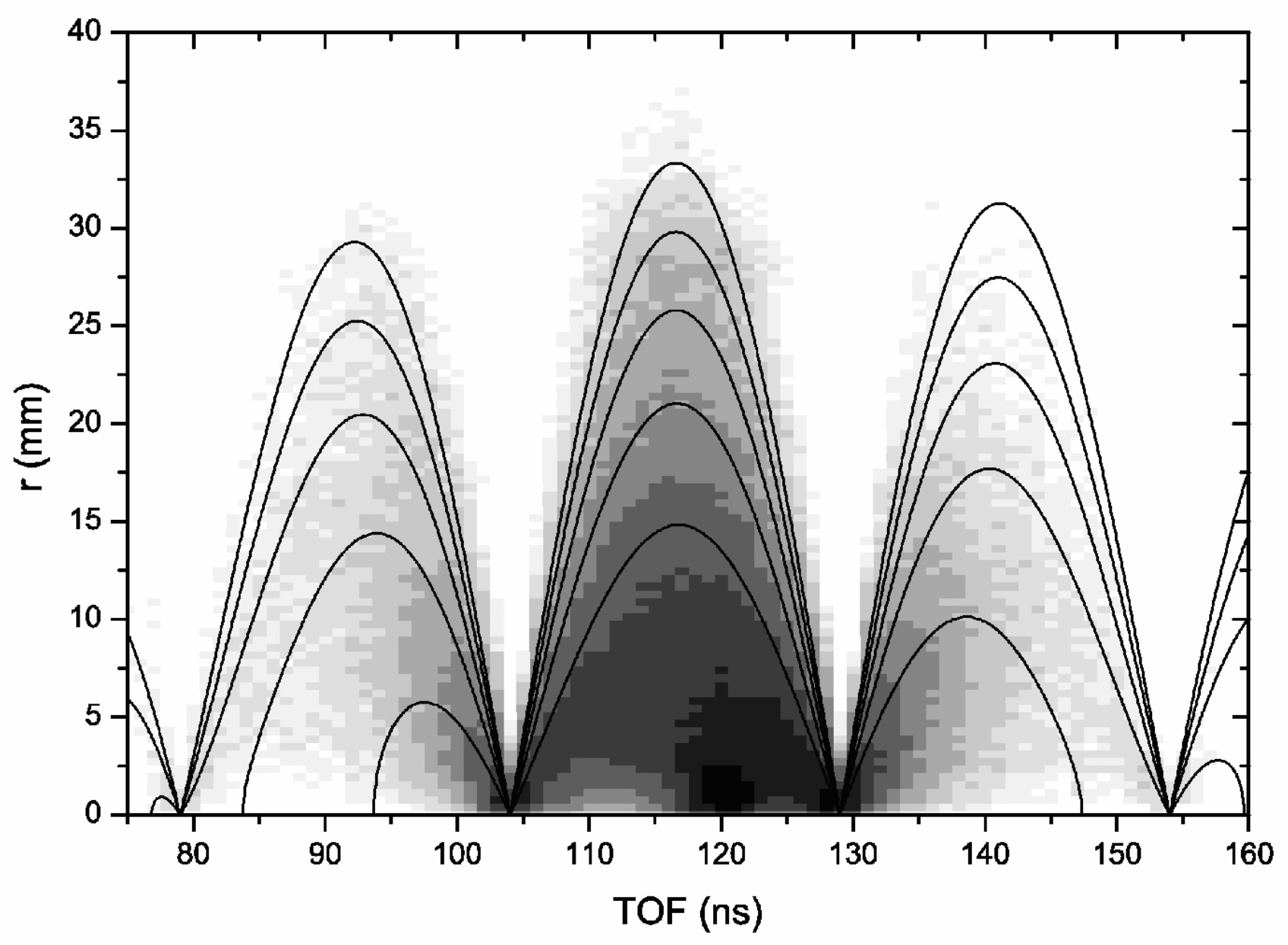

Figure 8 


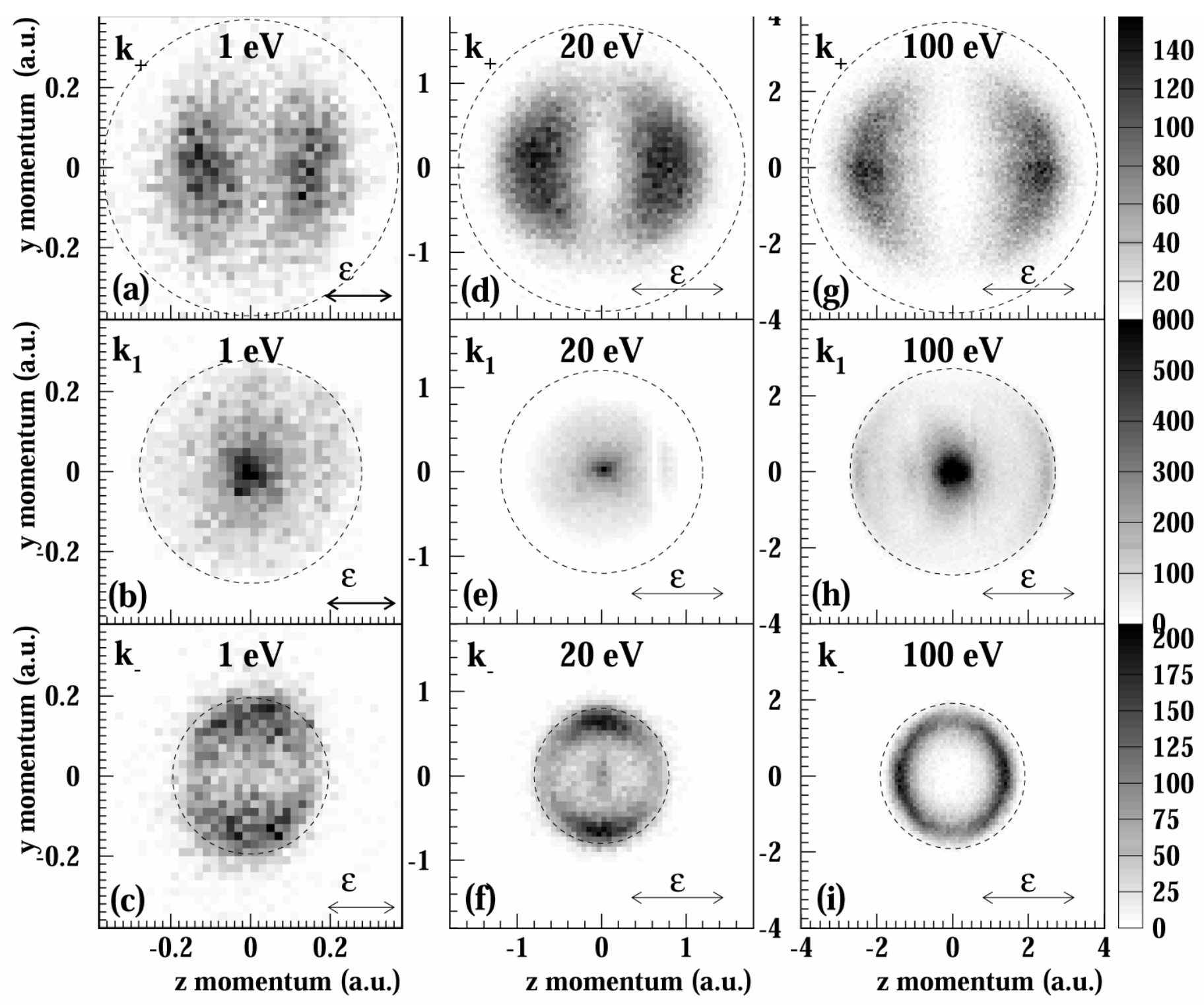

Figure 9 


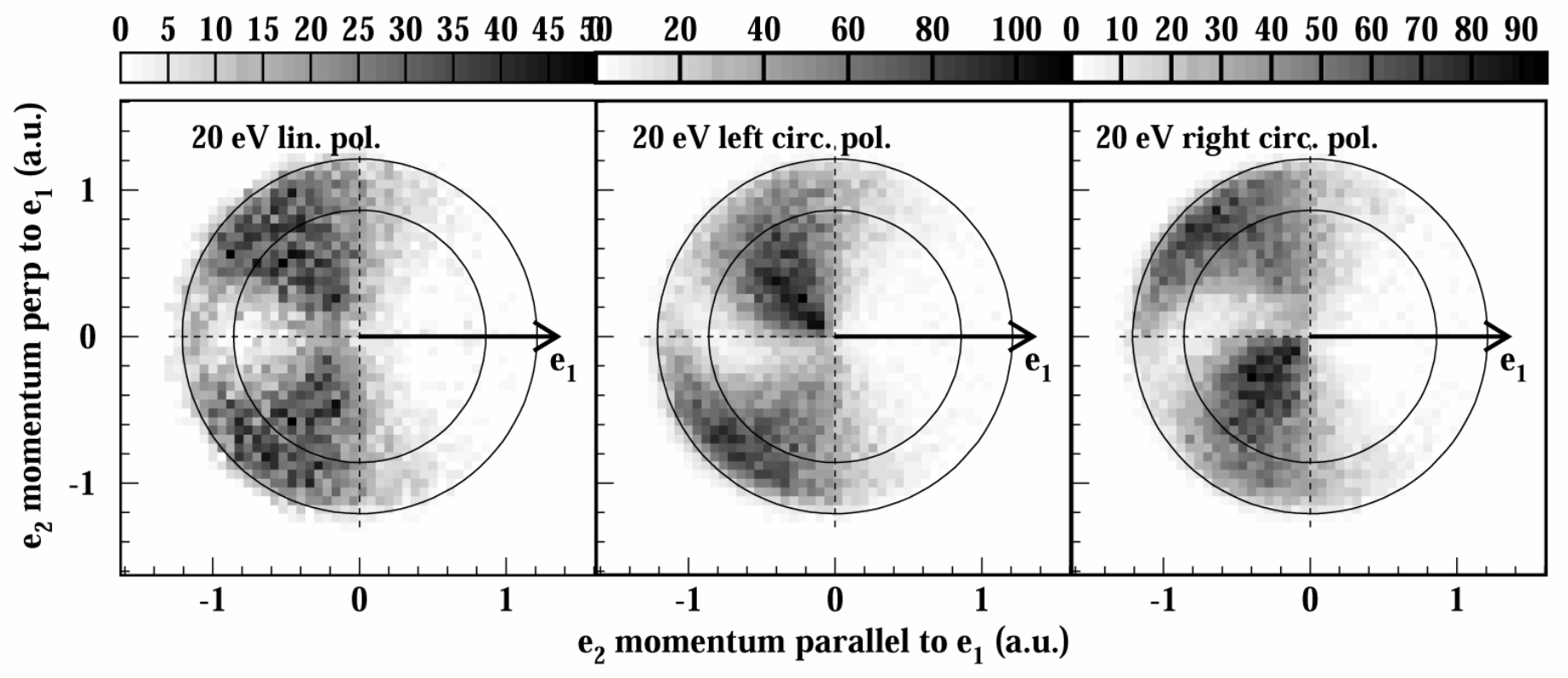

Figure 10 


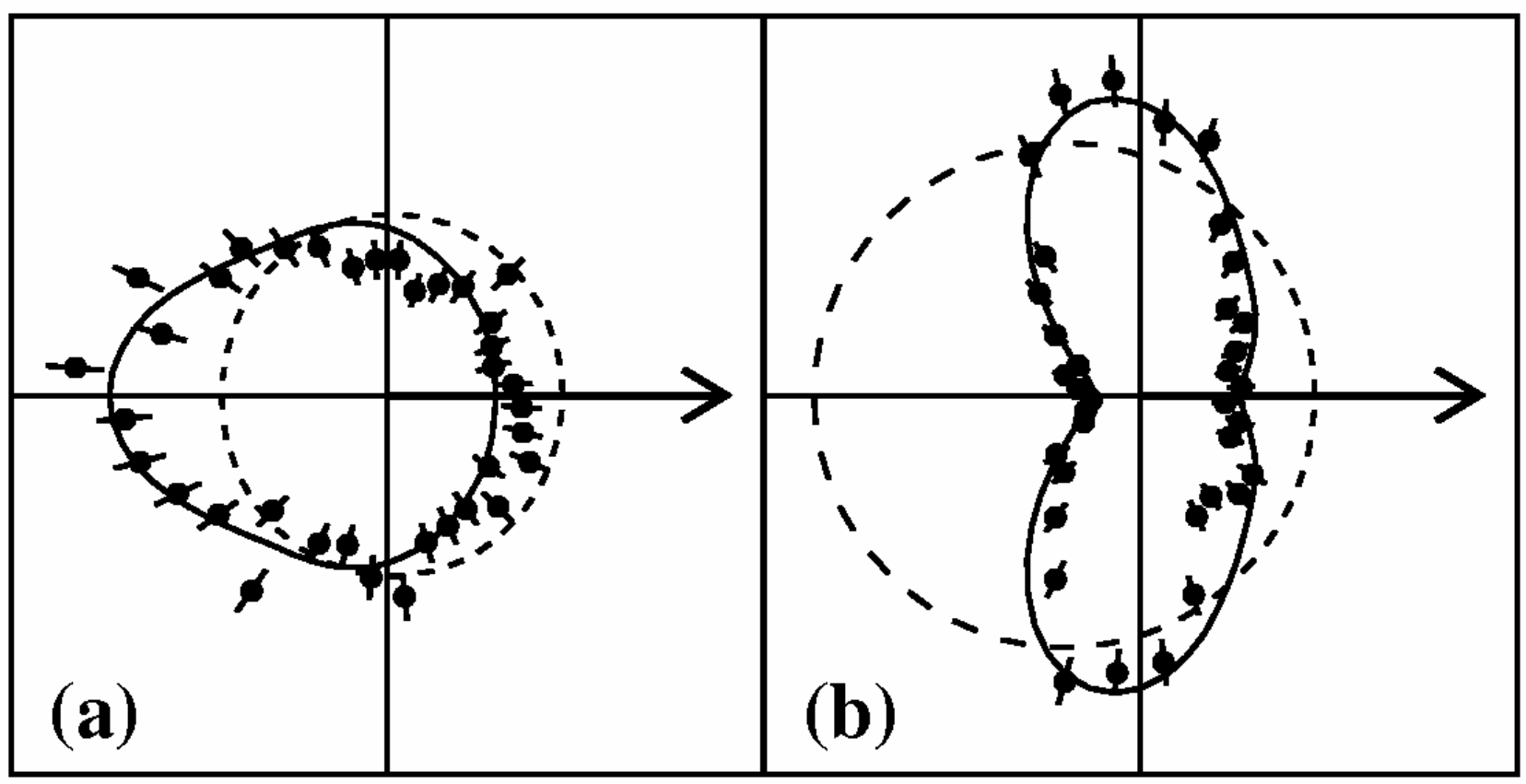

Figure 11 

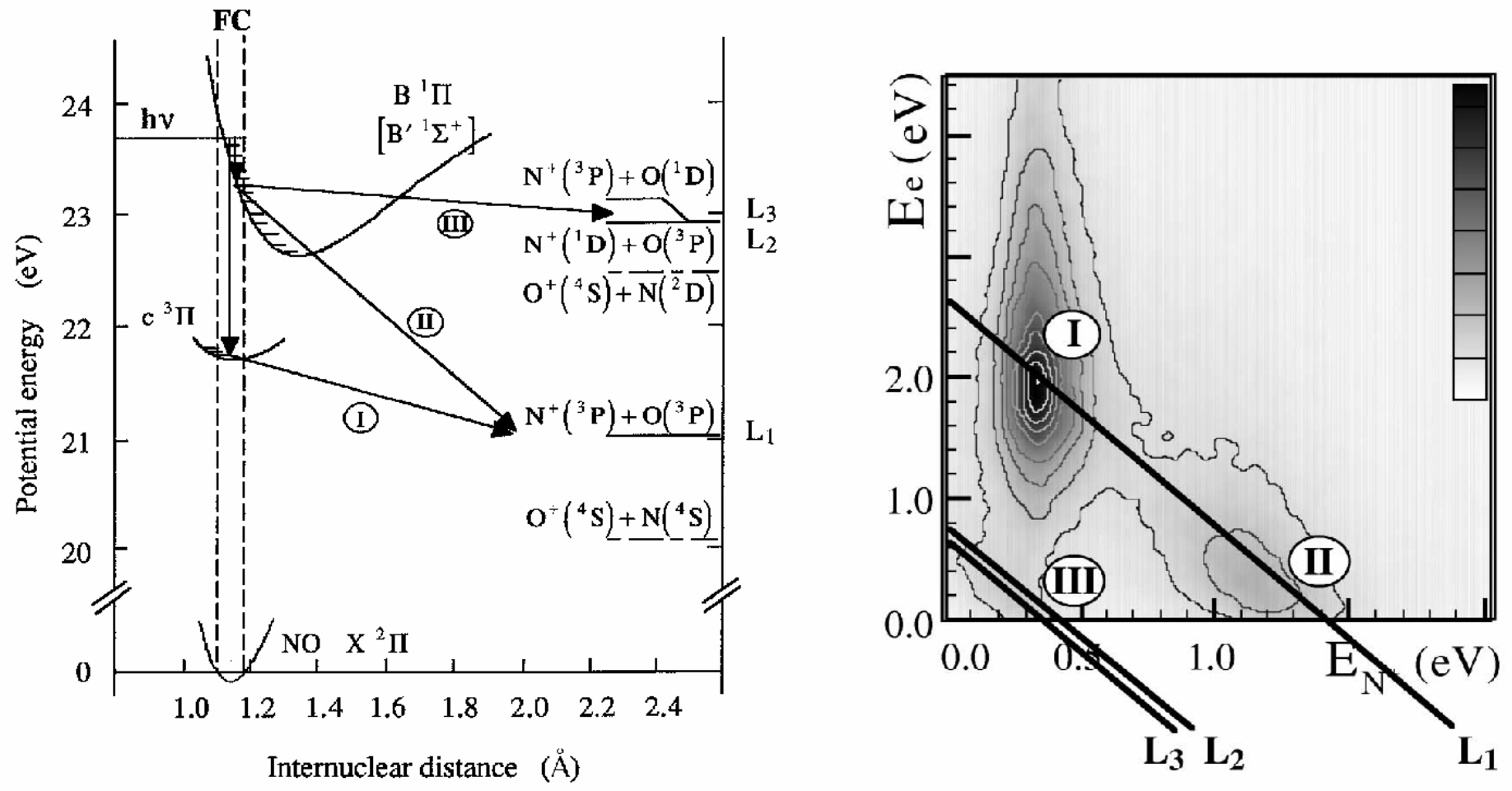

Figure 12 

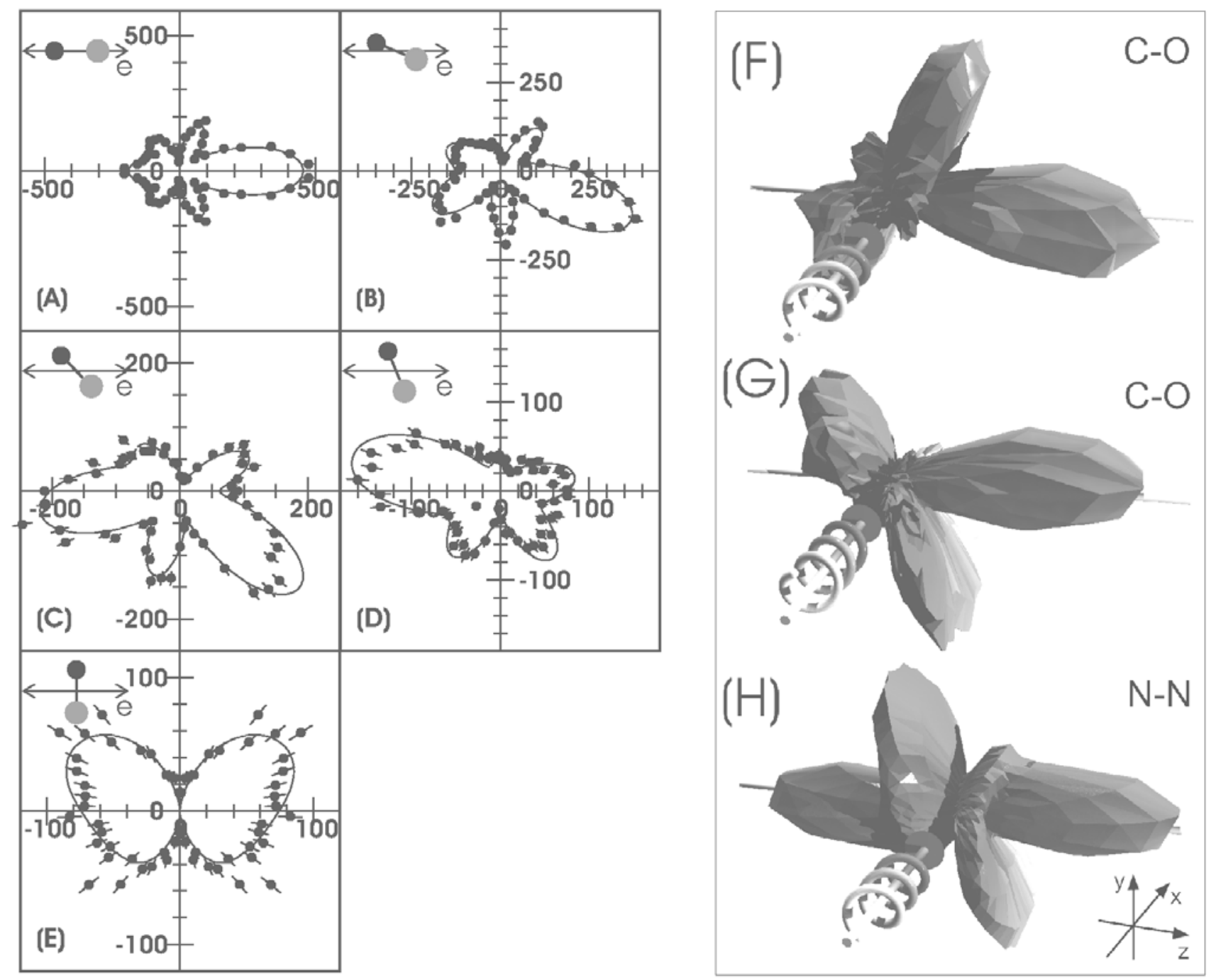

Figure 13 


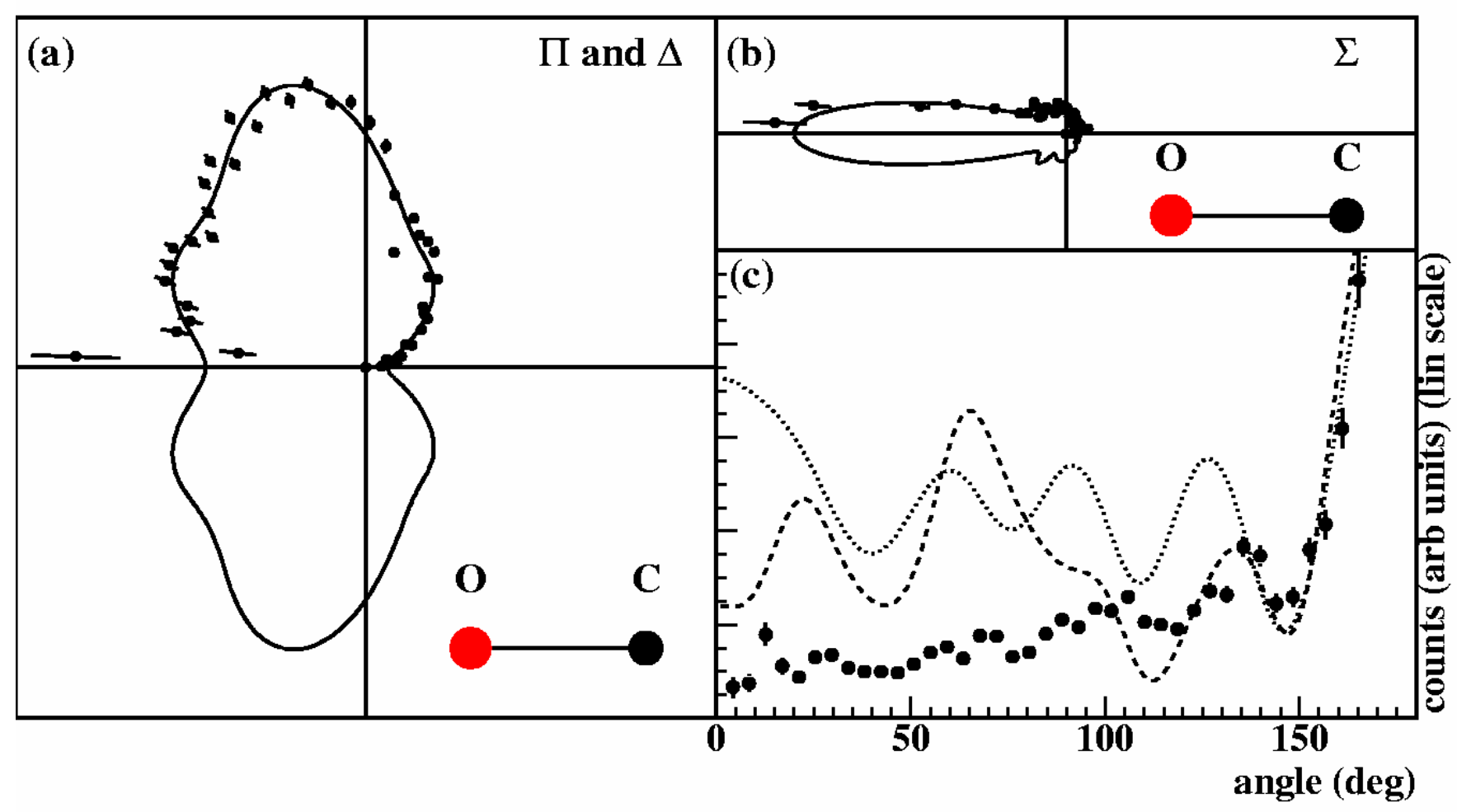

Figure 14 

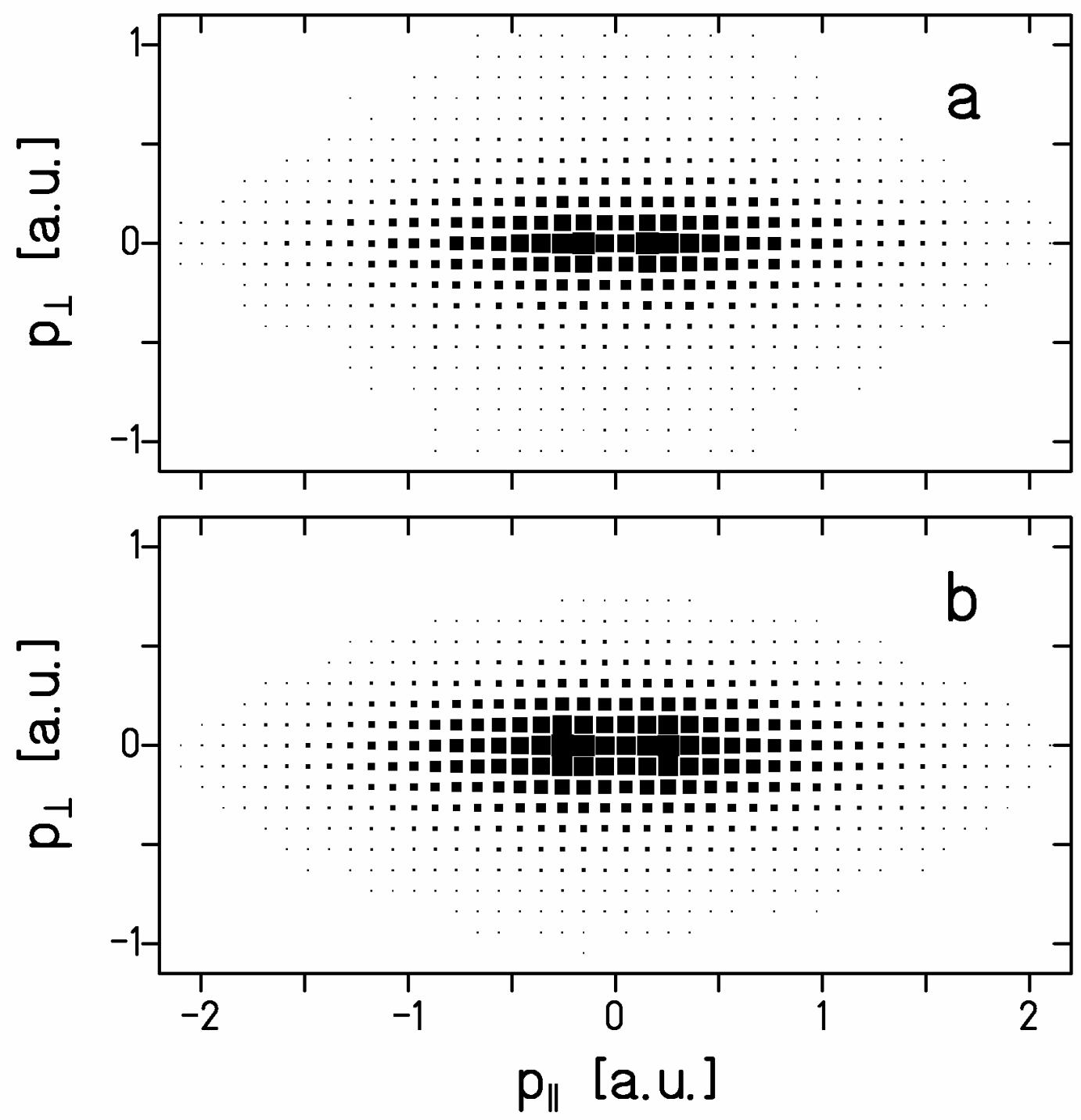

Figure 15 


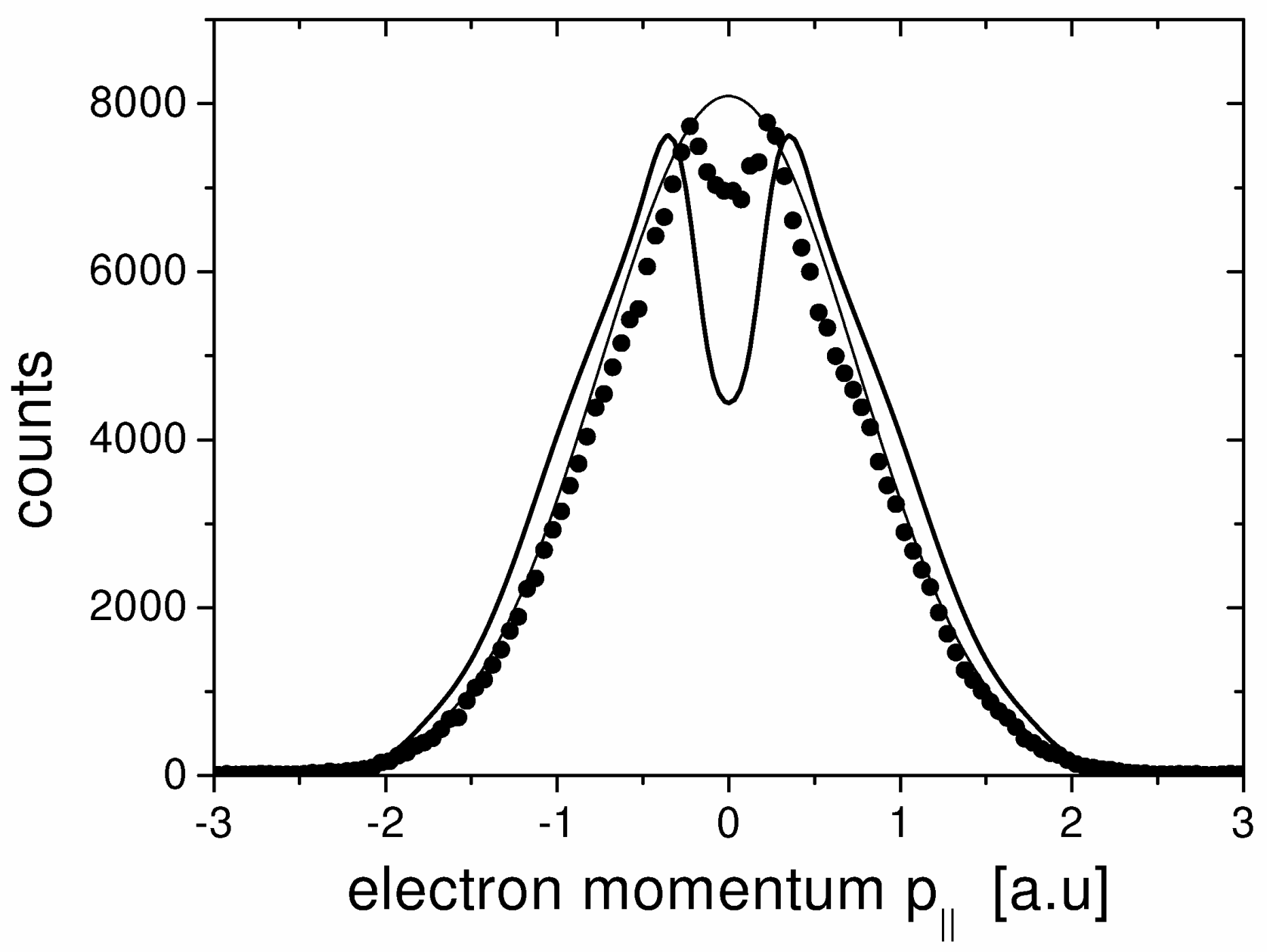

Figure 16 

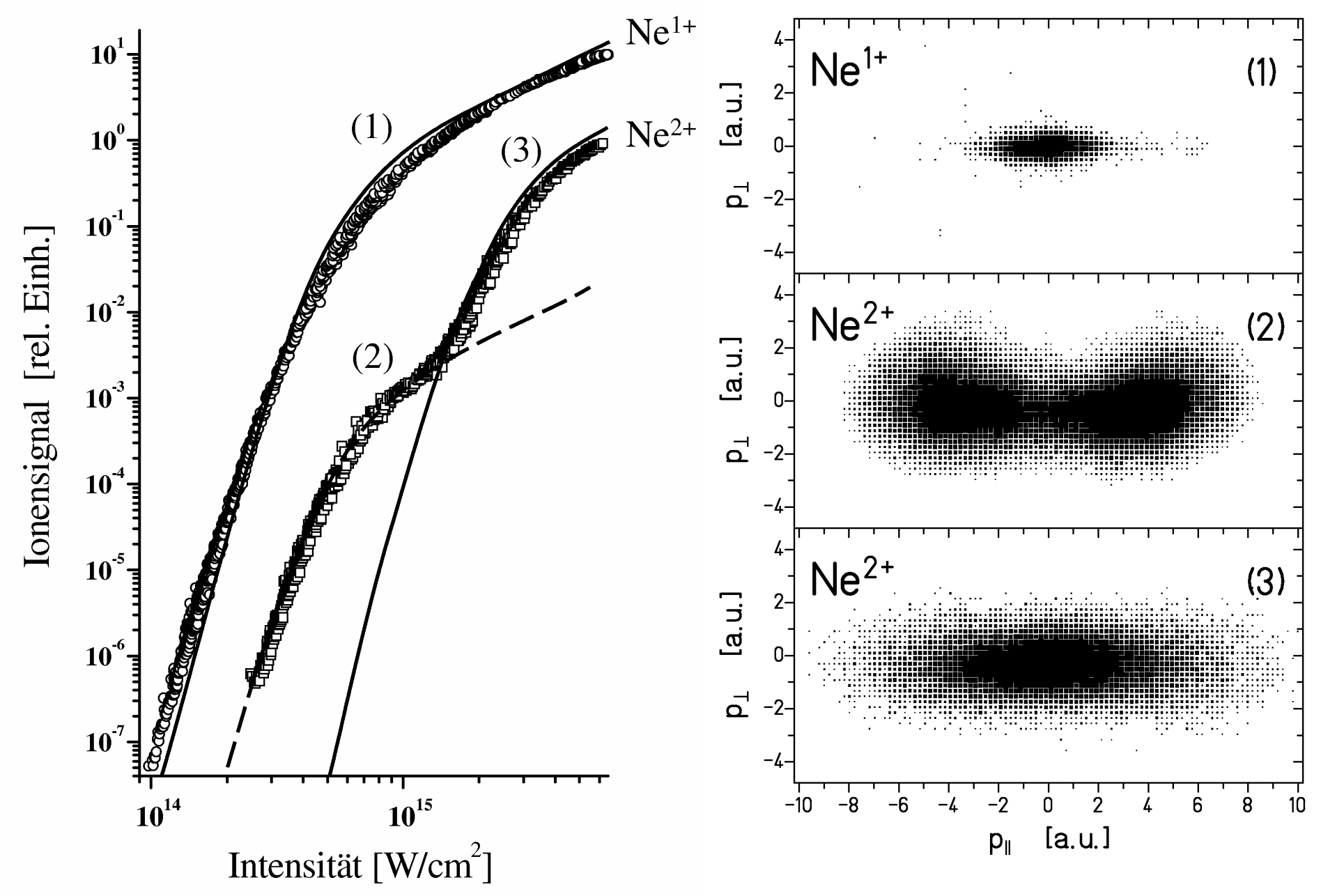

Figure 17 


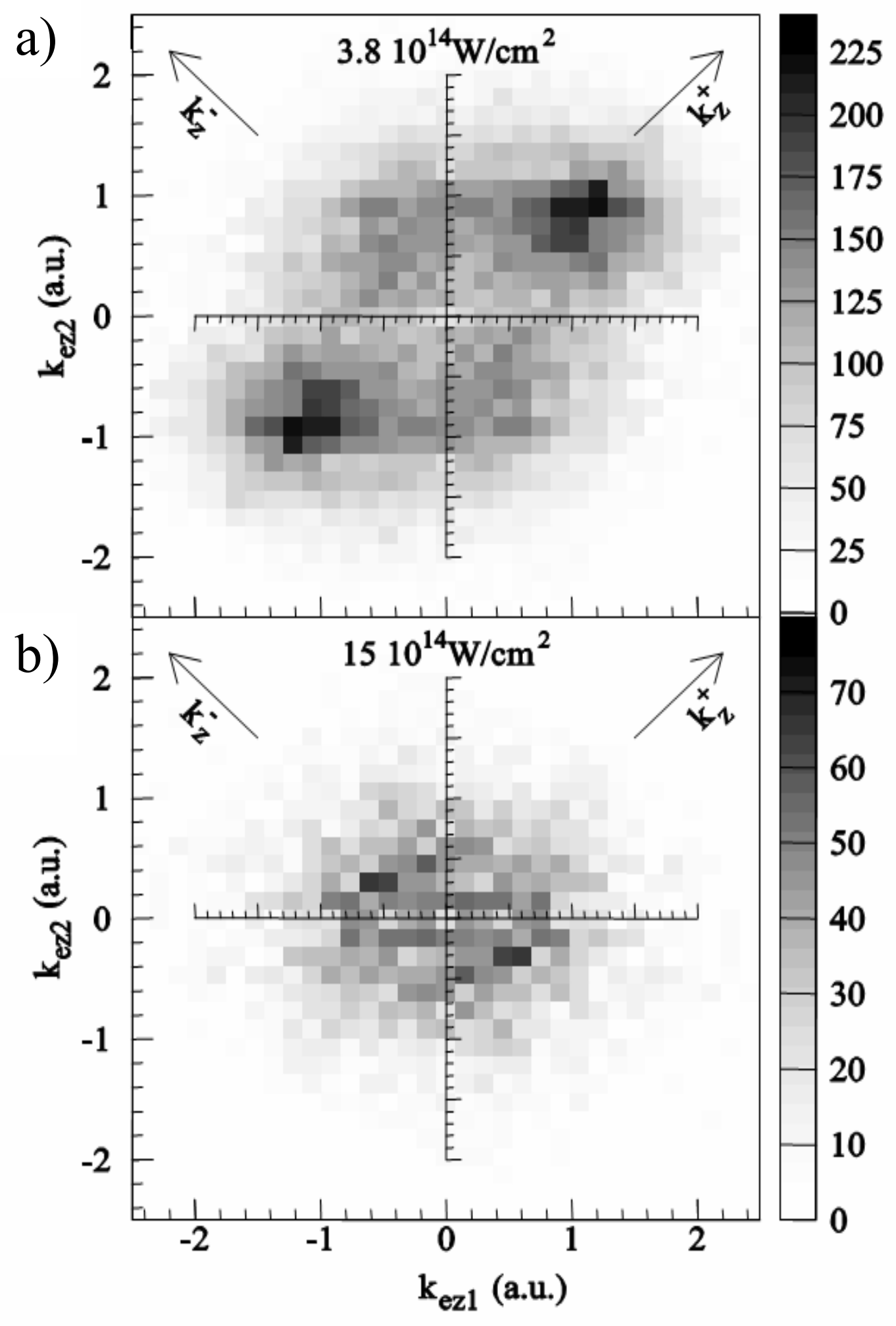

Figure 18 

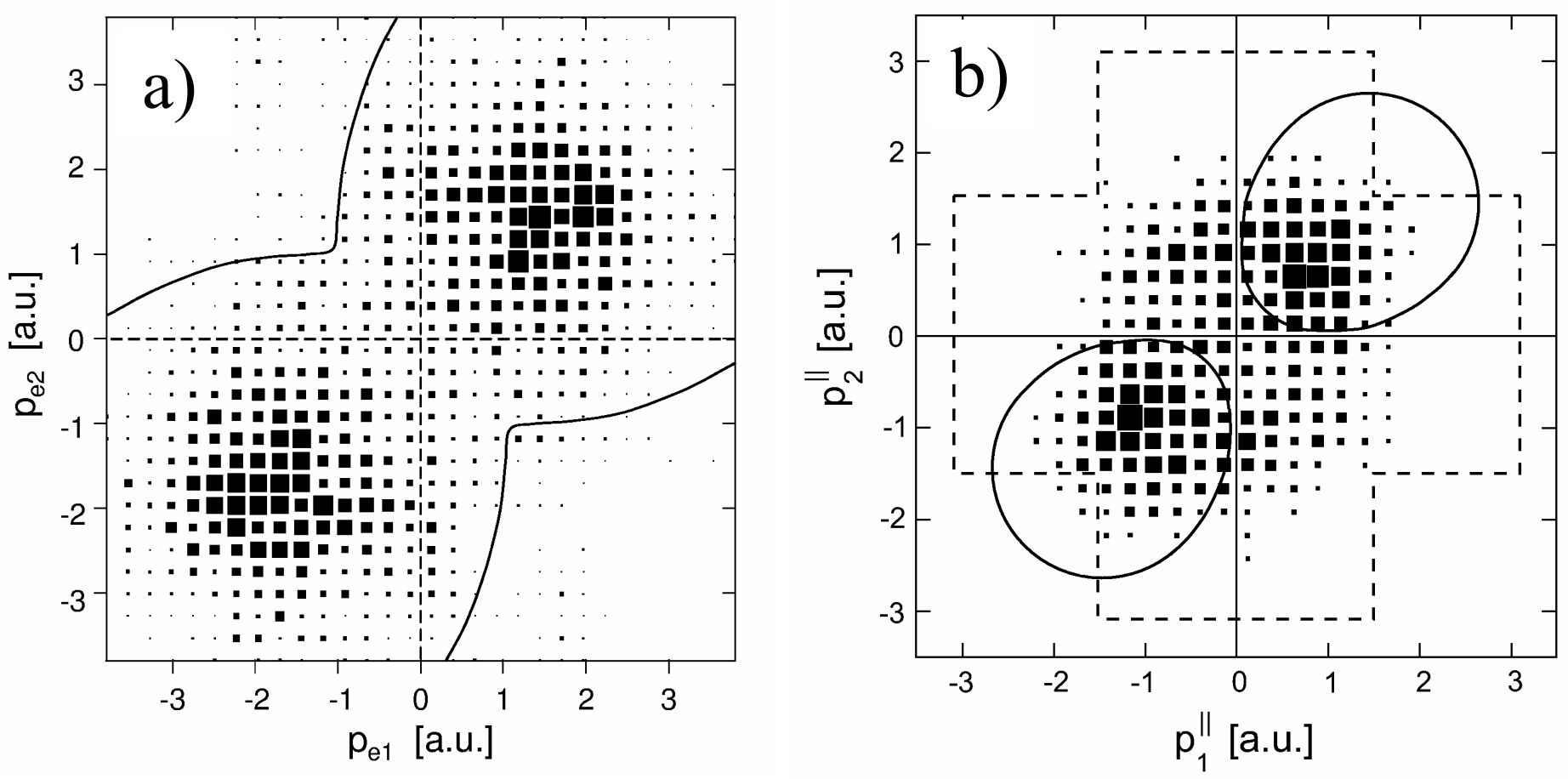

Figure 19 

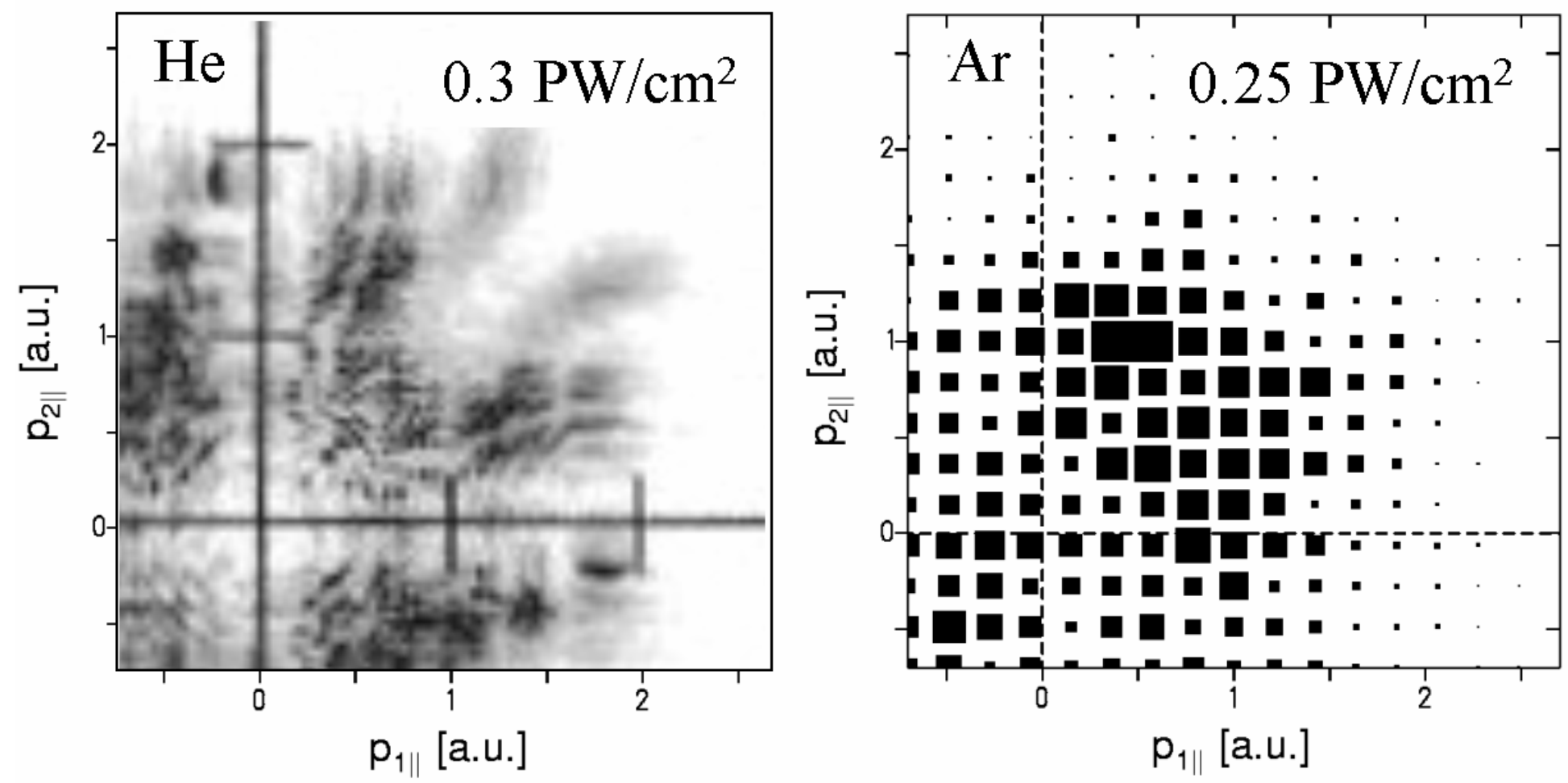

Figure 20 


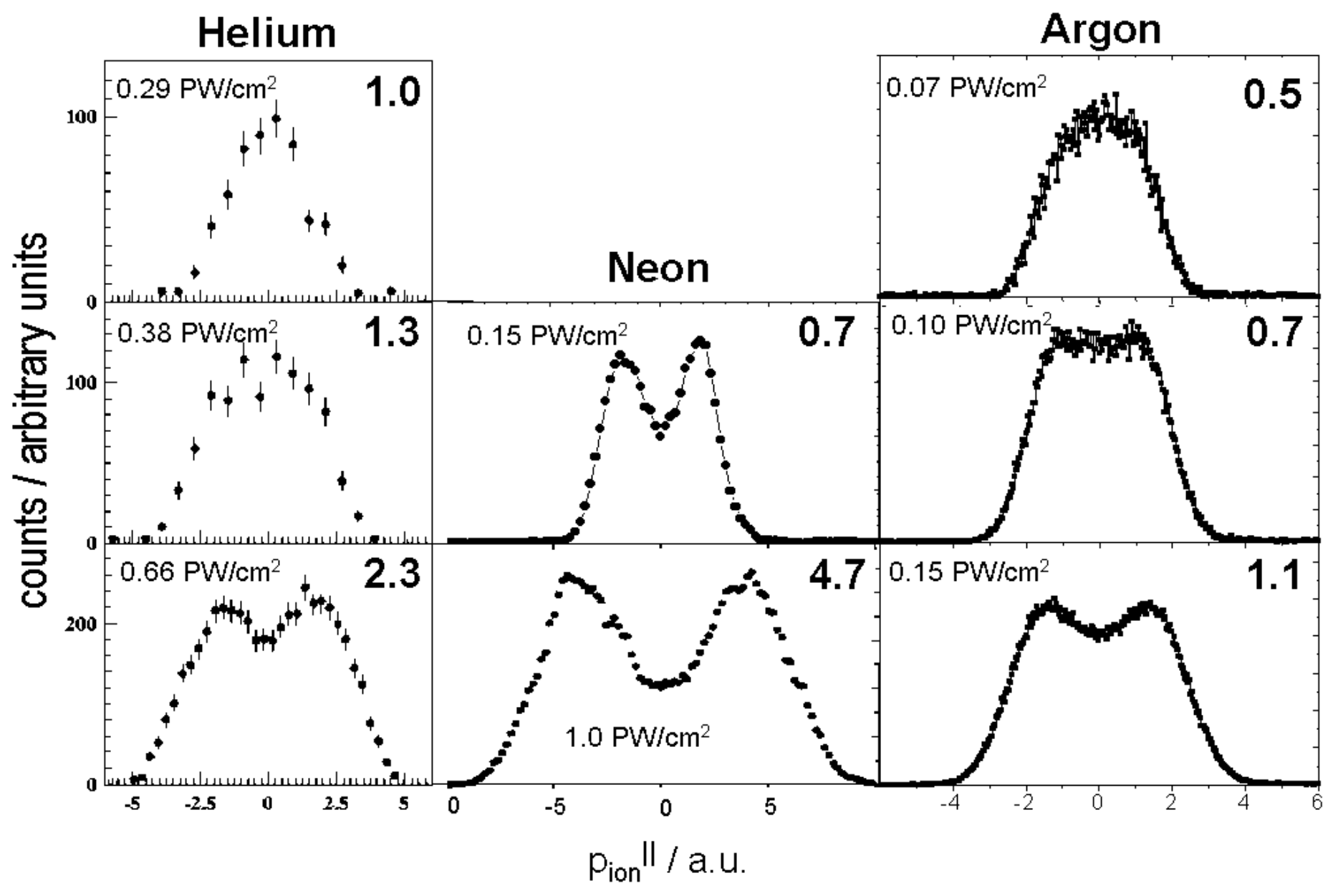

Figure 21 


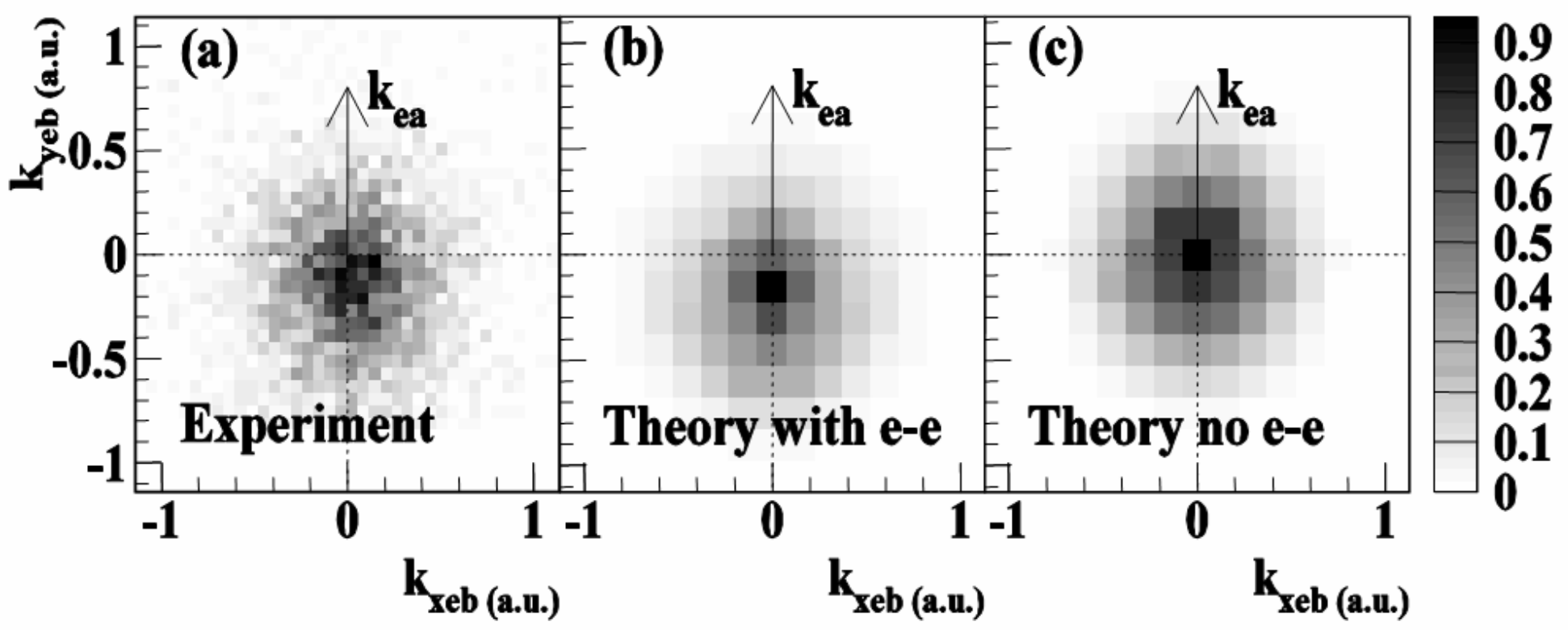

Figure 22 


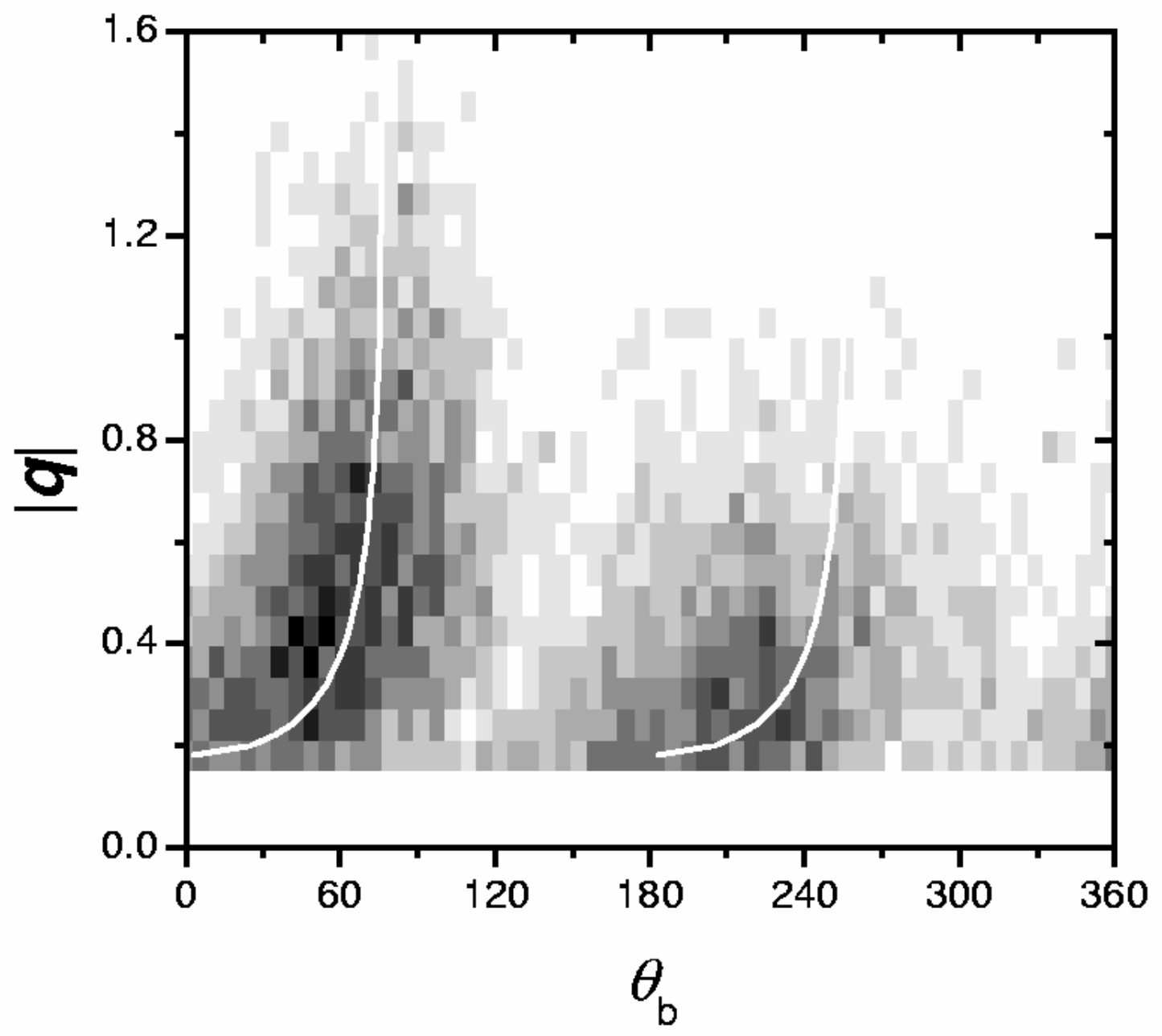

Figure 23 


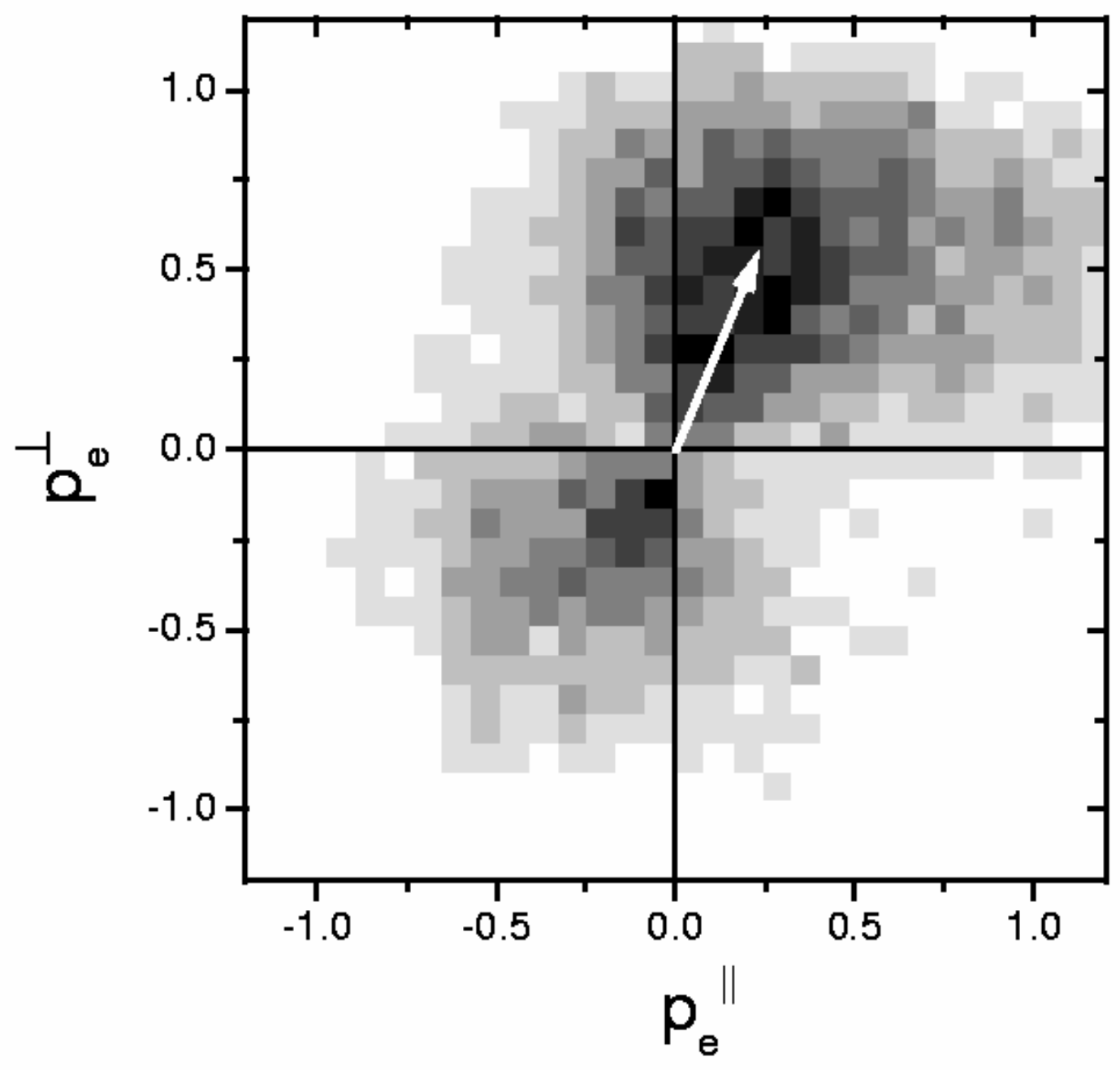

Figure 24 
a)

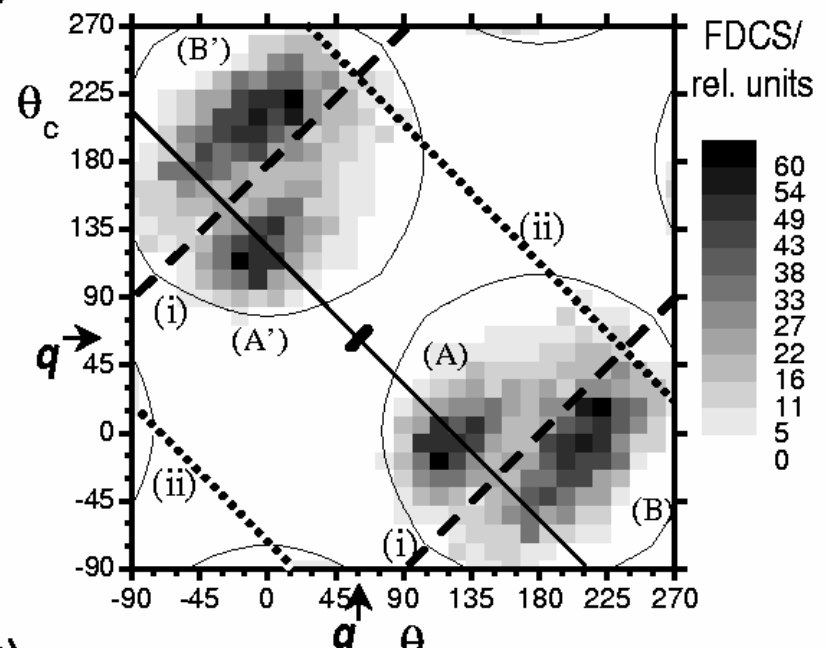

c)

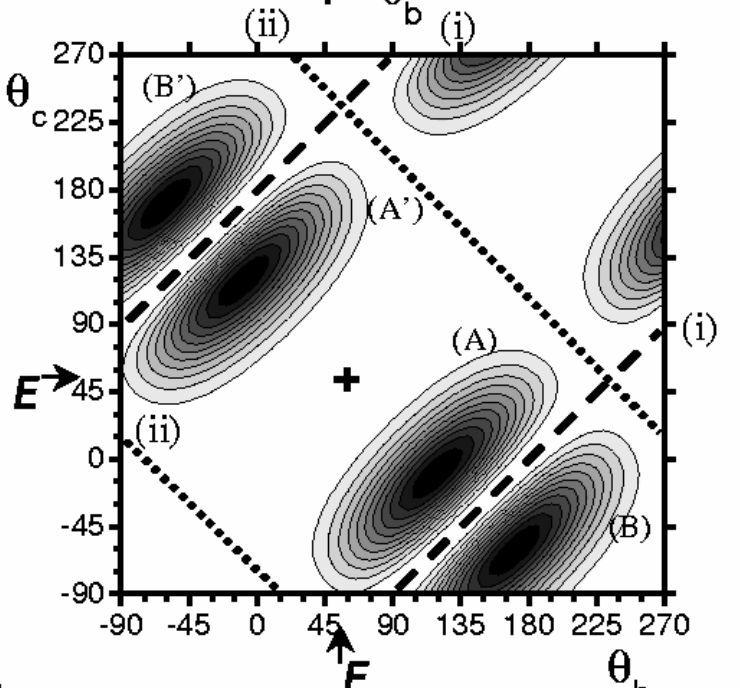

e)

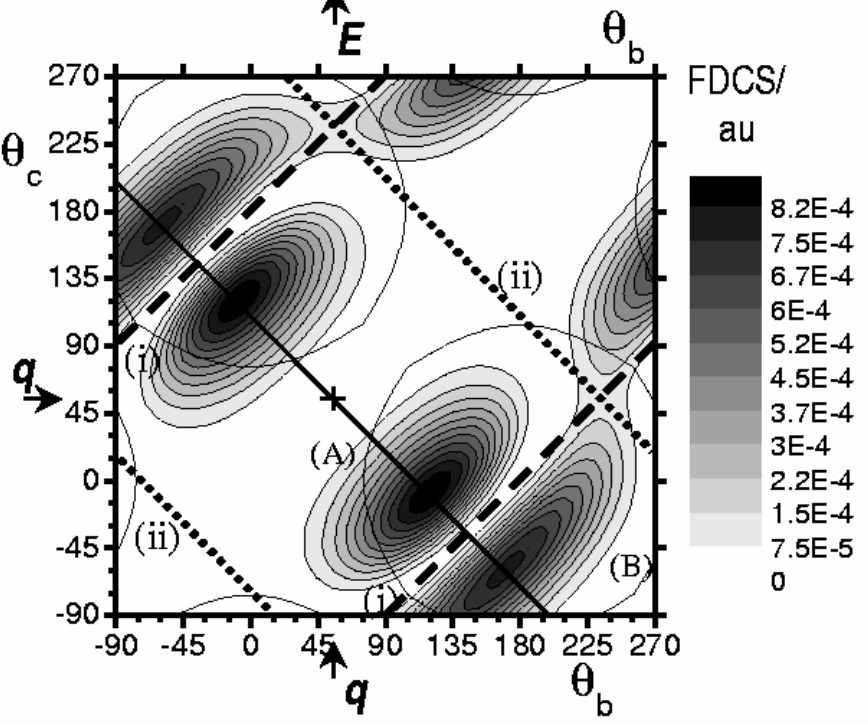

b)

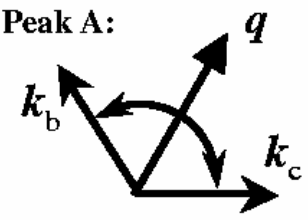

Peak B:

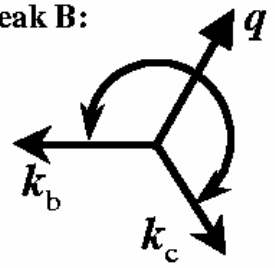

d)

Peak A:

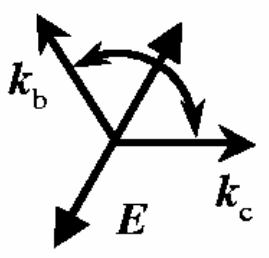

Peak B:

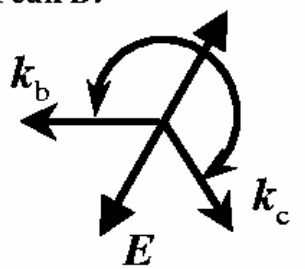

Figure 25 


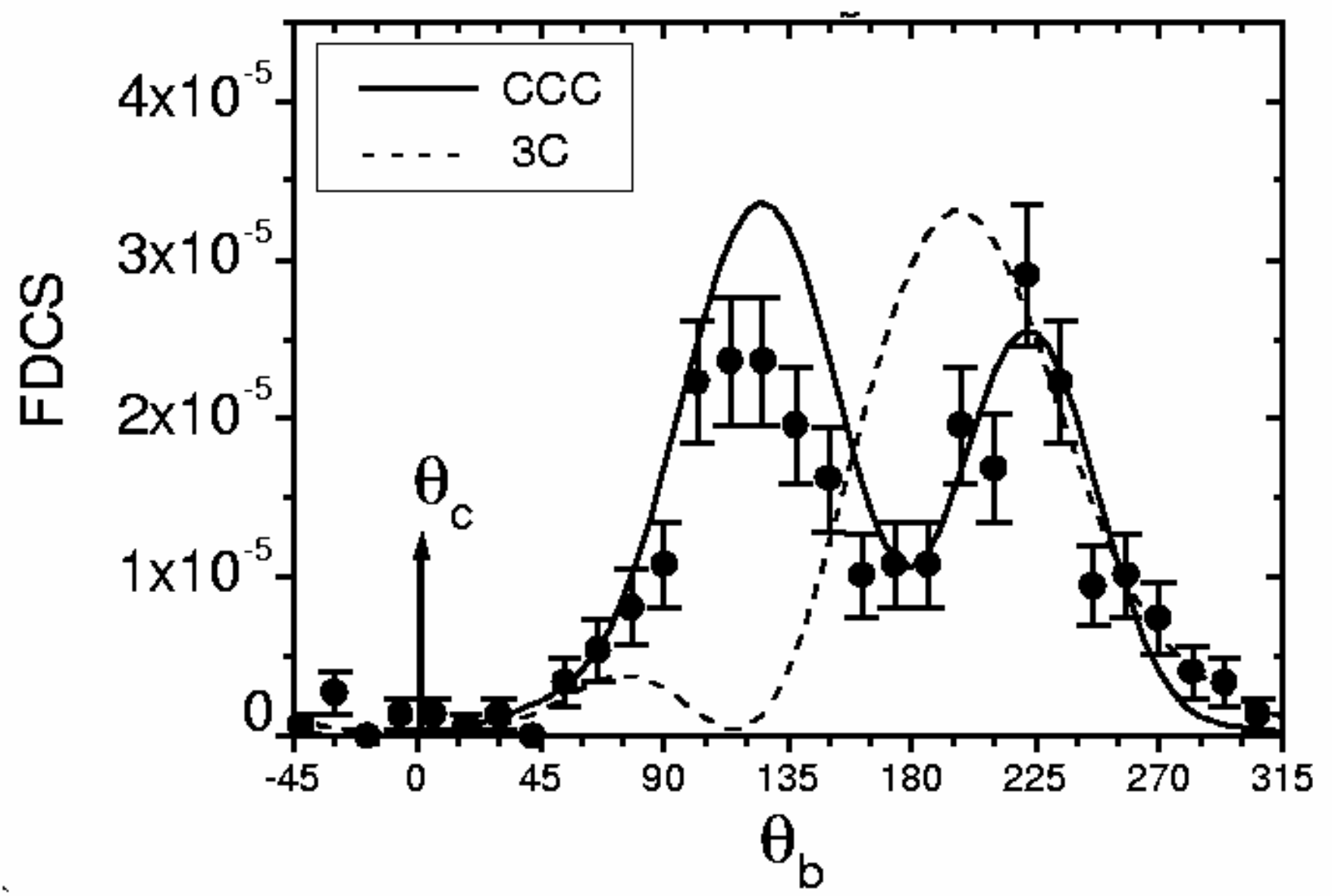

Figure 26 


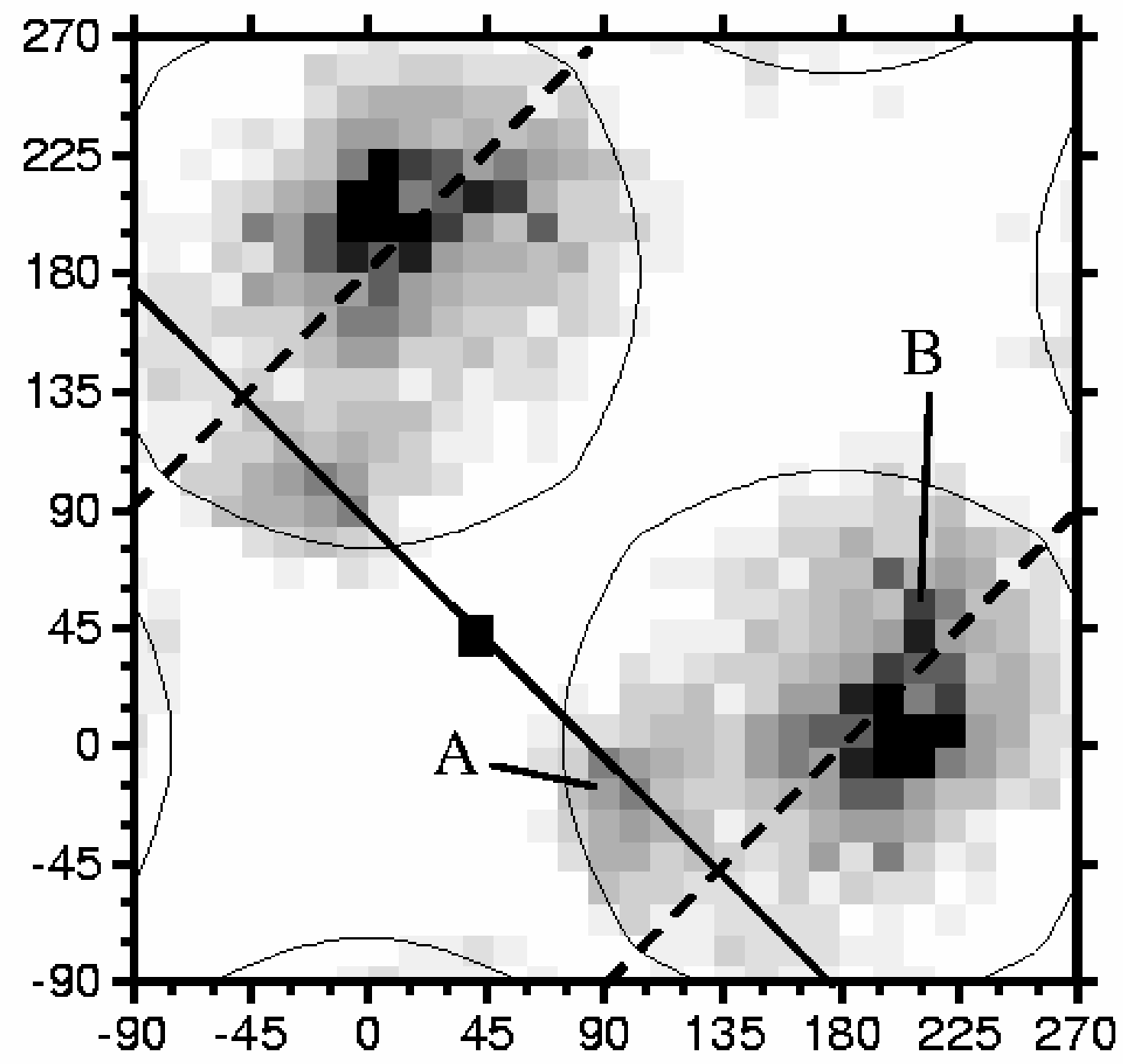

Figure 27 
a)

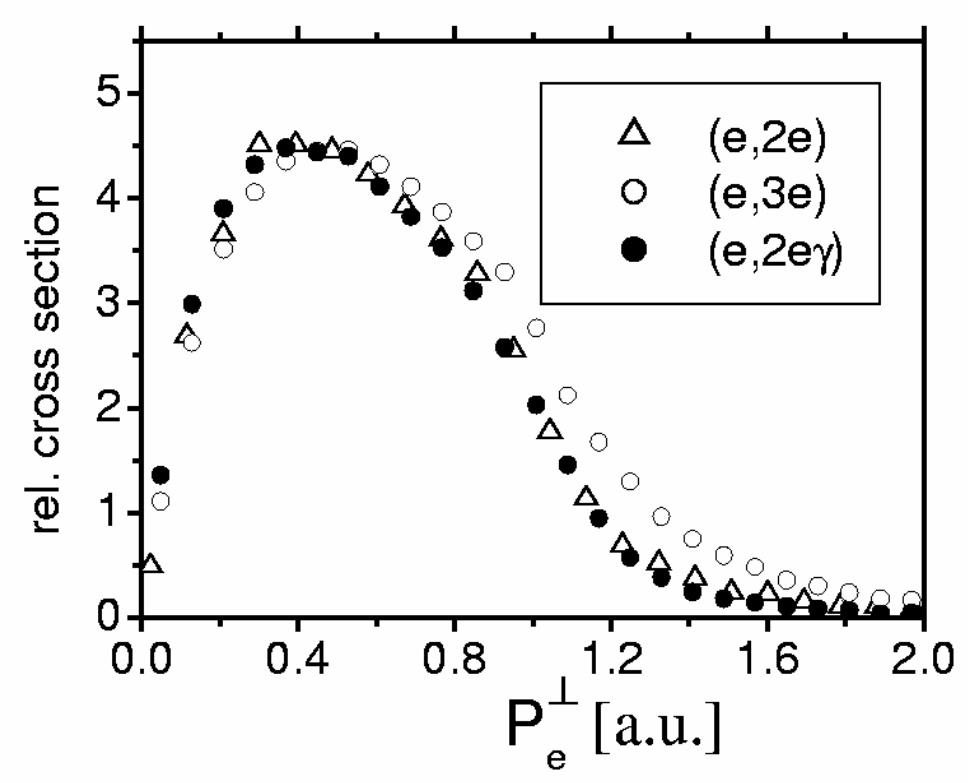

b)

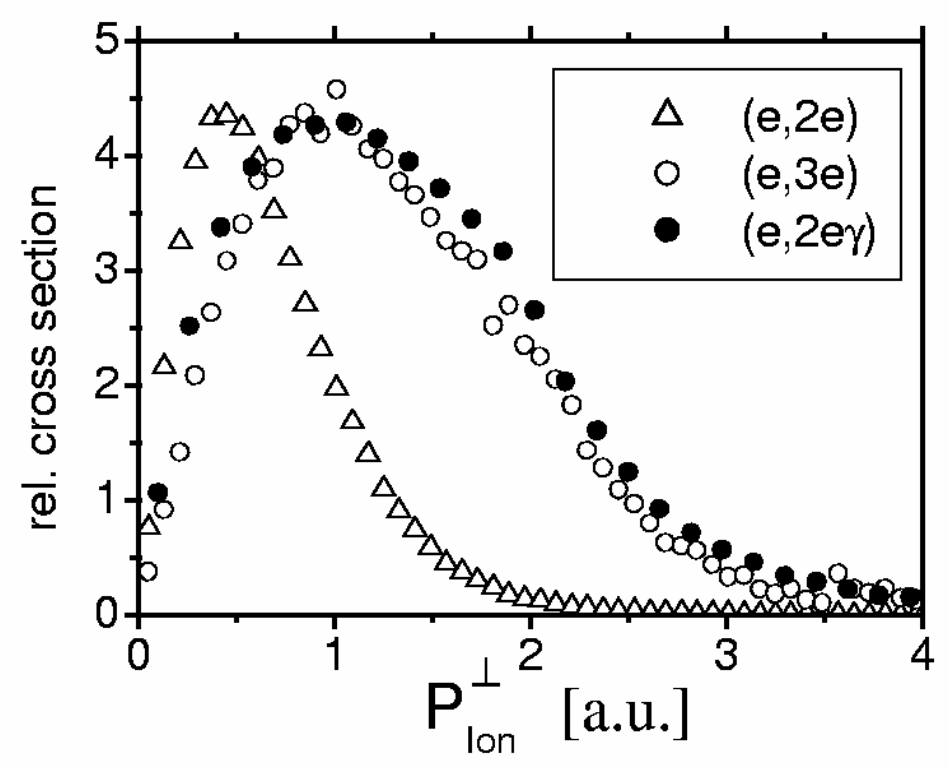




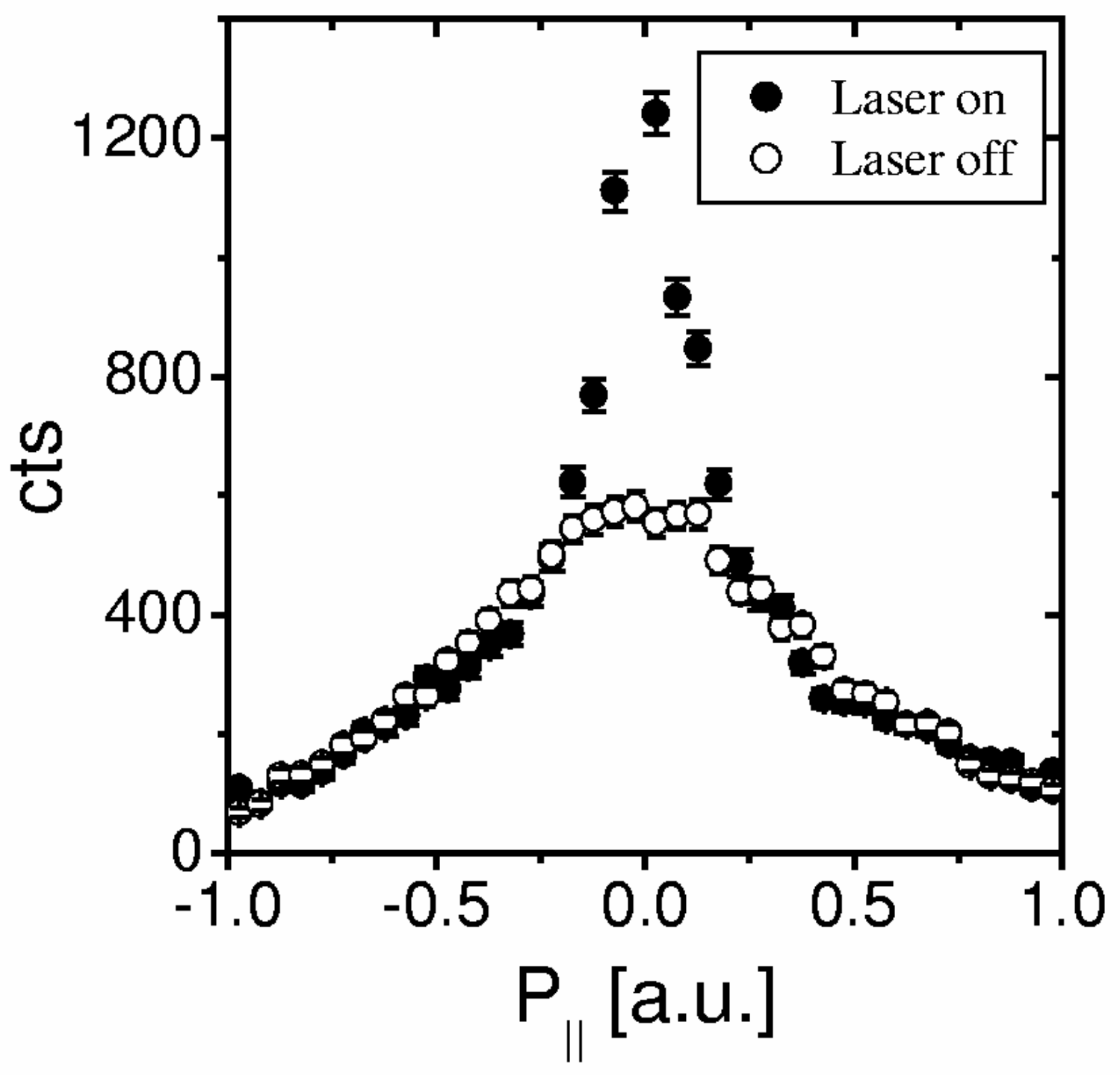

Figure 29 


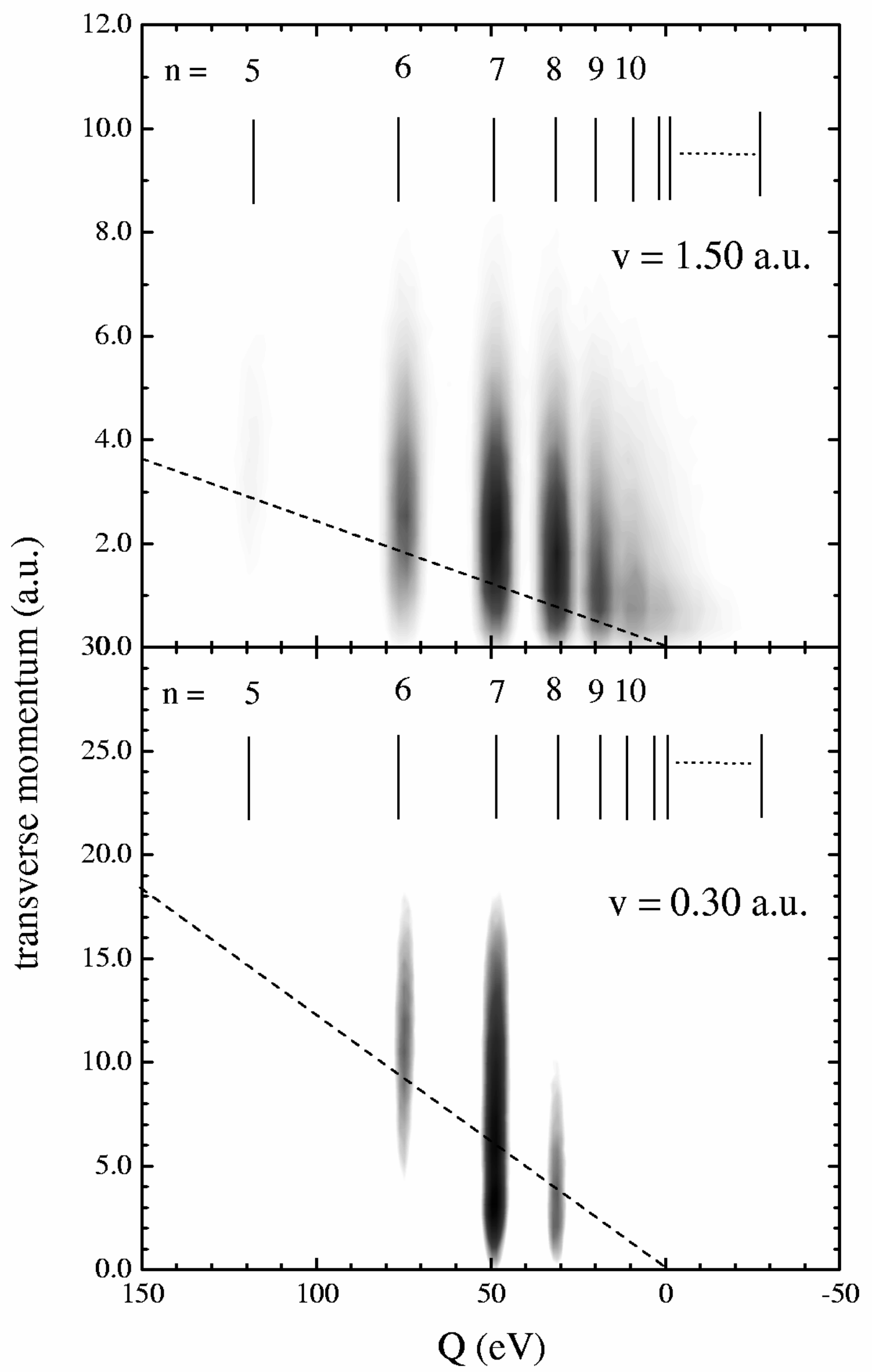

Figure 30 


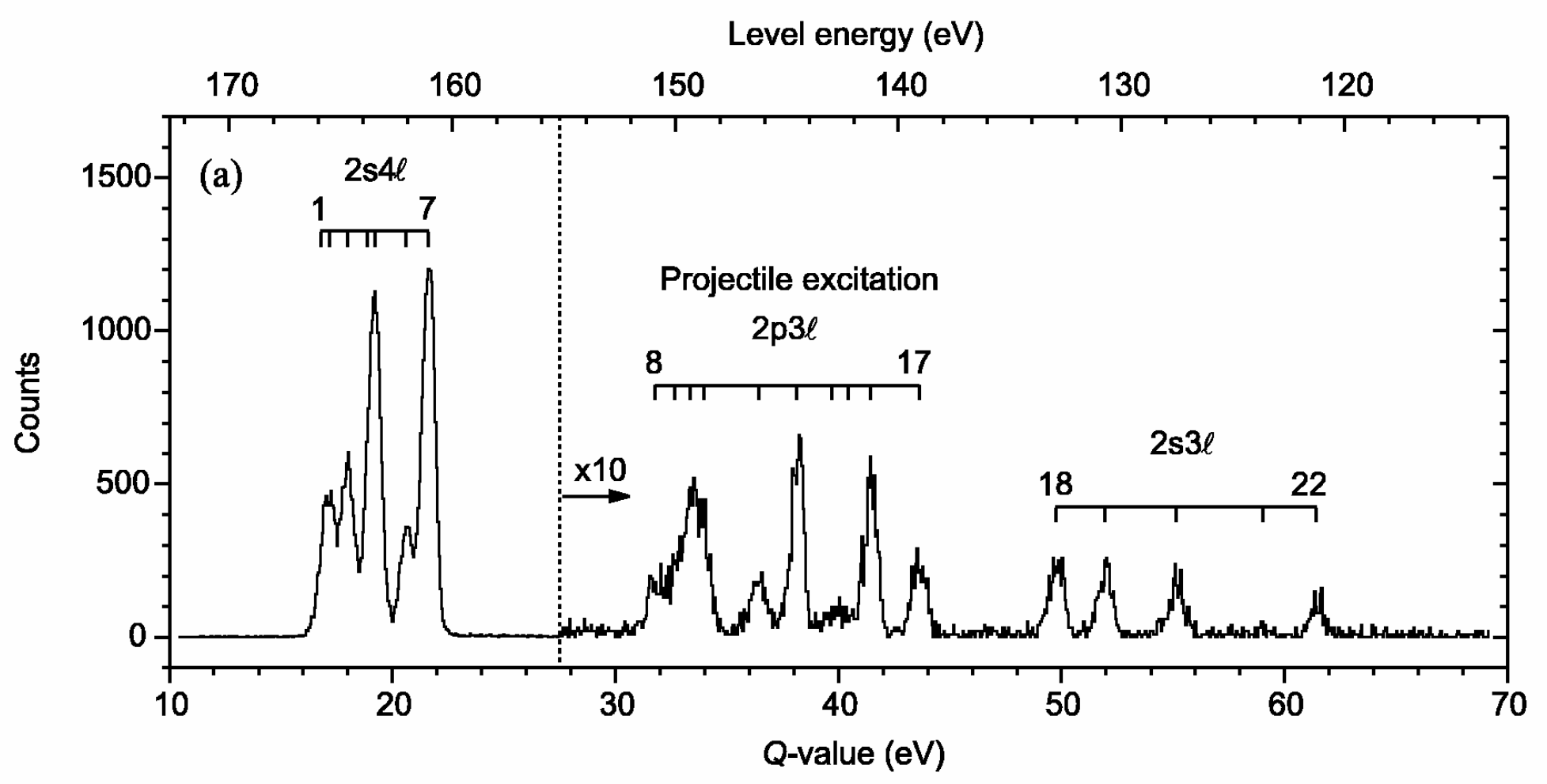

Figure 31 


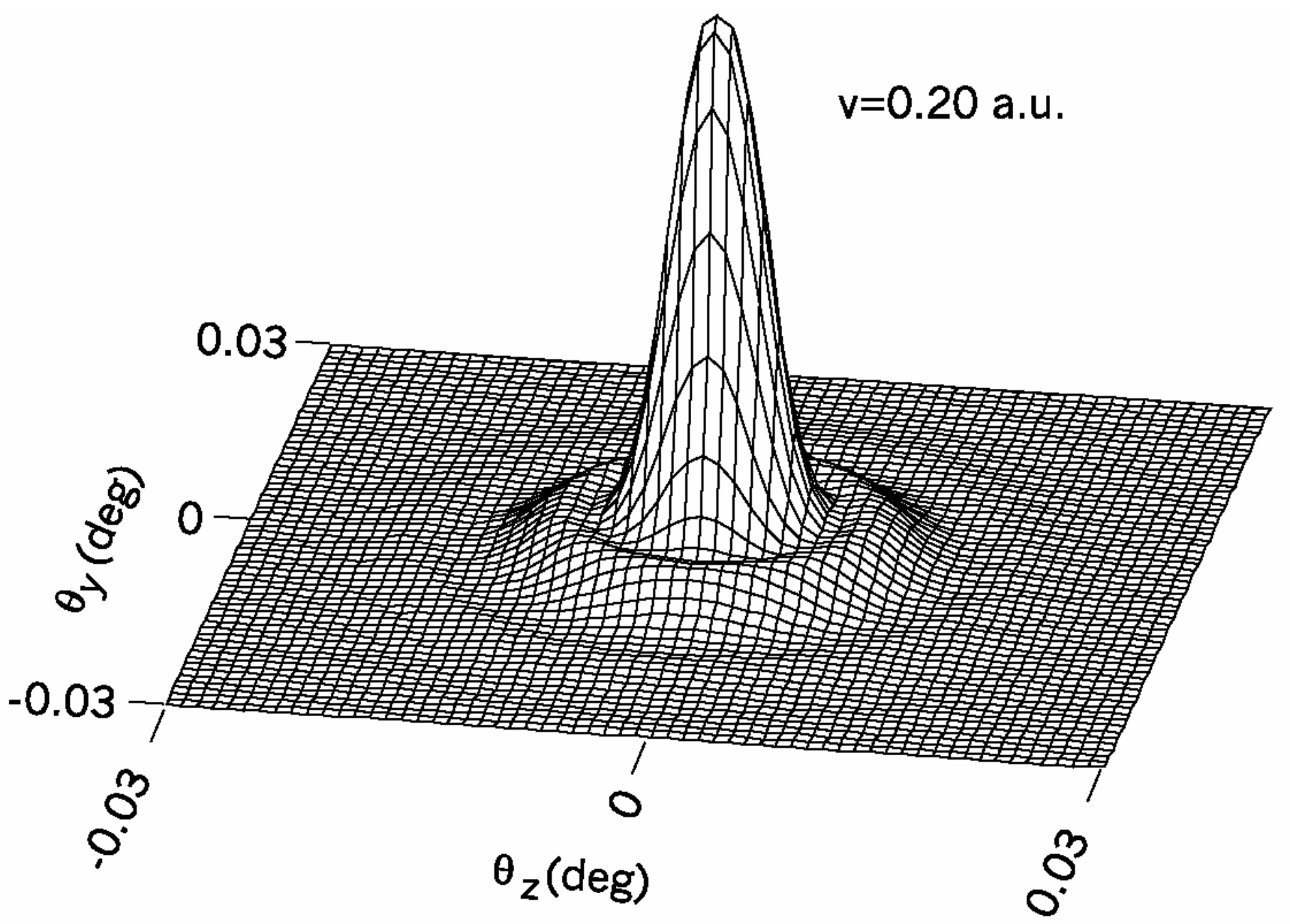

Figure 32 


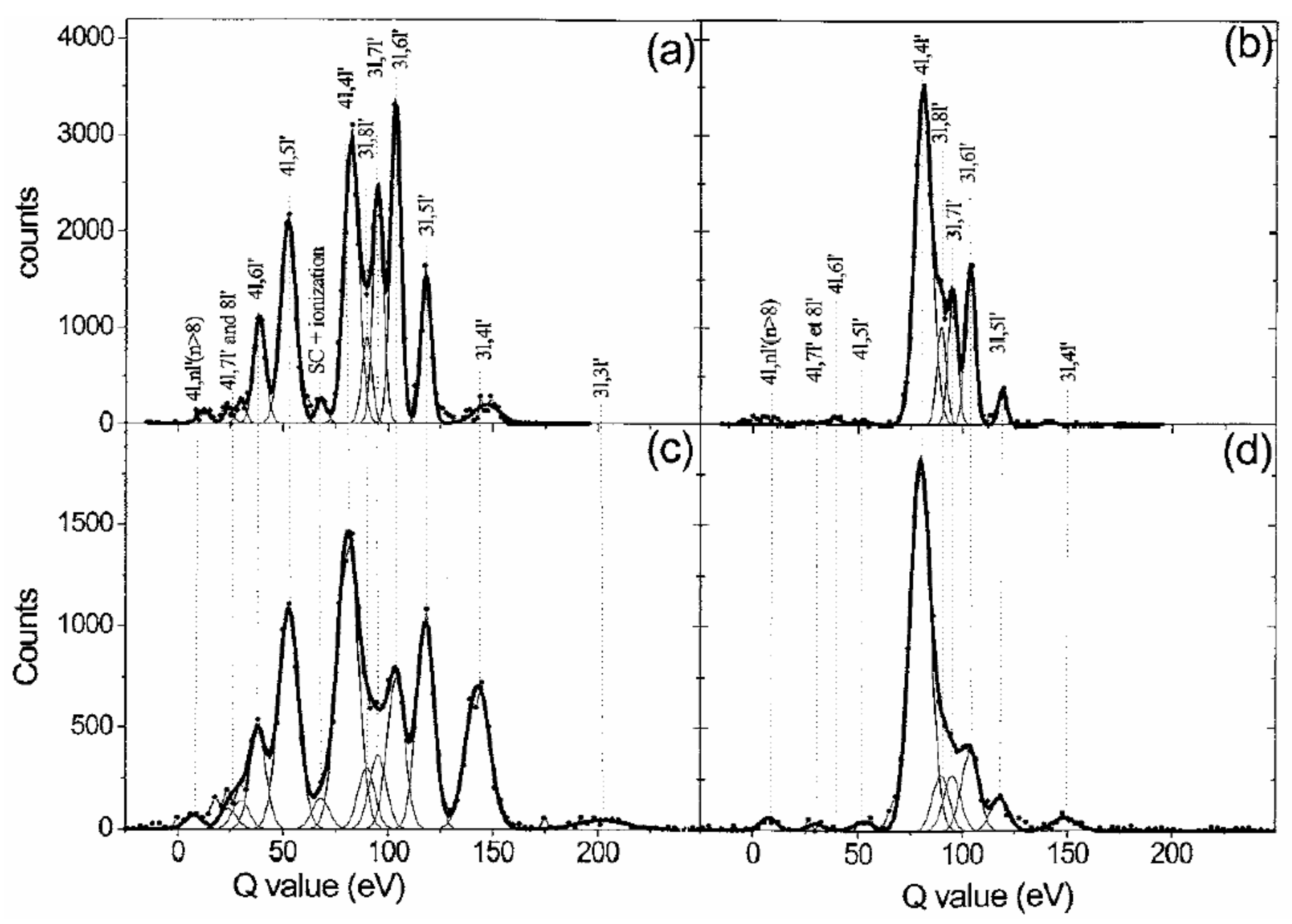

Figure 33 


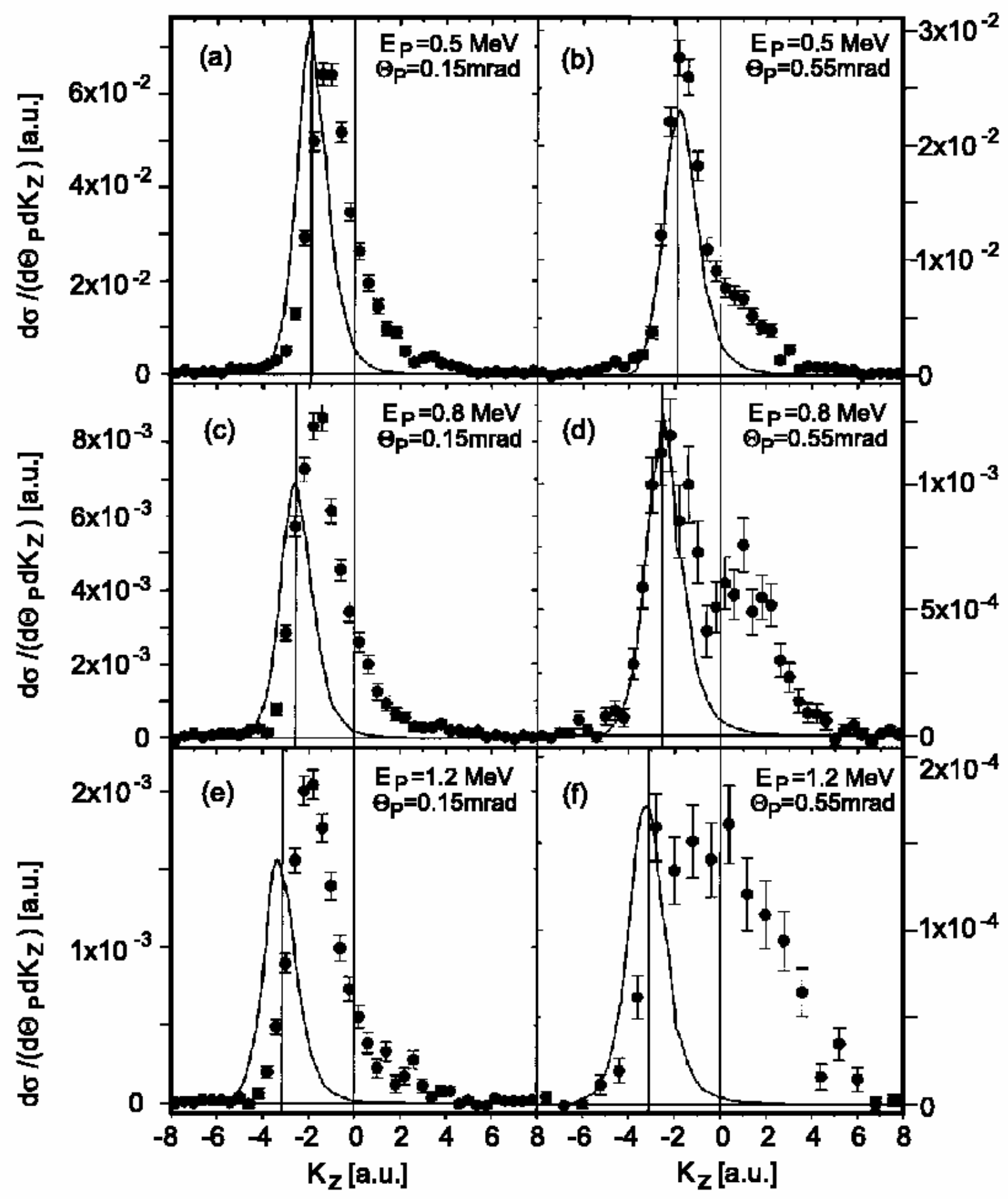

Figure 34 


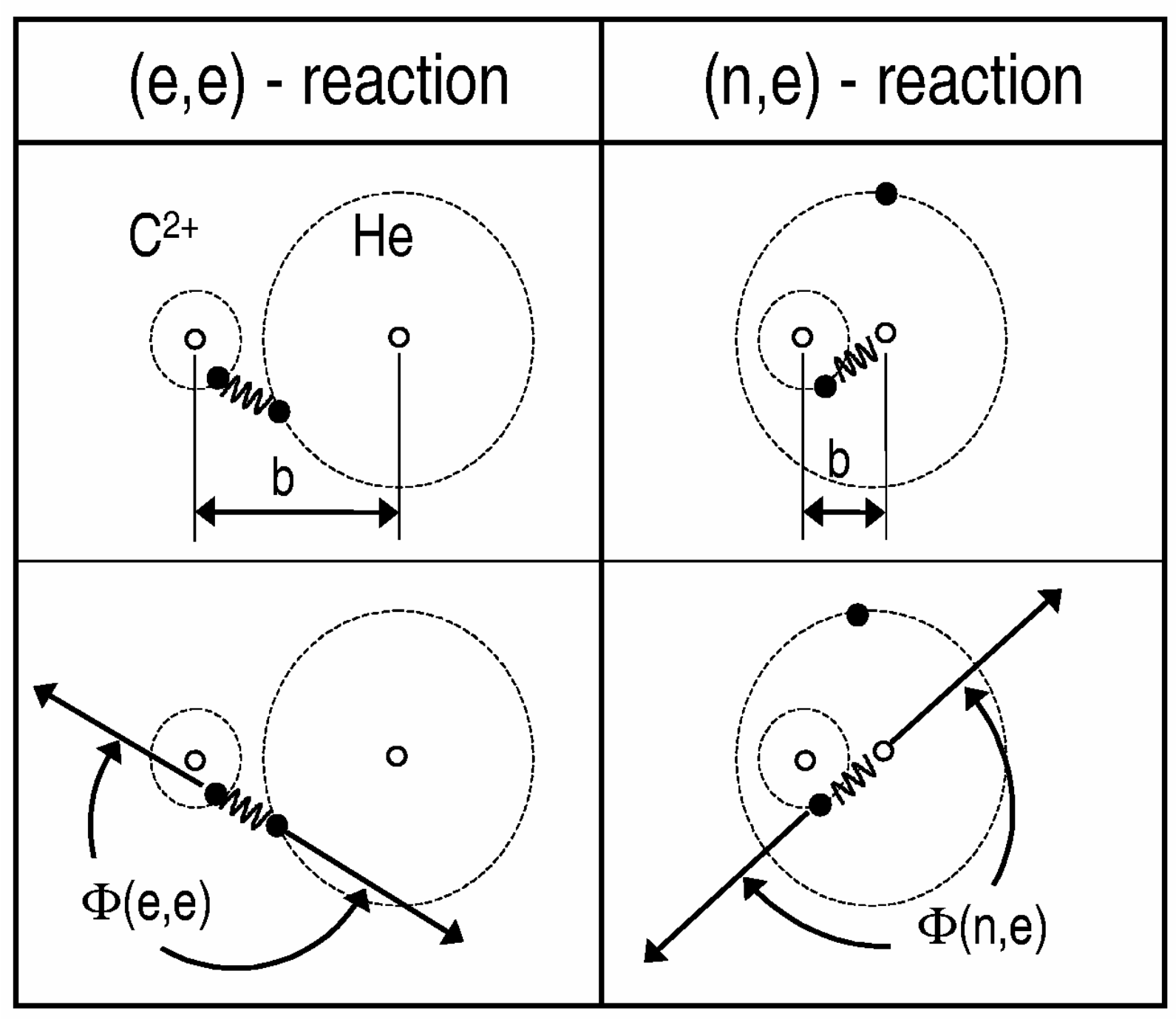

Figure 35 


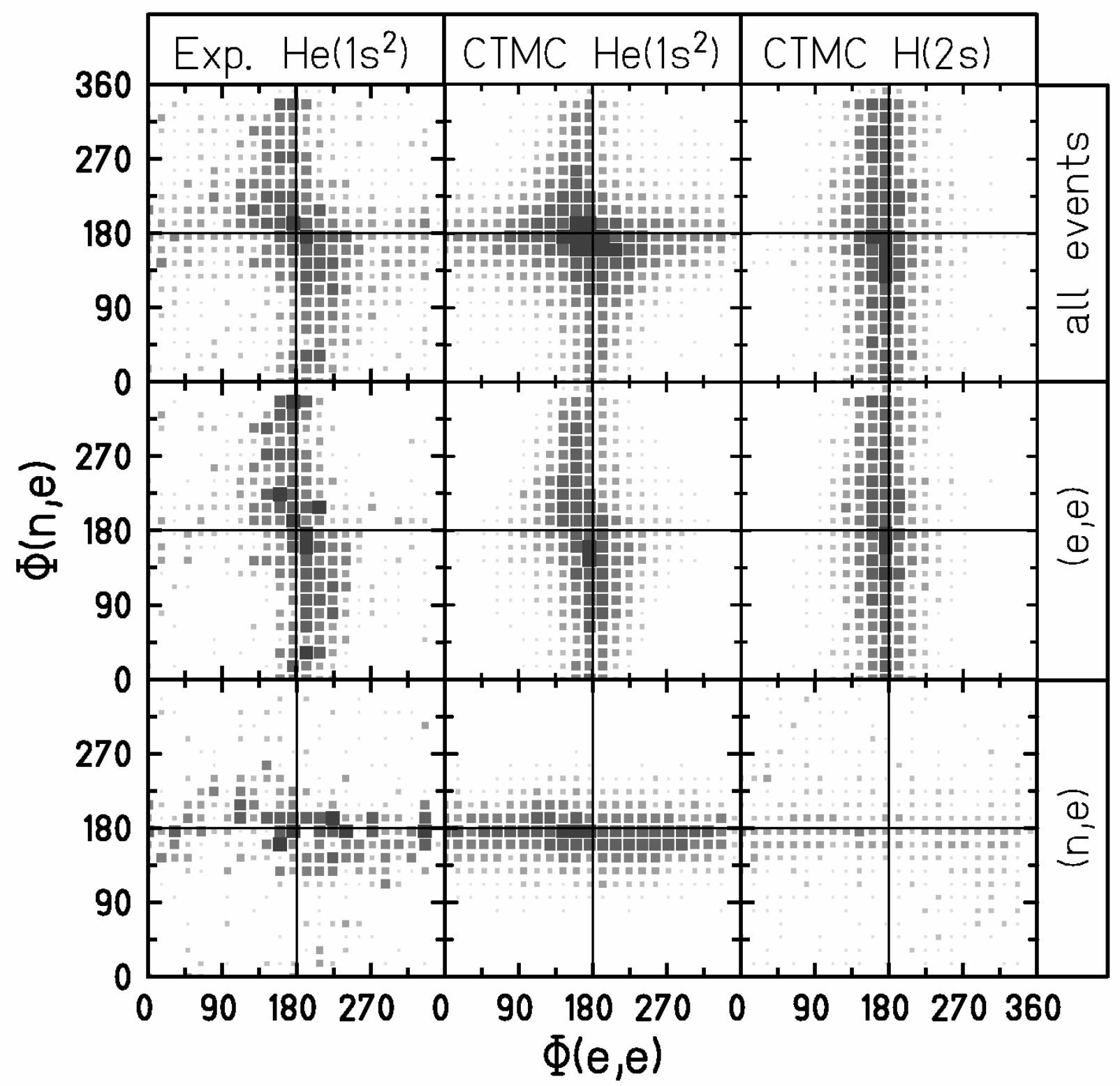

Figure 36 


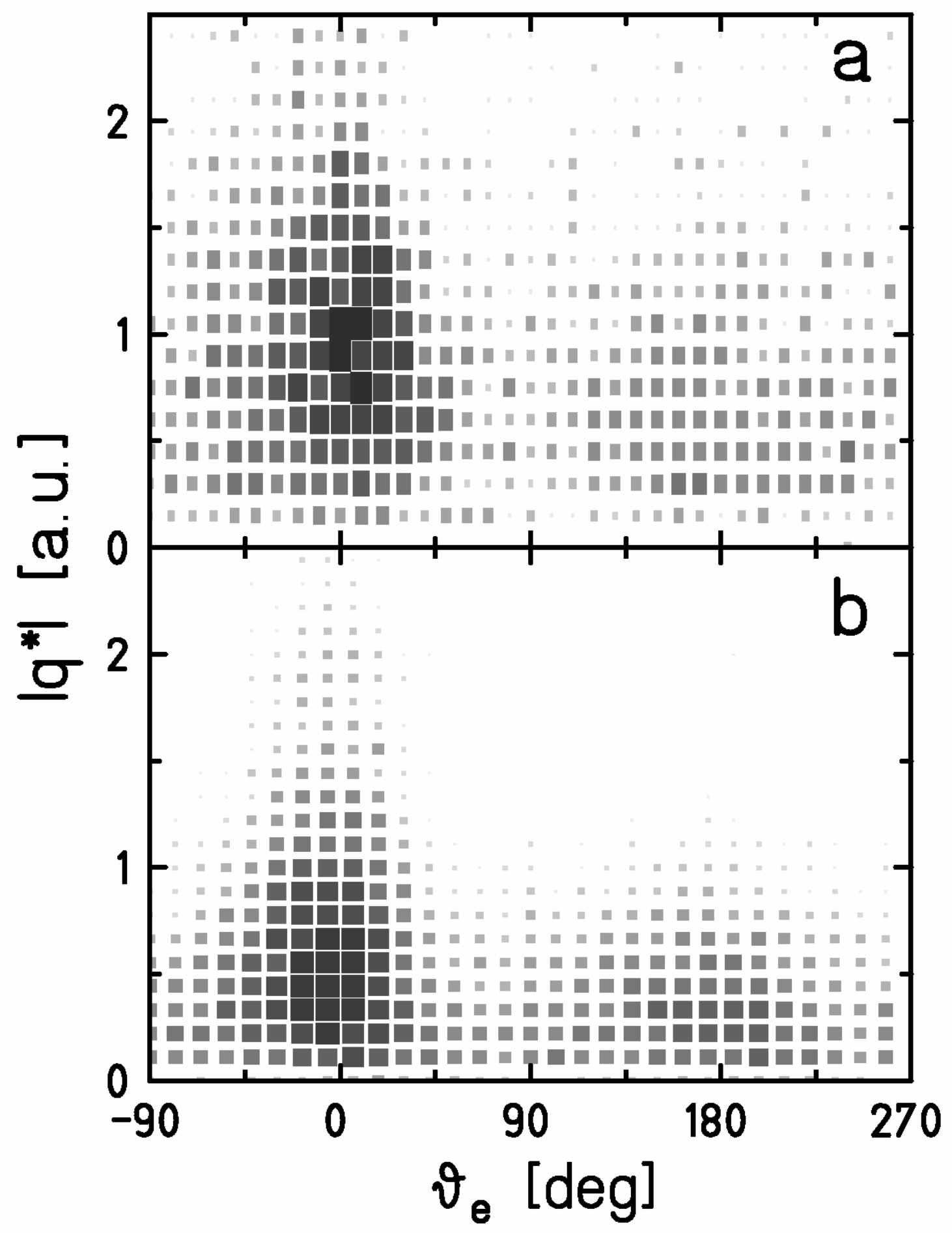

Figure 37 

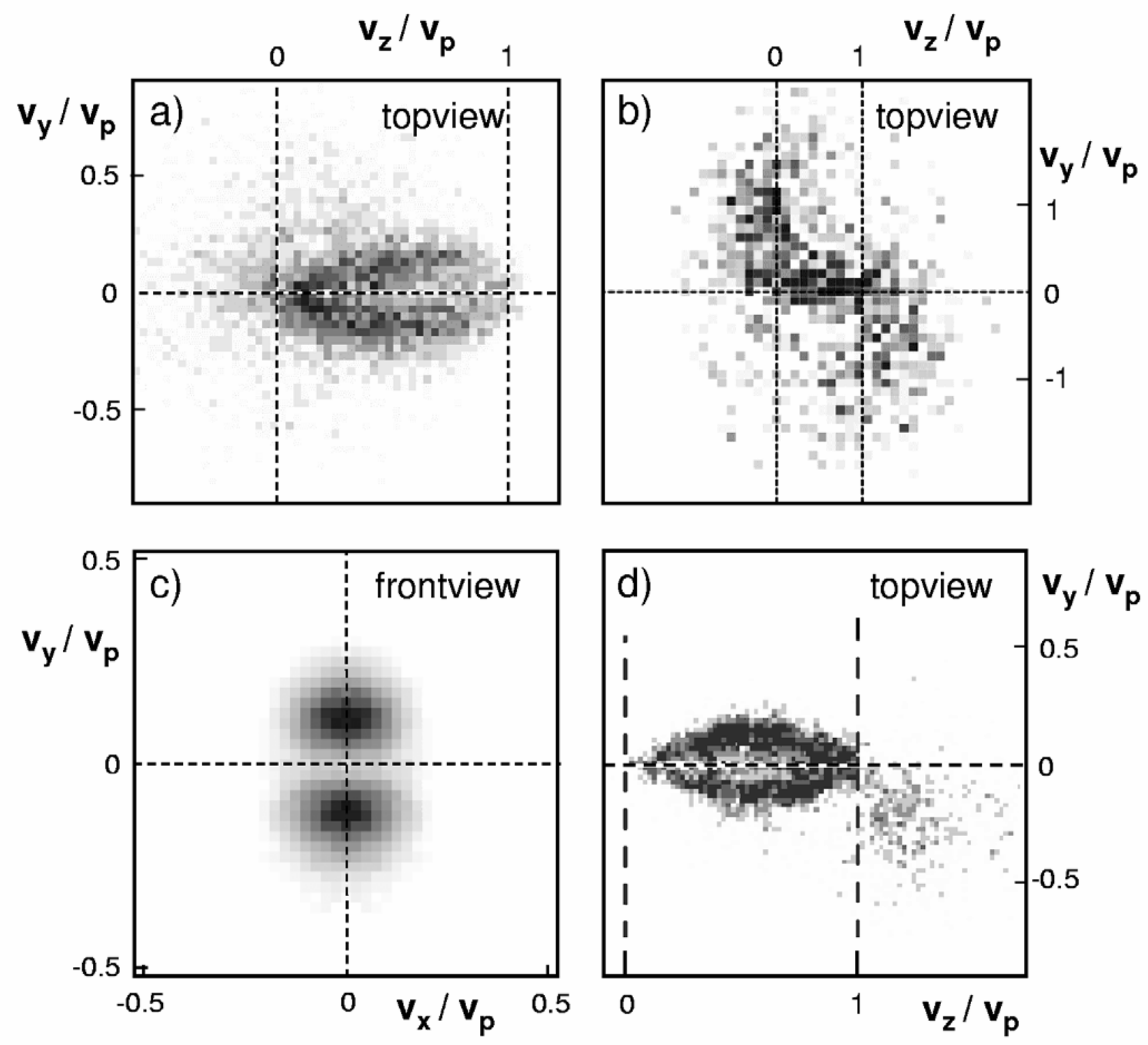

Figure 38 


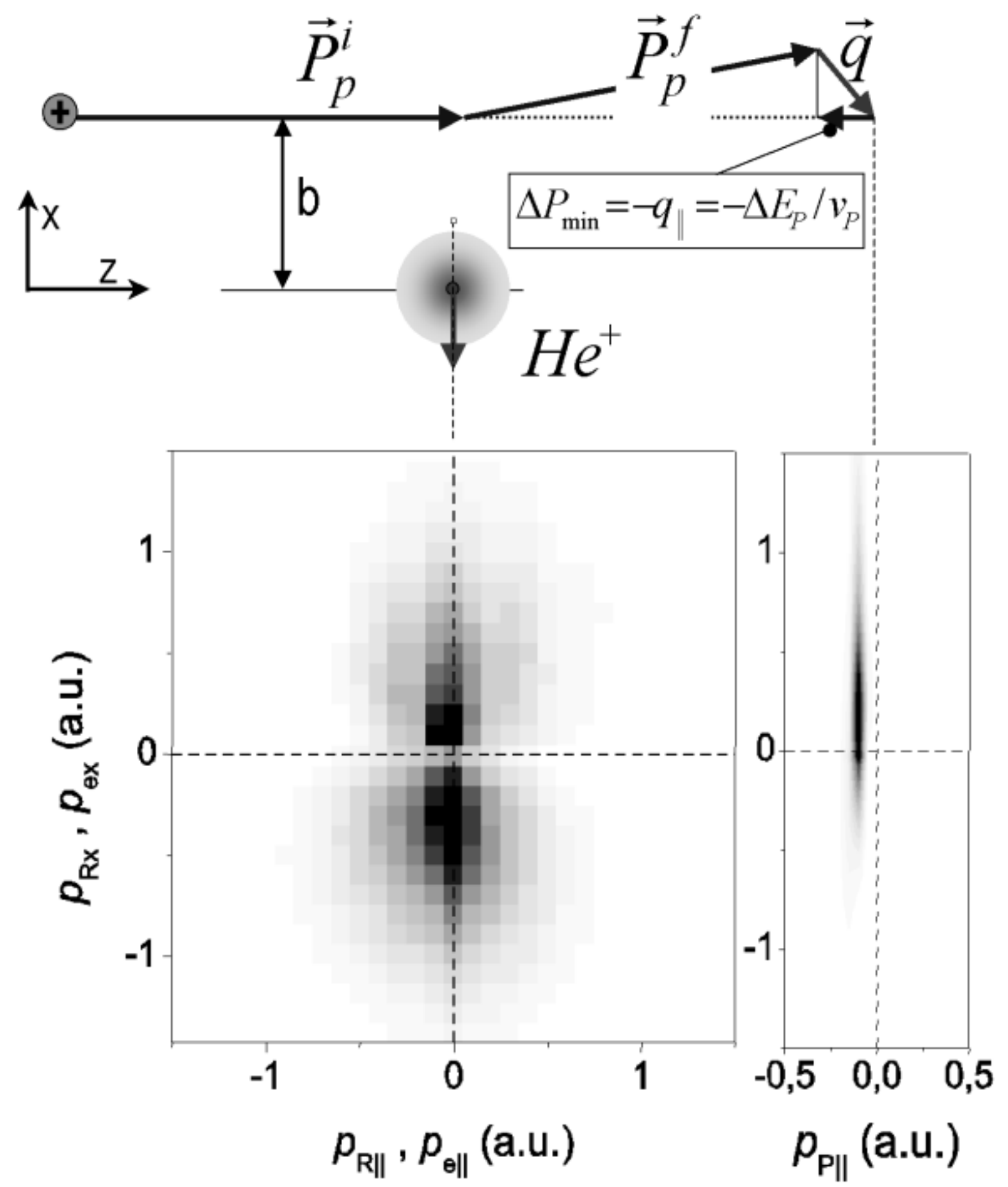

Figure 39 


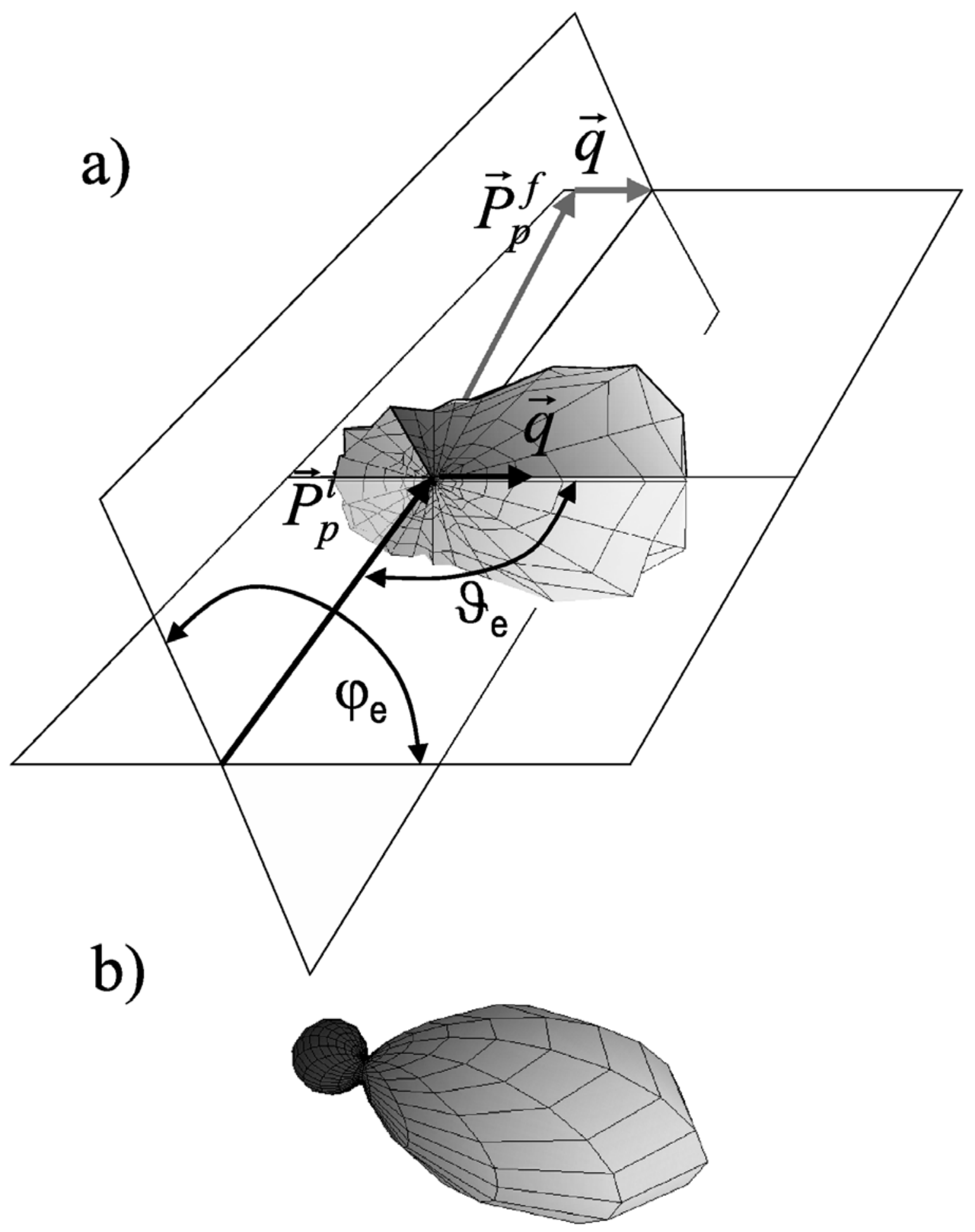

Figure 40 


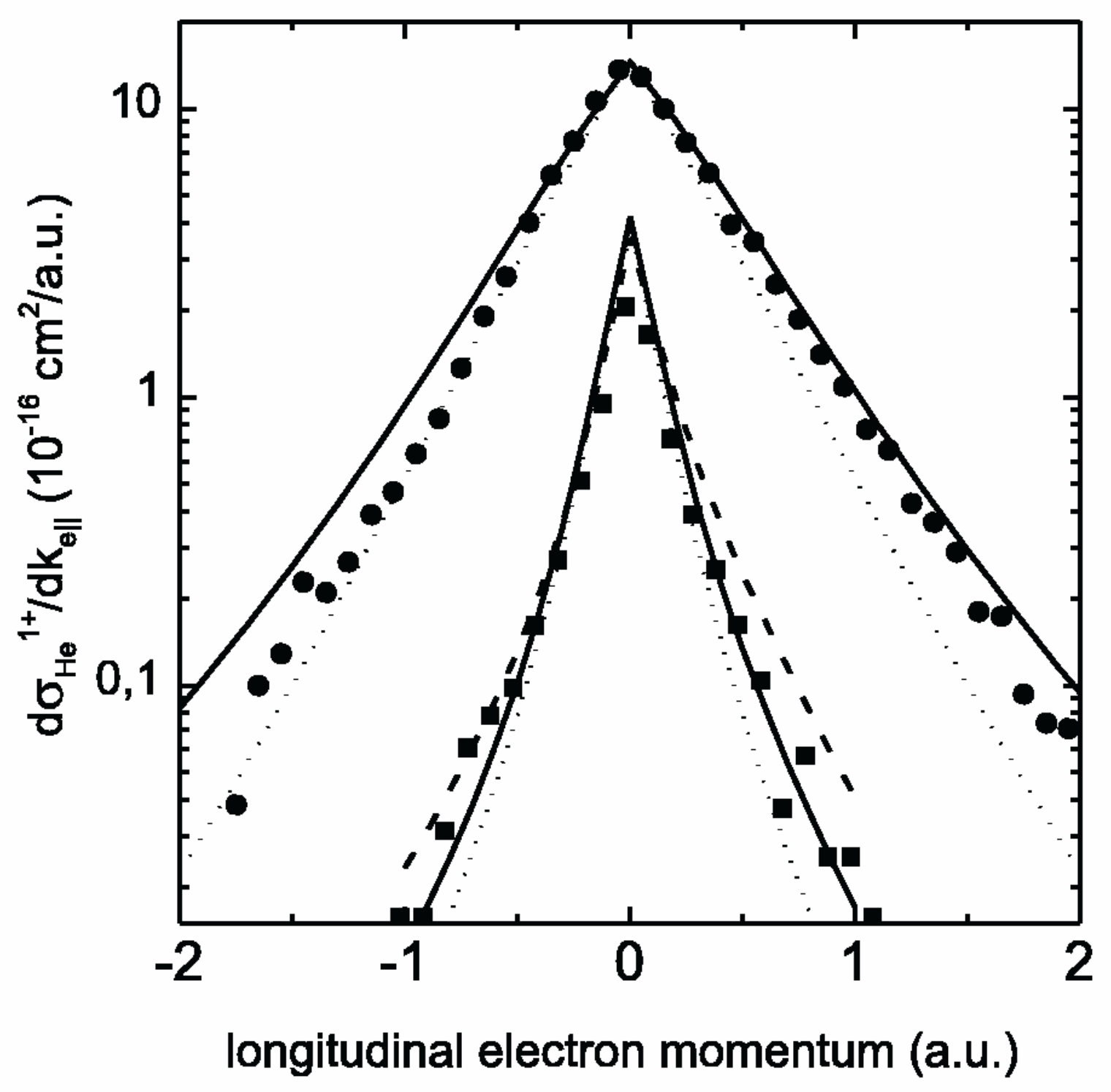

Figure 41 

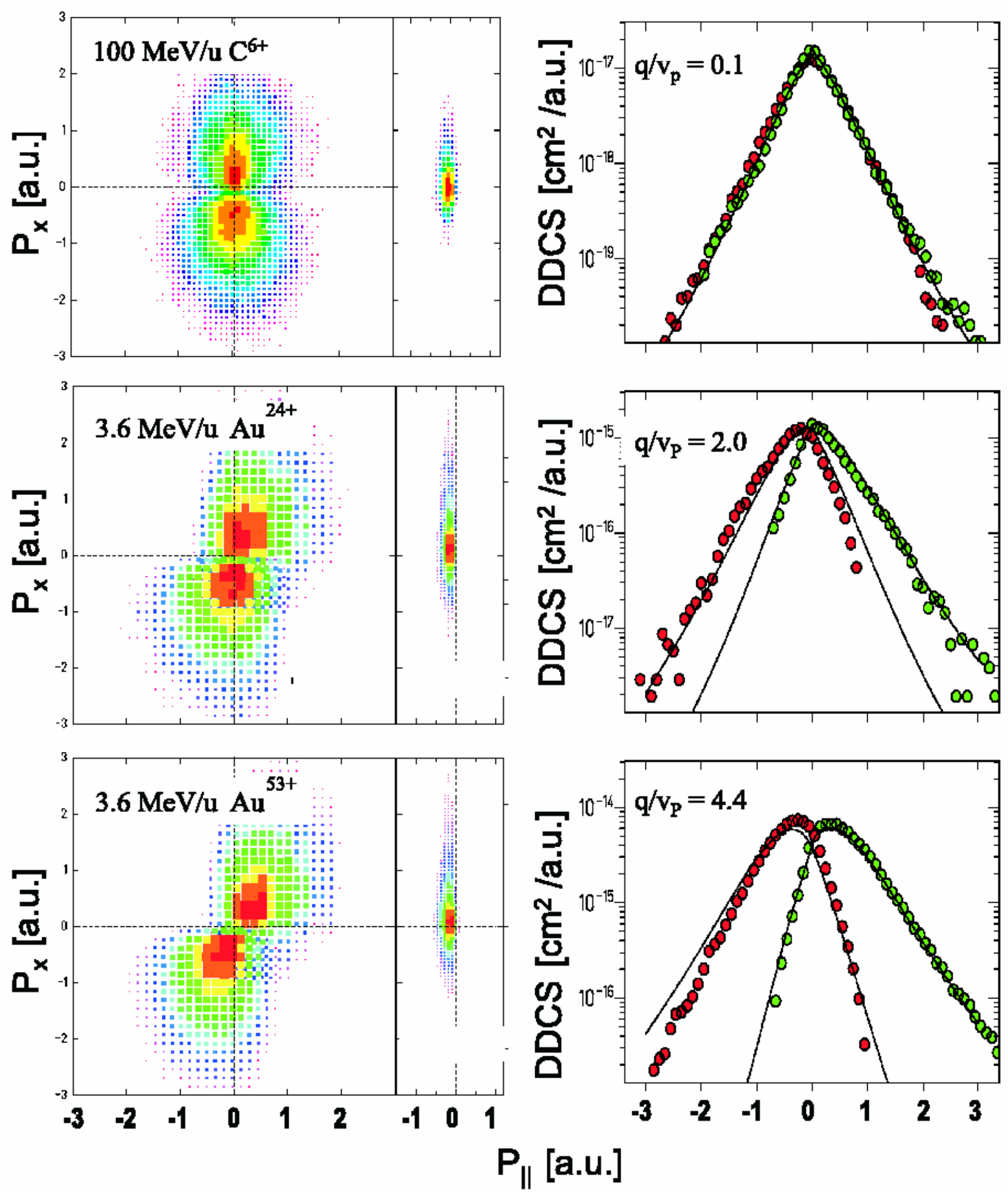

Figure 42 


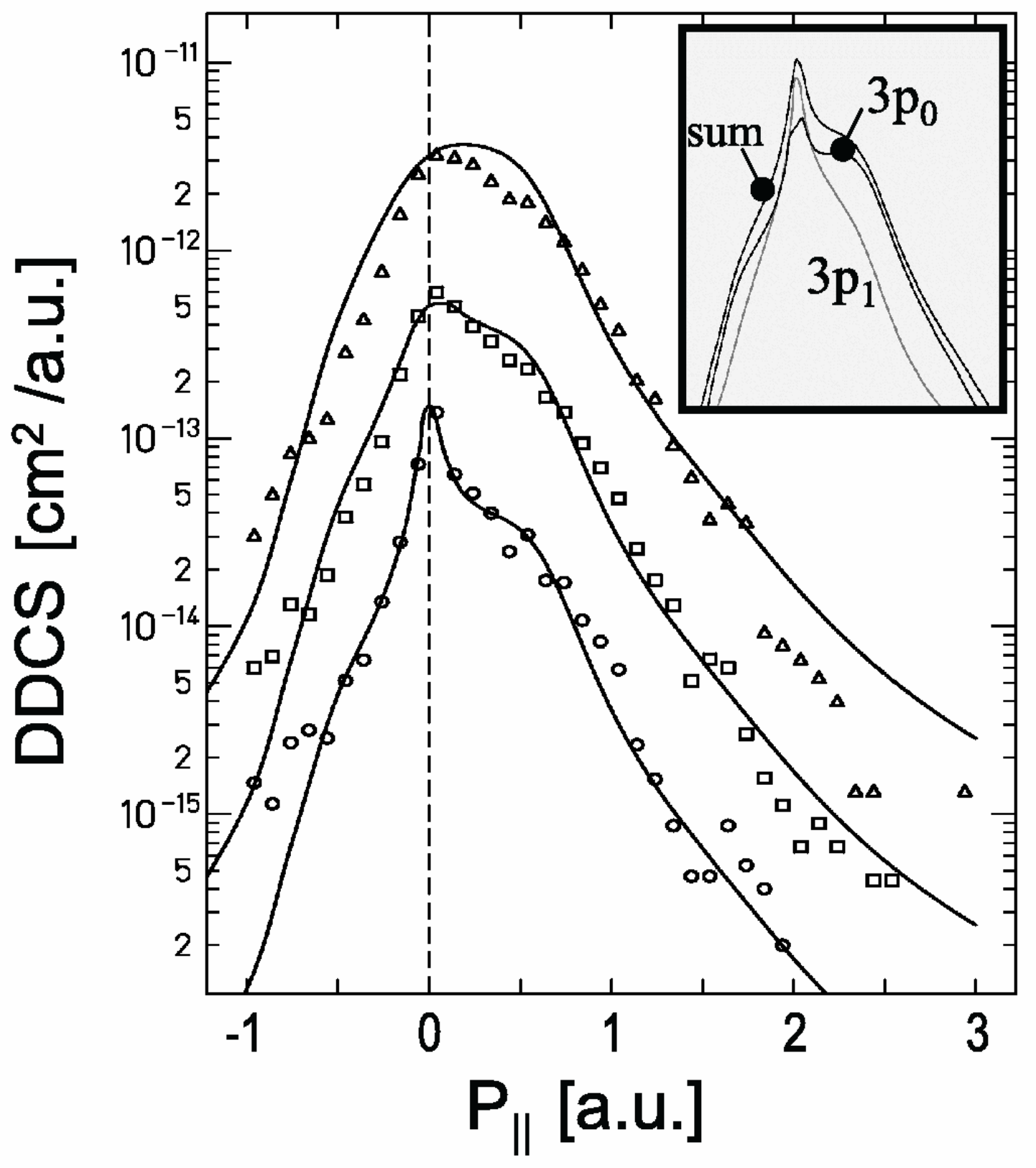

Figure 43 


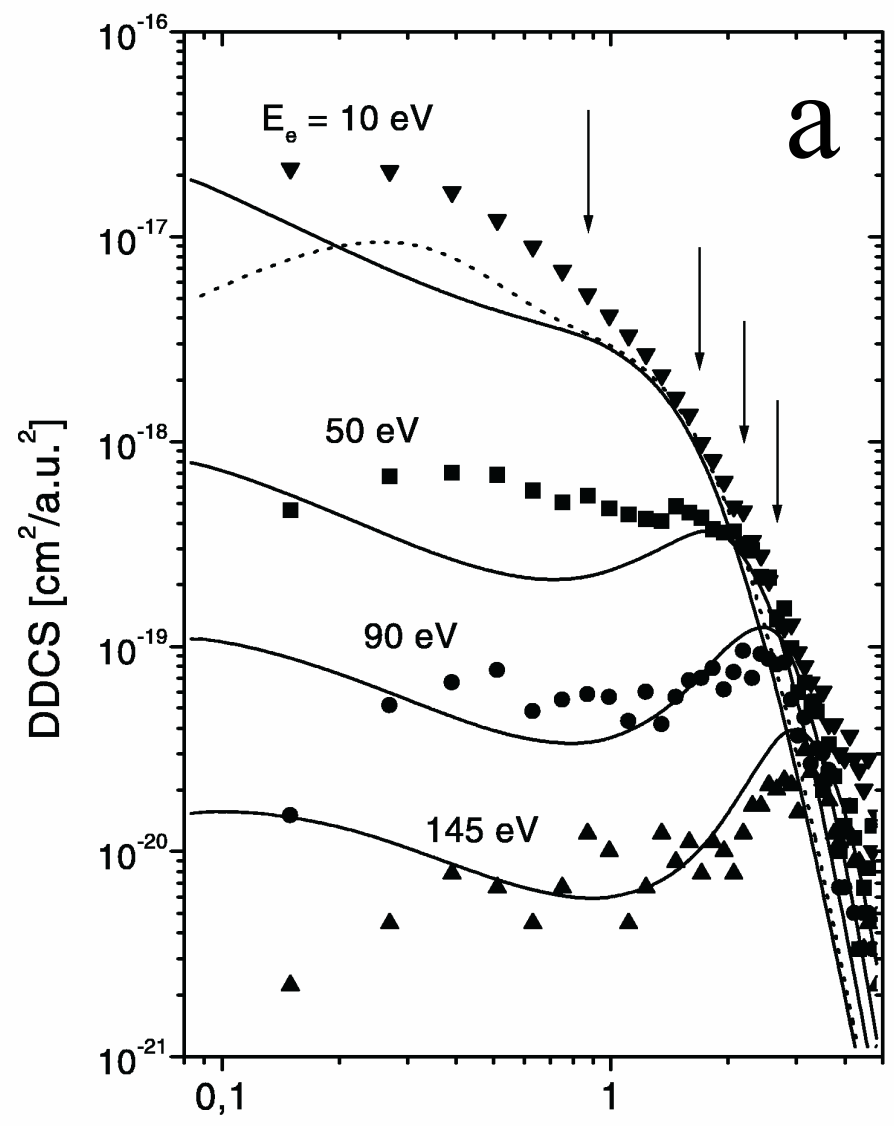

projectile transverse momentum $\mathrm{q}_{\perp}$ [a.u.]

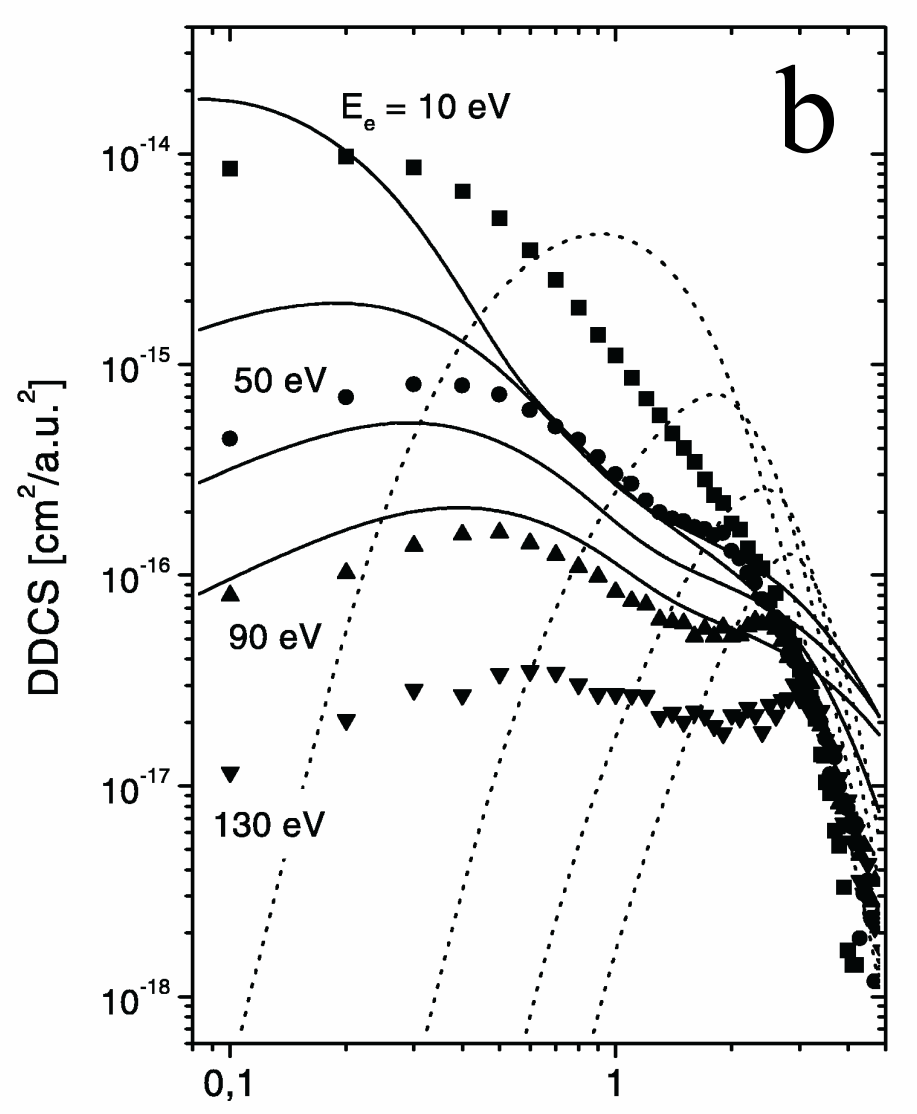

projectile transverse momentum $q_{\perp}$ [a.u.]

Figure 44 

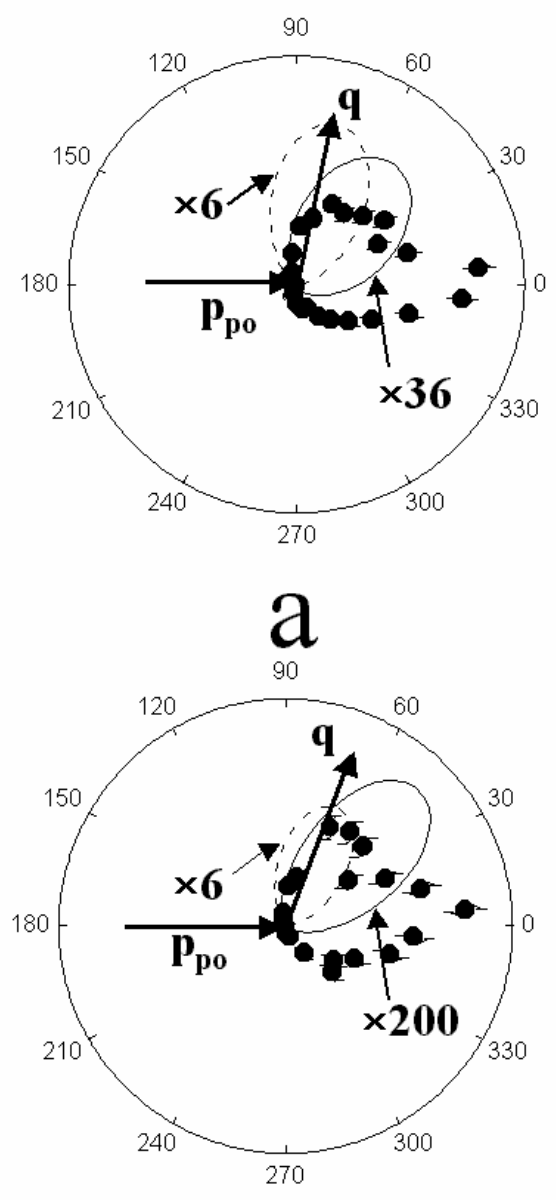
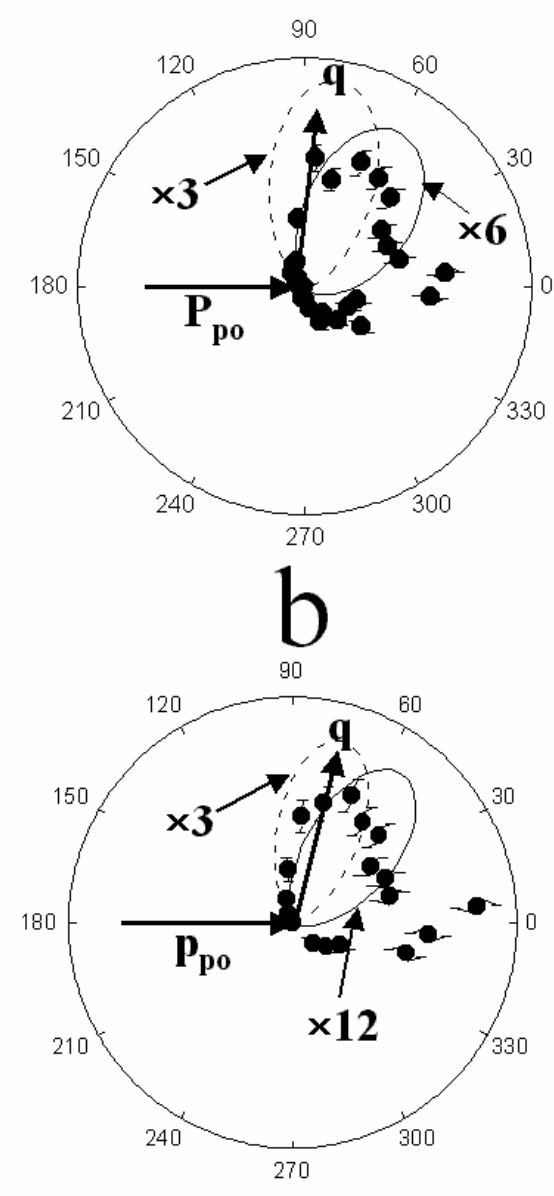

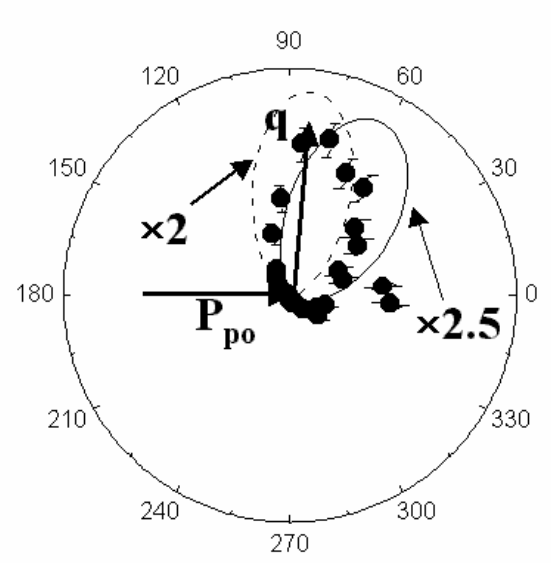

C

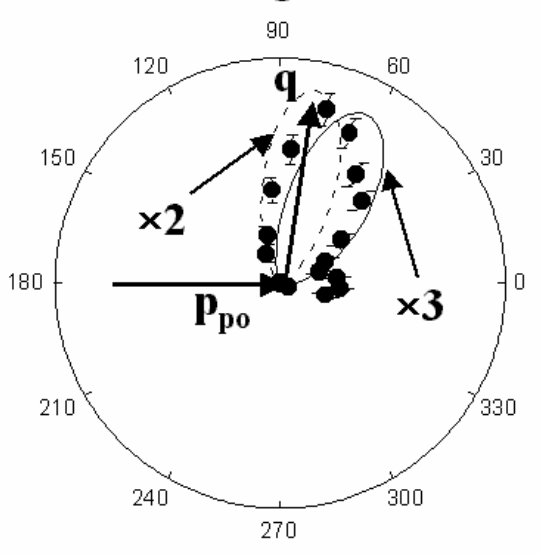

Figure 45 

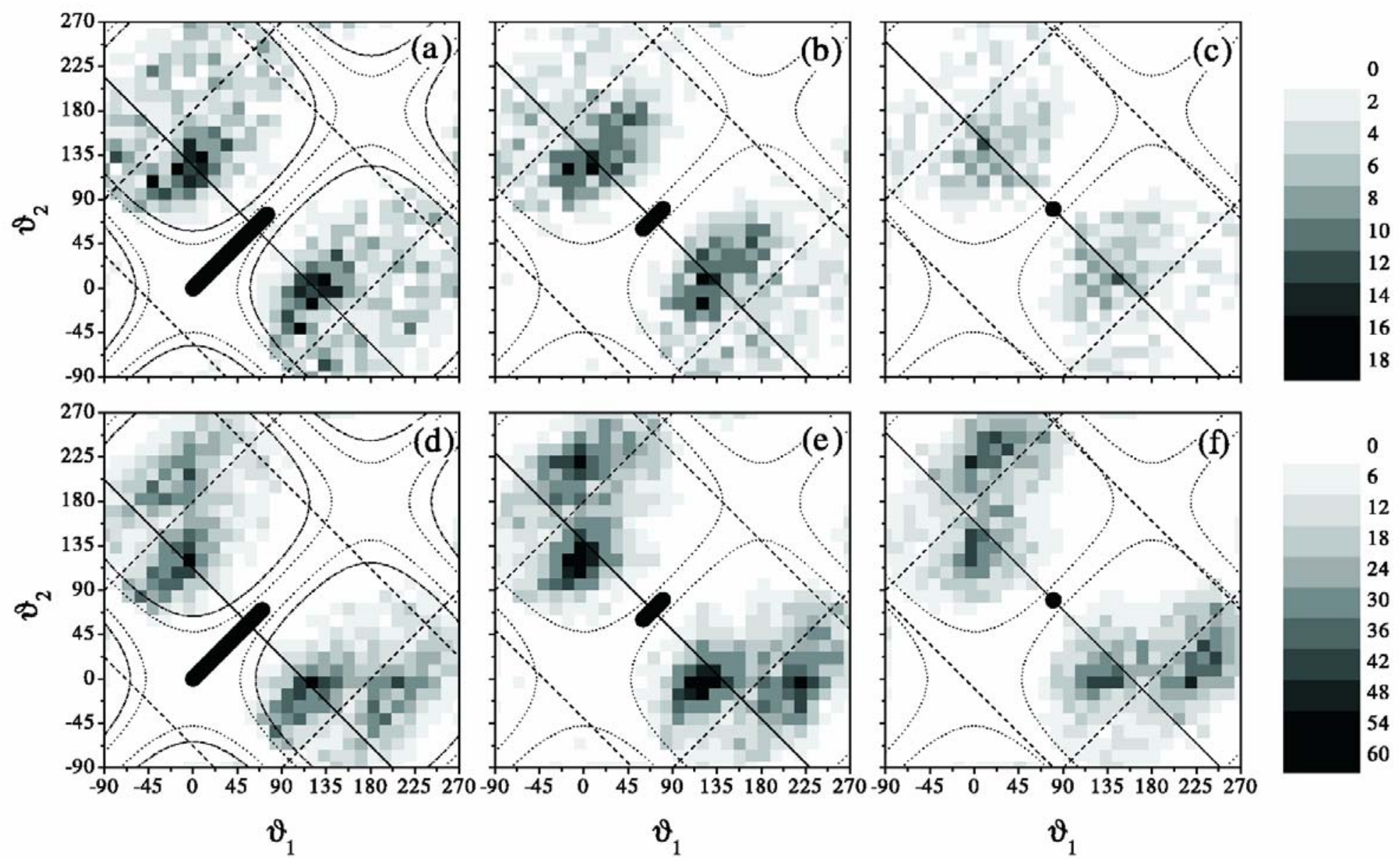

Figure 46 

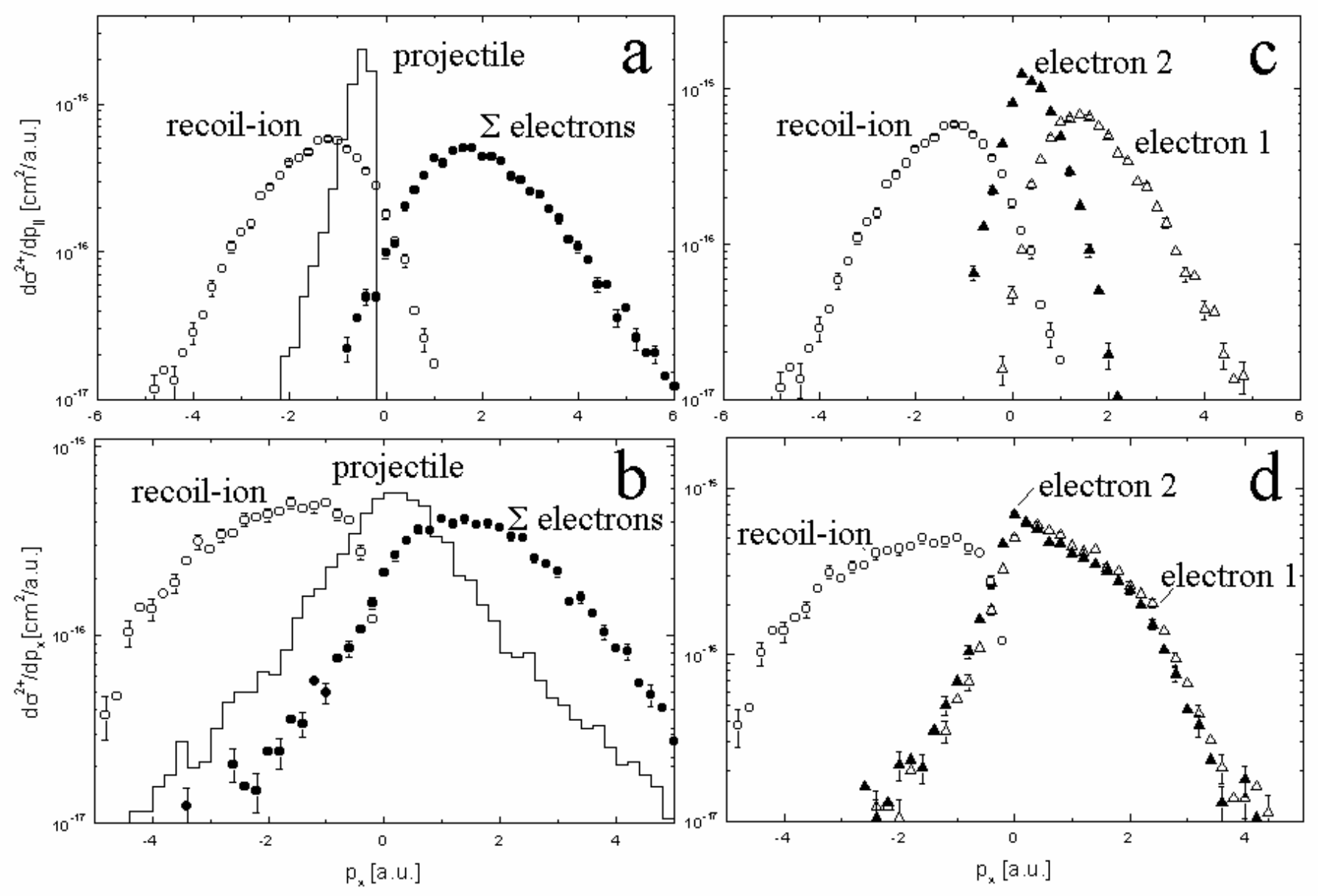

Figure 47 


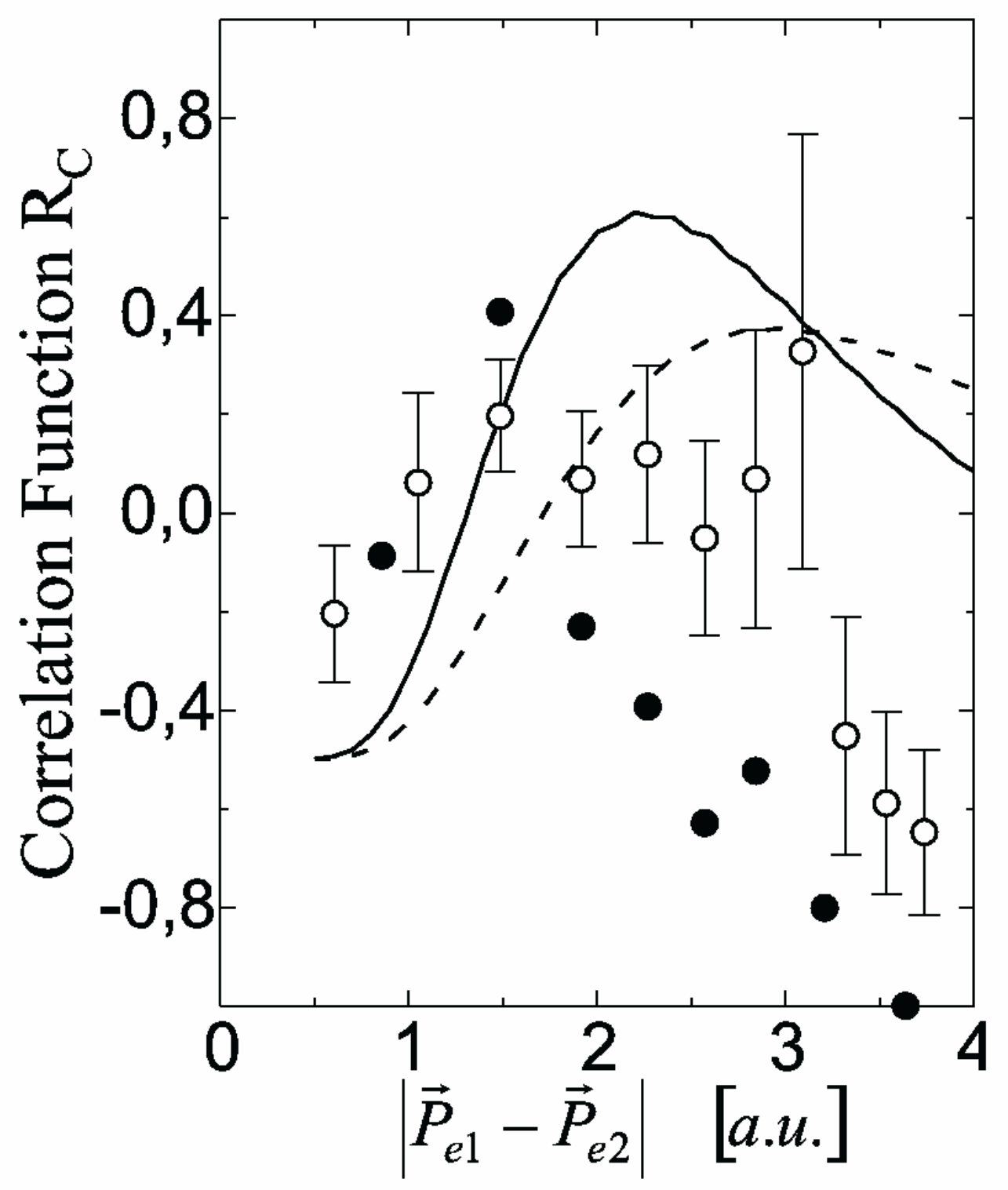

Figure 48 

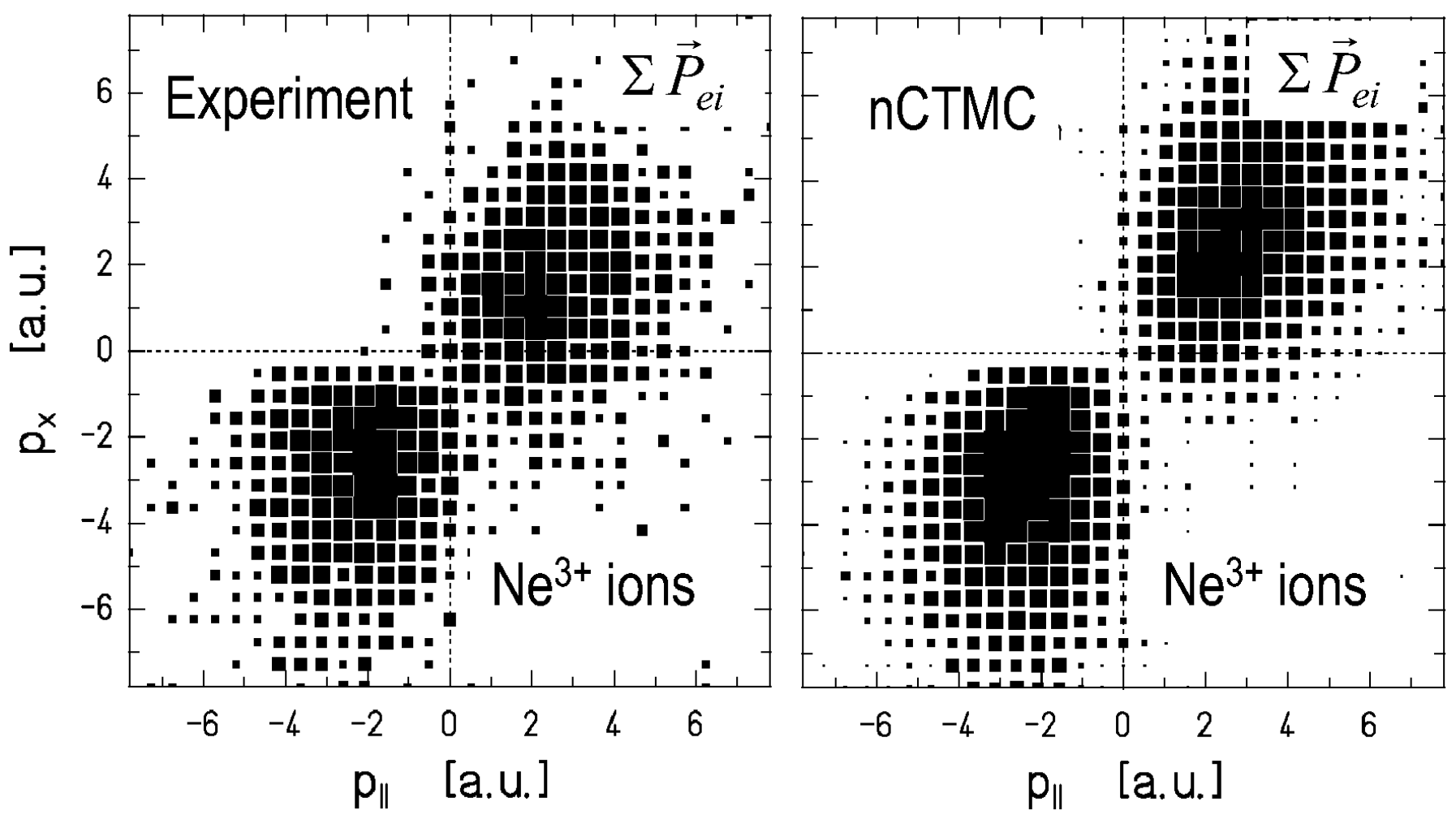

Figure 49 


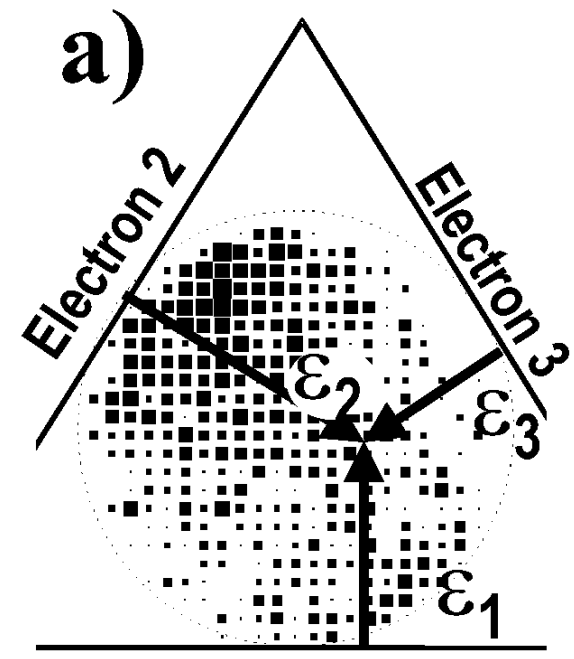

Electron 1
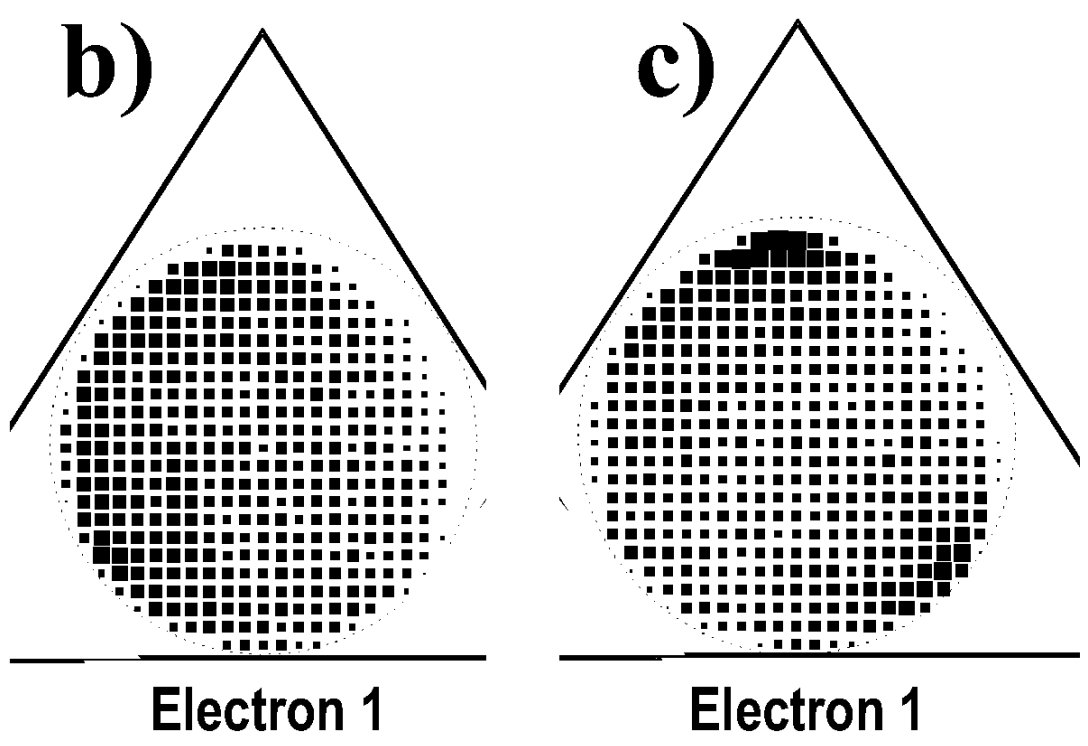

Figure 50 